
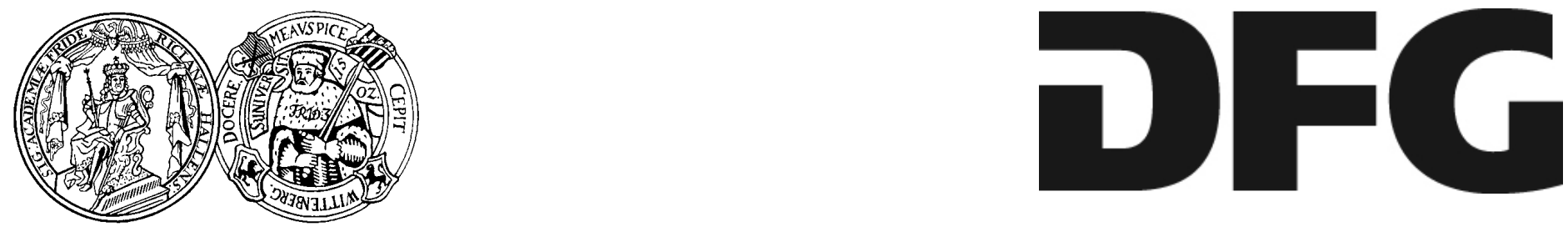

Universitäts- und Landesbibliothek Sachsen-Anhalt

Digitalisierung von Drucken des 18. Jahrhunderts

Ueber den Umgang mit Leidenden

Beneken, Friedrich Burchard

Hannover, 1792

VD18 90745566

urn:nbn:de:gbv:3:1-840212 
Foos

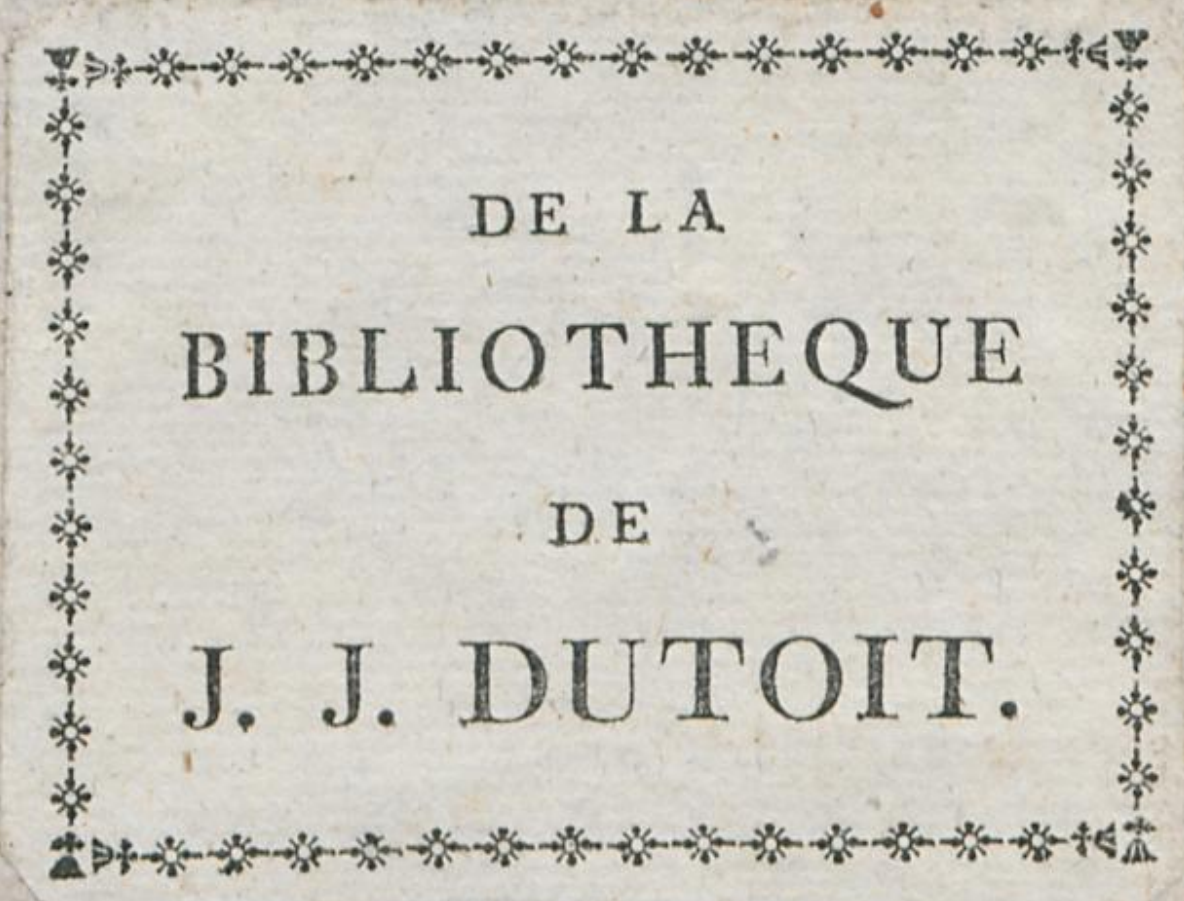




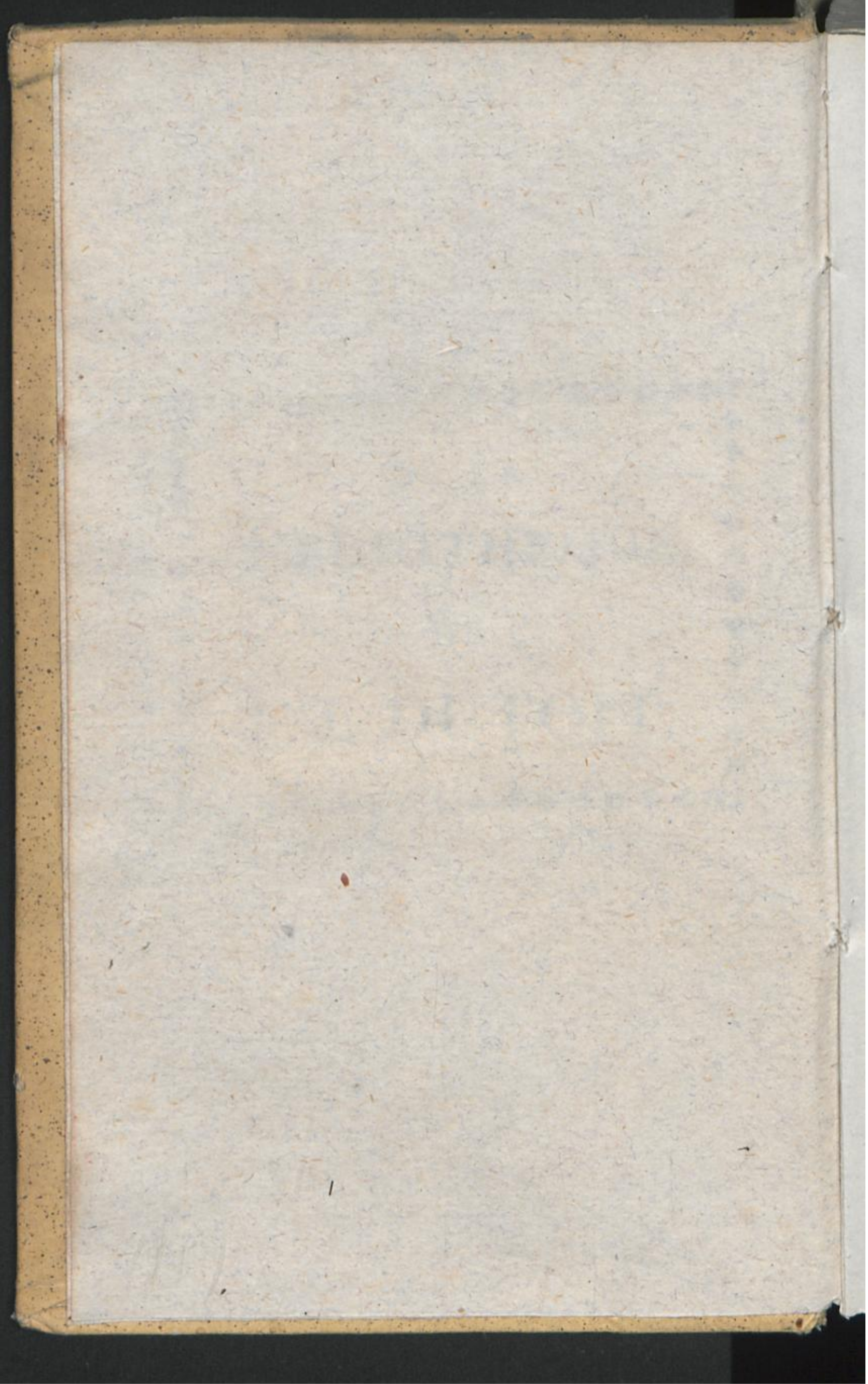




\title{
$\mathfrak{H} e b e \mathfrak{F}$
}

Dett

\section{Ututgants mit Selbettoent.}

\author{
Şetausgegebea
}

Do it

Srieorid) פurdiaro Beneten, Paffor Diac. in Romnenbers.

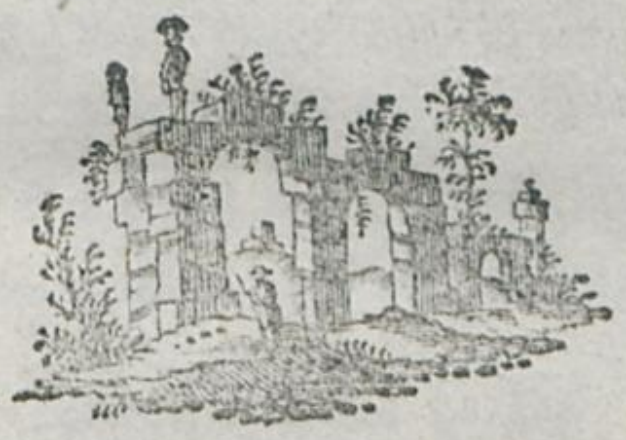

Erfter Iheil.

So $n\|\| D$ D $~ r$,

bey (5)riftian aitfder,

I 792 . 

Shro Sadtyuruben Sinaben

ber গ⿺e

Smalie Frtederife von 230 enfers:

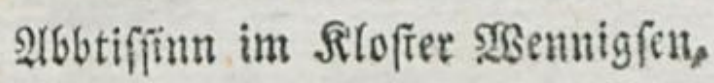
un $\mathrm{O}$

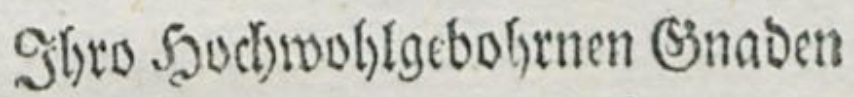

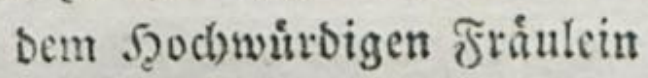
gricoctikgonll fiat,

Eonventualim im Slofter $2 B$ ennigfen,

elyerbietigit und Danflor

gewionet. 


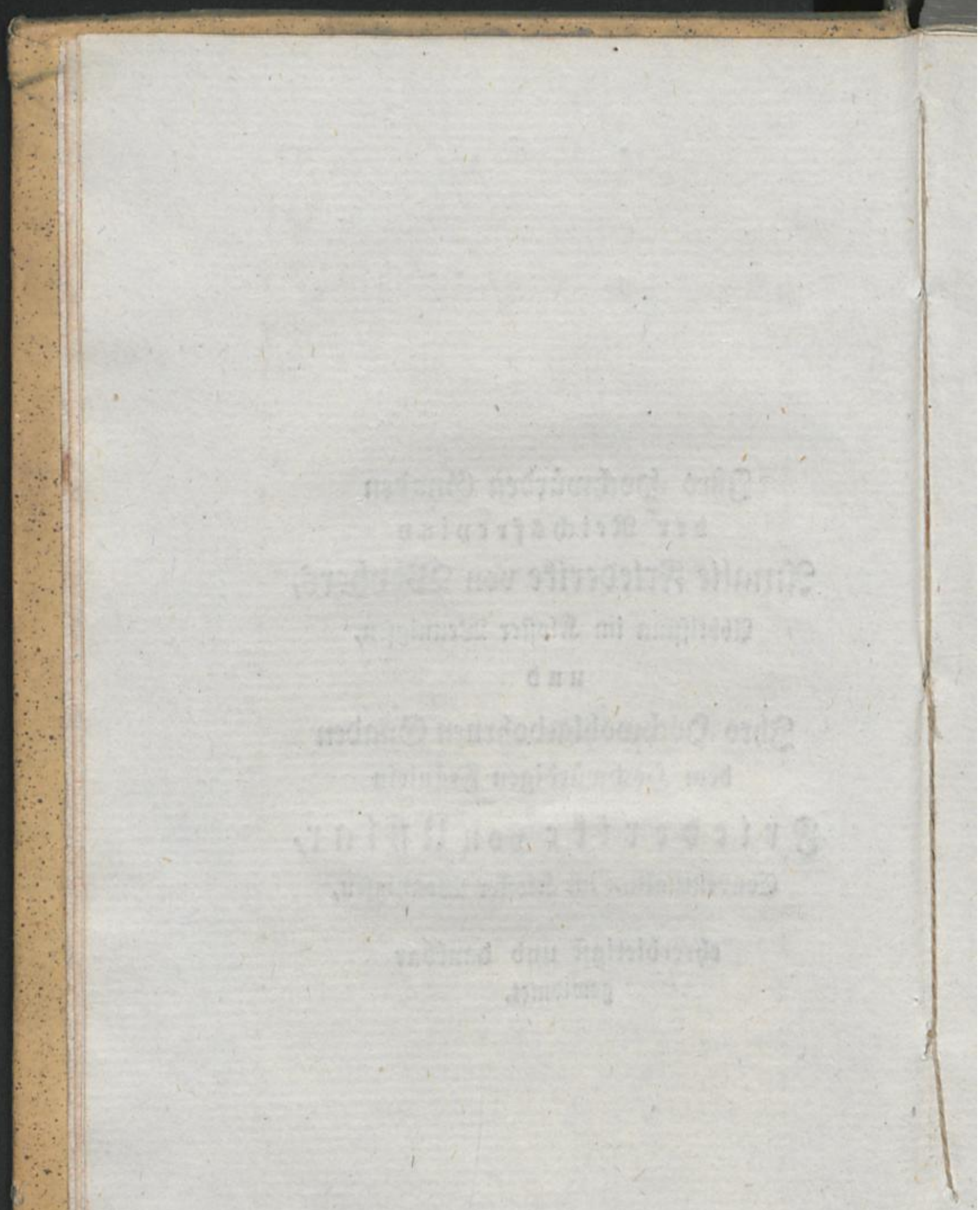




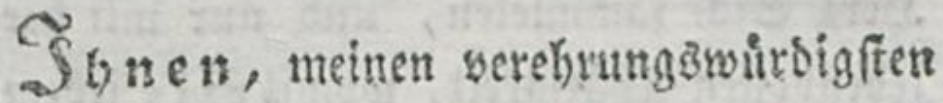
(s)onnerinnen, wage id)'ž, biejen Ileil

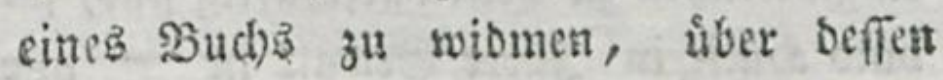
SBerth ober linwert's Riemand vidstiges urtheilen fann, als $\sigma i c$, uno an beffen Dajenn Gie EeIbli zugleid) Den großs. ten 2(ntheil haben.

Pux wenige Mienicten fennen die grofe fdywere Sunft des Umigangs mit Leisenden fo ganz, uns nod) 23 enigere ûben fie fo menf(d)enfreunblid) unb nobls

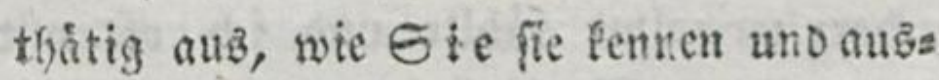
niben. - Nar mit $\mathfrak{I} \mathfrak{r e r}$ feltnen

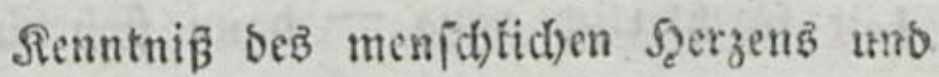
mit $\mathfrak{I}$ bre $m$ feinen Sinn fü: icoe Siotly unb fúr jebeß \&eiben; nur mit ber 3nerts beit uns 28 arme $\$$ b) Der 
Der Falle und siraft $\mathfrak{I}$ be $x$ S Bieiftes,

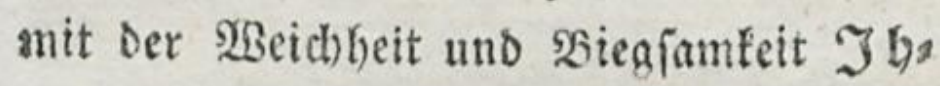
I $\mathfrak{E}$ o ebelmeníf)lichen (Sharafterz, mit bem Reicht)um joldher Erfahrungen und $B e 0 b=$ ad)tungen niber Menid)en und meni(d)= Iidhe Ragen, wie Sie ihn fur Sieift uno Seerz Sidl fammleten, und nur mit Der lebenvolfen zhótigzeit, womit $S$ ie jedem Itnglíctlid)en zu helfen gefdoaftig find, jo= balo Szulfe in Sifrer (Sewalt ift - nux Dadurch wirb mian bem Reibenben Dab, wob ein glictetd)er Troffer ifm werben fann, uno was Gie mir und unăblis d)en 2 ndoern auf bie wolglthåtigfte $\mathfrak{B}_{3}$ eife oft geworben find- - 2(d), wenn id $)$ in fo nand)er feibensivollen nes febens nirgenos a roft fand, wenn id) meine ganze Seele gelátht unb mein Şerz zermalmt fúthlte und id) oft mit fdrectlid)er P(utblofigfeit uno Rebensfatt= beit furchterlid) rang - bann flob ids weg von allen Menichen bin zu $\mathfrak{I}$ h $\mathrm{n}$ n, ebelfte Theilnelgmerinnen an allen meinen Edjickialen! Sie allein werftanden mein

Ieis 
leibenbes ḩerz; $\sigma$ ie allein extrugen mit bimmlifâter S(b)onung uno nie ermúbena

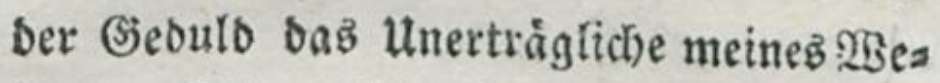
fens; Si a allein brad)ten mir wieber Sidft in bic Seele und Muth ins muthy lofe Sterz - o wie banfend und frendig

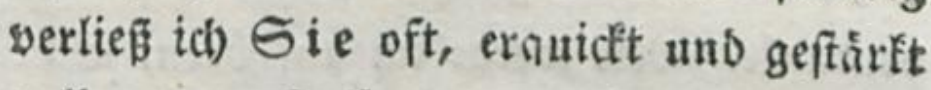
solf neuer Soffrnung, wie neugebogren औu ueuem Eeben! .

Da fount id) von Ibnen bie Sunft bez Ulmgangż mit Leibenden an meinem eigenen J̧erzen lernen, unb habe ich) baher irgenb etwaz etwab 2 Bahreß und Giutes baruber in biefem 2 budse ge= fagt, fo gehoirt bas nicht mir fondern Ihnen.

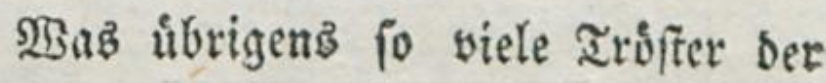
Seibenden mit allem guten 28 iffen nich)t thun unb nid)t $z^{u}$ thun vermogen, $D_{a}$ thaten Gie noch an mir: Gie troftes ten mid) nid)t nur, Gie balfen mir atch), balfen mir auf eine 2 (rt, bie mid) (d)nell unb ganz fuber jene traurigen হage bina 
binweg, und zu ciner ghtlle yon Seben bob, wie id) fie fur mich nie megr erwartet, nie mebi gehofft hatte.

(Sott vergelte 9 g ne $n$ biefe und alle bie unzåtid)en 28 oflthaten, oie tit) Эদ) werbante. Nie werbe id) aufforen, Eie zu fegnen, nie aufgoren, mit jeber (sms pfinoung bes Reben zแ feyn

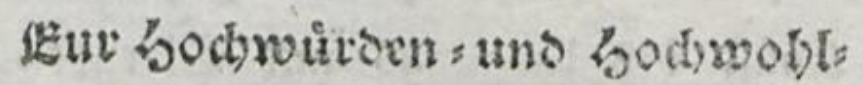
gebohinen Gnasen

innigfi yerefrenser tun banfóter Stieorid) 23 urdh 6 ro 23 encten.

23or: 


\section{รorberidt.}

SYSaz ich Dir, guter Sejer, jur richtigen

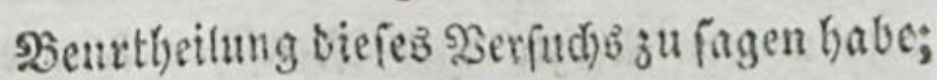
finber ou an Sthlun Der Einteitung, wotin ich Did Dezhalb vermeife. Jith fortieb ifhn anfangs fúr mein Jabrbuds, erweiterte abce nacb) ber meinen भुlan uno beftimmte ifym diefe form, um iffn braud)= barer und gemeinnutbiger z"ll mad)en,

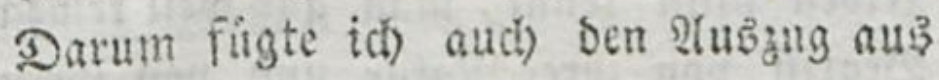

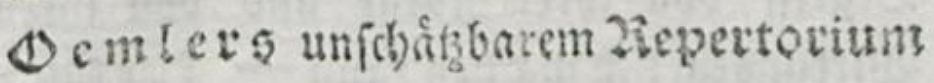

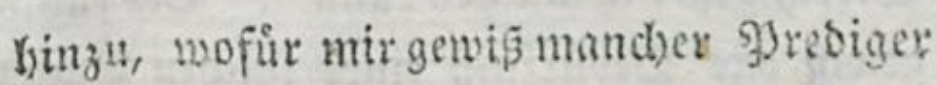
uno mandyer Freuno Dor Seidcnien Dan weiß, oa Dies treffliche, an stmtke exfalyeungen und Penjid)entenntris und

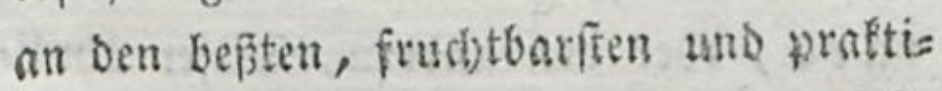

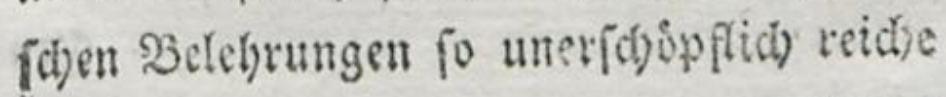
SBE 


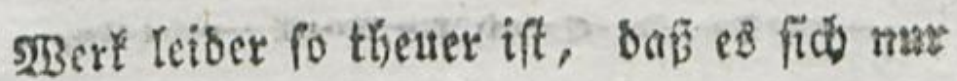
wentge গyrediger foufen fónnen.

Der zwerte Theil biefes Berfuchs über ben Ulugang mit feibenden wirb, auffer mel)rern gefammleten Semerútu gen tiber feibente und ifye $\mathfrak{B e l}$ andlung, bauptfids)lid sine piychologifdre Warbos logie, eine praftifdhe Naturgefdidyte bek meiften Seelenfranflbeiten entlyalten, wo (ä id) Die Sá)riften ter grêfîten áltern unb netuern 2 terste unb Menfden fennex benufizt babe.

ISer ę weißs, waz Eeelenleiber fino, uno oft wie Durd) Yeid)tfinnige ober unweife Behandlung theilnelymender Freuns be ober untheilnelymender Mieniden vers melirt werben, bloz, weil man foldbe Eciben nid)t fennt, man gar weil feinen Begriff son ifyer Patur uno von ifyren SBirfungen fidc) madien fann - Der fúblt

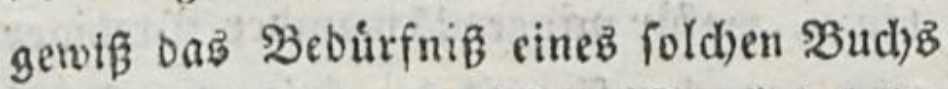
eben fo bringeno, wie $i d$. - Frenlich) follte cin foldbes 3 udd nut von einem 3 ims mermann, Weitaro, Mara caro, ober yon andern, Diefen àtulio dien 


\section{XI}

()en, grof̊en 2tergten gefibrieben wer= Den - uno vieflecid)t brångt fie ilbe eblem für bie lcibende Nenj(d)lyeit fo fúklbareß Şerz einft nod) za biefem fegenşvollen

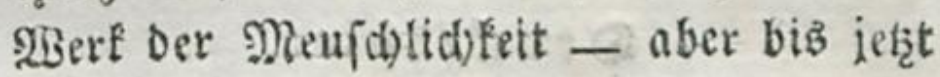

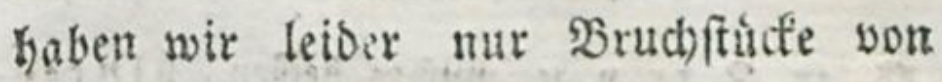
ifnen. Es ift baber gewiß 28 ohlthat für ungiohliche feibende, unb son gropem Nulzen für Seben, ber bie Menfoden in allet? Sagen fabirt, bieje uno álnnlidbe $\mathfrak{B}$ rud fitude zu Einem Ssanjen zu fammlen, unb fie fo von nettem in grókern unb gemein= núkzigern umlauf 孔 bringen.

Id) Koffe zu (jott, baß Ex, ber mir fd)on fo mandbes findlid)e (Sjebetl) erborte, auch Dicfe 2rbeit, zum 2 Bohl meiner lets Denben Briber uno Sd)weftern, gottlid jegnen werbe.

\section{Seneten.}

Nonnenberg bey . Sannover. in November 179r.

Drud: 


$$
\text { Drudehlex. }
$$

c. 32. 3. xr. v. แt muk es beipen, biutene De fintt blutender, uth 20 und wegzufireid)ent.

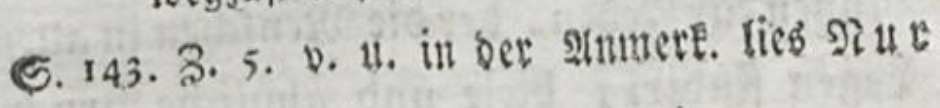
ffntt sididt.

Die íbriden unbedentenbern Drucffeftee switb Der geneigte gefer Serbft bemerfen uno serbefiern.

Universitäts- und Landesbibliothek

Sachsen-Anhalt

urn:nbn:de:gbv:3:1-840212-p0016-2

DFG 


\section{Lleber Den llmgang \\ mit \\ $\&<i 0 \pi$ i}

Sak ben SBeinettoen nidit ofne stoft, forbeen traute mit Den פraurigent, beidswere bids nicht, Die firanten a befuben, Dent unt Deswillen swirft ou getiebet werden.

Sirad。

So ifi eine ber füfeften uns beiligfter פenfidenpflidten, Reibenden aller Irt fich mit Theilnabme uno liebe zu nábert, פefummerte zu berubigen, Sd)mad) zu fiårs fen, Niebergefdiagene aufzuriditen, firante.

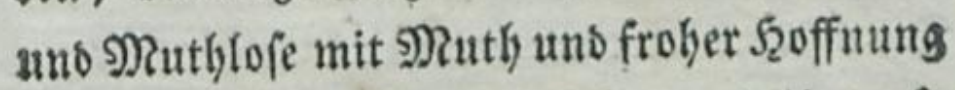
ơ beleben, und unfern llmgang mit ifnen fo soblylthátig als móglich fúr fie zu madben. (E⿱ ift fo mentchlich) und fo góttlich, uno

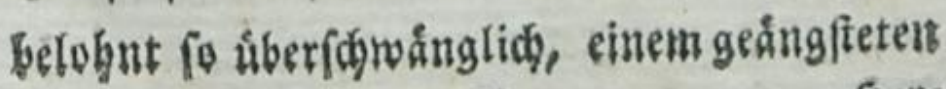
\& Sgets 
Serzent, sab alle Freute uns alle salke ver Iofyren hat, Das nirgenso srofi findet uno

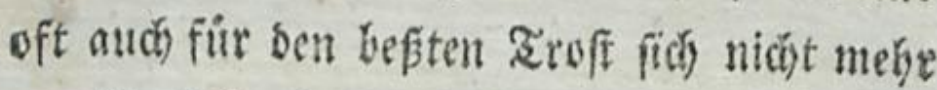

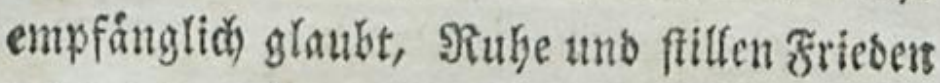
sieser ju geben, uns Dem, ber bilflog wers oagent lange aflein oa fans, unb fich verlaffen glaubt yon sflen uns lieynabe figon serzweis felt an झllem, ein Engel ber Stärung unb beb รroftes zu fern, uno ibn burch unfre bribers fiche Theilnabme mit Menfchen, mit Echictfal zuno - mit fich felbft wieoer aubufushnen.

Iber frenlich if sas oft fo fowmer! Uno nutr wenige Szenchen verftelyen fich auf biefe grope forwere fimfi, fo willig uno geforaftig auch) Die शeifen fino, fic ba auşzulben, wo Senfzer uns Zhranen fie zum \$itleid ribren uns zu mitleibiger sheilnabme aufforbern. (E) gebort mebr bazu als ein feerz yoll guter 2Bbinflye uns woll guten 2 Billens - reidyte. D) ล b bin, ficher wưtie man bann bie \&eiben. ben nicht fo oft Elngen boren über Julbllofigs feit uno Şårte Der Menfchen. Der Bormurf Der Unem $₫$ finofich fe it trift gewif bie sgenigften. - Šeder gute, jedes nod) nidgt

gan 


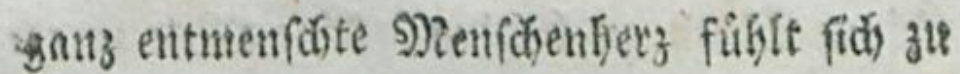
jebent Reibenben allutadytig bingezogen, wo ę ifn finoet; Die Menfógeit regt fich in ify

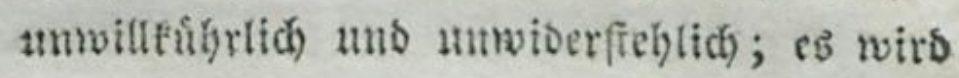

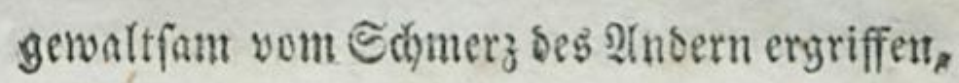
es leibet mit, wo es leiben fiegt uno hórt,

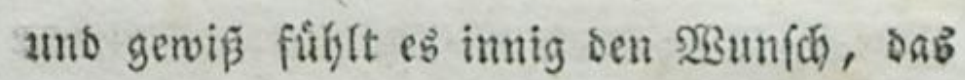
Sciben yon bem Alnglietliden binweg ju nelys men, menigfens ifm linbern zat fómen. Stber - weil fo viele Mceuf(cen bas nidft zut yetmigen glauben, weil fie fich bazu nidjt Siraft genug zutrauten, uno weil eb ihnen, befonbers in unferm weichen empfinsfament Beitalter, aud wirelidy oft an Sirnft und Ges

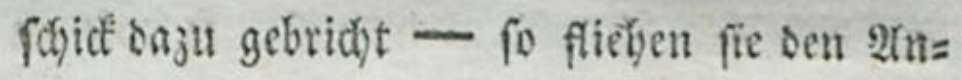

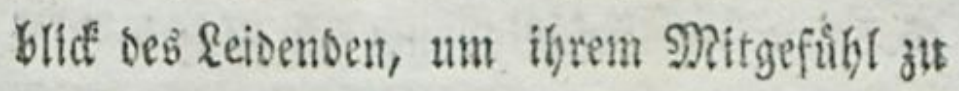
entfliehen, uno - fo ift ein weifer Trofta

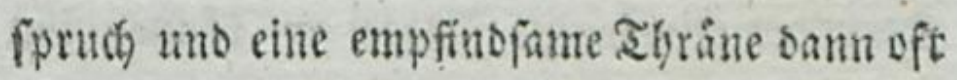
ser ganze Iribut, Den fie ber Menfdylidfeit opfern.

Inbere fuden sem Reibenden zwar Megk su wersen, fuden surch wabre Ifeilnabme, Durd) Sghjilofophie und 23 ibel, ourch eigene

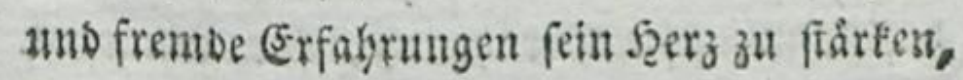
$\rightarrow$ स 2 feinest 
feinen Nath zu beleben, feine Sele su en beitern; fie weichen Tag unb शadbt nidyt yon ifm uno bieten 2llfeb zu feiner \$erathung and zu feinem Troft auf, und bods ift 2ulles3mronfít Reins ihrer gutgemeynten 2 sorte sno feine ifyrer resliden Bemůthungen oringt iłm ein in bie Seele - fie martern ifn mur suit all ibrer zuturinglicken Theilnaknte uno er fehnt fich weit weg yon ignen nad cintm fitllen einfamen Drte, wo ihn fein Trófter quált. 2tch - fie baben ja nidyt felbft ges

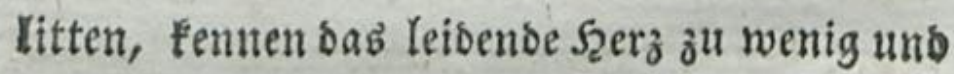
begreifen ia fo wentig von bem, wie ihm eis

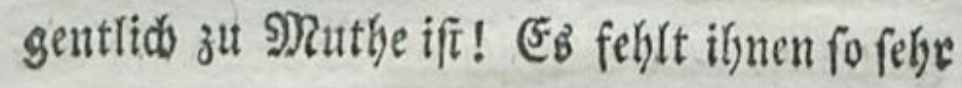
an richtiger Menfchentenntnif, fo wie an Jeinbeit bes Gefübls, um fid) ganz in reine Eage, in feine Empfinbungen, in feine Dens fungbart binein zlt fitten - fómen ihn barum fo wenig beurtbeilen uno fo wentg ibm gebent yon bem, was er eigentlich) bebarf; fie balten feine Silagen, feine Thränen, feine

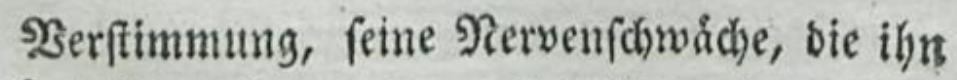
jezt fo reizbar und empfinolich madyt fur bie ไeifefte un!anfte Seruigrung, für \&aunerey,

हैi 


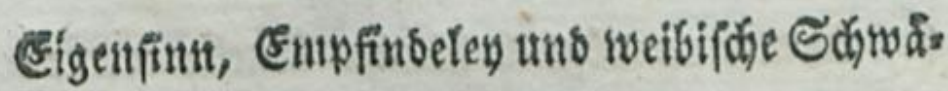

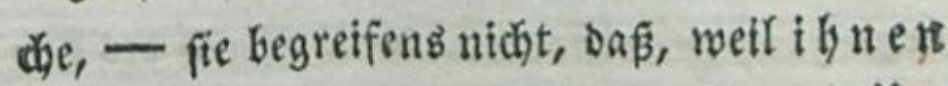
Dies uber Dab fo wenig Sdmerz, fo wenig Uln sulje, fo wenig Sorgen madst, es irgeno eis nem 2 ubern fo fdumerglich und peinlic fent tonne, uub darum behanbeln fie ifhn mie all ibrer siebe boch oft mit fo menig Sdyos sung; fie forbern oft fo unbillig, baf iebes

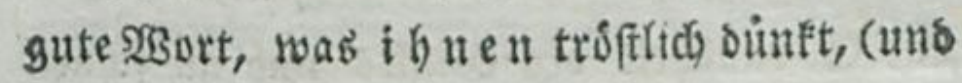
was bod in oer That oft ber frafts uno pafts lofefte (Bemeinfprudi) ifi) $\mathfrak{X}_{3}$ under an igm thun, ihu fein Elento vergeffen lebren uno ihu ůberzeugen foll, ons er jezt ketnen Sdhmers mebr fiible, fo laut auch fein $\mathcal{J}_{2} \mathrm{er}$ und alle शerven feines $2 B$ efent Dagegen farrenen פBas follen ifm baber foldye Trofter mit all ifrer zlyeilnabme uno mit all ifrem guten

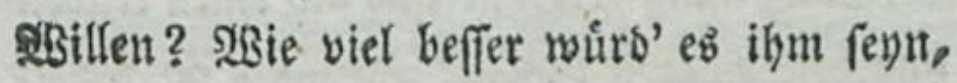
wie viel weniger witro' er leiben uno wie viel fóneller fein \&eib vergeffen, wenn alle bie leis sigen Trofter von ibm widen! -

भuch bem gefdidteften Trsfter wits $e B$ oft fojwer, unb nicht felten ummoglid, Troft in ein troftlofes .5erz zut bringen, weif eb ifnt स 3 fafि 


\section{6}

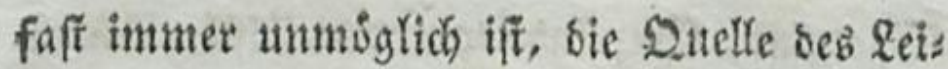

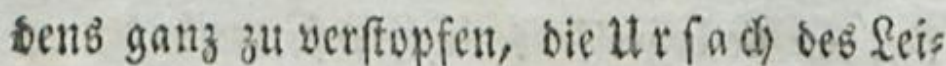
senb, bie $e B$ unertriglich madht, ganz binte megalchaffen. Stber handelt er mur immer mit Slugheit uns Riebe; weis et fich nur bas yolle bingebenbe $8 u+r$ a uen bes Seibenben

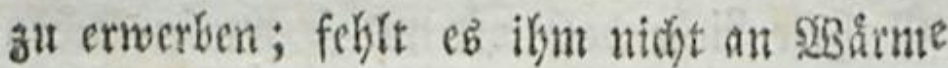
uno jarter F⿱艹 sub Bjerwanbheit bes Bieiftes und an fidnell auffaffender uno richtig treffenber Nenfchens Penntnif́, um jeoen feibenden fo zu behant= beln, wie grabe ein for cher Reibenter mit foldhen Geiftestriften, mit foldger गुerzents fimmung, in folober Iage uns unter folden Limftánben bebanbelt werben nus; lat er fich mit ben Quellen bes Zrofes uns ber $\mathfrak{B e s}$ rubigung innig vertraut uno fich einen reidben

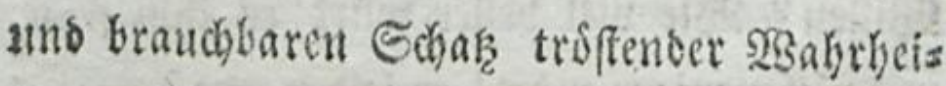
ten zu eigen gemacht; bat er's felbft erfatgs

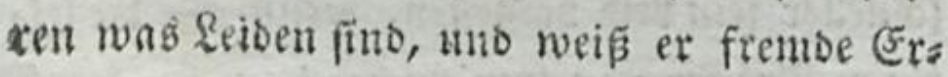
fabrungen nit 2 Beibheit zu nuken - bann soiro es ifgm mit (sott bod) mefre gelingen als mislingen, uno manche Freubentbråne, mancher Jergensbanf und ber ftille Segen bur(t) 


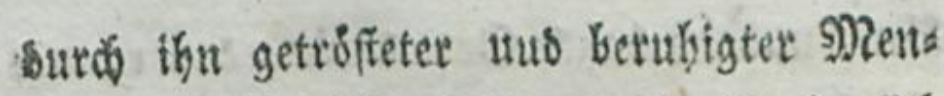

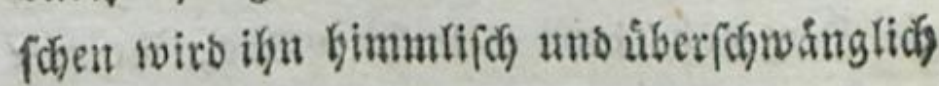
betohnen.

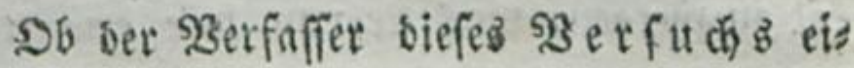
nigen Beruf bat, Ettvab uiber ben Uningang mit \&etbentoen zu fóreiben, bab riberlápt er bent

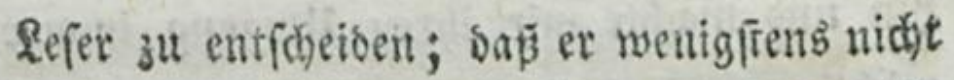
ganz unbetannt mit dem leibenben Szerzen iff, unb manche wern gleich nidast nette bod rich):

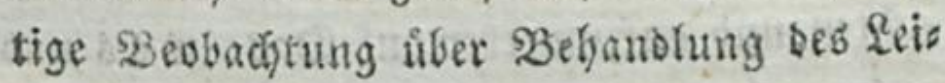
Denben aแb feinem Erfabrungsfreife gefamo melt hat, gegen bie nod tula áflidy oft voit Freutben ber seibenten gefehlt witd, Dies wiro man igm hoffentid) jugeftefer.

Bon jefer futfrten mid meit edbichat unb mein feer oft unter feibenbe mancher 2rrt, uno won leher interefietten mich \&eidense mekr, als Gluctlige. Sdyon als Sinabe zog mich oft eill trauriges (sefidtt weg vom Tums melphą jugendliçer Fretten, un wenigftens Ffym Mritleid zul beweifen, wenn ich suth wei=

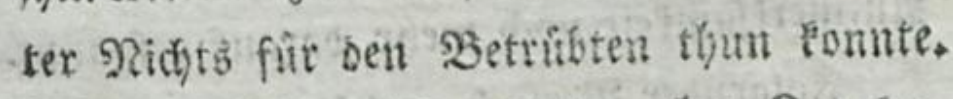
Dft war id in reifen Safren bey STranfell,

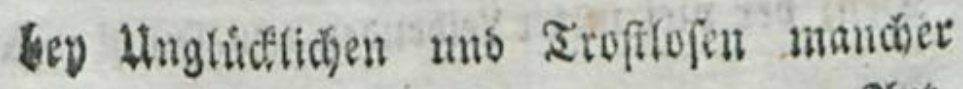

$$
\text { I } 4 \text { Irt, }
$$




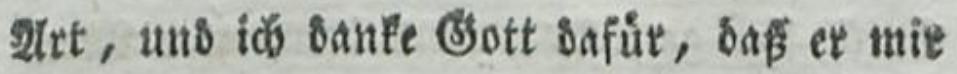
ein Szerz gab, bem es leidyt wurbe, ibr volo Iอร 3utrauen zu gewinuen, und ifnen Das oft zu werden, was fie von mir erwarteter sno was id ifnen werden fonnte. Dies trieb mich bann naturlich nod) mebr zu ibnen bin uno machte mir ifren llmgang immer soidtiger and lieber. - EB foblte mir bas ber nidbt an Belegenbeit, Leibende zu beobs achten und aufzumerfen auf $\mathfrak{D a b}$, wa w te auf fie gewtrft werben mus, uno was uno wie von bem grofen unweifen \$rofter= trós gewoiknlid auf fie gewirft wird. Fis gene, båuffige, nich)t felten tiefbeugende \&eiden

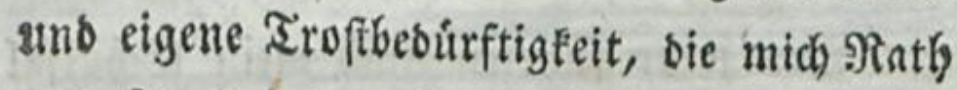

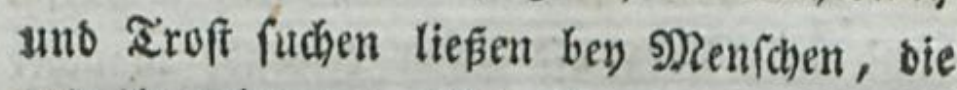
mir ihn geben unb nicht geben founten, baben mir diefe $\mathfrak{B}$ eobachtungen beridotigt, beftátigt uno vermelyt, uno ba mir jezt mein 2 (mt ben Hmgang mit Reibenden zur soppelt beilis gen und fúfen sुoflidgt madjt, fo if meine 2Iufmerffamfeit nod) mebr barauf gefpannt.

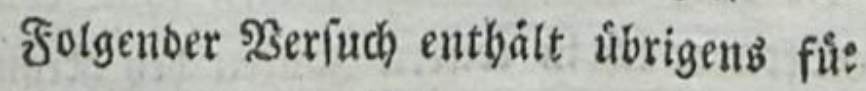
Den, ber viel unter Leibenben getvefen iff unb 


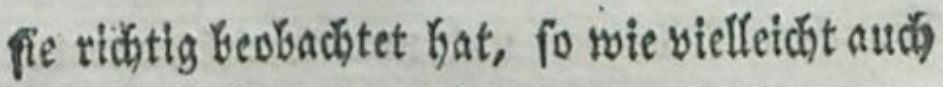
feron für Den, ber bie feinern Iflidten bee Befelligfeit uno bes Ltmgangs fenut uno íbt, uno bem eb niajt an jartem Gefúhl uno Remfchentenutnif fehtit - wenig ober viels

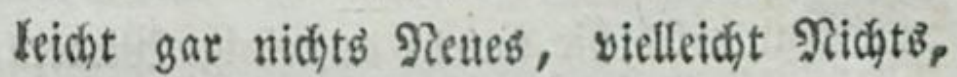
wab er nicht fogon felbft bemerft unb geifbt bat; aber - fuir Diefe if biejer 2 (uffar aud nicht gefourieben; furt fie if beynabe jebes

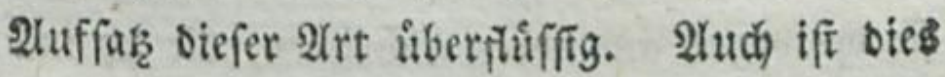
Ganze noch fehr unvolfemmen unb mangels

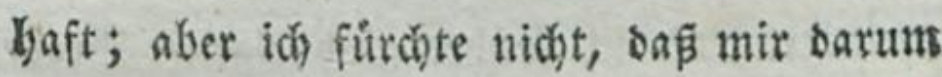
billige Beurtheiler bie Szerausgabe biejer Edhrift zum \$erbredhen maden werben. Sollte Der, Der bab bringende \$eburrfnip eis

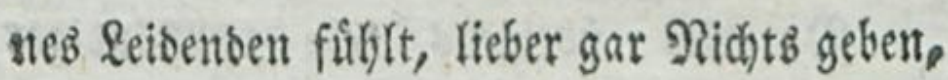
weil er ibm niđt Illles geben faun? - Neir er giebt mit gutem frerzen, fo vieí er vermag, and wüncht unb bofft babey, sap reidere Denfchenfreunbe Megr fút ben Sedaurenss serthen thun werben. Sid gebe barum bier, fo viel id) $f \cdot a n n$, unb bott weís eह, id gebe

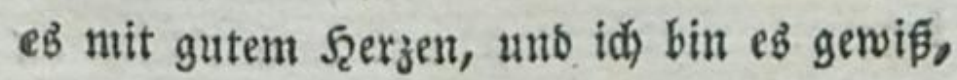

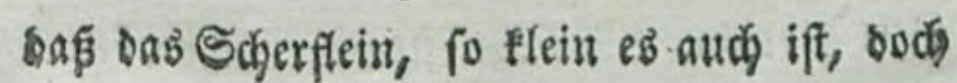
24 5 matrs 
manchem Leisensen uns manchem freuns set Seidenden willémmen feyn wirs uno nusen fonn, uns diefe Hleberzengung beftimmt mich

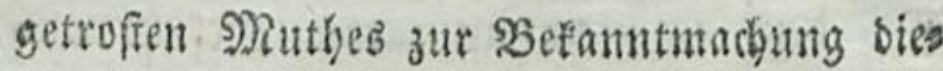
fes serfuctso.

2) Man wirs bie uns ba auf einige 20ieber. bofungen frofen. Dieb formm onker, weit ich aud bie Seobachtungen grofiserer Senmer Ser Leidenben benusen wollte; uno idh fibjte fie beswegen aus ifhren Gibriften, bie id ime ner angezeigt babe, wortidid an, um bie

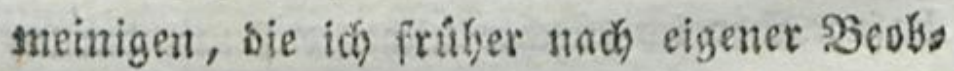
achtung fammelte uno nicberfarieb, eb id jene fonute, zubeftatigen, uno ignen baburdi melgr $23 e r t h$ uno Sraucbbarfeit zu geben.

Da bies uibrigens tein vollfánoiges S̈y fiem feon foll, wie Reibenoe zu belgan= seln fins, in bem yon Stuffe zu Stuffe dee S2Beg gezeigt watre, ben man mit ifnen gehen mus; (mie baun wol hbergaupt fein foldjes Eyftem gefdhrieben werben tann, weil jebec Sirante uno jeber Reioende auf fe ine 2 Beife

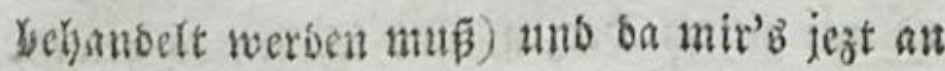

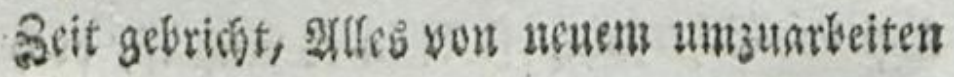

$13 n$ 


\section{II}

anb gentuter zu otsuten, fo fogreibe id me me einzernen \$2eobad)tungen Gin, wie id fie mir aufgezeidnet jabe, whue fie intrfflid ju orbs sen und zu freflen, was iấ bier furr äberfliff fig balte. Seber, ber fie brauthen will, wirb. and mus fie fich in ber Soronung hernub fins Den, wie er fie brandt.

ennonionm

gुsillt 
2Jillfit but sich bem ltutgantg bes Seibenbet

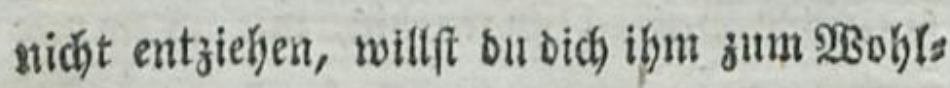
tháter uno jum Şebirfnis machell, foll er bir ganz trauen unb auf jebes beiner 280 orte bơren, uno willf ou fo auf ibu wirten, wie ein glúclidjer Trófter auf ihn wirfen taut, fo

\section{r.}

Fey yor allen Dingen ein recht thaffes ner, guter, Iiebetbätiger nien \{ch, obne Eug uno Gelbfrudst, obme leigennus uno nieorige Leioen ibaften.

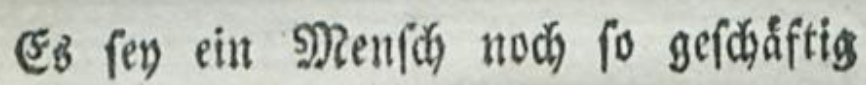
midy in meinem seiben zu berubigen; er stánge fich mir nod fo nabe uno beweife mir Peine Theilnalgme auf alle $\mathfrak{Z B}_{\mathrm{B} e i f e}$; er weine in meine Thtånen uno flage in meine Sllagen; ex rebe Stunden= und Tage lang zu meinem

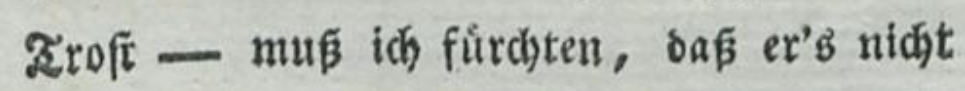
reolid mit mir meynt, bás er anbers benft, als ex fprid)t, baf ex unter ben 2(eufferun= gen feiner Theilnabme noch beimliçe Rebens abfichten verbirgt: fo erfigspfe er alle feine siråfteg 
Sirafte, fo bebaure uns beflage er mid unb

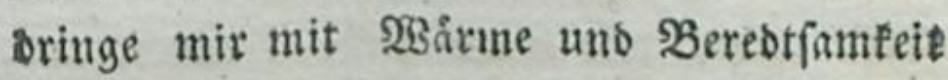
alle Troffgrinbe ber হsernunft unb $\mathfrak{B}$ ibel auf - in , meinem Snnerfen wirb fich bod immer etwas regen, was laut Dagegen fprid)t, wab meine Seele ganz von ifm entfernt uns mein f̧erz gegen alle feine 2 Sorte uno ges gen all fein Thun verichlię̧t. Şe nåher e* fich an mich Drángt, Defto Drifdenber uno

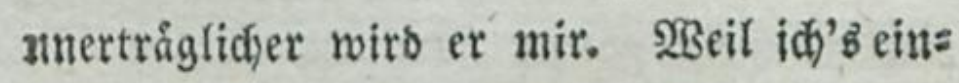

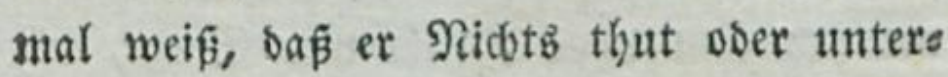
lápt, wobey er nicht auf irgend eine $2 \mathfrak{B e i f e}$ feinen Ssortheil fucht, fo ziebt fich mein gans

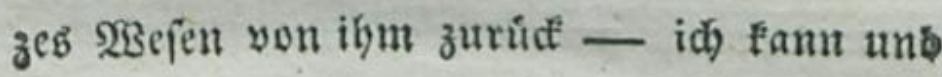
Darf nidjt offen gegen ihn fern, mus grabe sie Sgunben, sie midh am tieffen fomerzen, am forgfältigften yor ihm verbergen, weil ida immer fuirchten mup, oá̧ er meine Dffens beit uno mein autrauen mifbraucben wirb.

शfer eह foimme zu mir ser Maann son

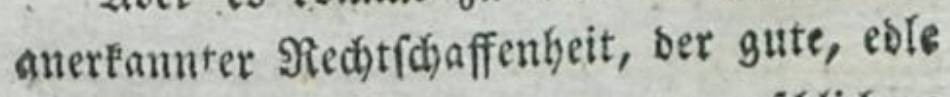
Senfol yon grabem Simn uno menfd)lichens

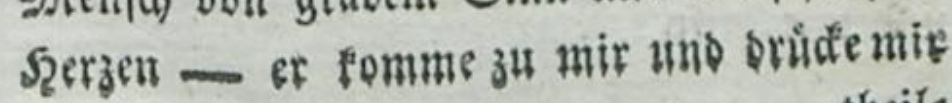
theits 


\section{$\$ 4$}

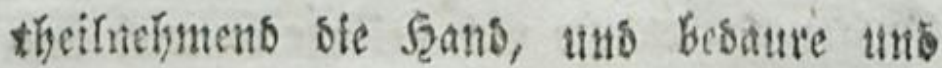
troffe micts, wie trette Ricbe bebauert uns troffet, uno - all ntein SBefen wito ifm entgegen freben uno burch ifn fid getyoben fuiflen; fein Seers unb feine ungetuinfelte Szerzentiprache werben mich innigf rifgret, unb jebes feiner 2 şorte wirb nuf mich wit= fen, wie ein milber frud totorer Segen auf Dab bitrre Eroreid). Uumerflich uno Teife

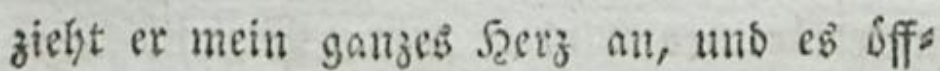
net fich ign ofine Furdyt tmo Sorge, un

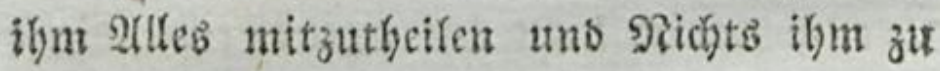

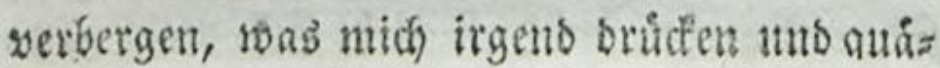
Len mag; idf borche gern auf feimen 3 at? fprutch, auf feinen Troft uno auf feinest treugemeynten $\Re_{\text {nth }}$ - uns fieffe! meine Safrainen verfiegen, meine saft briakt mich weniger, mein Seerz if rufiger und id füble mich wiebergeboren yoll neten Nutḩ yoll netter Şoffiung uno netten Rebenb, weil Er, ber Esole, Theil nimmt an meinem Qeiben, mid beboutert, mid trơfetet, mir rát uns mir birft.

Dorits 


\section{is}

\section{Dringe Sich Feinem Leibensen mis}

Insisctetion auf, am wenigften baiut, wem ou fein Sutrauten noch ni(t)t haft uno Dir erft erwerben muft. Naffere bid ifm

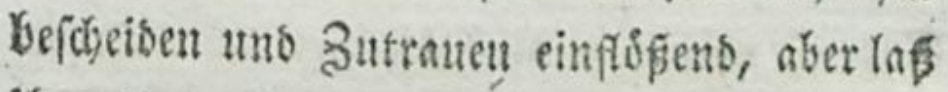

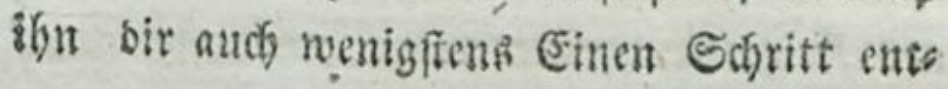
segelt foumen.

Es gibt Reibenbe, bie fich igre feiben gern abratben loffen, bie nidit barum geo fragt feyn wollen, umb sie eb immer yote aub ferzen, bafi man fie foud wiffe. Eb giebt Sindere, benen man fie bis auf ben tleinften 1 Imffanb abfragen mus unb bie fión firt beleibigt balten, wemn man fie nidgt ges swiffermasen zum Steben jwingt. SBer bakee ๑ab Butrauten viefer \&eibenden gewimnen will, der muf es wiffen, of uno wie uno rie weit ex fich) ifyen neibern barf.

Ë giebt Leiben uno \&agen, sie mat ungern, fellof feinen vertrauteften Freunben, geffehet, bie man lieber im Etillen trägt, unb Deren Pittheilung man fich (d)fed)tersing nicht absringen taffen fanm.

Noit 


\section{6}

Mit mandiem Reiben mus ber Leibende erft felbft ganz vertraut werben - er mus fie exit ganz liberfefn uno ourchfiblen, uno é̉ erfi ganz empfinden, baß̃ er Durchaub frems

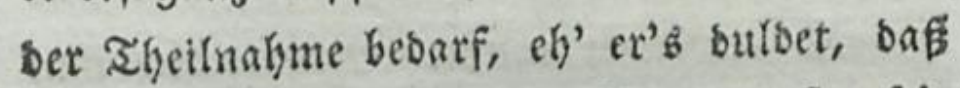
Indere, wairen ę aud bie \$ertrauteften fei=

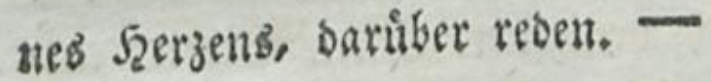

(e'z giebt Leiben, bey benen bab Jeerz fich

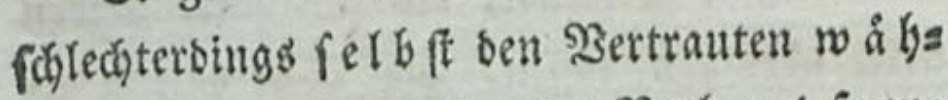

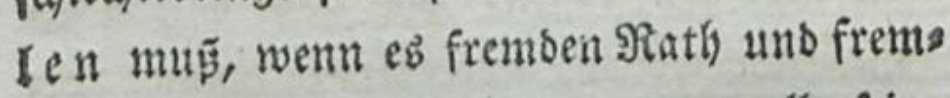
sén Troft bơren will. Nan fann alle feine Freunbe innigft lieben, man fann yon ibree

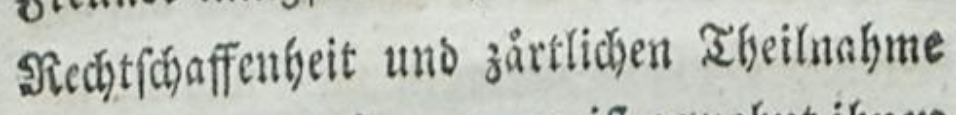
ganz uiberzengt feyn, man if gerobhnt ifnest alleb Inoere mit ber uneingeforinteften Dfs fenbeit mit zu theilen - nur grabe über siefe lage, luber biefes Leiben ift $\mathrm{eg}^{\mathrm{a}}$ uns unmogliă, mit ifnen zแ reben - Daв tann id) unter glllen nux mit Dem, zu bem $\mathrm{i} e$ zt mein Szerz midi brangt - und felbft die befs= ten liebfen গ2) Iid) martern, wenn fie fich in foldhen \&agen แแถ gewaltfam auforingen wollen, oder men! 
fie unfer Edyweigen gegen fie fur Nanget an Sutroneh, furr erfaltete Frembfchaft umb ans redynen; surth foldges 2huforingen gewimnen fie शididt, weil bas hetz bam am wenigfent

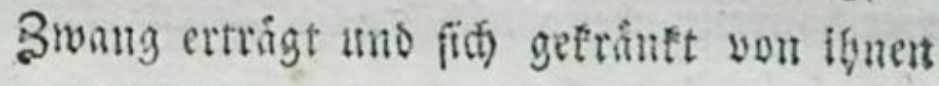
zurúd jiebt.

bande saber immer mit Discte= tion uno feinem Gefribl, wern bu bent feibenten nicht mely thyn uno vir fein 3utranen

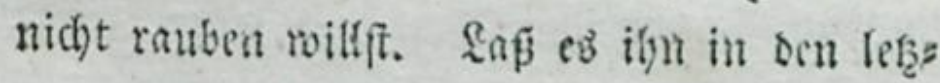

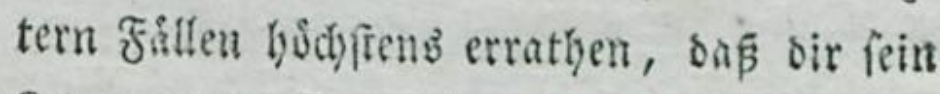
Reiben niçt gans unbetannt if ifu fublen, sap ou ben berzlidfen Intheil Daran nimmi - nábert er fid sir bann niobt, fu gefje wentigfen begt nod niçt weiter. -

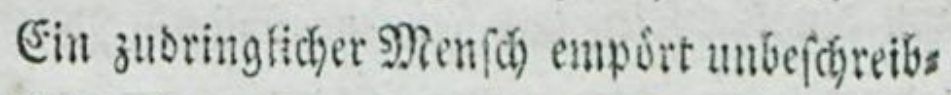
lid) in jeser \&age und anter allen Innffintoen, abe: bem Reibenden if er gar entifiglich.

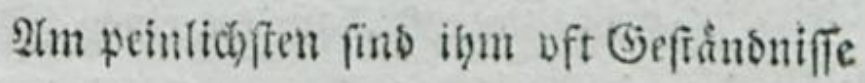
foldyer Reiben, in bie er fidid ourd) cigene Thor=

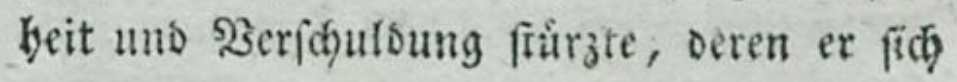
jęt fogint. - Siamift ou onber ify tróffen ano ibm beffen, obue ein fold̆es Selbfigges frănonis, fo erfpare ifgm die peinliçe şerles 
genbeit unb forbere ebs nidgt yon ifm fcheine bie Utrfach feiner Reiben wo inóglidg gat nid)t zu femen, uno thu, in Stillen, wab ou fur ihn thun fannft. Se feiner ou Gier Gandelfi, befto hober wiro cr bir's an= rech)nen, befro mefre gewinnft bu fein Butraus

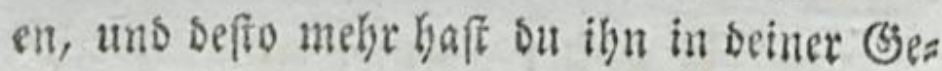
walt.

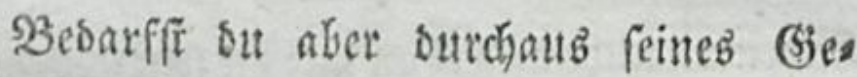

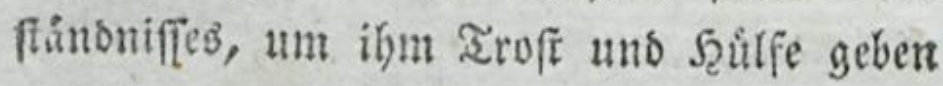
zul finten; fo serfabre baber) fo fdouneno als moglich, uno Ia $e^{2}$ ihn vor allen Dingen auf alle 2 Bzeife fuffen, saf er saburds an bei= ner 2lditung und Liebe nidfte ver: Iobr - baf bu Nenfdenfemer genug feyft, un ben Menfdyen unb feine menfdylichen 2 sers irrungen aus bem rechten (Sefichtspunft bes traditen zu fónnen, und fie fur Das zu nely. men, wab fie fino - bn (d)en nicht nach e inzelnen franolungen, Fondern nac̆ feiner $g$ a

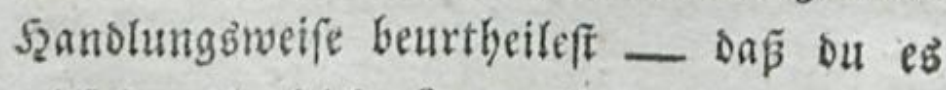

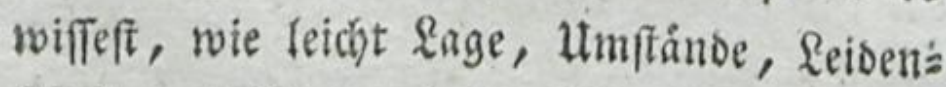
fidaft ze, felbfe ben beften MRenchen zu Dins 
gen verfeiten Fonnen, die er felbft verabfódelte et - oafs barum butch (Fine folechte, felbfit burdb eine abfdyeulidge frandlung ein guter Menfh, oer vorker beiner \&iebe werth war, noch nicht zum (d)led)ten abfibeuliden SRena fiden werbe, ber mun beine liebe nidbr melge verbiene, *) baf bu felbft folder Toleranz bebirfef, uno fie darum nod) weniger beinen Freunben verfageft $2 c$. - Er wirb bir bann Defto williger und frober entgegen fommen, uno mit einer Sffenbeit uno Suverjicht att bir bangen, oie bich in bie gebeimften Ticfen

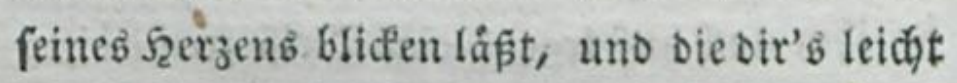
madit, ben গath unb be n Troft ihm zu geben, ocfien er grabe jezt am meiften bedarf, und ber am fickerften uno wobltbátigften auf ibn wiret.

2Im allerwenigften bring bid) einem \&eis benben in futden fållen zum Troffer auf, wenn er irgens etwas wiber bich bat, worú \$2 2 ber

*) cf. Mrofes, Davis, pettus, bein: rid) IV. Ziounfeau, uno - guter $\mathfrak{L} e$ ?

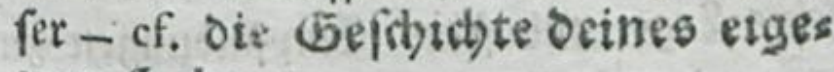
nen Lebens. 
ber er empfinslidy ift, wenn er you bir beleiz sigt ober gefranet ift, ober fich beleisige glaubt 4c. Du magft es gut meynen mit ibm,

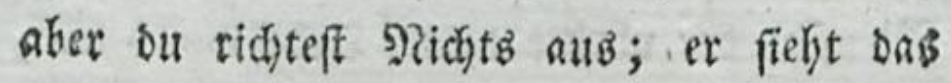
leiç) für Superioritat an, sie bu bir basurch aumafeft uno sie ifn benuthigt, von neuem beleidigt, trinet, unb ifu ganz won sir ents fernt.

\section{3.}

Enthalte bich liberbaupt alleg నrofteng sunb alles Sinthgebens, fo lange ber Unglucis riche noch fein Butrauen zu sir bat; fo lange

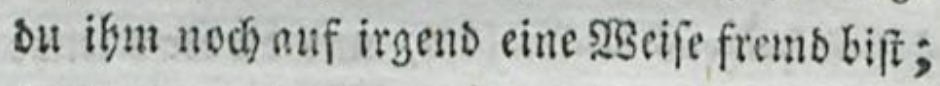
fo lange er giauben muí, oafi du ben llmfang feines ¿eidens nod) niçt fennft uns noch nid)s fenuen fannfi; folange er sir noch) nidjt ivatjs

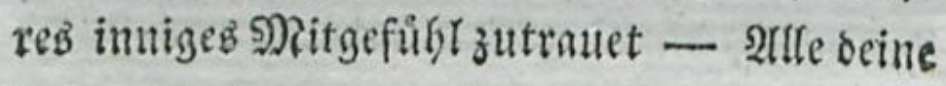
befien Sroffworte emporen fonft nur, fiatt ju berubigen - er wirs sid) fúr einen faben

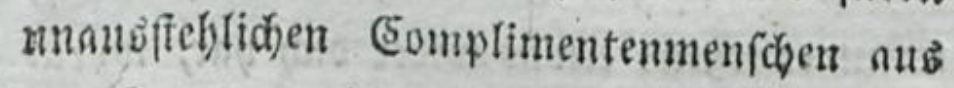
Dem Trof ber leibigen 2ultagstrófter balten, ober fur cinen Nenfden, Deffen \&eichtiun ober beffen diớbáutiges Scers Durchaub unfåbig ifi, $i s$ feine Rage uns feine Empfinoungeu zu bes greia 
greifen, uno nie Butrauen ju sir faffen, uns (ich) an jeoem anbern Drt, mur nid)t in beiner Biefellichnft, ertráglich rubig füglent. -

(5: if unbefdreiblid, mit weldyer lunbes fdecibentheit fich oft unberufenc Trofter uns unweife Sinthgeber zum \&eibenben Ginorangen

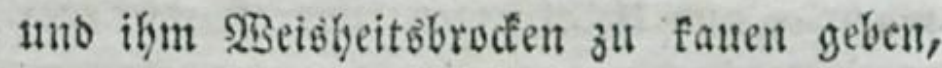
Die fúr ifn ganz unverbaulid) funo, bie feik ganjeg $\mathfrak{x}_{3}$ efen in 2 fufrubr bringen, uno bie e\& grabhin bemeifen, wie wentg fie feinen But fans fennen uno um feinen suftand fid bes túmmern.

4.

Man ift nidjt zu allen Etumben fábig mit sem \&eibentoen umzugeben uno fich ifm wohls thatig ju madjen. S) Ran fagt ifun entweder

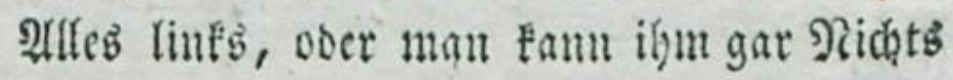
fagen - uns sod) will man ifju butdjaus ets wab fern, unb oas ferte bam in Epamung, in 23erlegentycit, in Hubehagliđffeit, sic unb uno bem Reibenten peinlich uno oricteno wirs, bie ifn oft nud) mutblofer uns angfflid,yer mas d)en, uno woourdy wir ibn auf jeben Jall you uแs entfernen.
23 3
3 Ieib 
23 leib von ifm in folden Gtunben ser Croctenbeit uno Stumpfbeit, went

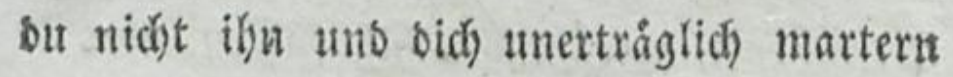
willf.

SBBer einen ¿eibenben berubigen will, ber

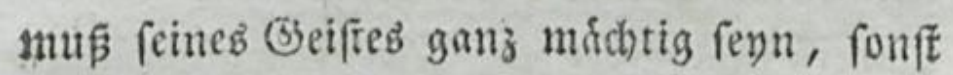
mad)t er nur llebel årger; SBer ftottert ober fchweigt, wenn er reben foll, wem vor 2es

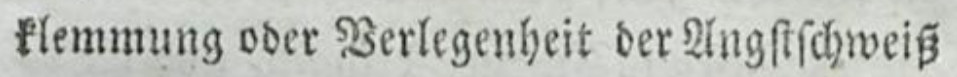

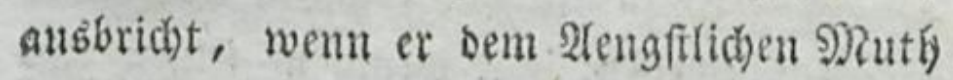
einreben will, wer barmberzig bie Ad)feln zudit, weil feine Befinnungseraft getabmt if ,

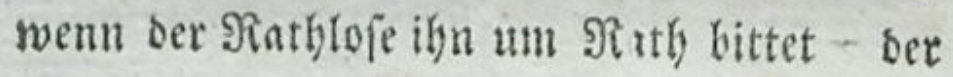
fann voll bes berglichften Natteidens, voll

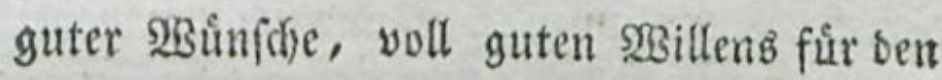
Reibenden, aber wabrlid nicht fein srofter feyn.

$$
5 .
$$

Cabele nie die erfen 2ugbriode ses Eamerzes, weser surd nort nod Zlict uns - rey nidbt voreilig mit seinem Cropt. ミeybeb beleisigt uno cmport unbefdoreiblid, uno entfernt ben \&eis benben augenblictlich yon แแb.

T3as 
2BSiren wit gans bernunft - pagt

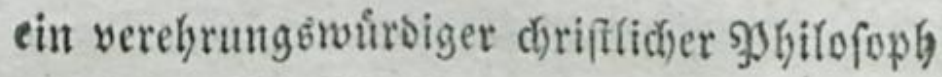
Itno feiner Menfdenfenner *) ober wáren wir ganz Ђ̆ lauben, fo witben wir แแร oft fegr anders in unfern seiben nelgmen. 2rber wir baben autd ein fithlbares empfindlide $\mathcal{S}_{2} \mathrm{er}_{3}$,

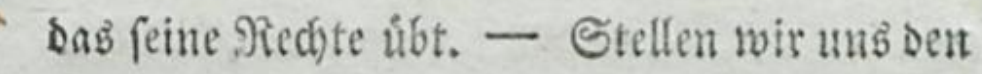
SNenfchen mit biefer feiner \$sermunft, uno Den Egriffen mit siefem feinem Bjtauben yor, unb laffen ihn in Bebanfen burnad hanbeln, fo glauben wir leidgt, ifn forme uno muiffe

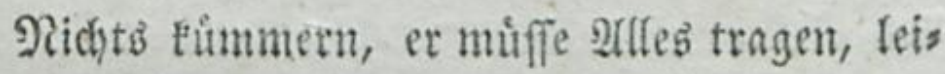

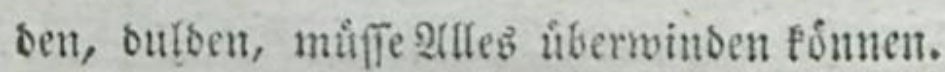
2idfts tóne uno butrfe ifn ath feiner Faffung bringen uno bie Piulhe feiner Seele froren.

Die (sejŏbichte will uns and manchent

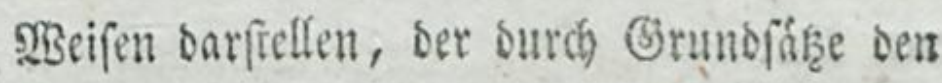
Sdhmerz Liber ben \$̧erluft feiner Sinoer bes fiegte, uno ifhn burd ben Geobanten, er batte in ignen Sterbliche gezengt, gewebrt batte. Man bat auch Troftgründe genug erfunben, sie eine beilende Sraft baben follen, bas (j)cs $\mathfrak{S}_{4}$ mutt!

*) Goppenftert - S. Tefus uno feine כeitgenofien ar 3 . Jammoy. 785 


\section{4}

mutb unter sergleichen Ituglice uns qeiven in

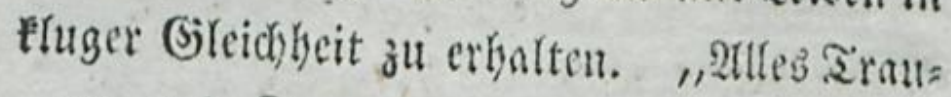
"ren unt Silagen nuß̧e body nid)tb! Nan "muffe mit (siesurs ertragen, wns-nicht 3 u "Anbern ferje. Der Summer föbob. Sian "zeige menig Nenfdenmutbe. (5's zeige we:

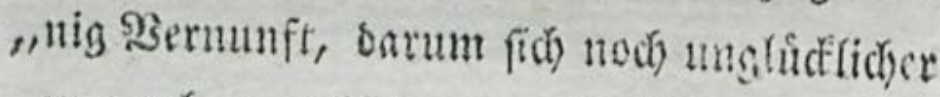
"з3 machen, weil man unglidelich ifr, fo wie „es thorigt fey, bey Dem Serfuft eines Theils "Won feincm Gute, fein libriges but nutb "weg zu werfen." - \$an erwartet 23iel

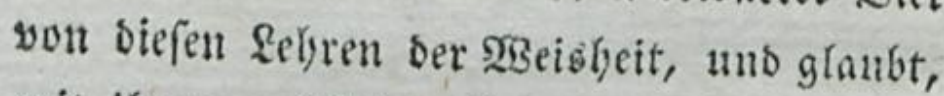
mit ibuen geftäblt, forme oer SBbeife jesem unglưd হroş bieten.

Son bem Gilauben ser SGriffen follte man aber sod) ein Melyrers erwarten, als you je= nen Sermunftedluffen, seren Echroadbe wir fogleid fublen, mo woson wir zu viele ers: fobrung Gaben, onf fie unb in \&eisensftunben ijren Dienfi verfagen. Slber ber (B)laube, uno was er unb lebrt uns vergeif̈t uno giebt Dab - follten wir benten - würbe unb itt ber Seit ber serfuchung ood mutbig ex, byalten fosmen, ober, wenn wir mit unfrer

Edywad)= 
Eajmadkeit bes Granbens befannt fint sab werbe bod Diejenigen muthiger ere

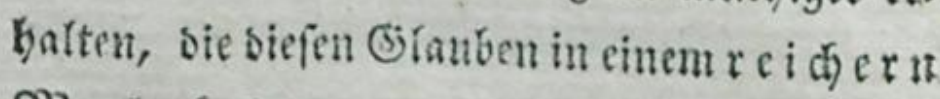

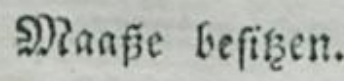

Es find aud von jefyer Sterbtiche gewes

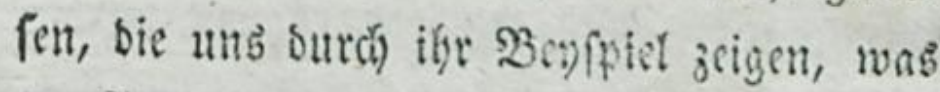
Der Fromme vermag, wem er fid jut innis gen Sereinigung mit (Sott binnngefd)wungen bat. -2 Inapb - Er fieht vor fid sment fhen, wie fie im Sefir unb Oenus ber SBelts freuben trobect, ben 2 (tmen verad) ten unb fich nllein fur bie Beglififten balten. Nber ifu liffern igre Frenten nicht, unb im \$efitis ser

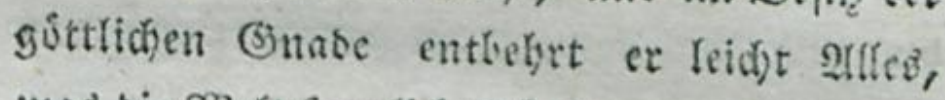
wab bie SBseft frerrlidjes bat:

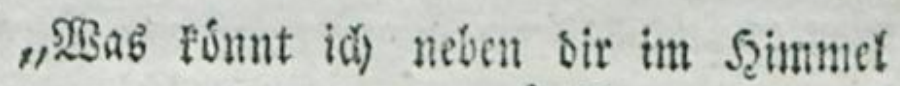
winifien,

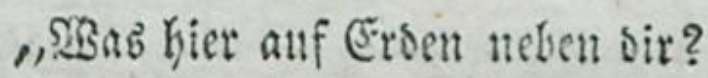

"Serichmadtet Leib und Sccle, fo iff Gott "Stetó meines Şerzens Iroft, mein Theil!

paulus war anç) zu biefer kogen Etufe menidlider Bolfommentyeiten gelangt. Das ber fount' er mit feinem Stanben 21 lles bubers

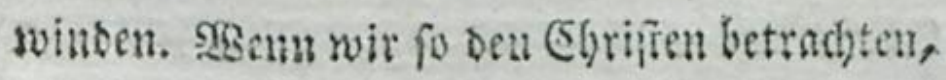

235

fiv 
fo bentent wir Yeicht, er muffé bey bem Shes wustfenn ber gôttlichen (s)nabe uno ifrer mila oen Einfliffe in feine Eeele - er miffe bey Den grofen Erwartungen, benen er entgegen fiebt, 2lltes obne heftigen Schmerz füblen, oulben, leiben uno ertragen fơmen. Shan reid)t ia fein (Staube gegen jebe 2rt von $23 i=$ Derwartigleiten (Brunde dar; zeigt thm im= mer, dafi bie Summe feiner S3ortheile, Ers wartungen und Şoffnungen unenblich grớzer fey, alb ber \$ुerluft, ben er leibet, voer bie Gefahr, bie ihm brobet. - Serliert er fein (3)ut, fo zeigt ber S5laube ifm beffen Eitelfei= ten und frichtigfeit, แno erimnert ifn, dafís er nod) unendich megr babe uno befize, als wab̉ er vertobr. - \&iegt er auf bem Siech= bette, fo troffet ibn bie 5 offimung ber 2 lufer= frefhung, woourch er cinen, nidgt ben gegens wårtigen Sd) badblyeiten unterworfenen, Gert= richerm \&eib erbalten foll. - Eterben ihm bie Eeinigen, fo weiß er, oa SBgelt leben, beren Freuben ungleid) gróser uns reiner fint

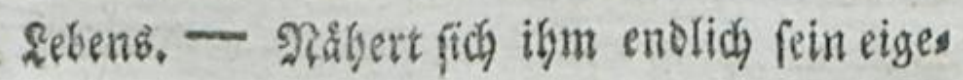




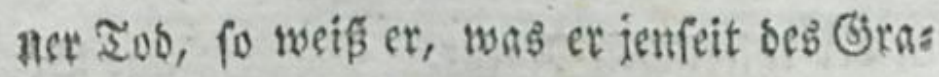
bes erwarten barf. -

„গুor Seinem 2Intlif̧ ber Freubent Futlle, ,21n Seiner Piechten Seltgłeit auf emig. $2(b e r$ wenn aud einige freilige, wenir 2rifaph uns paulus zu biejer bohen Stuffe Der Solffommentheit wirtlich gelangten, Daf irdifder \$ertuff, Edjmerz uno Gefabr fie nictst irren fomnte, fo gelangten fie buch gewif nicht glei (d) ju biefer bohen \$ollfommens beit. 'XBas mógen fie nicht erft gelitten uno

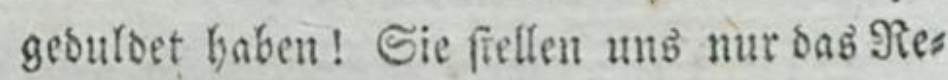
fultat ifgrez Sampfens bar, nicht ben Streit, bie Helung felbfit.

Der (3) Taube famn freylid einen פene fd)en zu ber Entfagumg feiter Selbft, zur IHfs opferung alfer feiner sortbeile, Entbegrung

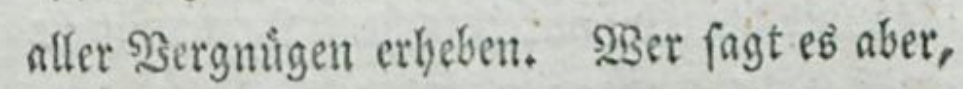

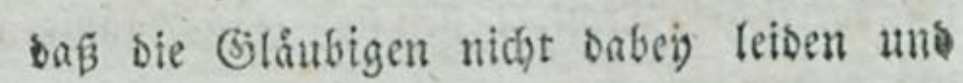
Sdymerz empfinden follten? -

Die Utractsen bes Gdymerzes, ber Erffarrung uno Betáubung ber Hnglicflichen uno ber anhaltenden Betríbni fiegen nidgt blof in feiner geifigen fondern in 


\section{8}

ill ser thierifden शratur. Sdmetz uns Bes tritbnifs if nidut a Ile in folge ber Ueber. regung uns ber Heberfbautung bes Unglicts uno ber baker zu befurditenden folgen. Sit SBevoes im bohen (Brnde, fo verewigt fich onb Uluglite uno madjt ifn volffommen elent. Edmerz ift Folge bes unmittelbaren Fin= studts, deit ein Utrfall auf unfer ganjes $\mathfrak{X B}_{\mathrm{B}}$ fen macht. Die eigentliche ?atur bcz @chmers ofo if eben fo unertalarbar, wie ansere

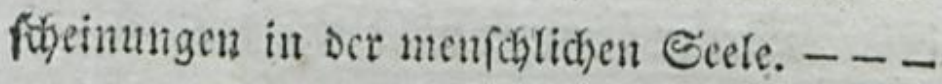

S tabelt baber bie 9) Jutter nicht ju febr, sie fich fo bey bem Iooe ibres (Gatten oder ifyes finbes beträgt. Bucifelt besiwegen nidit alt ifyer Sernumft oder Fefigion, wenn fie jezat Sende nidjt burt, fid ifrem Summer liber: lä̈t uno die Einfamteit fucht. Nerfobont fie nit soreiligem Iroft, deffen fie jezt nicht fábig iff; fommt, weinet mit ifr, befucht mit ifhr Die Srabfritte ifyer Geliebten; Gort jebe \&ub= rede geoulsig an, die fie ifren Entriffenen bailt. Serfudbt es nidyt surd) ङure ঐieden fie auf andere Begenfrande zu Ienfen uno ibre

Gie: 
Gebunfen yon ihren Seiben ariziellen zu wollen. Sie wirbe nur Eure Ireue bejweifeln uns Euth fiefyen. - Sann fie weinen - weinct mit ifr; fam fie beten - betet mit 5 byr;

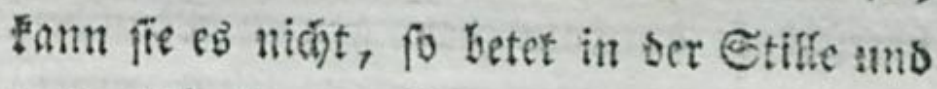
weinet fưr fie. - Erwatet yon Soben kerab Troft und Erfleidjterung für bie \$etribte, ba Şyt bod ihren Summer nicht fintorn fismt. Der \$ater ber NRenf(x)en, Dor unz nie sers Iapt, wirb bie Dulderimn nid)t Taffen; er weiro ctwas berfacteden, (oft eiu geringer alm[and)

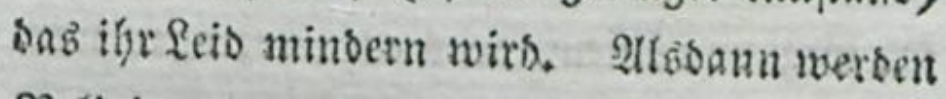
Religion uns 2 sermunft allmafitid tiber fie

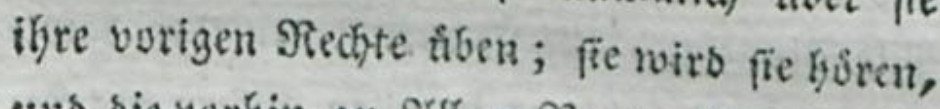

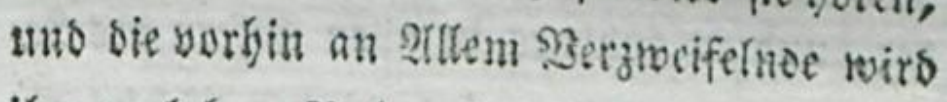

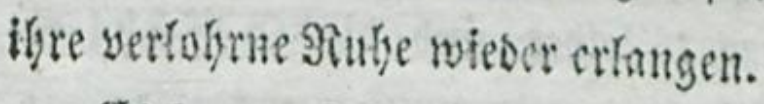

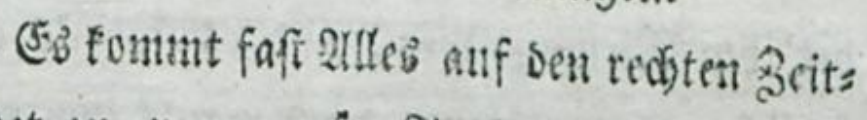
punct an, weth unfer Troft wirfen foll. Ebe und bevor eine \$etributif verbauet ift fagt Sterne — Fommt das Troffen Humer zu frith; und if fie verbautet, fomme's 3 แl fpat; zwifhen beysen (soranjen ift alfo eine faft brarfeime \&inie, oic cin Trúfter zu faffen

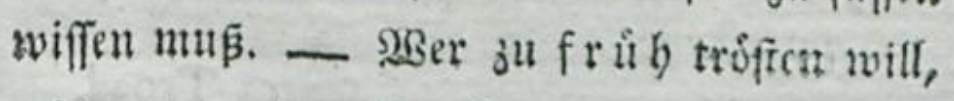
erbittert, uns verfiert Butrauen uns 2ultes.

ए5. 
ङE if unbegreiflid) unvernưnftig, cinett beftig feibenden in ber erfen 233 uth Dez Schmerzeb berubigen zu wollen Die ganze Sinnlidereit ift on anfgeregt und bes berrfdyt fo burdyaus bent ganzen Renfchen, bafs, fo lange fie in Spanmung ift, hiberles genbe sernunft burchaus nicht einbringent fa แก. *) - Smerfen Zuftand Des fürths terliden Reibens bat bas Şerz nur Gefühl fúr feinen Serfuft uno fúr foinen Schmerz, uno

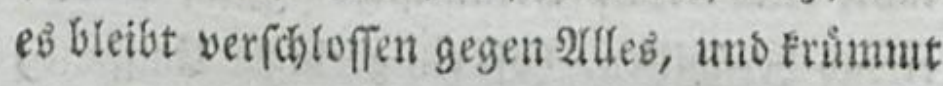

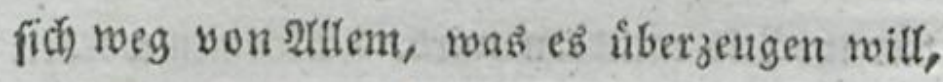
Daß̧ es weniger vertobr unb weniger leibet. Fllfe Sinne und alle Pierven reben ibm bawis ser, umb es fühtt es in allen Fafern bes \&es benb, was es leidet, tho dies (s) efúbl tes bet ifm Niemano weg, und eb finbet's ents fergfid) uno empireno, on $\bar{\beta}$ mau'b ifm mege

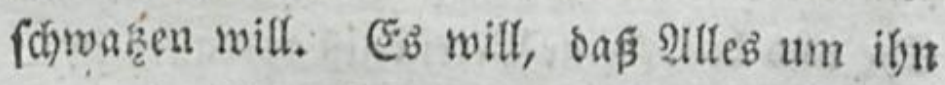
ber-mit ibm leioen foll - innigfe Theils แafgme uno gleidjes Gefübl für feinen \$ुers huft

*) Levis eft dolor, qui confilium capere poteft.

Seneca Troad. 


\section{1}

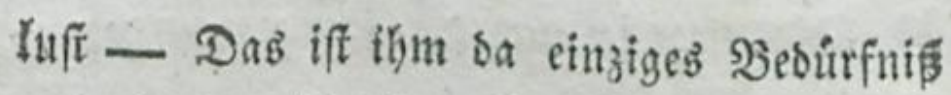
unt einziger Troft, alles 2 noeve vergrófert 3ur feinen Schmerz. Errf bann, wenn bie Menfchlyeit fich matt gefåmpft hat, wenn Der erffe Sturm aubgetobt uno eine gerviffe Stille forgt, wo sie Sinnlidereit ermattet fiegt, uno mur frille Iftrảnen nod) fliefen Danu refont fich oas feerz nad) Trofr, o a n n ervacht wahtres Seelenbeburtrnif nach Stâr: fung uno \$erubigung, wie fie \$ernunft uni Sibel giebt, uno ba nn erft if es biefes aros fies, biefer Stảrtung uns 3 erubigung ems pfánglich. *)

*) Ut medici in vehementi fluxu pituitae non ftatim fuccurrunt pharmacis, fed foris admovent, quod tempore concoquat humorem, ac tum medentur: ita in recenti dolore tacendum, donec tempore mitior admittat canfolationem.

plutarch in mor.

Ut medici vetant admoveri remedia, cum morbus in acceffu eft, ac faevit, fed cum fe remittit: ita ad primos il. los irae aut doloris motus non eft adhibenda confolatio, vel admonitio, fed ubi tempore coeperint effe leviores.

Seneca, 
Dies liegt in aud fo gans in ser गatur

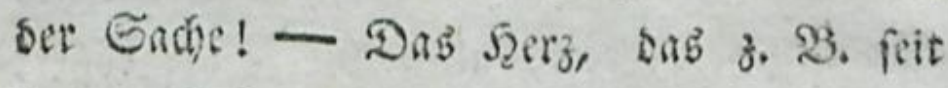

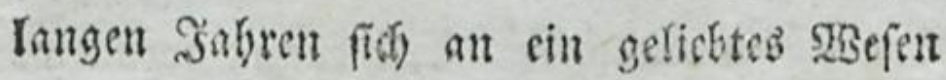
bing, bas fo gants in ifyn rebte, und fcit Jaghren bie reinfen, bep̧ten, füfeften \&ebengs frenben burch ifg empfing - Das foll fich mun bey feinem פerlufi gleiă faffen? foll ons entregrtide (Sefügl , mut mit Einemmat nicht „mehr zu befizen, was ifm fo lange bas bepste "Sinice bes Sebenz war" pogleid) rubig ors tragen fơmen? foll mit Einemmal feine feins fen reizbarfen ঐerven gewaltfam abgeriffent finglen, tmo mun plofictich Sorfellungen it feime sombe, blutenber ibe Seere aufulha men, and fich samic berubigen, mit benen eb fich yorber befto weniger vertraut ju mas den wagte, uns die igm eben bartma befits ungewohnter uns peinticher fins, ie mebse ifm dab Geliebte war? - Uno sice Berus bigungsgrúnoe ber rulyigen \$ermunft foll pith bab frerz mun gleich im wúthentefen Crum Der Seibenfhaft gewaitfam auforingen faffen, gegen bie noch feme gamze Menfobeit fich

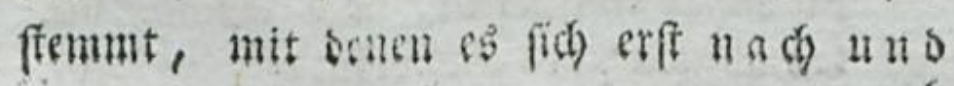
กณด 
na d) vettralt madien Eanti, unb benth es (iic) erf felbit freywillig entgegen oringen unb iffuen mup, wem ibre Sraft fich wirlfant an ibm beweifen foll? - S - uno went aud ber Sopf ba wol auffaft uns bie 2Batyrs beit refpectirt uno smimme - wabrliç,

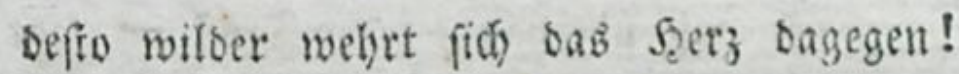

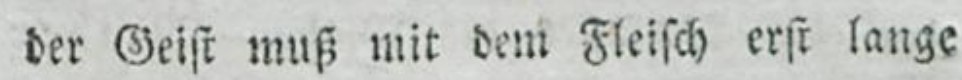
fimpfen, efy' er liber biefeb ben Sieg gewinnt, anto fo lange siefer Beitpunft nod nidst ba ift, if alleb Troffen umfonfr. De शaturt will

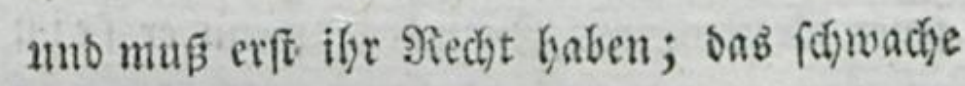
ångfilid) zแfammengeprefite Љerz will unb

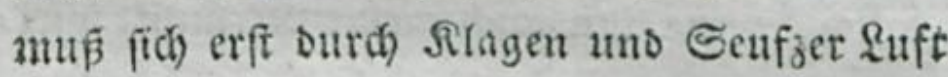
madjen; Der niebergebeugte (S)eif̃ will unt mus erft Beit baben, fich aus feiner erften ミetiubung zu fammeln und zu beben "), uns

*) Sermunft uno Glaube fins, fo lange es im Scerzen frurmt, unnirffom - fie fonnen o a nur magen, wo man bey rubiger Heberlegung auf Sorfiellungen meret; fonnen alsoam erft mit milbem 3ufpruch ben Edjmerz minbern, went Die máchtigere aeit es̉ in ser Esele rubis ger gemadgt bat.

(5)

Ђoppentitest. 


\section{4}

unb ber ift Meifter in Der grofen Runf, fith

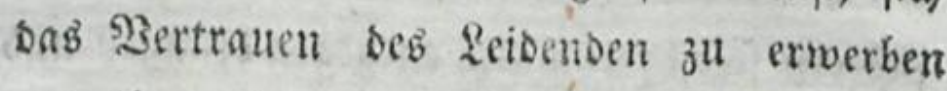
3mo thn 3uberubigen, oer nm erfien bas ouns fle (jew blt ber Traurigreit, moburch fein er quictender fichtfrabl des Trofé oringen fann, zu zerfire ue nerfiteht.

So wie tiberbaupt jeder Iroft - fagt ber eble Saffaffer bes $23 u c b$ : 23 eiber made ten ibu weiferuno glúctidin ben erften Ingen fdrecticher Ereigniffe an Dem leidenoen feerzen abgleitet, fo bielt aud jener Sruft meines శreunbeb, wem ex fei (Sefubl mit bem meinigen verglid), nid)t Stich, wemn ifn auch meine Dernunift wirts lid) annelsmbar gefunoen batte. (5) feblt oa geneiniglich an Ernfi, bie Mittel anzu= we noen, weidse fich zu unferer $\mathfrak{B}$ erubigung sarbieten. Dian verabichenet ba noch die

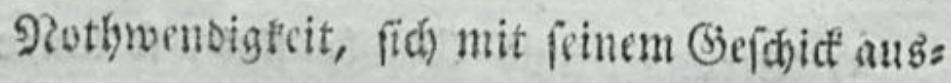
fobnen zแ mưffen, weill, wem iene כroft: gruinde auch) fonft ganj gefchicit finb, unfern Sufrand ertriglidger zu machen, ood) immer

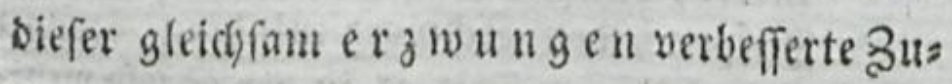
frano mit senem, we!dyen wir verlokren, feine

ßer: 
- Sgergleichung vertrågt, unb alpo nidit viel

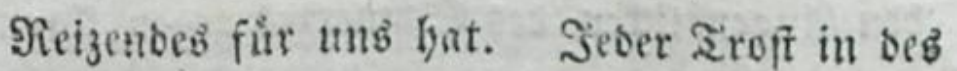
Ed)merzes crften Etunden uno ragen ift ouber nut immer ein aufgenotbigter Troft; meifiens ein unvolftummner Erjaks gegen Daś, mas wir verforen haben; bieb beftátige idb ourch) meine eigene (Srfalgrung. গoch mebr! So lieb mir die (jegenwart meis nes Freundes rwar; fo febr war er mir zus wiber, wenn er anfangen wollte, meinen Slict yon bem, woran meine Seele feft bing, abzuziefen. 2Aud meinen $\mathfrak{B e r l u f t}$ beflagen

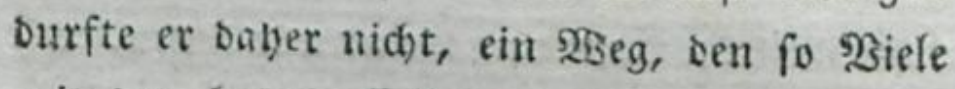
mit bem beften Erfolg einfd)lagen, wenn fie Dem leidenben 5̧erjen 3utrauen einfispen wots len, aber fur mid blieb er ofne গuken: benu ids fonnte mich immer oer unangenetss men Sorffellung nidbt erwebren, als wolle er gleidffom mein feerz daburd ju beftechen fuchen. Nur feine theilnelymenoe fiflle Thrảne war $\mathfrak{S a l f a m ~ f u r r ~ m e i n ~ f r a n t e s ~} \mathfrak{f}_{2} \mathrm{er}_{3}$. - " 8.

SBillf ou baker ein wohlthátiger Tros= fter Des Reisenden, Des Ingludilicjen, bes (5) Siran= 


\section{0}

Siranten und S3etzagten feyn, fo rebe nidit in Den 2 tugenblifen Deß Schmerzez gegen ifren Fummer, tabele nidgt ilgre Seufjer, migbils Yige nidgt ifre Silagen, veroamme nidbt ifre Thrånen, fonbern chre ibren Edmer3, fey foroneno gegen ifre Elagen uno beweife ibnen die zintlid)fe Cheilnabs me an ifrem Edomerz tho an ifrem

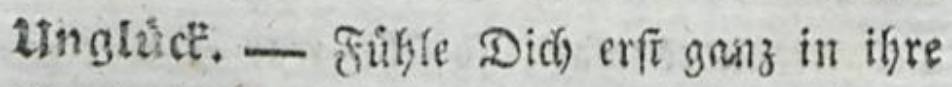

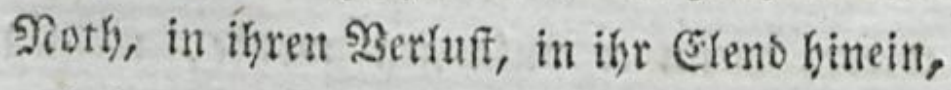
unb baun gef' zu ifhen mit theifnefymenten

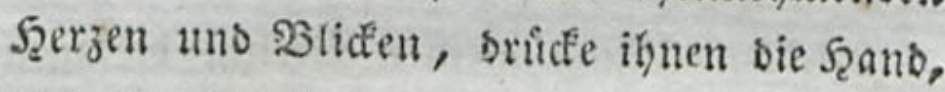
unb traure, flage uno weine mit ifnen, wie

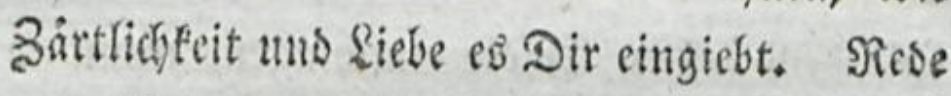
mit ignen son ifrem S3erluft, yon iffent Sd)merz, von ihren jerfistien Szoffnungen, yon ihren traurigen 2lubifidten - warme, Geiffe SGrinen loctt Dab nus ihren 2fugen, aber glaul nicht, sop su basurch ifren Sd)mets yermehrt haft - o folde Thránen zu weinen, erteidgtert fie unaแsfpredglich uns find ifnen sie füpefie Sigohlthat! *) Unters

*) Fletus aerumnas laevat.

brich

Seneca 'Troad.

Fle- 


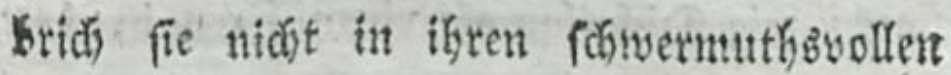

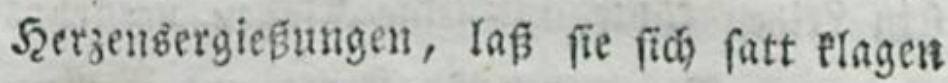
unb fatt weinen, uno grlles, was fie cunnmert uno nagt, yor Dir ausfétitten, tho zeige

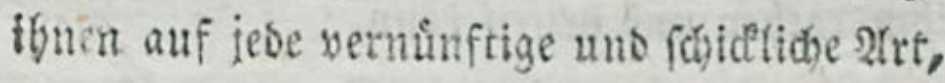
wie fehr Dut ihr Eeioen fublft, wie berglid Du fie bebaterff, uno wie gern uno gervis Du 2lles thun wirft, iffen Sunmer zu lins Dern uno ifgre feioenslaft ihnen zu crieichtern. Dab wiro ifyr ganjeg $\mathcal{S}_{2} e r_{3}$ औh Dir neigen, mit volfer auverficht gegen Did erfüllen, fie vorbereiten auf Deine Tróffungen uno Did fábig madjen, ibnen झlles zu feyn unb zแ werben, was Du ifnen feyn uno werben migteft. -

Srite Didh aber ia yor aller Empfinbes Yey, yor allem Edyein ber 2fffectation, uns yor ber geringfen Hebertreibung in ben Bes weifen Deiner Theifnafme; fey jâttlid uns warm gegen ifh, aber auç månntiç uno (5) 3 frarf;

Fleque meos cafus: eft quaedam flere voluptas,

Expletur lacrymis, egeriturque dolor.

ovid, libr, trift. 4,3 . 
ftart; ;eig Dich ifm als cinen frreunb, bee fein Reiben filfit und feine Silagen verfiegt,

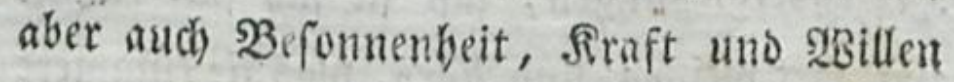
bat, ihm zut rathen uno zu helfen, wo er ihm rathen und belfen fam.

\section{7.}

Dann erff, wenn die Spannung dę erfen Sdhmerzes nadigefoffen, wenn fid bnz Serz aub Dem beftigfen Eturm beratrigear. beitet bat unb eb in ber sunfeln Gewitter: nacht wieber ju dimmern beginnt - sam, in folchen rubtgetn 2lugenblicten, Pồ te fie surd Croffgrinde der Dewnunft uno 3ibel, die für ibs ber'3 uno fưr ibre Lage paffen.

Sind fie sou bem ङalage, ber fie traf,

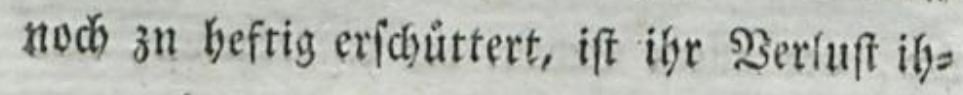
nen nod) zul nell uno zu gegenwortig, tobt's

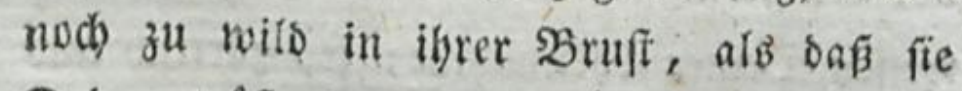
Deinen troffenben uns belehrenben unterridgt faffen, tiberbenten uno fich zueignen formen - Trab foll Dam all Dein Trófren? -

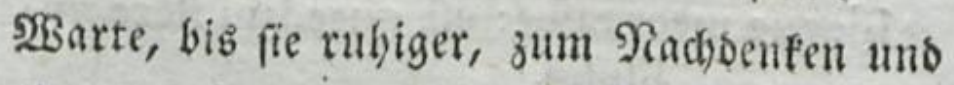
Emp finden uno 2 eberzigen Deiner truffenden

ISBhy 


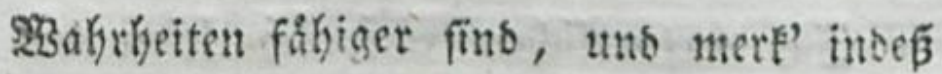
Darauf, son welcher Ëeite Du ifhnen am beşten. beyfontmen uno wosurch Dut am fráftigftent

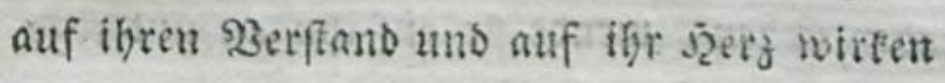
E̊onnefr. -

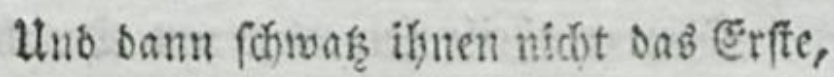
Befste vor, was Dir cinfällt, fonbern fag' iknen nur Dab, wab in ibre Dentungss. uno Empfindungeart eingreift, was ifyrer ief̧igen Enge, iffen iefig gen Beburfnifs fen, if rem 2 lter uno if ren Berbinbuns gen am angemefienfent if, tuno wab fie ver fteben und fi dh o theignen formen.

Du Gstft, indem Du Did mit ifnen unterrebeft, dos biefer ober iener Umftanto bey) ifren Eciten ifuen ben meiffen fummer mad)t: bente nad), ob Du nid)t einen furjen fråftigen Sprud atı ber $\mathfrak{B}$ ibel, woer einen rúbrenten Gefang vober \$Bers, ober ente riths

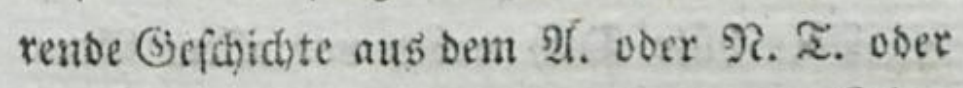
ein SBeyfieiel aus Deinem voer ibrem Seben

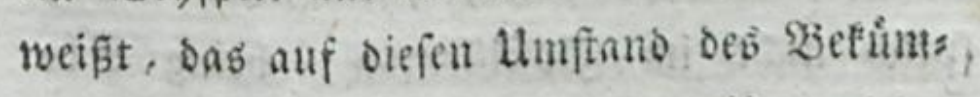
merten past, uno worats or Hnterridst, Troft und Szoffinung faspfen tamn.

$$
\text { ह } 4
$$
ริ่า 


\section{0}

Sim yettraulichen (seiprad reitet ber Leibenbe Dich, woer Du leiteft ibu, auf siefe ober iene trofivolle Stelle ber $\mathfrak{B i b e l}$ - bleib babey fehen, wende fie auf ibn an und rege mit SBarame fein Gefungl bafir alf.

Doer er theilt Dir biefe uno Du theifft ifm jene mertwurdige Erfabrung Eures Q

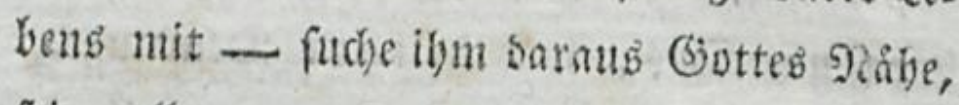
feine allergenauefte 2 Uufficht und \$aterleitung beutlida uno wichtig zu madjen, uno reite baralts Iroft uno Soffuming fir ihn her.

Suche ihm bann hiberfhmpt die Roths

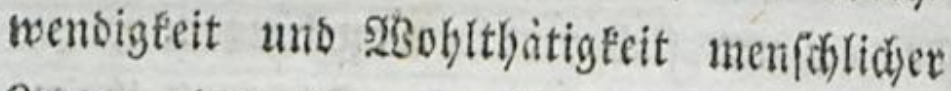
Seioen, Die weifen uno gutigen श(Gichten, sie (Gott nuth mit i hm babery erreichen will, sie \$3erfichrungen Seiner gôttlichen Unterfithąung und Sertlfe, uno bie nabe unzertrennliche \$ers binoung biefeß \&ebens mit oer Errigfeit deuts lich und lebhaft darzuftellen.

2tber bute Didf babey vor allem भres sigen; beclamire nidht; baranguire nid)t; farich niơt im S3offillenton; bring 20 ba we d) \& lung in Deine Huterfaltung; merf barauf, welche Sorftellung am tieffen auf ikn 


\section{$4 \mathrm{r}$}

ifft wirte, tum fie gelegentfict iffu bon ciner anbern Eeite wieber vorb 2tuge zu ftellen, aber bafpeie feinen Beomfen ins Unenoriche; Eriç) oft $a b$, wenn Du bemerffr, onf̧ eine

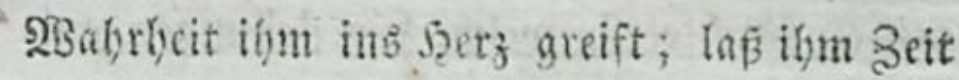
fie ganz ju befendfen uno ganj zu Durchfobauen; Gore feine Eimmendungen - waren's auda Die ffarfen Sophiftereyon - mit Emftmuth an, uno beantworte fie mit Siebe uno Rube; geh' auf mocre (jegenfínbe mit Reid)tigfeit ñber, fobarb er Das ju wuind den focint, uns Ino Dif uberlgaupt mebr yon ibu leiten, als onf Du ibn leiten wolltefi.

Srandelft Du fo mit meifer 2Ufmerffam: feit unb fonfter \&icbe, fo wirft Du gervis Deine menfdicheole 2(bficht nicht verfenten,

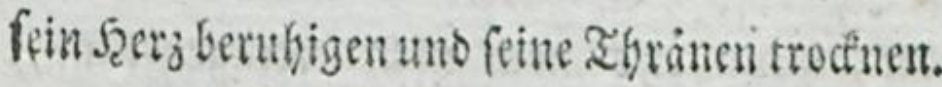

8.

SBillf Du Dir bals uno volles 2 sers fraten ocs Lcibenden erwerben, fo fuche

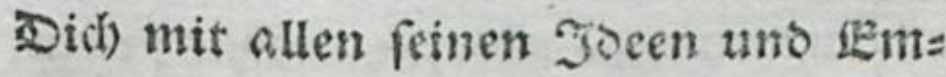
pindungen fo vertraut als mogtid 3u madoen. - Seidjne ím bann felbft sen Gang feiner Empfindungen vor, fring mit

(5) 5

Deis 
Deiner Senfonentenntnis tief in fein Sers binein - befareib'b ibm, wie Dir in álgn= richen Fallen zu \$nuthe war ober foyn muirbe, was Didh am tieffen fohnerste, wab Du am meiffen fürchteteft, welche Sorffellungen am rangften uns lebraftefren in Deiner Ceele Gafteten 2 . furz fprich) ihy alle feine (ems pinoungen uno \$orffellungen aus feiner Eete beratts uno laf fie ilgn bey Dir ganz wieser finben. -

Saz ihm bant, allf welchen 2 Ssege fich nadber Dein Schtrerz allmálid) vertohr, was aufferoroentich oaju wirfte uno worim fich Dein herez feibft balf, uno wie Du jef̧t Deine ganze bamalige lage anb einem ganz anbern

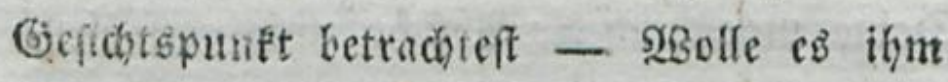
aber, wemn er's nod) nicht ertragen faun, nun burdaus nicht eimreben, baj er nut grabe biefelben Erfalyrungen maden merbe. Deine Errzåhlung if fchon Zrofi's genug für ign, uno um befio mebr, je wentger fie den Schcin bat, als wolleft Du ilgn grabezu bas mit troffen. Ein Troft ber incognito fommt, ber gar niōgt die Mine bat, tróften zu wots

ten, 
Yent, wirft unenolid methe, als alle Bernthis gungeggrimbe, bie man bem feidenden gemalte fam auforingt, -

Du wirf ibu auf bicfe 2 Beife feft an Did) zirfen; yoll ocs inniaften 3utrauens wird er Dir fich bingeben, Feine feitier Em. pfinsungen Dir verhellen, unb gern unt wits lig jeben Rath und jeben Troft yon Dir ans nefmen, weil Du ifn basurch liberzengt

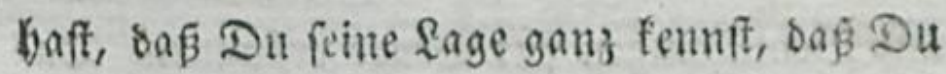
vollig competenter Beurtheifer foiner bies muttlfftimumung, und grabe ber freund biff, ber am Kerzlidffen an feiner Lage Theil nimmt, unb am wirfanfen ihn mit Rath

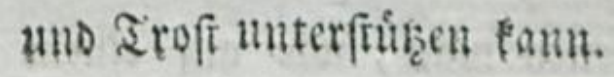

$$
9 .
$$

Eoll ber Reibenbe Dir ganz trauten unb Dein Troft auf ihn wirfen, fo berveip (Did)

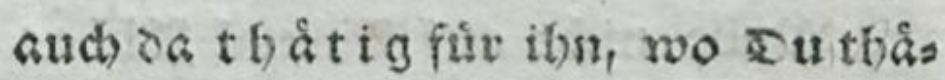
tig für ibn feyn fa $\operatorname{lu} n$ ff. - Iber ba frecl't's! Selten wollen ong bie Menfden, uno barum gibt's fo viefe leidige Trôffer!

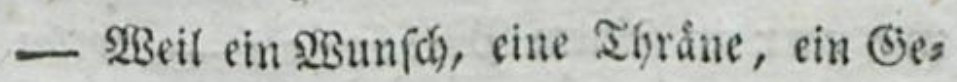

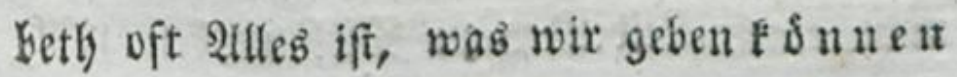
ragt 
44

- foigt $2 \mathfrak{b} b t$ irgenswo - po twits ed stoch ifter Alles, was wir geben wollen.

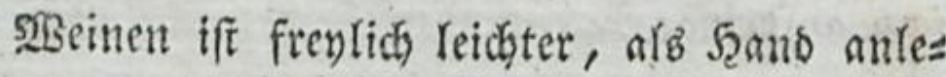

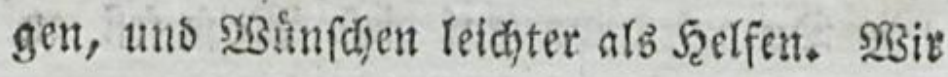

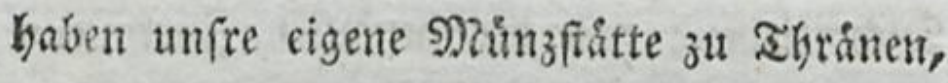
aber niç) zum Gerbe, uno unfre Senfzer fü

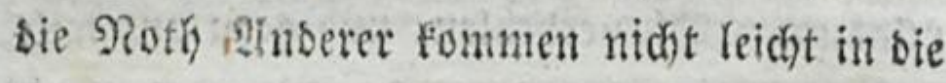
Sueere mit ben Seufzern fur unfre eigene, aber wobl fosmte es fich bey unfern Bangent 1mb Bemukhungen fúr Seende cráugnen Das ber begnigen fich fo siele, ihe gutes $\mathfrak{F}_{\text {erz }}$ Viever burd) naffe 2fugen anz"lzcigen, als burd

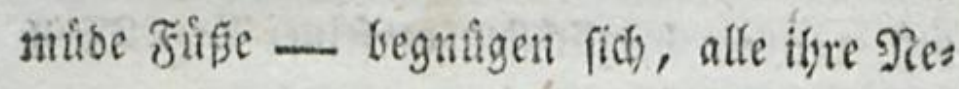
benmenfacen wie abgefchicbene Seelen zu bes tradten, ber benen man, wie auf einent Gottešncere, mit einem berslicten guten Ges banfen abiommt.

\section{Io.}

Wolle nidtst auf leimmal 2fIteo wirten mit Deinen Trófirungen, uno bite Did vor Gefhwaisigfeit. Shan verfehlt feinen siwed - fagt fit ie me yer in feinem gुg ilotab - wenn man einem Retbenden ju Niel werben will. গ̧iel 


\section{5}

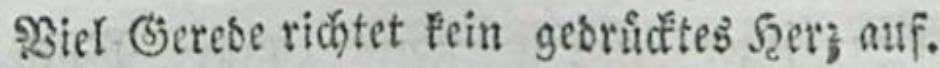
Tiefunt nach und nach bald bier bald surt

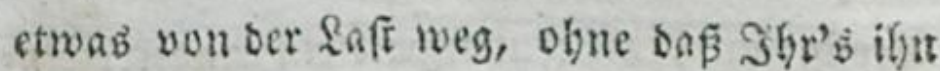

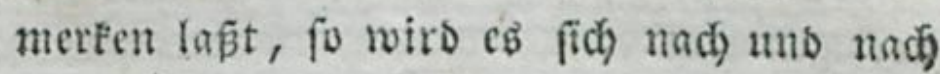
wieber giben fónnen.

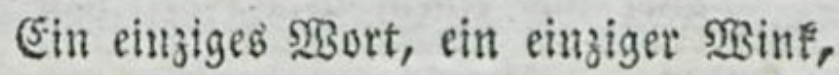
out rechter acit gegeben, wirkt oft unenolián mebr, alb alle Sinnfe ber Beredfamteit. Man teitet sasurch oft, olyne oen Schein zu baben ę z̆l wollen, ben Slicé bez̉ \&eibenden auf 2 Bablyeiten uno fúbrt ibn unerwartes

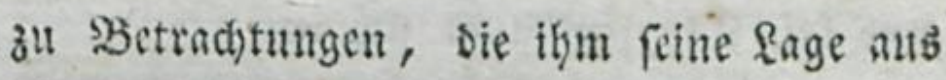
cinem ganz anbern Eefichtöpunft zeigen, uno

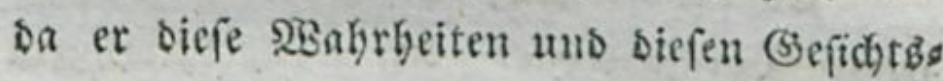
punet felo fi findet, wenigfenb felb f gea funden zu baben gla ubt, fo geben fiefornell in ihm mit voller Rraft zur Lleberzengung ůber; $\mathfrak{e b}$ beginnt ify ein Ridjtftrabl in sie Seele zu dammern, er fublt arieb in fid, Dem wohlthátigen Strabl weiter nachzuges ben, und fiebe! - indent er gebt uns forfact, finbet er einen neuten 23 abrbeitรquell, ber bell und flar yor ibm auffprubelt tunb ber ifym erquicfende Labung reidgt - uno fo gab ihm 


\section{6}

finm iener einzige SBsine melye Ruth,

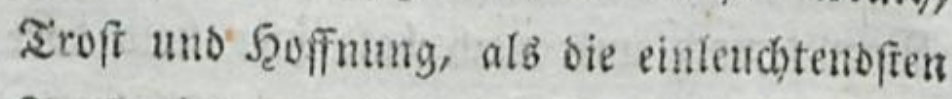
Troftgrůnbe, oie iiju irgenb ein 2fnderer ein= predigen wollte.

$2 B e r$ mit einem 3 Hahregen yon Trofts grimoen - fenen lie tibrigens aud noch fo verninftig uns gut - úber einen feidenden fällt, ber wirft grabe fo, wie - ein ЭJ[aţa

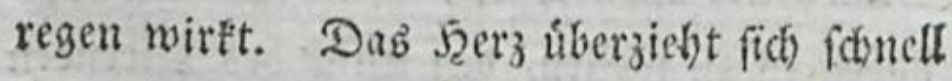
mit einer fefren, unburchoringliçen ßinbe, und fein Tropfen fautgt fich ein.

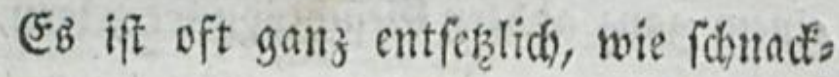
felige Sুbtrafentriffer ben Niebergebeugten mit ibren zufammengefneteten Sentenzen mifbanbeln uno alfs unbarmbersigfre mars

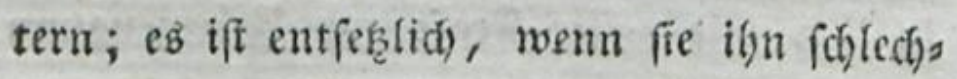
terbings nidbt zu SBort fommen laffen wolo ren, wenn er wie ein Silof fich yon ifgnen behandelt fehen mus, wenn or feinen \&aut und feine Slage fich erfauben barf, uno wenn fie meynen, bał alles leid ein Ende babe, fobats jie nur iffen tunerméstidgen Enct soll erbaulidgen (S)etrátfóes uno alteluger $233 e i b 3$ beit 
heit ůber ihn her(屯hitten. *) - Die Seute formen gut ratben - fagt Leonato bey Ebafefpear - unb oen Edmerz troffen, weldyen fie ferbft nidgt füblent. Siber fobald fie felbft in umpern Fall fommen, fo vermanbert fich ihre SBeibheit in Reibenf(baft,

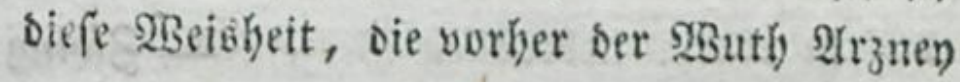
verforetben, bie frarte Paferey mit einem feibnen Jaben feffeln, ben ভd)merz mit $\Omega_{u f t}$, anb Tobeşqualen mit 230 orte bejaubern wolfs te. Nein, nein - bem lunglitcllict)en, ber fich) unter ber Laft feineb (Elenob frummi, Bedulo zufprechen, bas Fann Sedermann; aber to weit erfirecft fíc) feines Menfchen Zugeno noch 2 setsheit, baß er noch cben fo moralifón fenn founte, wenn er felbft ein

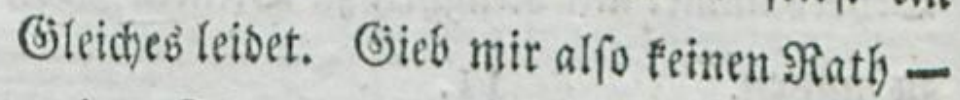
meine Ed)merzen (d)renen lauter, alb alle Sorfellungen.

S baft Du mahres Nitleiben mit bem Inglictliden, und liegt Dir's mirtlich an, fein

*) Les confolations indiscrettes ne font qu'aigrir les inolentes afflictions.

$$
\text { Roulfean. }
$$




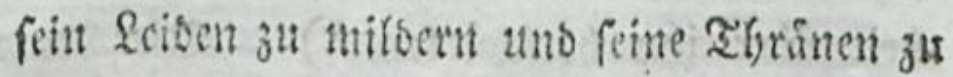
trodinen, fo wirç Du nicht glauben, Das thm mit (3) efd w a geholen ift. Raplieber Den Reibenten resen, wenn c: teben fann, und gegen Didh reden mag; fuche jeber femer Enpfintulgen \&uft zu machen unb faffe fie auf in einen livbevolten Sergen. - Siwing ihn aber audb nidjt zll teden, wo er nicht fam ooer will, uno vergíp es thberbaupt nie,

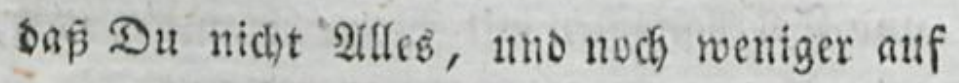
Einmal ouf thn wirten fannft. Der $3 \mathrm{e}$ it uno ben thmfảnben, bie fic) in in jebem 2us genblict verånbern, muf ein grofer Theil uberlaffen weroen; wenoe baher auf biefe ins mer Deine grosiste 2ufufmetffamteit, uns fudye Bendes inmer mit Sirtugheit ju Deinem Buect วิน แนหุen. II.

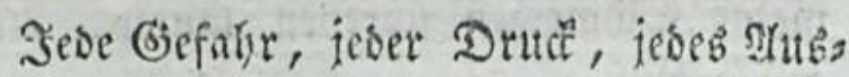
Gieiben ber Shutfe fómebt dem Iribfinnigen

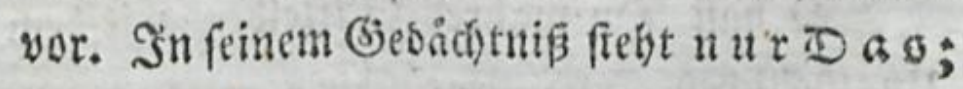
peine Einbiloungblafaft ficht $\mathfrak{n} u \mathfrak{D a s}$, ats bab' er nie etwas 2 tnberb erlebt. Frlfe Şeweife von Şülfe Gottes, yon Entwiçetung unfers Schiffals burd Shn; alle Erfahrums 
gen von Erbjơrung bes Eebethb, sie oramas tifoffen Umfdomulinge im leben fins gefdhwun= Den, wie bie Sachtberveife Je fu s gefhrouns ben waren aub $\mathbb{C b}$ omas Sinn. SBeil ę̉ nid)t grabe auf bie 2 rtt geht, wie $w$ ir Denten; weil nidet geholfen wiro zu ber Seit, ba wir'b erwarten: fo gibt's feine Şulfe, fo ift Alles verlobren; die beftimmtes ften, unwegerfläbarften SBorte Sot tes unb Jerus finb nidt gefagt - Jefus

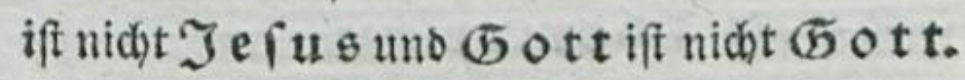
Dan fann nid)tb beffers thun, alb fterben; man will frerben - wünfdt fich ben నob. G)ernbe, alb ob man feinen Sinn, fein $\mathfrak{S}_{2} \mathrm{erz}$ nidbt mit bimbernábme in iene $2 B e l t$ ! (5es rabe, als ob bort nidbt aud 2(lle Beit zur Reife baben muiste fo gut wie bier!

Im meiften f(blägt's nieder, wenn man anbere gute, glaubige 5 o t tes uno Jes fus જerebrer ob ue frulfe fieht; went fie bem Ed)ein nach vergeben bitten; wenn

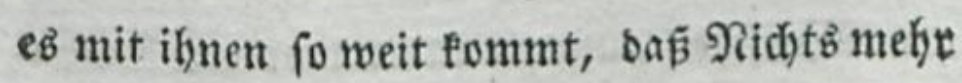
zu boffen ift. "So wirb's aud D ir geben! "fo vergebens mirft aud D D boffen! $2 B a B$ balf 
"Walf senn alles bas gitten uns Fleber, uno ".2Barten uno Giauben? Gott bifft, bort, "erbort nicht mebr! গicbtb Seffers alb ber "Tod!" - Das ifi bann bie Eprache des Rleinglaubens, oer 9utblofigleit, und fie if grabe fo vermunftig und fo thorigt, wie bie Eprache des $\mathrm{Cl}$ om a o war. Sech Tage bernach) zeigte fichs, wie vernimftig er geurs theilt batte; uno freylich, $2 B e r$ bie Beit ber Entwidefung a b warten Fonnte, ber wåre weifer, alb $\mathbb{C}$ bomas war.

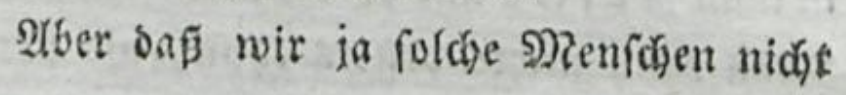
rid)ten und verbammen! dẩ Dab nur ia Seinem einfalle, oer nicht leidet, nie tief ge= litten bat! Immer folwebe fie unz vor bie Radjpicht, bie fanft oulbenoe 2trt, mit oer Jefus feinen Tbomas befranoelte.

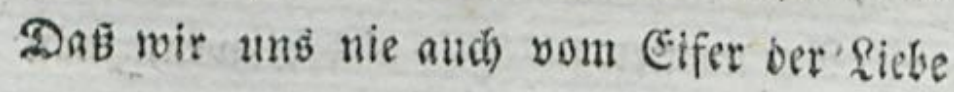
biutreiffen laffen, foldyen Rleingläubigen fern (5) lauben a thoringen zu wollen! 2Beder wabrer noch falfober (b) laube if ie cis nem SRenfchen nufgezroungen worben, $f$ a і5) ie sufgezwungon werben. Beng' ifju you Deinem Grauben on şabrheit, Treute 
Into Seiffe Botteb; nimm rgeil an feinem Seibet ; füble Dith in feine Sd)wádse, it feinen నrubfinn, in peinen Rleinglauben bins

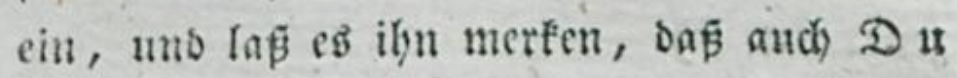
folchen. Sinn für bas grifate Leiben båtrtit; yergegennartige ibm in einer guten Stunde Den (S) suungôfofen, fur Den a u d) feine Stulfe zu feyn fúfien; wolle bayon feine sBirkung ets

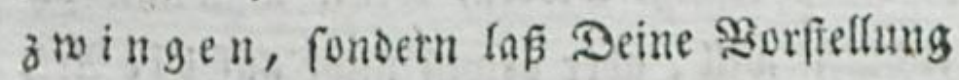
wirten, was fie wirten fann. Seibef $\mathfrak{D}$ felbft: To zeige Deinem mitlcibenben Bruber

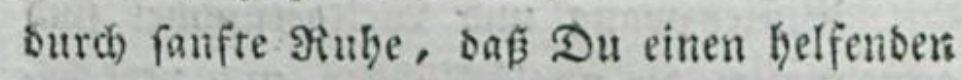

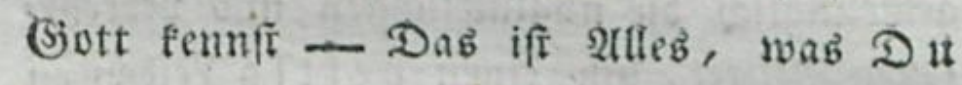
thun fannft: Sott bat fid's yorbebalten, sem tiefleibenben, jweifelnden, verzweifelnben

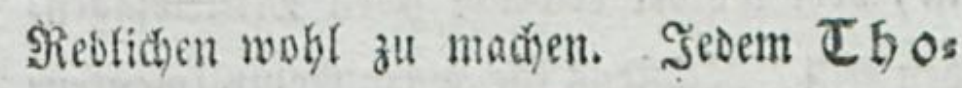
$m$ as fonmt fider eine Stunbe, wo er, bes

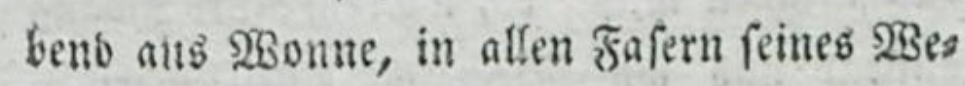

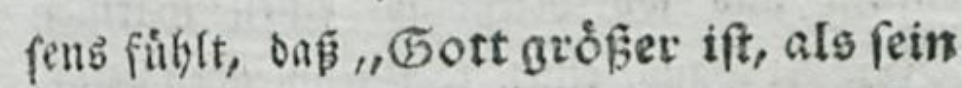
berz;" wo er Durds alle fúnf Simne úbers zeugt wirb yon ber $2 B a b r b e i t$ Deffen, wab ex nidgt glauben fonnte, uns betåubt yon 2(llem, wab ef erfăbrt, nichts 2(nders ftamp

D 2 melts 
meIn faut, als: "mein berr uno mein Gott!" 2(ber bann glaubt er gewis aud) feft, and lebt und firtbt Dem, ber ibn fo úbers zelugt Gat. *)

\section{I2.}

"Suthe sen Leidenden 3u zer: frreuen!" Das if ser erffe Grunbfá, nact) Dem die Freunbe bes \&eibendén arbeiten; uno er mu

- rubigen will; aber gewís nicht auf o ie $2 \mathfrak{t r t}$ wie ihn oas unweife Tróftervolf befolgt.

Bip̄tenzroang und SBeltgetútmmel fint gemeiniglich bie unglúclictfen uns verfebra teften Nittel, um cinet beftig \&eibenben วu jerftreuen. Da, wo צtlles beiter uno froh

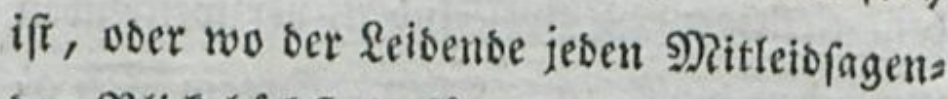

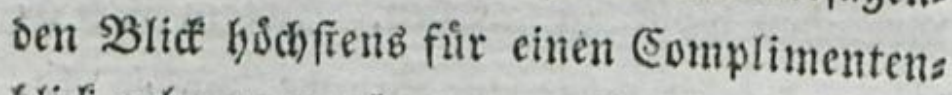

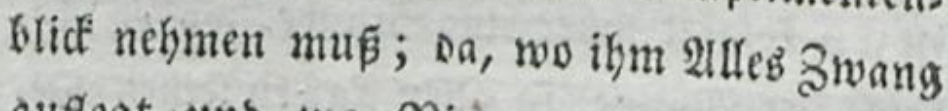
auflegt uns to Niemans einen warmen

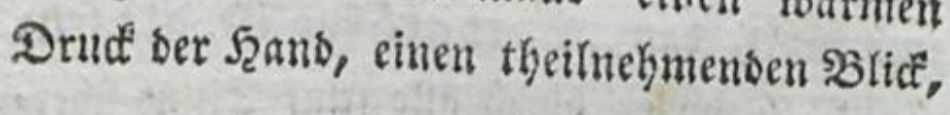

fein

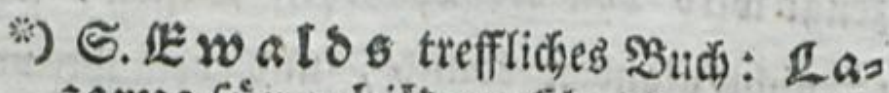
3 artis fuir gebiloete (briftusvereh= rer, befonders firt Leidende. $\boldsymbol{Z}_{\mathrm{er}}$ Iin 1790. 
ein herglides 28 ort fur ihn bat; wo man ifhn bidgften Ealt betautert uno গiemand bie Fulle feines Edmerzeb fennt, Niemand bie Duaal

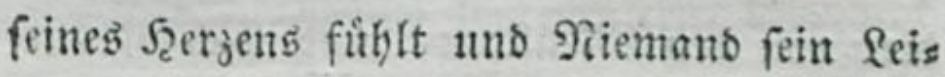
ben ifh tragen bifft; wo fein funnterfolwes reb $\mathcal{S C r}_{2}$ tun fethe troftlofe Seele fich weg= wentet yon jebem 2trbfict und yon jebem (5ies แแB, Den man ihm auforingen will - ach! babin taugt fein Reibenber, ba finbet er wabre lich) bie wenigfte Berfireuung, uno ba vergifit er anch) fein Reiben am allerwenigften. (5r allein fiel)t fid ba unglideflich) und einf(im une ter all ben Mienfthen, uno oie einzigen (b)e fuible, benen er fid úberlaffen mag, will man ifm ba rauben, unb barum muf er fie tief in fein Snmerfes verfaltepenen, unb diefer Swang emport ify uno verooppelt feinen Ed)merz uno madjt ifn für seib uno Scele jermalmend, **)

\section{3}

Se:

3*) Crefcit diffimulatione ipfa dolor, hoc altius demiffus quo minus profiter1licet. g̈ufin. 1. 8.

Strangulat inclufus dolor atque cor aeftuat intus

Cogitur et vires multiplicare fuas. Ovid. Trift, V. 


\section{4}

Betrúbte, bie ben Ios einer geliebten 93erfon berweinen - fagt 3immermann*) -

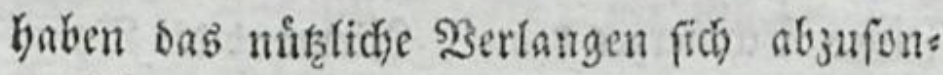
bern vou ben Renfden, unb bod) arveitet man Demfetben yon allen Eeiten entgegen. Man will nid)t, bnf man Itaurigen yon tfrem Berluff fprectye. Sieber umgicbt man fie mit einem Edjwarm gleidgufltiger uno

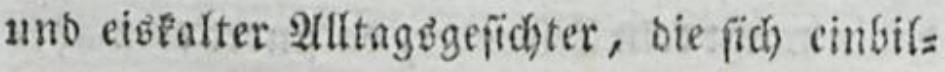
Sen, man miffe Traurigen nur Sifiten mas den, und fie vom Morgen bis in bie Ninche

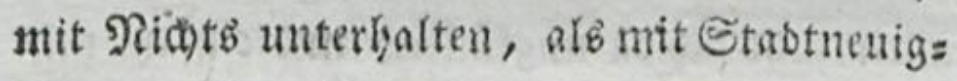
Feiten; ifre Traurigfeit werbe fich bann

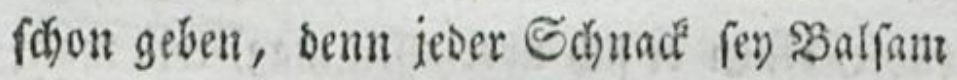

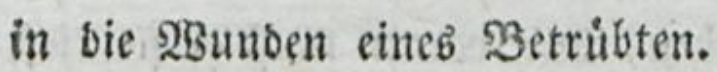

Lafst mid alleine! bact)t id taufentmal, alb idy to reniger alb zwen Sanfren nach meiner 2Infunft in Deutfohlant bie innigfi gcliebte Giefåhrtinn meines \&ebent verfohr ac

Gey oaber weife uno vorfidtig in ser wabl der ntittel, woourds bu den Leioenden 3 setfereuen fubff, went

") Ueber sie 佂infompeit. 3ter నh. 
ment bu ifyn- nidjt quafen uns pein Seiben nicht vergribern willf.

Naturgenu1, ") Naiti, *\#) zwectmâs Eige Rectire (movon aber mur felten (S) brauc) D 4 gemadit

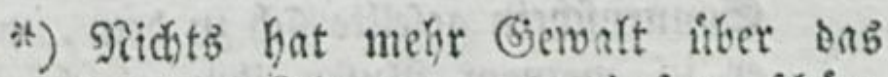

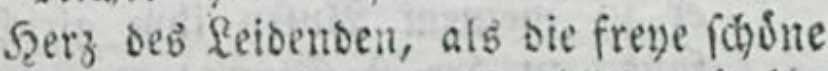
Natur; é ift unbefdreiblid) wie ihe Genuี bie Seele erveidyt uno alle

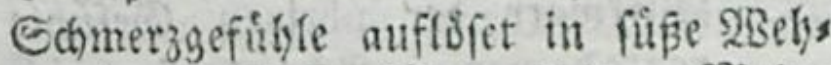
muth uno fanfte Mefandulie. Meine Bråfte waren fehr erfospft - forribt Der Jere yon zi o 5 e bue (in feimer

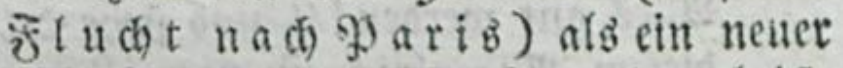
Błutfurur ben gewiffen Too peiner heifs geltebten (Battiun ibm anetunbigte. Die Nergenfonte befdien Den (Etfer mir gegeníber; bie Ruft war warm,

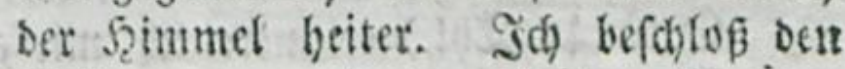
Sd)lummer meiner geliebten (Battim 3แ nแsen uno ein wenig binatts ing

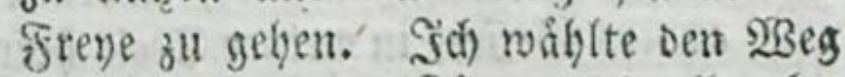
nad) Belvedere. Rounte id alles bes f(c)reibu, was id) nuf biefem 23 ege ges Dad)t, empftuten, gebetet, gefurdotet

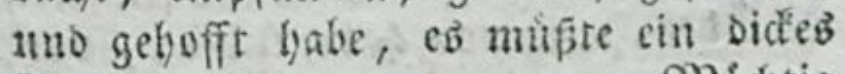
Bud meroen. _ _.._- MRidstig ift oer Rei; our Nentur! Selfft an bies fen forectlid $n$ Sage wirtte ihr Zamber ouf meinte Enn, uno fh.ĥferte enolich meine 2(ng|t eis. Der beitre warme Son' 
gemadit werden fann) uns - yor allem

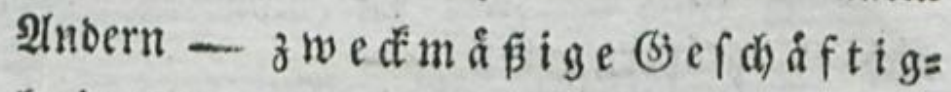
te it fino die beften Mittel ihn зu zerfireuen und zu berulbigen.

Itnter

Sonnenfchein gefellte fids zu bem, was er in meinem $\mathfrak{B u f u n}$ ibm gleidiartig fano, zu oer รૃoffnung! er entfaltete fie gleichfam und jie erbob fich liber oen SBirrwarr ber librigen (Sefúble uno ragte bervor. ,2I(d)! - fagte id) plosh= lid) laut - mir war als ob id) la ut fprechen måste - "es fann nods 2rllez gut merden!" uno fielye oa, vou biefem 2lugenblite an vermaltete meine. Şbantafie ibr mobltbatiges 2(nt. -

Mit Shrdnen Danfovoller Rubrung fibreibt S̈mmermann - Neber ote Eimf. tr $\tau$ bl.) erinnere id mir einen rag aub jenen erften Beiten meines 2lufentlyalt in Jeannover, in benen fein anders (sefubl in meiner Secle herrichte alb oie fchwarzefte Scelanfolie uno $\mathrm{dab}$ furchterlichfte Jecimweh. Job fam in Den fleinen (5arten meines fel. Sreundes, beb Jeerrnvon binu bev, vor fean:

- nover, uno weg war für diefen Iag mein Seimweh. - Der בauber war mir neu. গod) wust' id) nicht, oas fich auf einer ganj fleinen Flikbe oie श्रatur in folder reizenoen פ) inmidgfaltigteit 
Unter allen Nitteln zur Betukigung fagt ber eole in feiben aller 21 rt eingeweifhete serfaffer des treffliden, jebem \&eidendek zun jedem Frumbe ber feibenden angelegents

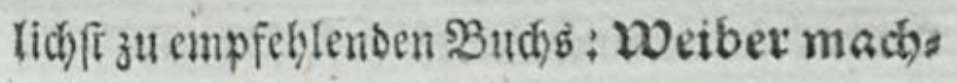

\section{5}

tert

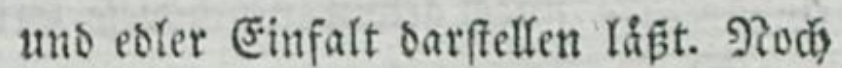
batt' tă nicbt erfabren, oaßs man auf ben eriten 23 lid fo weggeriffen werden faut yon 2llem, was uns in ber Etabt

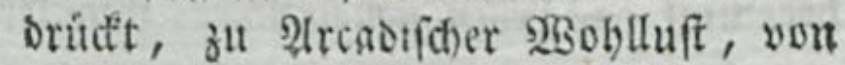
allen Gefüblen, Die uns das \&eben wies ber froh madjen.

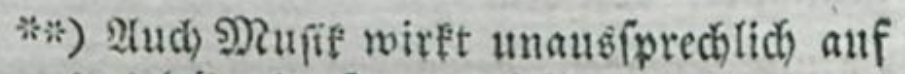
Das leibende $\overline{\mathcal{F}}_{2} \mathrm{er}$. 2 Ill mein frumpfes Szimbriten, die eiferne eimfonneidenofte Epanmung, aus ber midi) oft 9itdots beym Tose meiner geliebten Sd)weffer berausbringen founte, verlolye fich ous

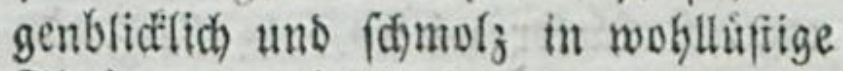
Thlánen uno in fanftes Scinmeigen zuF Siube oder zu fitller f̧eiterkeit, wenn פius fift in weidfen 9Retosieen midh einwiegte ; unausfpredglich wohl waro mir immer fobalo mide Rlopfiod s 2luferftes hungegefang wefte ober fobalo mein Freunb in füsen umb ertyabenen જुgan= tafieen meine Seele jum Srimmel hob; nod) Danft Dir mein $\mathfrak{f e r}^{2}$ für Diefe Troffungen, guter, theilnehmender $\mathfrak{m * *}$ 


\section{8}

ten itsn weifer uns gluctlich. Reipjig I791. - femue id feing, das mebr ben Geprestan empfoblen merben fann, als 3 wect.

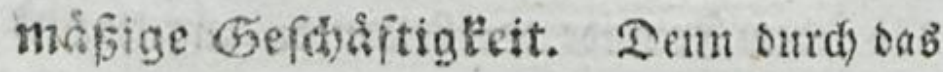
Bewupterenn: auch beute baft Du dies und bas (Sute rieber vollbrad)t, (uno bie traus rigfre Eccle findet gewiß in fich defe Erinner ruแg) nud beute wieder etwas jur grofen Bhitfeligfeitsfumme tor 233 eit bengetragen,

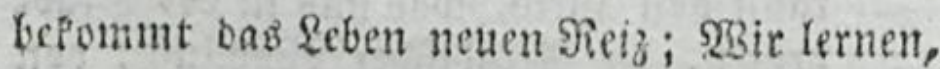
baf es aud) a uffer unferm verfolyrien (bies genfrande, voul dem wir wiknten, onj mur

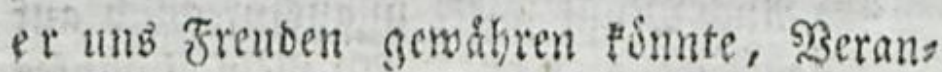
foffung zur Frende giebt, unb oá̧ es nur von II $\mathrm{s}$ abbånge, fie แแเ zนzurignen. Demn bies fer Gedanfe mus auf gut geartete Seefen ungleich) ftátfer wirfen, als alle Berfreutu, gen, ia felbft alle SBergnuigungen ber 2Belt,

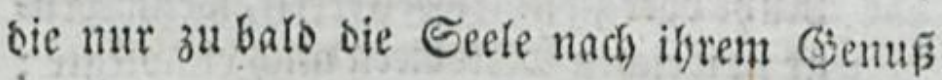
in ibre vorige \&eerkeit zurid fallen raffen.

Thatigtett alfo ift bas grose gol= beue SRittel, bas bey jebem Traurigen nicht blö gyalliativcur bleibt, fondern bas Hebel ans sem Grunde heift; in, es hat nod ben

23ors 


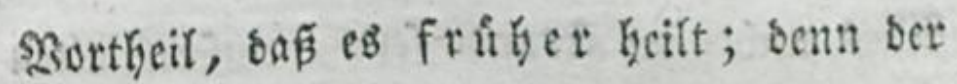
wabre Seelenzufand ber meifen Rraurigen if ber: fie fublen fich allein, nir= genos - Fein altachement, nirgenos glaubent fie Erfas für bas şerlogrme zu finden. (5) $e=$ $f\left(c^{2}\right.$ afte bingegen - unb ie woblthatiger für Dab 280 by 2mberer, befto beffer fur fie zu bicfen aruect - feffern wieder an Leben. Biel fdjon gewonnen, wo Das gerwonnen

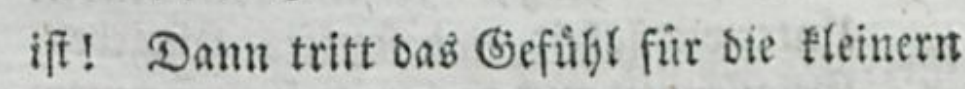

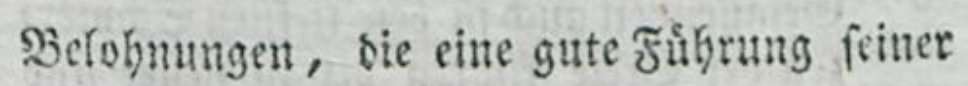

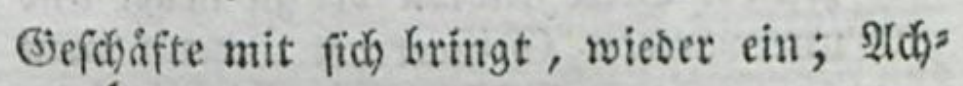
tung 2 tnbrer, 2usicht fur bie guten fole gen berfelben, \&iebe fold)er જुerfonen, benen wir vielfeidst Dienfte reiften funnten -

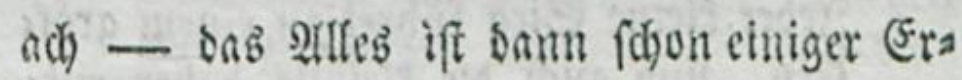
fars! ङB fint ja ouch nod einige frentent Derfelben fir uns aufbebalten! - Uns wab fur Freuden? - Selbferworbene! :

S wie bas unjerm Stolz fomeidyelt! -

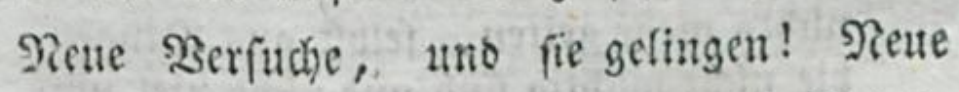
2ufrengung unfrer frrafte, uno bie Belofya nung bleibt nicht aua! SBie folfte bกb भlle? 


\section{co}

शllles nidjt in Stanbe feyn, unfern alten Sdbinerz fruber zu beilen! *)

Freunde

*) Einfameeit vermindert gewis iurés gelinde 2 ufrengung uno fanfteg (B)egenfrteben die Szelancbolie, fo bald man ficts sum (befers macht, in Der: felbeu nicmals minitg 3 u feyn, mit Geould fein Reiden zal tragen, uno im Etillen immer et $w a \mathfrak{b}$, fo gering $\mathfrak{e b}$ alic) fenn mag, zu unternebmen. Uluterwitfigfeit unb fanftes Singeben, Belaffenbeit auch in ben tieffren Edomer: zen ber Seele fulbren oft zu neuer uno unerwarteter Beiftesfraft, wenn man fich nur: oem (Eçel gegen alle 23 irffams feit nicht úberlást, uno nie aufbort, ourch einen gelaffenen aber forfwabren= den 2 Biderftano fein llebel zu befámpfen. Seber fleine Sieg fúbret zu einem grós: fern, uno aud bie fleinfte baber ents frebende Freube unterbridt fobun wenig= frent ons (jefúbl einer immerwaburenten Untuft. San mache fich, wenn aud alle Erforderniffe ber $\mathfrak{B}$ ermunft uno ber sugeno gegen Siranflbeit uno ibble Raune nichts mebr gelten, felbfi aแв Dingen, oie nicht widhtig fino uno nicht viel um. faffen, ein (Biefchåft, benn taufenbfas der Llnmuth wiro oft plög̨lich burch cine febr geringe 2 Infrengung verbrangt.

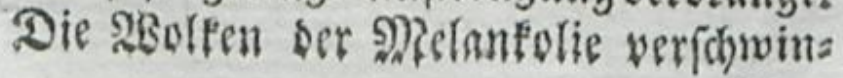

Qen, 


\section{1}

Freunbe ber Reibenten! EF ift nicht

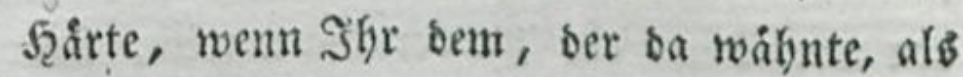
babe ber Too ifm 2flles gernubt, gleidjam

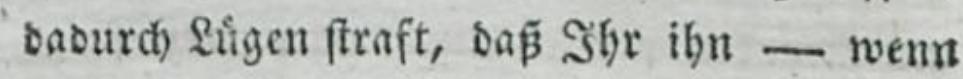
altid) mit einiger Gewalt - binfübrt, wo anbre Freuben feiner warten. Freiben, fúr

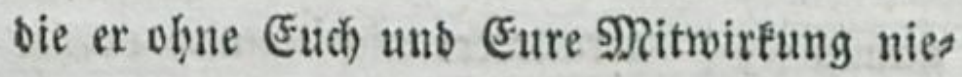
malz Sinn wuro befommen baben, weil er

fie

ben, weun man nur einiges Sntereffe an irgend Ctwab finbet, Dab man mit Dem grósten 2 Biberwillen unternimmt.

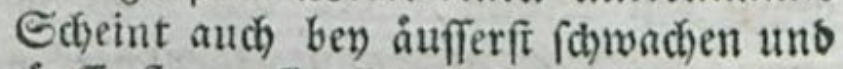

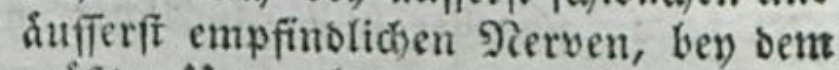
grósten Ulmmuth uno bem allervollém= menfen Heberoruffe alles \$ermógenzur Riegfamteit ganz vernichtet; erliegen and) bie গierven unter ber Eleinften 2 (n) ftrengung; fo ift ood) gervis felye oft,

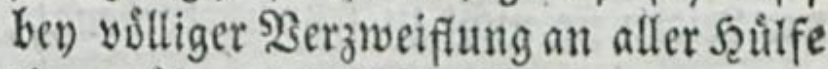
bie unibernindlidffe Albneigung gegen allen গath uno allen Troft, biefe ltns regfamteit des fórpers, Diefe \&åmung ber Seele, niđ̆tż als eine Bemántelung unfrer úblen $\mathfrak{a} a u n e$, uno alfo etne wabre Siranfbeit ber Imagination, bie mant blós ourch einen feften uno geftáblten WSillen gegen alle Erwartung áberwinbet.

Zimmermann- Heber bie (E) 


\section{2}

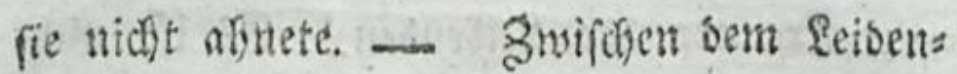
Den unb Den, bet ifu berubigen will, finz

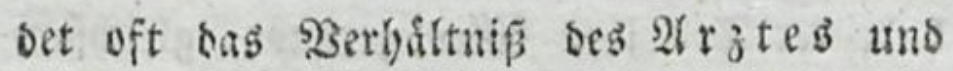
Des Siranfen ftatt. Ein fluger 2trit fragt niçt erfi: ob biefe ober jute Trzney ber oft eigenjinnigen Conyenienz bez Firanten gemaíz fev; zergliebert nicht immer vorker sen erfien Einbrud ber 2 rijney alt bie frans fen leibenden skeile, funbern ex ha no elt. Der Siranfe f(i)mábet yielleidyt im 21nfang; aber bals legt fich fein linwillen uns Dant gegen ben SRann, ber ibn nid)t f(bonte, nber befro faneller uno gludflicher beifte, tritt mun an die Stelle beffelfen.

\section{3.}

Itnglidflidse uns Reibenbe firts oft, went Dab \&eiden fie nicht zu febr niederbengt, febr feid) $\mathrm{tglaubig;-fie} \mathrm{feben} \mathrm{in} \mathrm{jeber} \mathrm{Nosigs}$

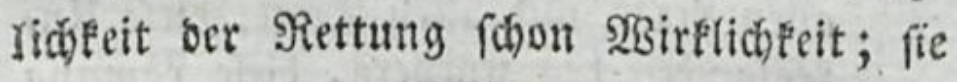
bordben auf ieben Sinth, ergreifen gern jebes Mittel, wrib man ifnen zur Erleichterung

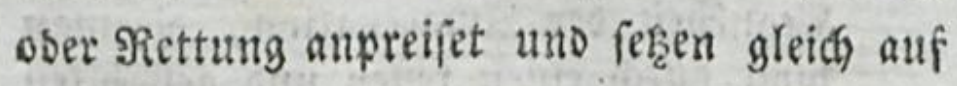
jebes Nittel ibre ganze f̧offnung - Saß fie in Diefer Stimmung nie aub den \&ugen, wers

wabr 
wahte fie sor vibereilten Itnternebnungen,

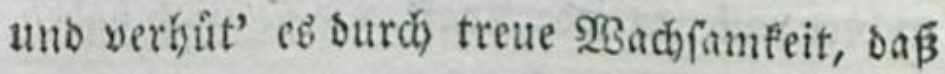

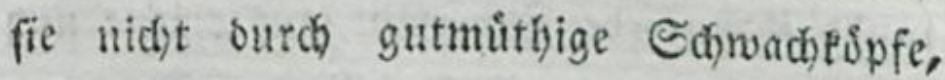
woer burd) eitle, Teichtfinnige, unbefonnene

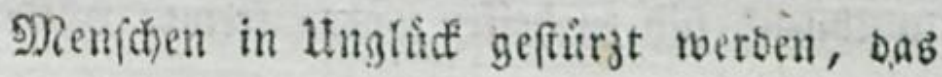
árger ift, benn onb erfie.

Sft seibenden biefer Aft aber einigemal

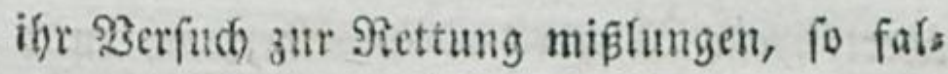
len fie Yeicht in Das anbre Extrem, fie glaus

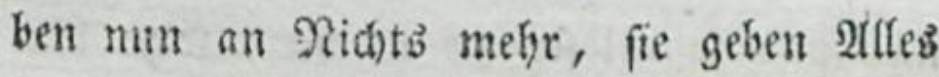
vertogren; *) es fehlt ifnen, fo wie meift allen Diefgebeugten, gemeiniglidy an aller Suverficht bey ihren Unternehmungen, fie find foreu uno zagbaft und mutblob, fehen

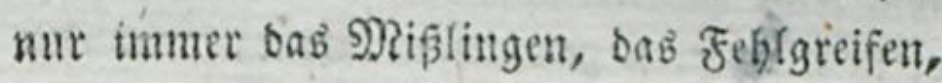
fehen uno fuften jeoe Schwulrigfeit boppert uno unúberwindlidh, uns fudjen uno finben diberall şinberniffe, two feine find. SIll ift Siraftgefuigl liegt todt in ihnen, uno oas peins

lidie

*) Quid nimis miferi volunt

Hoc facile credunt.

MEG. Immo quod metuunt nimis, Numquam amoveri poffe nec tolli putant.

Seneca Herc, fur, 


\section{4}

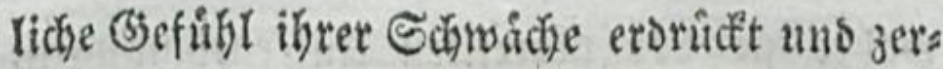
nidbtet yollenos jede Siraft, die fie nod) baben, raubt ifnen jebe Suvericht auf eigene uno

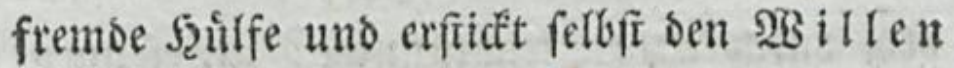
in ignen, fich zu belfen, ober frembe Şulfe anjunetgmen.

Szite Dids einen folchen Seibenben burch jibertriebene $\mathscr{B}$ Beidbeit Deineb Serjens, Durch

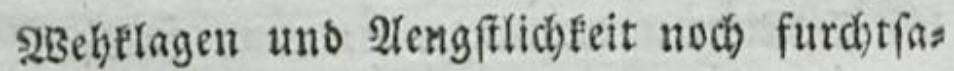
mer uno mutblofer zu machen; zeige ibm Entfoldoffenbeit, Nutb uns Feftigfeit; reißs ihn mit Bsewalt aus feinem গichts beraub; wecfe in ibm Buverficht zu fich felbft; zeig ifm feine Rraft, die nod in ifm liegt, went er fie nidbt felbft gewaltiam láhnt; fprid) ikm Sicht in bie Seele uns Muth ins $\mathfrak{F}_{\text {erz }}$; leite

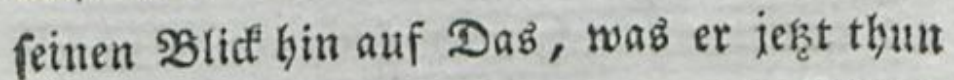
tann, thun muईs, wenn or noch Mitleis sen verbie: en und feine unwuirdige Nemme feyn will; f(c)meichele feiner Sd)wåd)e nicht;

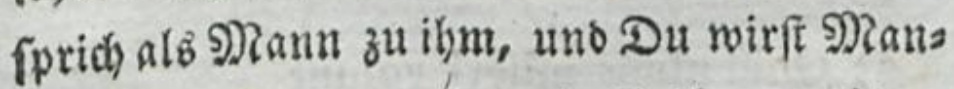
nesfinn in ibm weden; (uch) ifn zu libers zeunen, oá̃, menn gleid) feine Sdimerzems prinoungen wabr fino, Dap Dod siefe Em= pfin: 


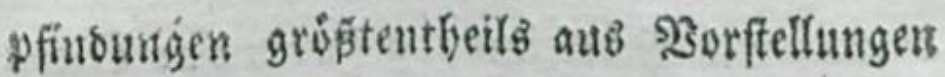
entfpringen, Die falící fint.

f̧ite Dich) aber babey vor llebereiluns gen uno Blófen, vor Trugfdliffen und Sos phiftereyen, woyon er Dir bas biegentheil Darthun fann - bủte Did), Erwartungen uno feffnungen woll 3 uver fich $t$ in ihm ou beteben, ibre Erfilllung mit (3) ewi zu verfprecben, wenn fie noch felyr ungerwif fins ano feblidlagen fonnen. - Eine falíd) Borfellung, auf oer er Didi extappt, ober Eine fehlgefhlagene Şoffmung, beren gerwiffe Erfúllung Du ihm verbief́eft, fann Dir mit Einemmal alles Butrauten uno alles Berwidgt über igu rauben.

I4.

Beyfpiele mirten auf ben Leibenten oft unbeforreiblidy - fie, redit gebraudst, fónnen plosęlich alle feine Empfindungent umftimmen, uno felbft ben Nuthlofeften mit

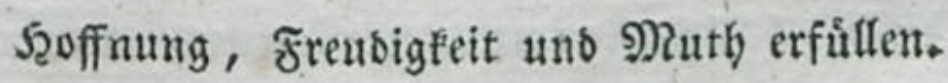
2lber zu शichtb ift ber Leibenbe megr geneigt, unb nie iff er fdarffictiger unb fos phiffifder, als da, wo es auf bie Jzerabwitrs 


\section{6}

sigung und serfleinerung fre tu ber Leibeit

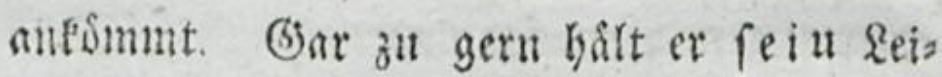
sen fur cimzig; foldgen Serfuft wie er lei= set, bat noch nie (siner erlitten, fold)e Ed)mergen nod) nie (5iner gefiblt, fein Ltu= gluct if mit feinem zu vergleichen, unb barum fann or fids sen sroft, ben andere Anglice liche fanben, nidht jueignen, er paft nicht auf ibn, bie Shulfe, bie Sene fanden, wos burch Sene geretfet wurden, fam er uiddst boffen, sie llmftanse waren bort weit glude Yicher, bie Laft, werche geljoben warbe, weit

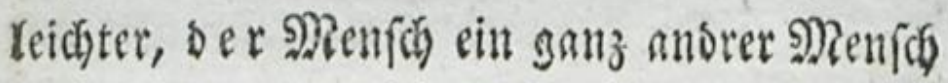
alb er 25.

20ăble saber nut folche zeyípiele 3 Deinem Jroect, bey senen er (d) lect)= teroings, wo cr nicht ein grōjers,

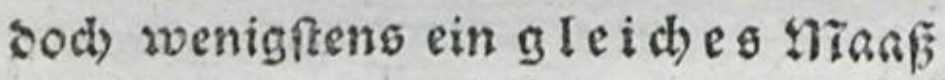
von Lciben, vou Gefalsren, von orit: Fenden Derlegenbeiten, von Ziatb: uns buiflofigfeit anerfennen muf, wo 23erubigung, Troft, Sculfe unb Rettung cben fo ungewifi, eben fo umwabrfaceinlich zu fenn fajien, als bey ifm, uno wie fie an Ende ood erforgte.

Fืa 
M3áble diç Bevipiefe wo móglid aus

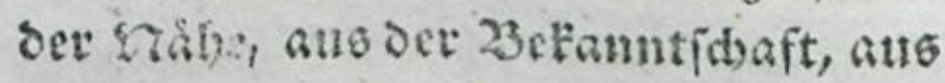
dem Gefidtstreife oes Leibenoen; fie

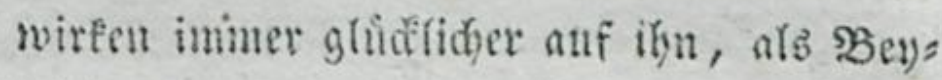
fpiefe ath ber Fome; or foum fie felbft beffer prifen, mit feinem Suftans vergleidyen uns nuff fici) amwendert.

28seift Du in Deinem Steife einen ed=

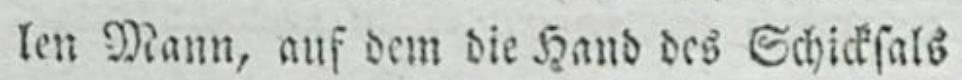

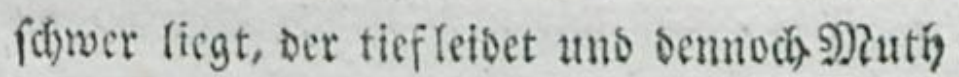
bebaft uno feft an (5sott glaubt; fo eile mit Deinem leibenden Frembe 3 ifm, unb er wirb yon ifm fermen. — Dab $\mathfrak{B e y f p i e f}$ eineb

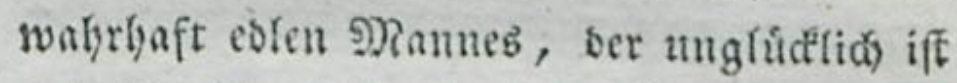
unb fein Lungliet máunliá) trígt, wirft auf 'ein zartfithlenteb leidendeb frerz $_{3}$ oft unendlich

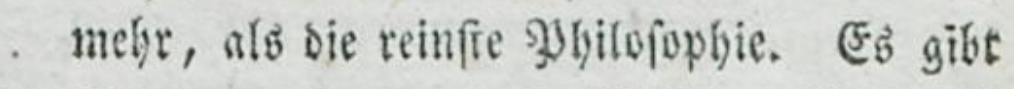
Stumben, in benen bie Religion felbft alle Sraft an แนถ vertohren zu baben foceint; in Denen die beßsten wirfjamften Troftgrinbe an Der Dberfidde abgleiter. 2tber ber rubige

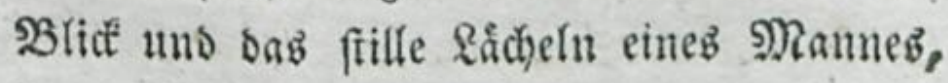
sẹn, cben fo, wie unbె, bie fohmerzlidffen feiben nieberbengen, sie fanfte Szeiterfeit, bie (ए) 2 ex 


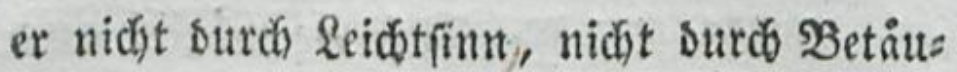
bung, nicht ourd) abgehårtete Fúbllofigfeit bie er ourd) oen ebelften ḩeroisomus auf féne Etime locfte - o gewis ! fie erwarmt aud) uns, felbft in ben finfterfen Sugenblicten, mit Muth, mit אiraft, mit Freudigeteit, Szoffs nung und Buveriidt; alle Febern, bie ge, waltfin niesergeoruidt maren, foringen wiez ber alf; wir füblen es in bem 2fugenblide

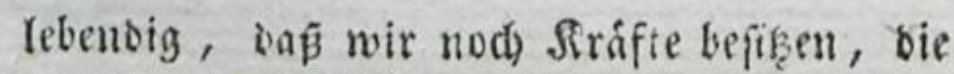
wir bishler nidgt benurzten. Der Bebanfe:

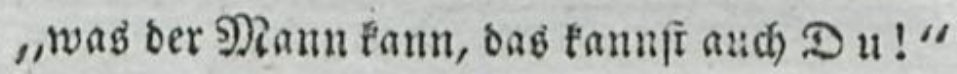
gibt uns eine (Entfóslofientyeit, einen eifernen Trof̧, ber uns mit neuem uniberwindidyem Neutbe belebt, baß wir Allem fect die Stime bieten, wab uns niedertreten will. -

Feblen Dir foldye Beyfpiele aber in bex Näbe, fo finieft Du fie in ber Befdrichte, bes fonbers in ber biblifchen befdidte ges

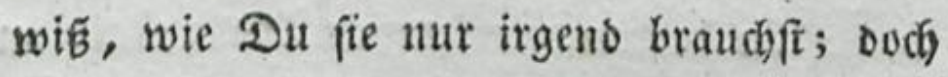
wirfen Beyfpiele aub ber $\$$ ibel meift inmer weniger, aโs Beyfpiele aus bem gemeinert Reben; ber Reibenoe lasalt entweder jene \$ens

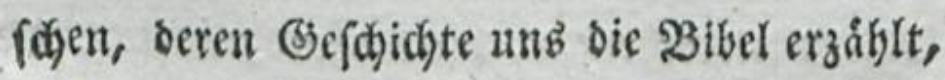




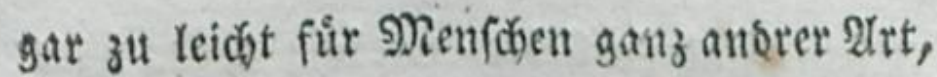
ober er glaubt, (5ott habe bamalo bie Miens fhen weit mefre im 2luge gebabt, unnittels barer fid ifhrer angenommen uno fît fie ges forgt $2 c .2 c$.

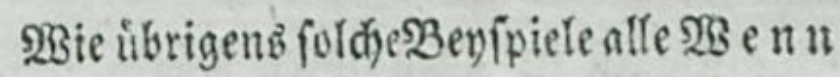
unt 21 b e r beb \&eidenden nieberfalagen, wie fie ihn plob̨lid aแb fid feltht beraubleben, fein $\mathfrak{S}_{2}$ erz erweitern uno feinen Bieift beleben fómen, - bavon madite id oft in meinem \&eben bie fifsefte Erfahrung.

Bgillft Du aber burd Beyfpiete troffen, sie ber Rage Deineg Reidenten niđat vóllig gleiden, fo wirfi Da ficher von ifgm fo surúd getricben, wie 2 ntonto won Leo= nato beym Ebafefpear: *) ,I็

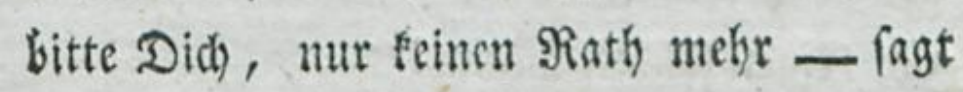
ber troftufe 2 in to nio - er fallt frudits lus in mein Shr, wie 28 affer in ein Sieb.

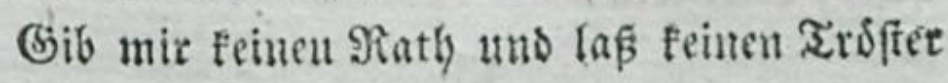
meinem Sby fasmeicheln wollen, als nu $\mathrm{r}$ einen Eolden, deffen unglutet sem meinigen

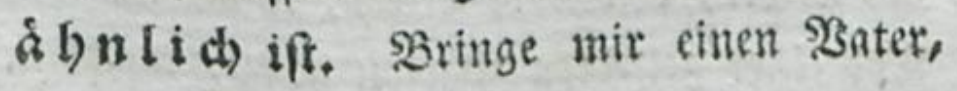

$$
\text { E } 3 \text { Det }
$$

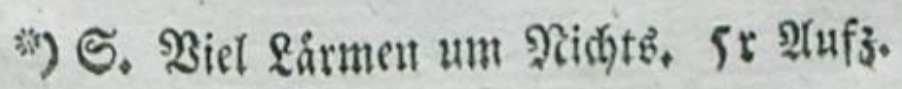


70

Der fein Sins fo Yiebte, wie ich; seffen freu be an bemfelben auf eine foldse afrt zers nid)tet iff, uns beif̧ ifn bann von (joburs teben; lafi ifn feinen Eckmerz nach ber Lange uno Breite bes meinigen abmeffen, uno laf feine filagen in allen ibren Tonen mit ber neinigen zufammen ftimmen, als Dab fur Das, uns ein foldes SBBeh fur cin fordbes in jedem 3uge, jeber Giefralt uno form: went fold) ein Mam lad)elt, unt feitten Bart ftreidfelt, uno ruft: fort mit ben Summer! Ino han! fagt, wesm ex adbzen folte; weut er feinen Gram mit Sprucbwotern tiberaflas

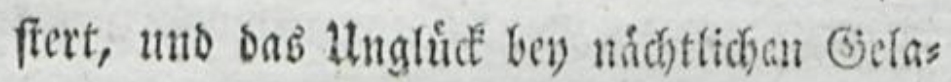
gen bermifat - fo bring' ifn zu mir, uns

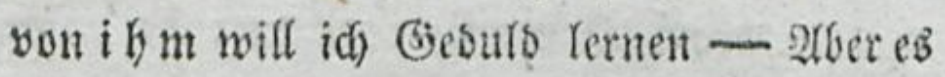
gibt feinen fwlben s) Rann!"

Dab if immer bic gुJgilofoplyie cines Keftig \&eibenten! -

\section{I5.}

నiefes ourdy Mrate uns Seele greifen= จez Leiben, befonocrb Reiben, in sie unb ber Xus ciner geliebten \$erfon frurbt, macht in oet 93 uth Des Sdsmerzes nidjt nur gleids= gîl= 
gittig gegen alles sunere Glüt, fon Dern ç flóst oft jogar gánjlidbe abneigung Dagegen ein. Der Reibente mag nicht bू ba won boren unb fegen, bafe won auffen her nod

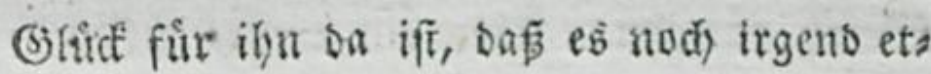
wab gibt, was feinem Seelenleiben mur eis nigermapen baß BSteidggewidht geben fann.

Man hute fich alfo, bey foldyer Stim= mung âtuffere Glưctoumftanoe, in benen Der Seibenbe fic befinbet, ober bie er nod) jut erwarten bat, zu Feiffömitteln ber Serufi= gung z̆ wâblen. - Sie emporen ifn, fatt

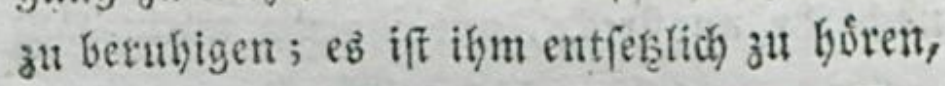

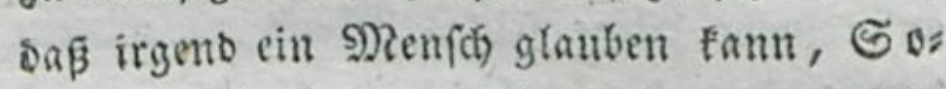
etwas fonne ibm Erfakg geben; er fúbit (iic) unbefợreibliç) beleibigt uno gefrånft, uns Der Menfo, ber ifu fo zu tróften glaubte, wito ifm unteiblich uno verhant - Eeiti

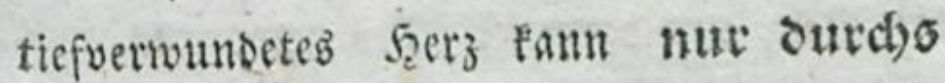

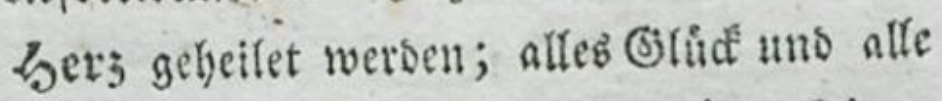

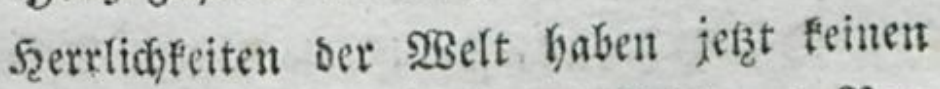

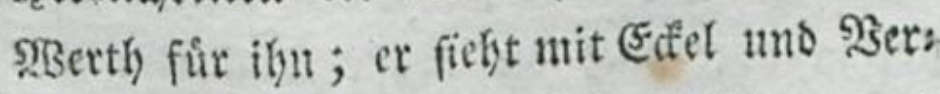
achtung auf fie bin - Sein \&idbfes, wab. er Gatte, if vertobren - liegt ill Braber acdi) 


\section{2}

ach - mit ifm liegt auth alles sfluct bes Rebent im (Srabe - Fein Reid)tbum, Fein

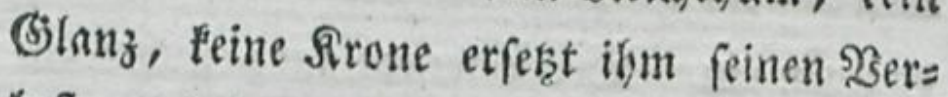

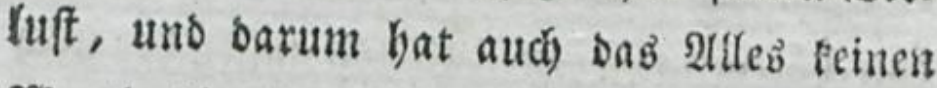
23erth) fưr ihn. 2lber ein trette theilnely=

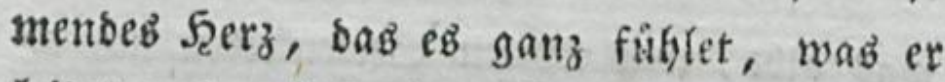
leibet uno voll innigen Nitreiob fich an Dab

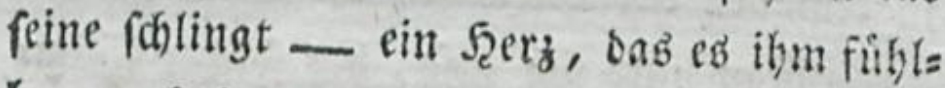
bar macht in tieffien Ceelenf(d)merz - ể fẹ ijm oudh nodh nidgt alle fiebe abgefturben, er babe bod nod) eine treue Eecle um fich, bie ihm entgegen eilt, wenn er feine Itrme nach) ibr ausftredt, uno ifhn nie allein last allf feinem $23 e g e$ feines $\mathfrak{L}$ bens -0 , ein folches Şerz bat nod 2 SBerth fưr ihn, es if bas Rofftid)fe, das Einzige, was ihm librig Glieb - Uno surd) Diefeb Fettet er fird alls málid) yon nettem wieber ans \&eben und an

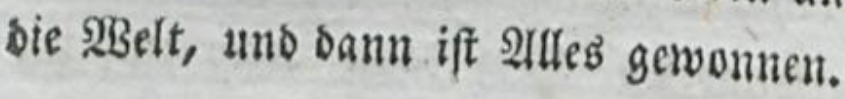

\section{I6.}

Die ஒु fretimn aber aucb bie árgfte Duaflerinn bes Reidenden, befonberb be в Reibenben, oer ein

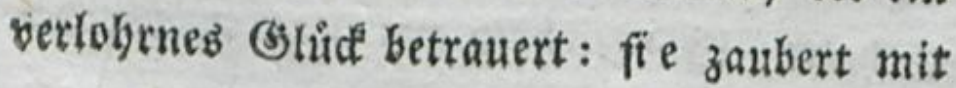




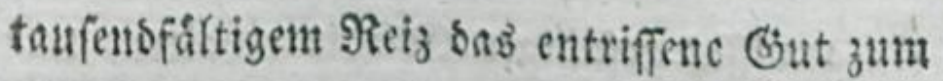

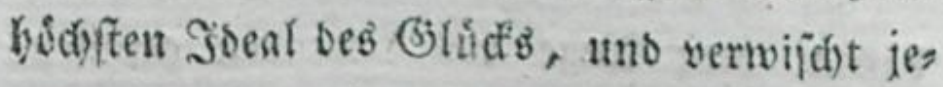
Den Flecten uno jesen fobler an ihm, bis auf sie fleinfte Spur; fie bringt ihm oab হ̧er= lobrne immer nab uns gegenmártig - er fielst uno birt uno fuiblt uns benft nichts als feinen Dseriuf und oas mit ibu verlobrne Gluct, uno alle bie Tage ber Jreuben, die

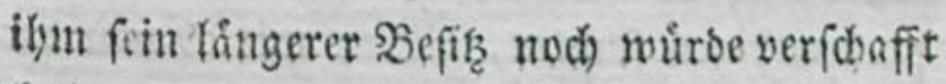
Gaben, uns das Elens, was jeşt nun feiner wartet. - SBer alfo cinen foldhen Reibenben,

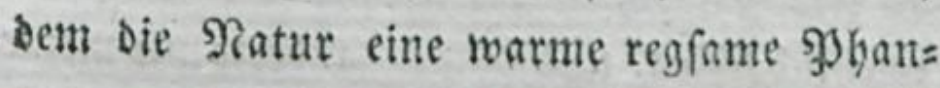
tnfie gegeben bat, wirfan troften will, oex mus otefe Pbantafte aud) 3 feinev freunbinn wåblen, um durb ibre builfe das fotswere wert defto fisnel lev 3 u befteben.

Scite 3idh baber, jenes Soeal verfleis nern uno, ifm ben হerluft feines (B)lüct's ges ringer beredinen ou wollen. Suche vielmebr

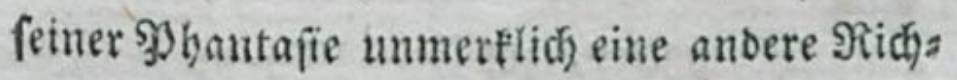
tung zu geben; feşe zu ifyren Giemálben, woburd) fie ben \&eibenden anjiebt uno tåufdt,

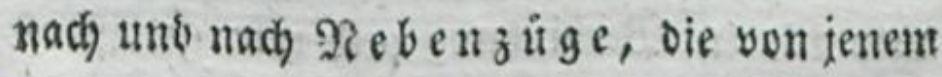

$$
\text { ङ } 5 \text { ein }
$$




\section{4}

ein gewiffes gefálliges Eolorit erbalteit, uns barum bem 2luge bes Reibenden gefallen;

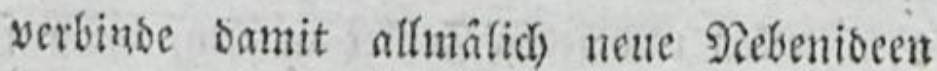
uno leite ifgn fo unmerflicí) yon feiner fraupts ibee ab.

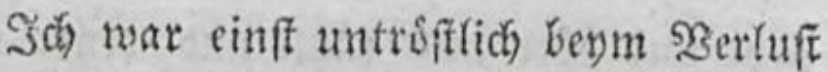
einer gelicbten Edjwefter, mo alle Trofts worte, womit meine reblichen Freunbe mich 3"l berubigen fudbten, glitten mix ab von ber Seele uno feins wollte haftert. Simmer fúfllte ió) mur meinen \$erfuft, ber mir fo gan erfęlich fobien, uno inmer zauberte mir meine

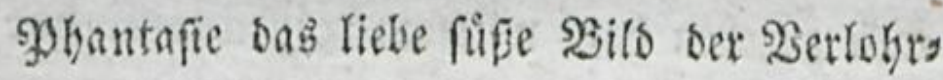
tren wor 2fitgen, uno Seber mat mir tmertrigs (ida), uno Seben flob' id, Der mir's surch

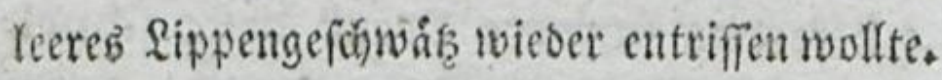
2 tber ats endich eine theilnelymente Geele

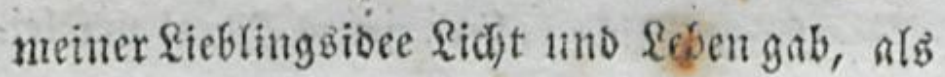
fie mit ben (Bebanfen, ber mir inmer in ber

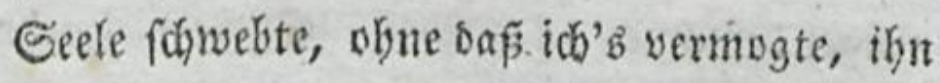
ganz feffzubatten, zur theberzengung brachte: meine vertlarte Sdimeffer fen felbfe surch ben Bub nicht von mir getremut, fie fer) iefst noch mit mir in Nerbinsung und mir gewif oft

un= 


\section{5}

utifidt tbar gegcuwartig - sa waro id) uns merefict) und leife von bem nagenden (S) furly meines 23 erfuft's abgezogen - je fefter unb inniger fid Meitt Szerz in biefe Sorftelfing bincinfog, sefto wentiger glaubte id verlofren zul braben - ,fie lebt ia nod), lebt ia noch „fur Didf uno Du fur fie - eb ift ja nicht "Siremmun, wab Du Yeiseft, fie ift in nod "bey Dir, fieht Didh, Gort Didy und bort "nid) nuf Did zul lieben." Etunben lang fount id mit ifgr fprect)en, mit ifs mich une terbalten bis zur gâizlidgen Sergeffenbeit

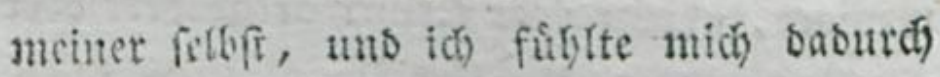
Fimulifes geboben liber nlleb srauren uns fiba aflen Sdymerz. - פJogte oab immers bin Edybarmerey feyn - fur midh war es feine fobliche - o es war mir cine unaubs fpredslidy wohltbatige ๔d)warmerey; fie gab nuir গiube uno seben wieber, uno machte mich allmálich wieber fiffig, nnoern lleberlegungen Ramu ju geben, unb ferb ft baurenden Troft oa zu fuchen uno zu finben, wo ihn ber gute Gott feinen Sinbern anteut, - 
I 7 .

Şat ber Leibenbe nod grofíere seiben zu furchten, und feblt $e \mathfrak{b}$ ifgm nicht gants und gar an Sraft, audd D a s nod zu ertragen -

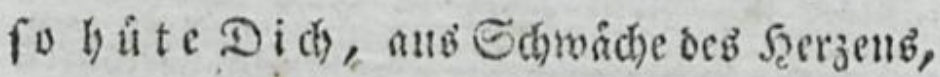

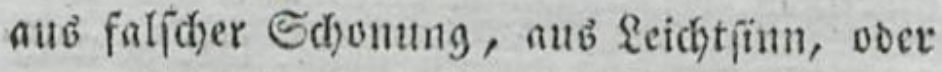
wab fuir Uhradyen es feyn mógen, die Ge= foblen ibm geringer vorzuftellen, alo fie wirtich find. - Iluf Den, ber ettr? mal furdstet und in biefer Furdith fdon leibet, madien felten folde sorfiellungen fobmerzlis d)ern Einbruct - eine befalyr mehr ober weniger, grofer ooer fleiner vergro̊pert ober vermintbert fein llnglied im Baanzen nidgt,

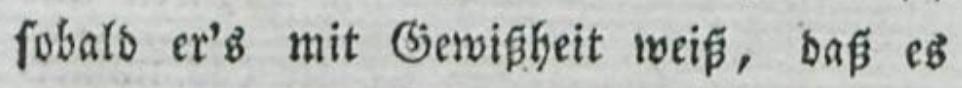
f(c)lect)terbings unvermeidlid) ifr. Und oft ift és ein fefhr grop̧er Troft im \&eiben, bas \&eis ben auf Einmat und $g a n_{j}$ überfehen, uns

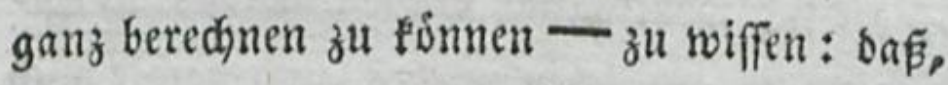
wenn $D$ a $s$ nun einmal úberfianben ift, body bann weiter feins 'melyr forgt - *) Man macht fich ba auf 2 tlleb melyr gefaft, man

fielyt

*) Saepe calamitatis Solatium eft noffe fortem fuam,

Curt, I+IV. 
- fiefte attes melyr yorker, ninimt feize Mråfte beffer zufammen, um den Sajlag, den man

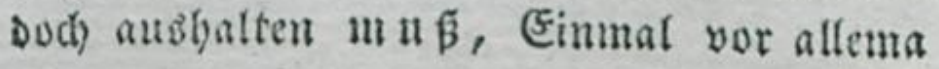

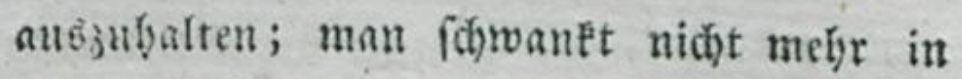
ewig peinlicher Unrube zwifhen Furcht uno Szoffmung, wab alle Fefigigeit raubt, alle siraft zeroricăt uns um alle Entid)loffenbeit uth bringt; uns weil wir nidgt mebr boffen, fo (d)ligt unb auch) feine froffnung mehr felgl,

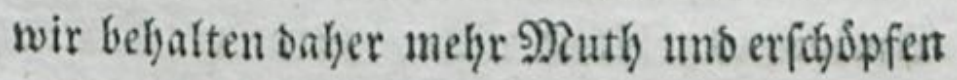
unfre Srafte nicht in vergebliçen Berfus

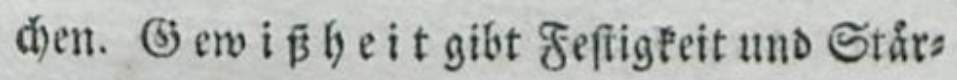
te, uno man feibet immer entfhloffener, wils liger, getroftern Matty, wem man's gewi weif, wie viel man ju leiben bat und wofin eร am Ende fúbrt. -

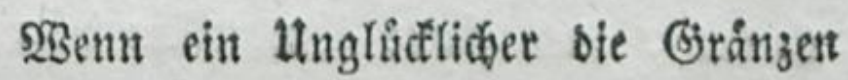

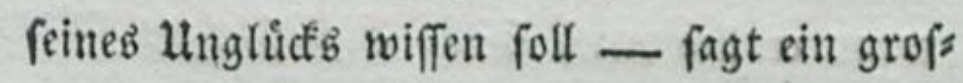
fer Sentuer ber \&eibenden in ben Lebens= låuffen nad) aufiteigenoer Linie fo meft fie ibm ganz uno gar ju, feinent Strid) weniger; SGr madet ifun fonft ben jes Dem neuen auge unglúflidjer; Şgr lap̧t ihn einen 


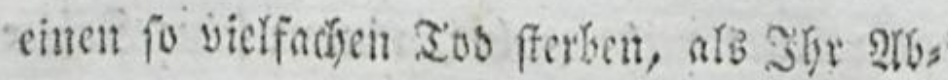

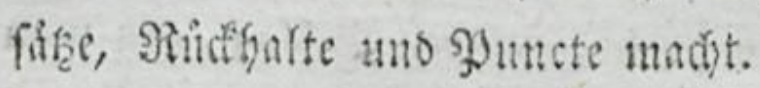

I8.

3asucife sie gemouefe 2tupmert. fompeit, wenn Dir ein Lcibender die Gejetidte feiner Leioen erjoblt; ibers hyore unb vergif feinen wid)tigen thmftanto Derfectben; unterbrich ifn nicht mit Suects fragen unb franfe ifn nicht mit verfechrten 2tntworten; likd) nicht, wenn ex Dir oft Slleinigfeiten ats Dinge you ber grơftelt

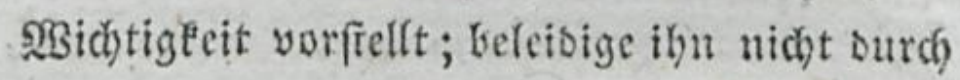

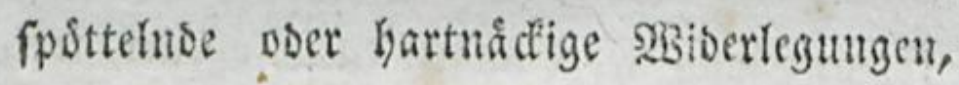

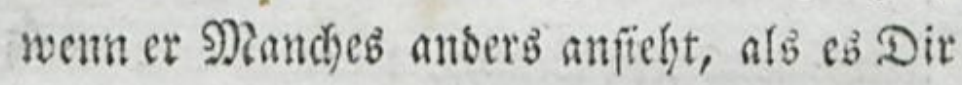

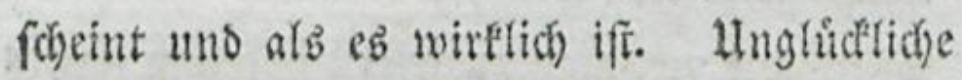
werben leid)t gefownergis, wem fie eimmal ins Resen bineinfommen, - ertrag sas mit Bebulb, unb gône ilym willig bein SObr, wem er fid ourdh folche Ergiejungen feiner Empfinbungen erfeichtert fühlt; werbe niḑt

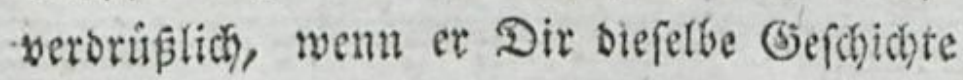

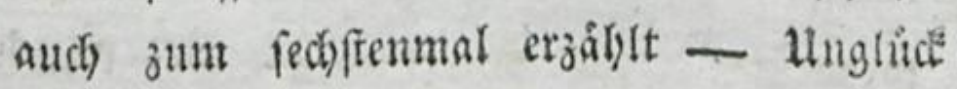

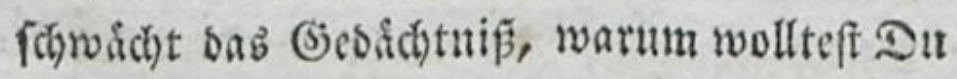

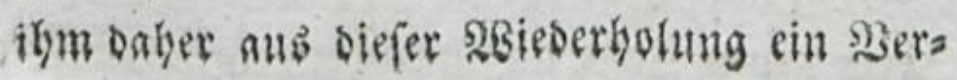

bre: 
brecken madben, oa fie ein $\mathfrak{B} e$ weig mebr von Der Grife feines feibens, fo wie von bem 3utrauten ift, bas er gegen Did) bat?

Szandelteft Du wiber Einen biefer જुune; te', fo wirdeft Du ifm shangel an Theils nabme verrathen - e'r wütse es wenigfiens bafuir balten, auf Did) erbittert werben uns Dir fein ganzes Butrauen entzieken - uns Du vermagf bunn शichts mebr uiber ifu.

$$
\text { I9. }
$$

So verfकloffen aud oft bas Itnglice on Ieibenben madst, fo tief wie es ibu oft in fich felbf bineinziebt, fo offenberzig uns mit. theilens wirb er aud oft auf oer andern Seite, went die Dámme feineb Schweigens eimmal gebrodhen fins, und fein Innres fich ergieft in vertraulicbe Rlagen liber Seelen: ficherz uno gebeime Noth. Itnaufbaltfant fromt onum aแt ify heraub gllteb, was ex fo lange in ber Tiefe feines $\mathcal{H}_{2}$ erzenb gerwalts

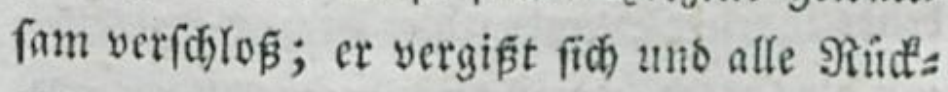
fidften uno alle şerbaltniffe, die biaber feine Bunge feffelten, unb entbectit mun felbft bie widbtigfen Bekeimniffe, yon benen oft feine

ङgre, 


\section{0}

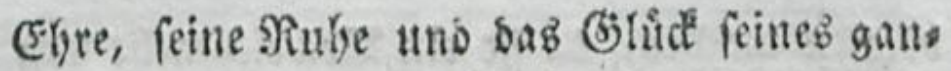
zen Rebens abbangt, uns bie er unter allen

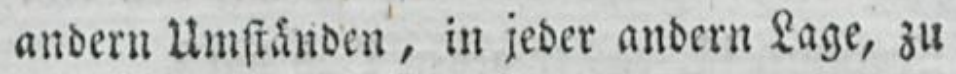
jeber anbern Beit nimmermebr entbect ba= ben wårbe.

Zetracte folde Geftandnifie alo oas unverleslidyfte beiligtbum, was sir conbertraut wurbe. (5: iff fdo ánblich Int niebertrâchtig, an irgend cintem Menfchen

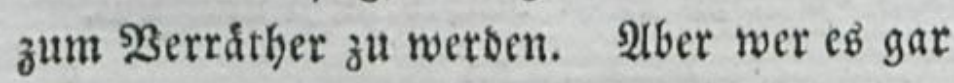
all einem Unglúdlidben unb Seibenden wirb ber $\mathfrak{3}$ ube macht fich ewig unwerth afler $2(d)=$ tung uno alles Sutrauens uno aller Sूerbins bung mit Menfchen - er wirb an ber Menfch= beit felbft zum \$errátber, uno unausldfoliche Sd)ande brandmarfe ifn zur 2 sarmung fur Seben oer ilym nabe fommt. -

Rein $280 r t$ und feine Sylbe vou ifun entfdlupfe baker ie deinen Rippen. Selbit bie gleicbguItigen Dinge, bie etwa ber $\mathfrak{e}$ is benbe in jener Stunbe feiner $\mathcal{F}_{2}$ erzenzergiefung mit bir (prad), Darfft ou feinem Anbern mits theilen. Der Leibende ift angfilich unb arg=

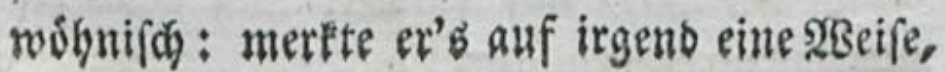




\section{1}

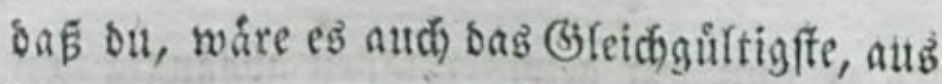
iener Stunbe verrathen hatteff, fo whirbe er furchten, baßs bu aud wot nod Megr uns

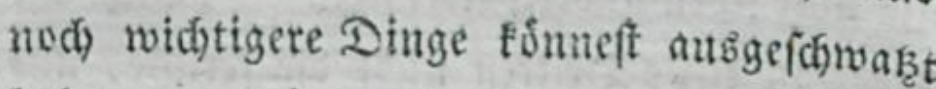
baben; er wúrbe bir fein ganzes autrauten entzieben uno fid unbef(dreiblid ángfrigen.

Sft bereutet eś ber feibende fdion in ber

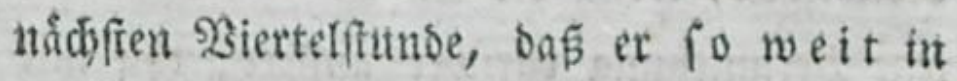
feiner Sistraulidfeeit gegen otd) gegangen ift, uno bir Dinge entoectit bat, bie fein Silité und feine Eyre ganz in beine Gerwalt gabert. -

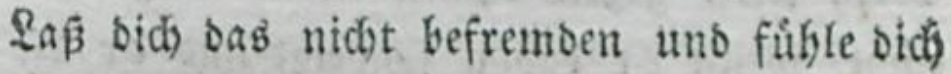
baburch nicht beteidigt. (5. if nicht grabe

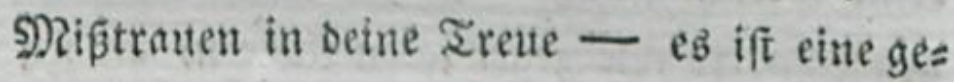

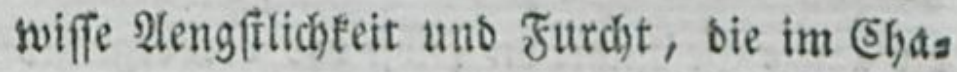
racter faft aller leibenben liegt. Thut bu mut

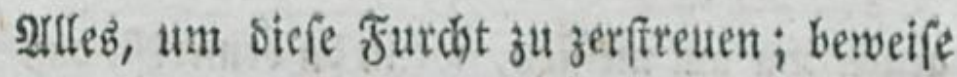

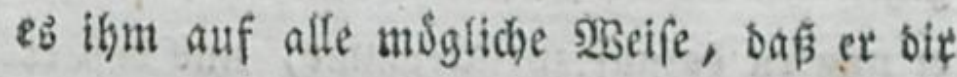
ganz umum/dyrantet trauen fann uno belgandele ibn in allen Dingen mit ber feinfen Delicas

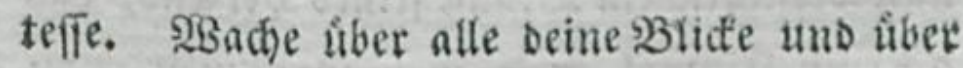
alle beine Sinen, uno vermeide 2(lleb, wab bir ben Schein geben fónne, als wolleft but

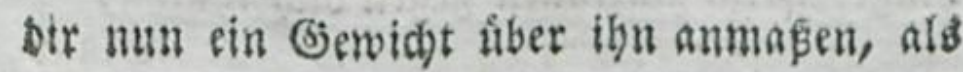


82

fey er nutn jegt gewiffermapact abjangig yout

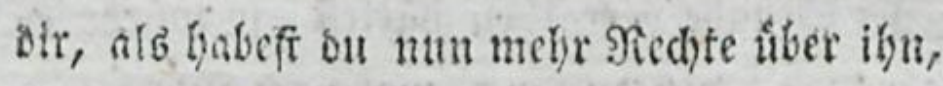
atล Lúter feben anbern fremb. - Macbe im Deyfenn cines Dritten nidyt bie fernfre, una merflid)fe Sthpielung auf feine oir anvertraute (befbidste; wolle fie weber beftatigen nod berichtigen, wenn 'bir cin 9 anberer biefe boer jene Bermuthung Dariber áffert; thu mit aller $\mathcal{H}$ nbefangentheit, bie bir móglida) ift, ais swiffert bu gar Midits savon; beribre fie ofne Noth felbft gegen deinen letienden Freuns nidit, wenn er bavon fofweigt und er bir feine Seranlaffung bazu gibt; thu aud ges gen Shu, fo wie gegen jeben 2 (ndoern, alb fir) bie ganze (seföbichte bir felbft bas verburgenffe (B) heimnis, unb beweife foldhe Feinlgeit Dann gegen ifg am meifen, menn ou bemerfft, onf

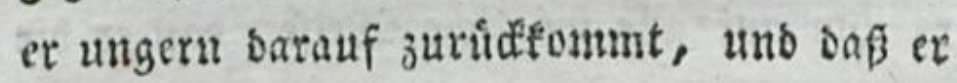
bir jene Geftátoniffe melyr aub bamaligem unwiberfeglideen Drang feines f̧erzens, Das fich) uแm (Finmal égiešen mušte, weil es jut yoll und zu geprest war, als als wollent Singebenbem Butrauten gegen bín that. *) (3)aแb

*) Dies ift nidt felten ber Fall bey Reiteno 


\section{3}

Glaub mir's, er fúblt etu foldheg fajonendes Senchmen imig ontbar, aud ment er bir'b uidyt fagt; er wird bidi) lleben uno sir trauent bis in ben నob, uno Das um fo mehr, wenn bu bann and im Stillen, obne Gieråufh oa

$$
\text { ₹ } 2
$$

fưt

Den. Denn oft ritb ein Reibenber offenberzig gegen unb, nidt, weil er su uns mebr Dertrauen bat, als zu andern ₹reunden; fondern, weil oas Biefílyl feiner Reiden jezt zu einem Girab gefriegen ift, ben er fdlechterbings nid)t mebr allein ertragen fann. Fr muf fich

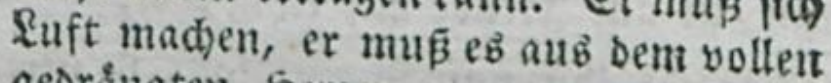
georångten Serjen reg reben, er muई es irgento einem füblenden $2 B e f e n$ fagen, wie unausfprectidich or leibet und fdout lange gelitten bat - forwiege er lảna

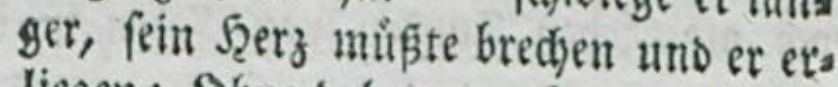
liegen; Dbne baber mul lange zu wábs len unter feinen freunben, fo wirb Der fein sertrauter, ber ibm jezt am nád)? fien ifi, smerften entgegen fommt uns bem er mur Mitgefibl zutrauten fann; gegen jeben Anbern von feinen Freunben wirbe er eben fo offentersig in oer Stunde gewefen fenn; una vielleidit Einen ז̊n, Eine Stunse fpater voer fruber muirbe er gegen Jeben fónels gen - wurde fein Menrch auf oer

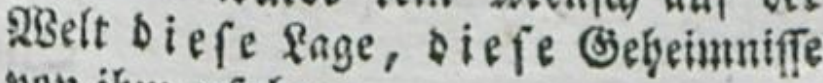
von ify erfabren. 


\section{4}

für ikn benfft uno bandelfi, wo ou für feine Rage denfen unb banbeln fannft.

\section{0.}

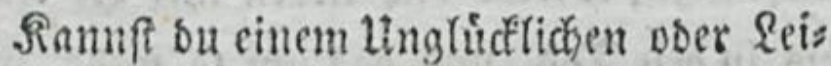
senden irgend eine Frende madben, irgent

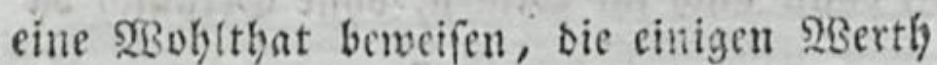
für ifgn hat - o bann fäume feinen 2tugens

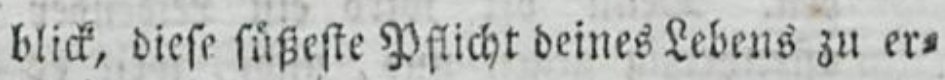
futlen. 2(d) - ber Alnglidelthe glaultit vft in feinen Shrånen: alle fretto foy nun auf

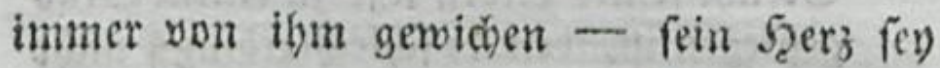
mun fur alle Frente ouf emig toot, - uno bas macht ifin nun fo muthlos, fo verzagt, fo follaff in alfen शerven und fo gelabment in allen Siebeinen. 2fber - wie leidgt ift's Doch sft, jeben Junfen von Reben in ihm aufzuglútyen uno es feinem ganzen 2 sefen fuiblbar ju madjen, onf bud nod greube fur ifhn ba ift! Thätige Secweife Jartlidyer Sfebe uns feiner 2 tufmerffamedit - fiffe Ueberrafoungen - licbesolles alworfommen Kerauggeabueter Bebirfniffe und errathener Sieblingsmuinfoche - o auf taufenbfoltige Ilt tanu man ilym oft wobl, tho eine gute Etunbe 


\section{5}

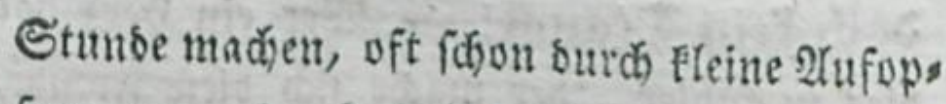
ferungen, ourch tietre Semúbungen, oft f(d)on burd) ein armpliges Stuct (sels, went man's nur auf bie rechte 2 rt ammenbet. Stubiere barauf - eb verlobut fich wobl oer 9) Jube, uno dir felbft bereiteft ou bie feligfte frimmelвfreube. Uno

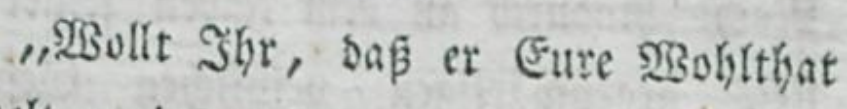
doppelt geniefen foll: fo gebt fie thm, wenn ex eben ermad)t. S luber bicfes (Seftibl gebt Nicbto in ber NBelt. Sch bab' e' empfunden. glüflichen geben, oer nicht mit bem oruiden ben Gebanten an fein Flenb unb mit bem 23 unda)e eines balbigen beffern Loofes, bem erquicfenden Edylafe, oem J̃nterimbbefreyer feiner \{eiden, in bie wohtthatigen Arme fin= fen wirb. Dieb if ber herrichende Gebante feiner Seele am Tage, mo oft fothent ibut ein Traum oab, was er fich beb Tages ver= geblid) exfebnte. SD! einen foldjen Traum auf biefe ober iene Irt gleidfan 3ur Wirf: Iid) gludflide fich fagen fann: Stein! es wat f 3 Dod 


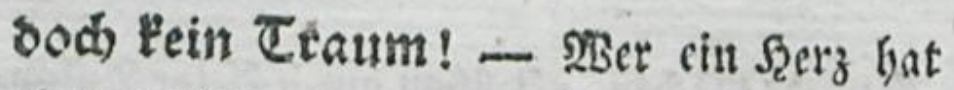
siez zu fútlen, ber mache fich biefe Frrube, und er wiro feltgft die Freube bes 280 blthuns boppelt geniesen. Soer tráumt aud̆ ber Leis benbe ben Traun fénes táglichen Etenbs fort; war ber Sdylaf feine Erquič́nng fur ihn; fpanu feine leibenbe Seele nud bie oft lange

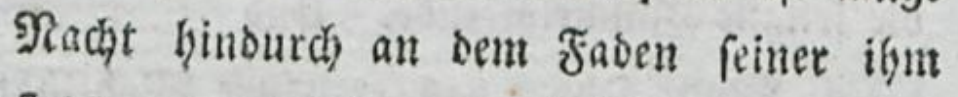
fiets gegenwaintigen peinlichen (sefúble; foblief ex nur, um defto pibvectlid)er 3 ut

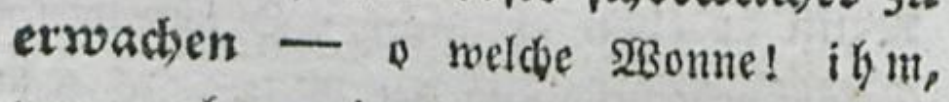
went aud) mur eine Lntevbrechung feines fteto traurigen Joeenganges 3 ll ges wåbren!

Fragt einen Siranten, ber unter bem peinlichften Befübl feiner Edyunergen uno sad) bem (S)enuz einer beilfamen $\mathfrak{A r}_{3}$ nen cin= forlief - fragt ifn, wie ihm zu Mathe war, alb er erwachte, unb fid) mun von 2tllem bes freyet fúblte, was ifn am 2lbeno bes vorigen Bnges folterte - uns er wiro mit Entz̧is

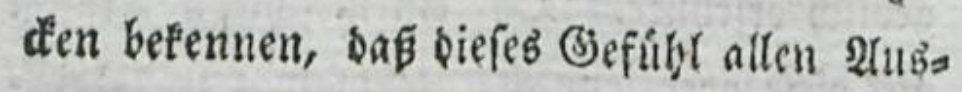
brut úberfteige. Şbm dbnelt $D$ ex felfr, bet lange nach Freuren fdjmadgtete, unb fie 


\section{7}

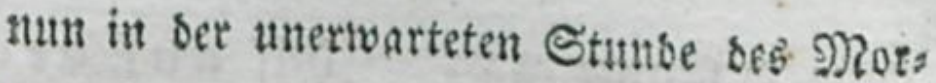
gent fanb.

Unb went es wabr if t, daf wir and

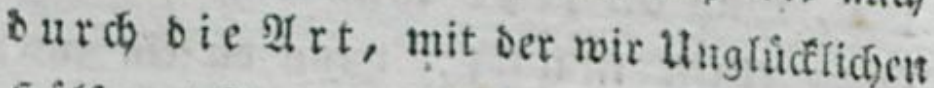
5ृûlfe und Freuben fochenfen, ibre Danlvarfeit gegen unb erbo̊ben, verfeinern, vervol!= sommmen: $2 B e r$ wollte nidit, um sud einer fo esten \&eibenfhaft neue Stoptrafte zu leiben, auf ?ittel finnen, bieb zu berwerf:s ftelligen? S deß Meniden fo febr ver (b) reenes finnlicheb, (j)fiib! fann zu vortrfffidoen 3we: den bingeleitet werben! - Beylåufig! WBie 9) ses Unbanfo brúden, wemn Die, bie etruas furr ibn thaten, fich mebr feinem fein fúb: lenben Serjen anjupaffen verftanden baitz ten! - *)

21.

Raunft ou einem Inglidélidien belfen frebt's iezt in beiner Madyt, ifyn wenigftens Eine \{aft abzunelgmen, Fine Sorge ibm wes niger und fein §eident ifm ertrågliøer zu mas

F 4 den:

*) S. SBciber maditen ilgn weifer uno glứćliç. \&eipz.790. S. 241. 
88

den: fo faume nidt - bilf a uf oct Stelle - verficiebe nidt auf thor: gen, was ou jezt thun Eannft, und je $3 t$ thun muft, wenn bu ein guter Me n f fchen yerbienen willfif.

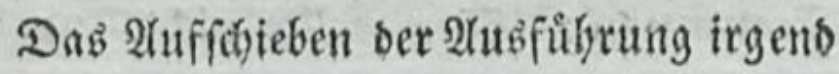

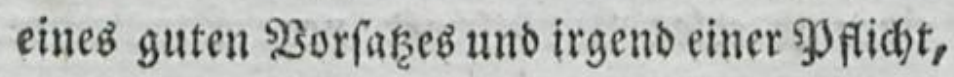
sie meiter feines̆ Hebertegen bebarf, ift jebem Menfchen voll Siraft uno Seele unter allen Utmftånben und in allen 2 tngelegentyeiten uns

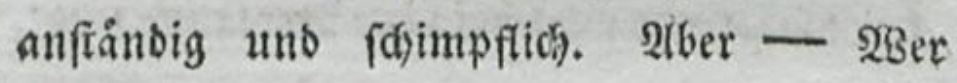

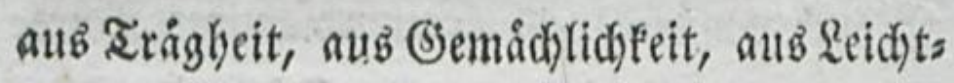

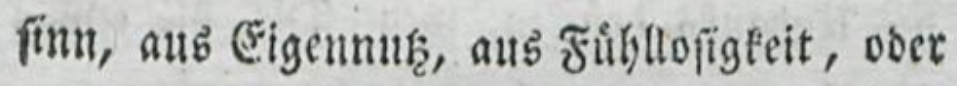
aแt wab für einer uneblen $\mathfrak{U}$ rfach es fent mag, nur Eine Stunbe fäunt, einem htu= glifuctidchen thatig zu belfen, wo es in feiner

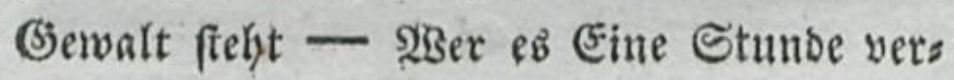

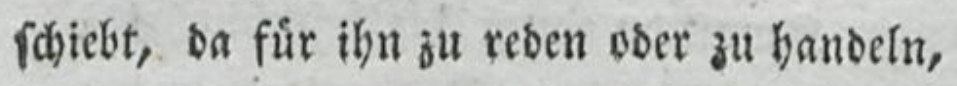
soo er furr ibn reben umb bandeln ann wabrlid, ber ift ein elender, fojlechter Neníd,

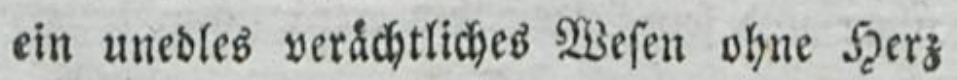
unb ofye Seefe, unwerth aller 2(d)tung, alles âtrautens unb aller \&iebe. 


\section{9}

Sieber - verfengne unb fosinbe beine Menf(ch)eit nicht; braude' Danfbar jebe Srraft uno nưk̨e fonnell jebe (jefegenheit, bie bir bem

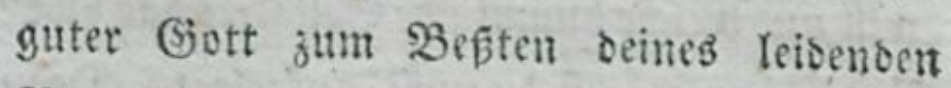
Stuber gibt; trocthe Ilytânen, verminbere Soth, bebe safien weg, frâffe, fegne uns etfreue, olyne Bógern, wemn (Bott bid' wuit: bigt, Dit Nittel uns Belegenlyeit baju in beine Srinbe zu geben. Dente nie: "id bin

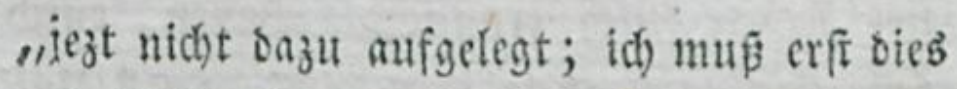

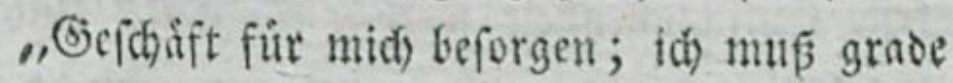
„, beute an biefem \$ergnigen, an biefer \&ufts ,partbie Zheil nefmen - Norgen will ich "f fur ihn arbeiten, ibm beffen fo gut idh

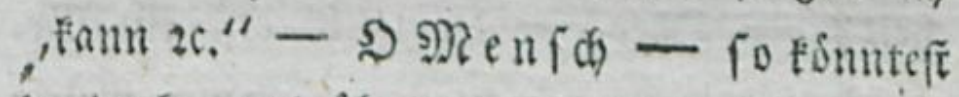
Du benfen uno über beine beiligfte Merifden=

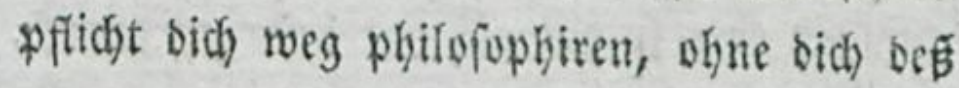

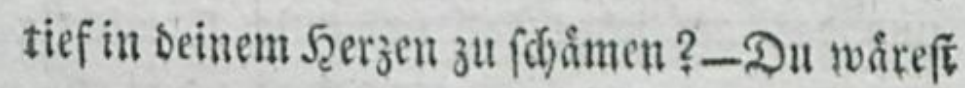
3id)t aufgefegt einem Hnglidfichen zu belfen, no bu ifym bod helfen fannft, fobalo bu nur willft? - פaseif̧t ou, wab oab heift:

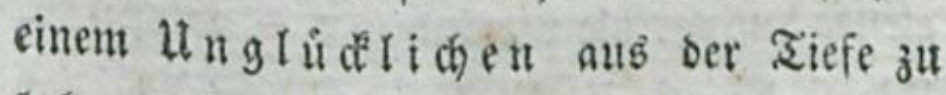
beben, feine Troth zu enben oder zu erleidh= tern, feine agránen bes Edjmerzes und ber

$$
\text { F } 5 \text { 2ngft }
$$




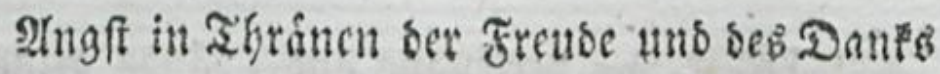
ou vermandeln - ijm ein Engel won Bott gefandt zu fenn - weist ou wab ong fagen will uno welde bimmlifare Geligfscit bag gibt? uno Dazu wareft bu nitht nufgelegt? Dazu fhiene bir jese 2 rnfrengung, iebe Pleine Infs opfertung zu groß̧? Das wáre nidt menig=

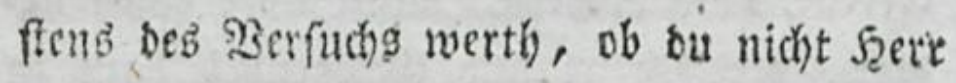
beiner \&aume, beiner serfitmmung uno beis ner Rrafte surch crnftlidę șoflen, ourds fefe Entfdioffenbeit werben fonnteft? linb

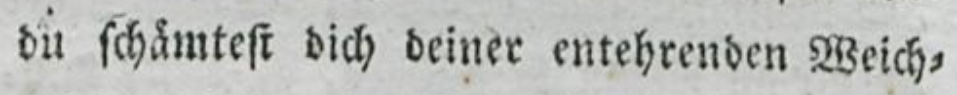
ridfeit nicht, bie Mebr liber bich vermag,

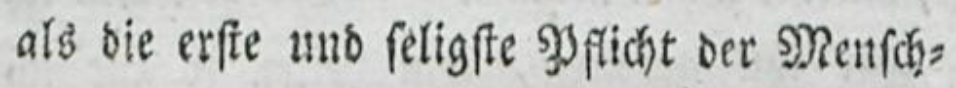
beit? - Soer - ou willft exft ein Gies

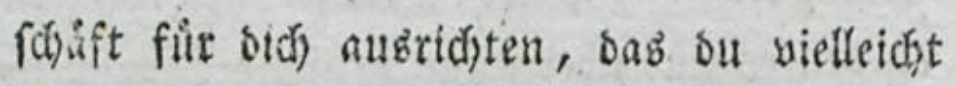
morgen eben fo gut als jezt bejorgen fannf, 4no bab auf jesen Fall fámerliḑ fo wichtig if , alb bas, wns bu bem Reibenden fchulbig Fift? - Dber - bu willft lieber ben lln= gluxutidjen länger bulfflos laffen, nis cine elenbe \&ufipartbie verfăumen? - $\$$ pfui! SBarft ou nie in श2oth, ober fanmit ou did in bie Lage eines Notgleibenden gar nidht.

bine 
Gimeinbenfen, Gincinfúgten, und Famnf ou's

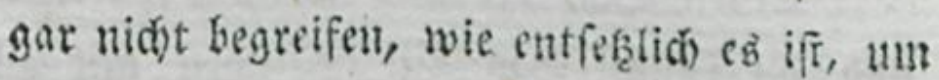
folder Urfach willen einen Unglidélió) mur cinen 2lugenblicá tánger sem marternben

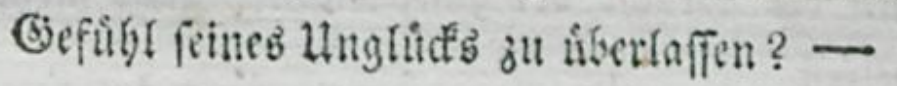

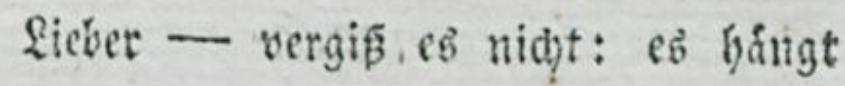
oft fo ganz yon Umfintinden ab, cinem \&eis Denten belfon zu fo nuen, unb birfe llms ftande treffen fo folten jufammen, unb wed) fefn bann wieber oft fo unermartet uno fdjucll, uno an ifgre Stelle treten wieber ants bre ein, bie uns dann jede fentfe fo unglaulbs

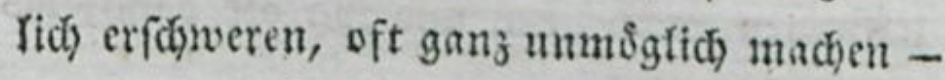
onj es in biefer frinjidit jur doppeiten $3 y$ flidit wiro, jebe (jelegenfeit unto jeses 9Rittel a uf ber Stelle zu ergreifen, wo fie unb ento gegen fommt ober wo es̉ zu erreichen iff. -

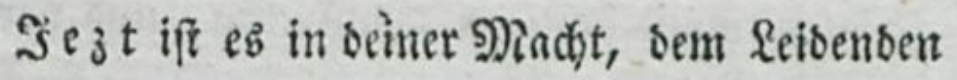
wirffam zu helfen; gebrauche bies §e zt, was bu baft - verfhtebe S2ichts auf eine Seit, bie bu nod) nidgt baft, uno úber bie uns Deren umfténbe bu nur felten Jacre bift, bie vielmebr faft immer fiber bid) foerr fint. -

Eี่กตะ 
Einen నag, oft Eine Stunbe frater uno bu fammit bem lunglicáliç)en mit alt beinem SBiflen, mit all beiner Macht, und oft mit ber Macht ber ganjen $2 B e f t$ nidjt melye belfert.

122.

Entferne von bem Seibenben alle reere Şifitenmacher unb alle fabe Eonodentigefich.

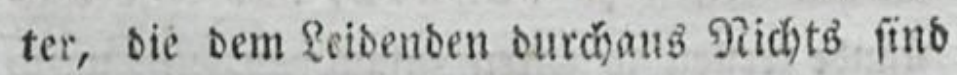

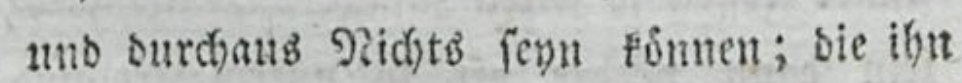
burd ihr "mon cher ich contonlire" zu troffen,

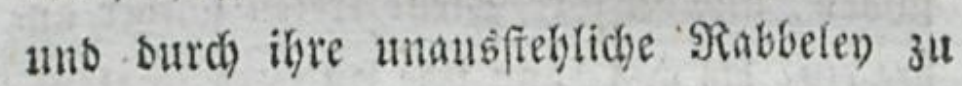
zerftretten vermenten und ihn oft bamit bis zur ßaferen bringen.

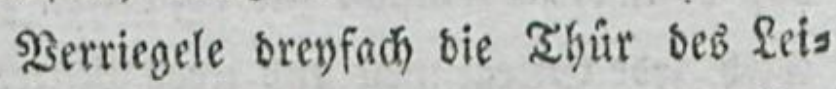
benten yor allen Slagetweibern, vor allen f(d) woigenden Frau Saafen, yor allen fcinfas ferigten monofübtigen Coufinden, uno vor allen beiligen fupergottfeligen Grostanten.

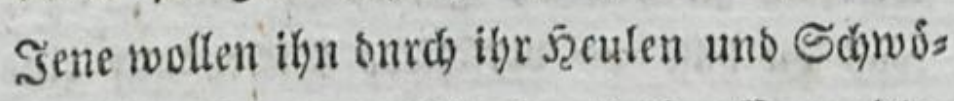
gen uno Betergefreifoch, ourch iffe (sonvulfios nen uno Shnmachten, burd ibre wilben, Gimmelfúrmenden Giebehrben befänftigen -

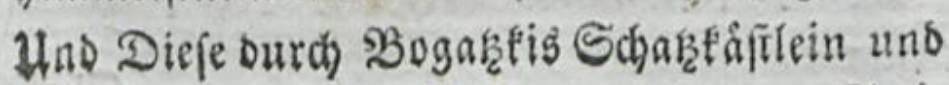

Scris 
Soriverg Seelenโdan alle peine Sdymerzen fitlen, uno atle treiben ihn bamit bis zur פ̧cr3meifelung.

$$
23 .
$$

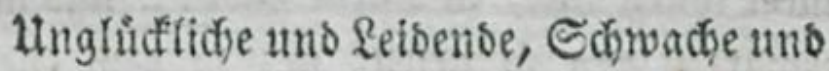
Srante find leidgt zu beleibigen uno ju frón fen. Der geringfere felbf fdeinbare Nanget

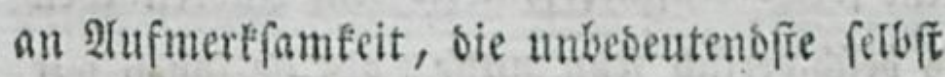

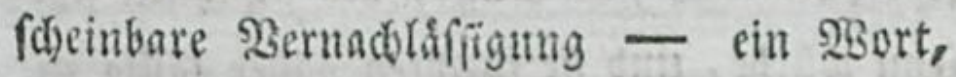

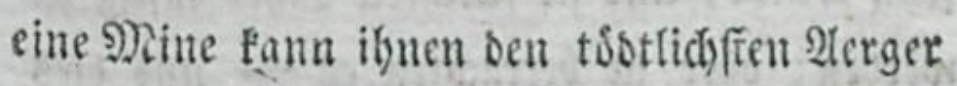
verurfachen. Reiben uns Sdjmerz surchfäurent oft fo ifren Eharacter, dof fie felbft oas 230 ort ber treneften \&iebe oft nidgt verffehent tum foilef auffaffen.

Sie yerlangen als Reibenbe oft ein nusfdylie=

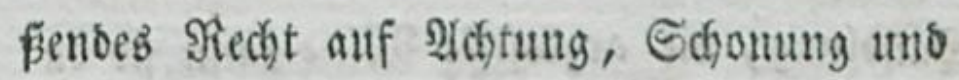

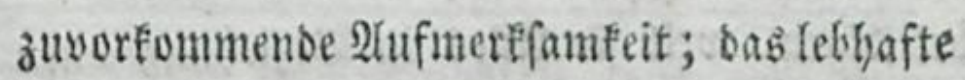

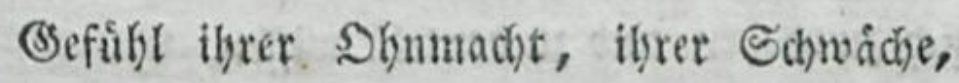
ihrer Şutffobbedirftigfeit macht fie forberno; fie betrachten es als bie umeingefdrånetefte S(d) alf fie merte, alle ibre Beourfniffe ifnen abs

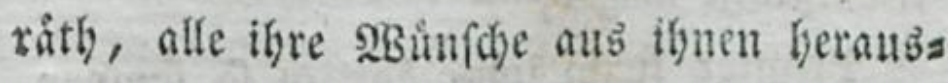
abuet uno fie alf ber Stelle befriedigt. -

Bes 
Bemerfen fie bufer, ober glauben fie's mur ju bemerfen, daß̃ man einmal láfing if in ber föแtbigen 2 tubibung biefer \$fict)tu, fiegt man ihnen nicht in Allem juvor, wie fie ez erwarten - fo gefellt fid ju bem (jefífl ifrer Dhumadt leidgt bie Sorffellung, Daß eine folche 2lufmerffamfeit bem Shbern ebent

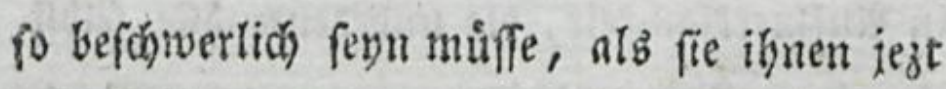
feyn wurbe, uno fie furthten bafjer láftig zut werben; oder fie betrachten $e^{b}$ - uno bies Hod) Sfterer - grabebiu alb \$angel att Sicbe, glauben fich mun leid)t iberall vernach: Iåkigt, finsen felbft in den thåtigfren $\mathfrak{B}$ erveis

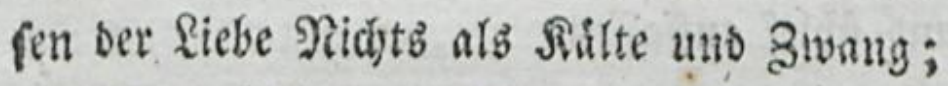

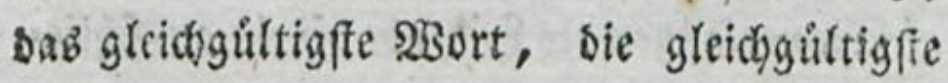
Szandlung, bie arglofefte Mine tann fie da beteibigen, weil fie 2llfes in Beziefung auf (iid) зu breben und zu beuteln wiffen, *)

Dazu

*) Omnes, quibus res funt minus fecundac, magis funt nefcio quomodo Sufpicioji, ad contumeliam omnia accipiunt magis;

Propter fuam impotentiam fe femper credunt negligi。

Terent. Adelph, act, 4. Scen. 3. 
Daju fommt Dann, safalle ibre ఇerven teizbarer, fủblbarer, empfintlicher fint ; fie

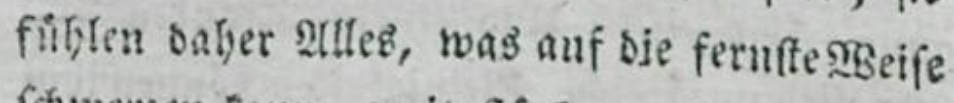
f(d)merzen fann, weit frâreer unb peinfidger, unt bie getingfte unfanfte Berúbrung fanı

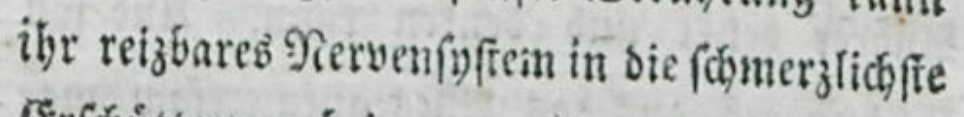

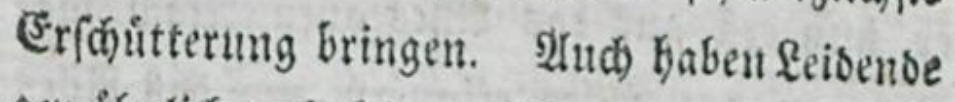
gewoigntid) weit feinern Simn uns weit feiners

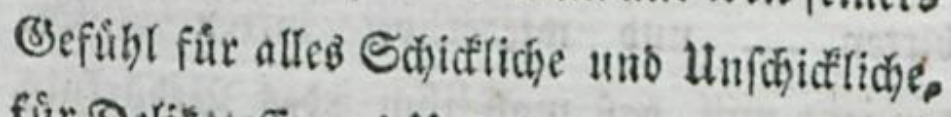

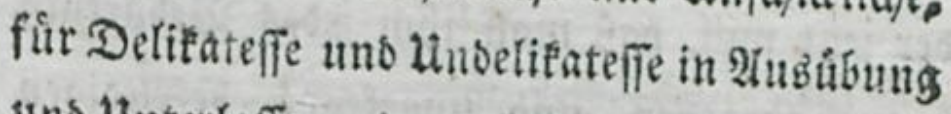

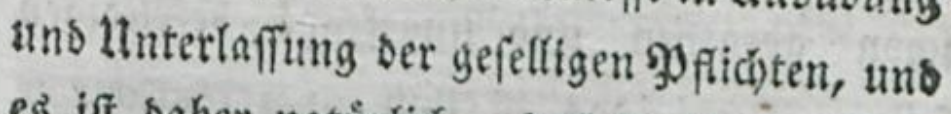

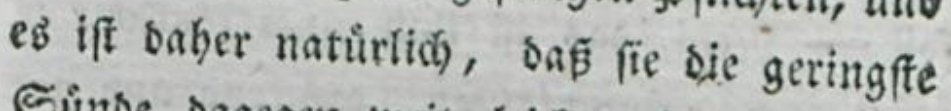
Sunbe Dagegen weit leidter bemerten uns seit empfintlidger aufnebmen, als jebet Intoere.

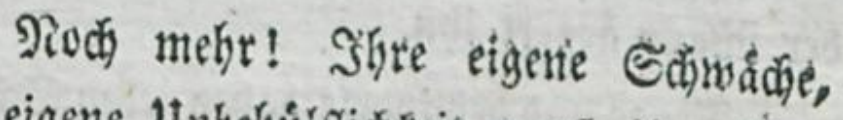

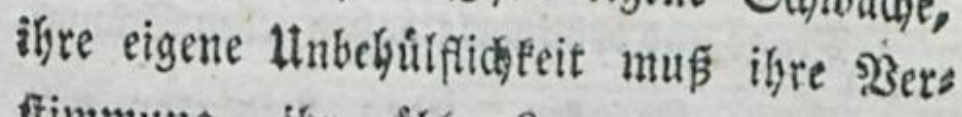
finmung, ifre tible fanne noch un eitt

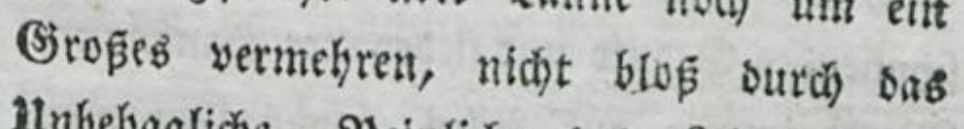
Anbehagliche, झुeinliche bes Leibenb, ber siranflyeit - fonbern aud burdy ons bes

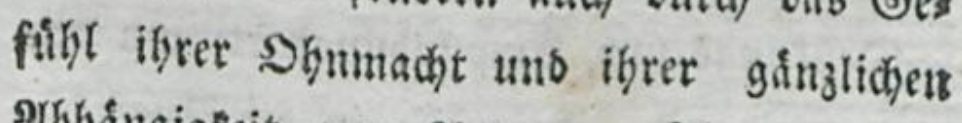

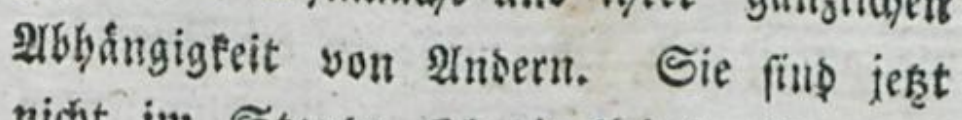
ricit in Stande, fid bie fleinfte sequems

Iings 


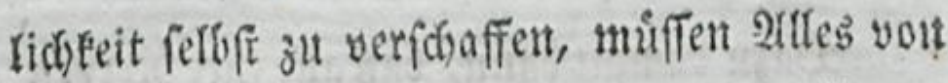
2lnbern baben unb immer 2 (ndere in SBewes gung bringen. Itno einem Sranten wie viel unzăblidge Rleinigteiten fómen ihnt lafftig ober unbehaglidh weroen, an bie fein 2nbrer benft uno denfen fann. Uno foll er sun erf auf bns 2 glles aufmerffain mas

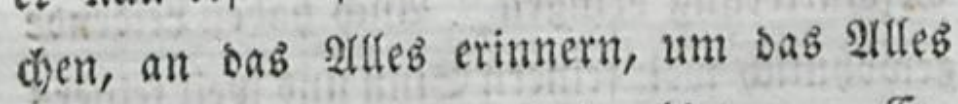
bitten - uno wieberfolt bitten - Er, Der jeşt will, oás man ihm jedes Sedourfnis

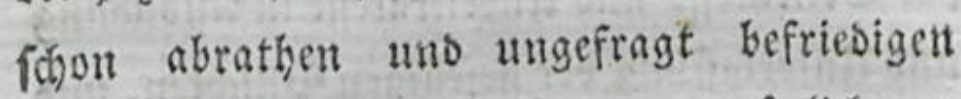
foll - Nein, bab iff if̧m unertriglict) ; er fiblt fich vernachlafifigt, orictent uno ge, sritift, fich uno 2lnoern jur $\mathfrak{E} a$ fr, uno nut madit's ifm Sieiner recht und bie Fliege an ser 13 and árgert ifrt.

Trage uns behandele baher jeben Sămas (j)en, jesen Siranten, jeben \&eibenten mit faronenber Geduld und ficbevoller Sanftmuth. Siert' immer auf 2llles, was ifm woht und was ihan wely madst; ftudiere feine gans ze. Denfungs uno Entpfitoungsart; um ihm nie auf irgeno eine weife in bie Dateer ou Fommen; fíble Didy ganz in feine jefige 
Lage binein, um alle feine 2 simfide uno sgedurfuiffe ihm abzulaujhen; werbe nie lairiig, nie unfreunolid) mit (5iner Mine

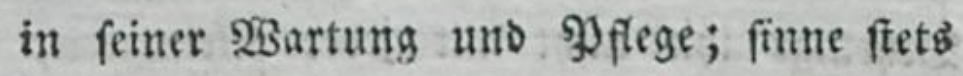
Darauf, ibm iese frulffeiffung fo zu Yeiften, wie er's am liebfen hat, unb madi' ihm ies

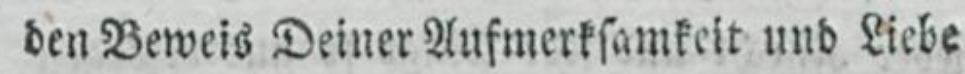
surd) sie 2trt, wie Du ihn gibft, nod) wohls thătiger uns erquidender.

24.

Şuite Didb, dem Reibenben, naç Irt

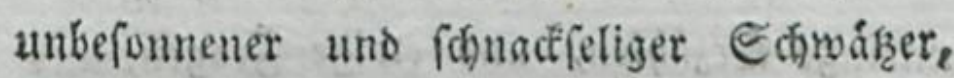
burd) (jefpråde unterbalten zu wollen, die burd)aus fein sntereffe fur ign haben. F̧ute Did), utangenehme Erimuerungen ohne

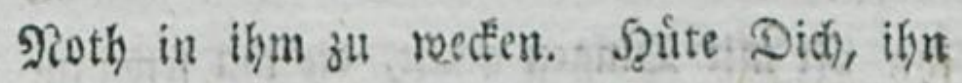

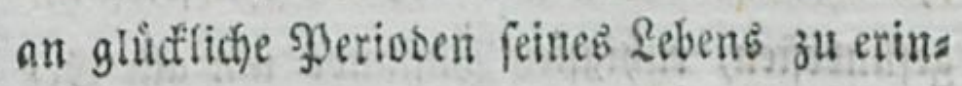
nern, bie er burd fein gegenwártigeb \&eiden verloren bat. Shartere ign nicht mit פes fchreibungen yon \&ufpartfieen uno \$erguน⿰ gungen, woran er jeķt gar nid)t Theil nel)= men Eaun uno niag, wovon fich fein ganzes 2iBefen iekzt wegmentet. Rapportire ifm

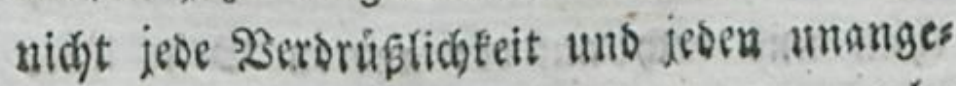
(5) nebs 


\section{8}

nelgmen şorfall ats feiner Familie uns aus feinem ber Cadje abzutgerfen oder fie za ándern. Sebellige ifhn niót mit feber Riteinigetit, bie in Şaubwefen vorgenommen werben foll, bie fich oft ganz vou felbft verffert, tho bie feber Sinecht und iese Sago fo gut keforgen fann, alb er felbft. Utud dente' immer baralt, wie peintich uno unangenely $D$ it in Deis nem gefunben teidentofen allfande bas cmige Eronaten, bas unbefonene Erimuern, unb unfluge Erłáblen, uno ewige fragen, uno ewige Sarfen tno Renmen feyn wirbe. — Es iff entfergelich, wie wenig Sinn uno Oefufhr man oft mit bem\&eibenben Gat, wie man ihn oft mit Dingen quâft, bie ign futbfr int gefundeffen, ruhigfen Buftande un alle (bes ธutb unb um affe Sernunft Gringen múfen! 25.

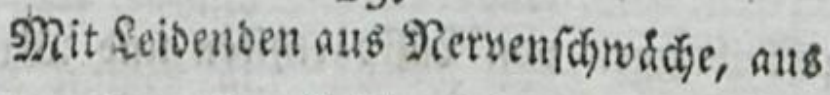
ůberfpamuter Empfindfamfeit, mit hyfferis fden Danten, if áufferff fobwer umzugehen, wenn man fie nidjt frimten, ifnen nidjt wely thun who body auf ber ambern Seite ifrer Sirants: 
Rrantheit feme netue Nabrung geben will. Sie verbienen cines Theils fo viel Schonung, fo. viel imnigeb Mitleio, fo viel Erteid)terung, fo viel besuld und to viel feine 2 ufmert's famécit, und bod) barf man andern afjeils oft to wenig Sdjomung ifhen berweifen, fo wenig einftimmen in ifgre Rlagen, fo wenig billigen ifre Ifgranen, fo wenig ifyen gebent yon Dem, wab̉ fie forbern und wab ifnen

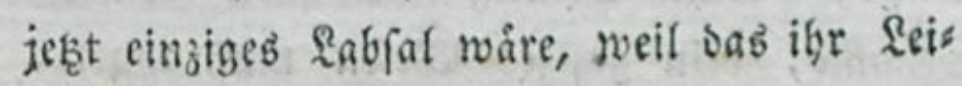
ben mur unendlich vermefiren uns balb ganz unbeilbar machen wároe.

Man leibet entfeg̨lich bey folden Rran= fen; man fuiflt immer ihr gegenwártiges Eleno, man migte es ifynen fo gern erleide" tern, yerfififen - uns sod friblt man fo

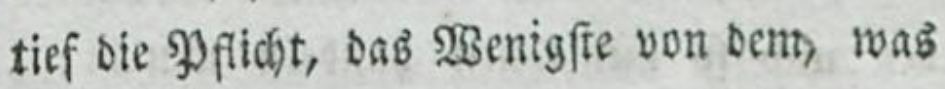
es if)nen jeşt erfeichtern witrbe, anjutwenben, ween man nidjt Hebel árger maç)en will. ङ゙B̈ gebort baber ein feinfúblenber aber ges ferbter (Sharafter baju, ber fie behanbeln wille wie fie behanbelt werben miffen; alle mar:

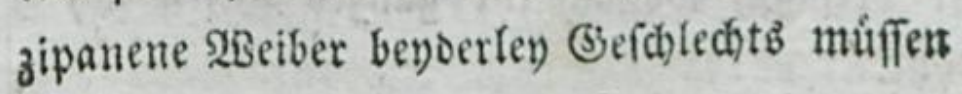
fidledterdings von ihnen entfernt werben.

(j) 2 Şaft 
I00

Feaft Du cinmal ons Butrautu foldber Seibenden unb einige 2lutoritát ůber fie ges wounen (bas bailt nber oft aufferft fóbmer, if Dir aber fdyled)terbings notbmendig, wenn alle beine Bemifungen nicht umfenfternn, niajt verfebrt wirfen follen) fo forich oft mámulidy zu ignen, fuche jebe Siraft in ibnen zu wedten, bie ibnen noch ưbrig blieb. Deine S\&lice, beine Simen, Der Ton beiner Stimme, bein ganjes Sisefen mufibnen aber Dein berzlidjes Mitgefulgr berweifen,

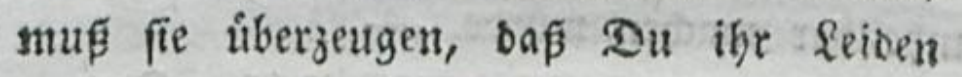

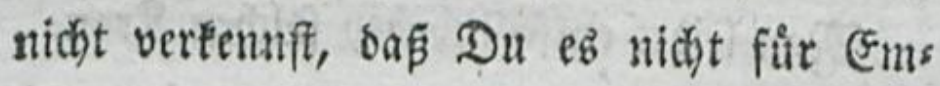
p finbeley, nicht für eingebilbetes fonbern fur songreb \&eiden biftft; oaj Du aber alb ware mer theilnebmenter Frenno fo mit ifnen resen uno fo mit ifyen bandeln in üt; baß̧ es baker nidyt Sirnufamteit, Ralte uno Unempfindicbfeit fondern bie treuefte Freunds fdift iff, wenn Du nidjt immer in ibre Singen einftimmefi uno wenn Du ifnen oft verfagf, monacis ibr ganjeb sgefen fich rebint.

Rebe 


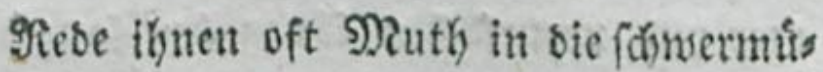
thige Secle und fudge ihre Sygantafie uns merefliç) auf beitere Biegenfínbe zu lenter.

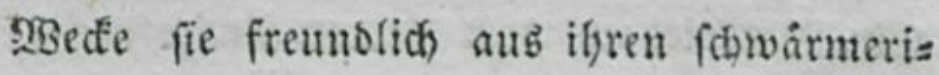
foyen Tråmen unt leite iffren Blict auf bie

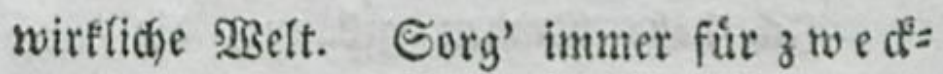
mán bem Tete - a - Tete mit fiá) felbft, nie ber Einfamécit, ber Ettille, ber Dảms ntertug, ber Dunfellecit uno bem Sponts forein. Entferne fie son allen angreifenden Scenen ber Frente wie ber Traurigfeit. Bewalire fie wor allen beftigen Reibenfhaf: ten. Lltio - vor allen Dingen: wende

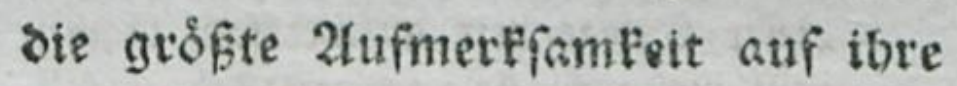
Lecture. 2unbaltend uns allein burten fie nie lefen, burchaub aber feine Şidjer, bie

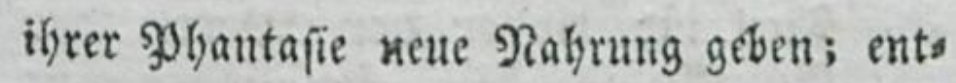
reiß ihnen baher unerbittlid), zum 230 bl ih= res Leibes uno ifter Seele, alle Romane, alle Edauppiele uno jede zacile, bie yon Empfinofumfeit buftet; fie fino árger, zekn= mal árger für fie, als baz árgfte bift, benn fie lábmen ifre ganje. Seele auf im=

mer 


\section{IO2}

mer uns berurfaden ifnen ben langfamfien martersollfien Zos. Dap bies die ftrengfte SBalyrbeit iff, bavon zengt leiber mely alo Ein Deyfpiel aus dem Fleinen Sreife meiner Defamuticjaft. -

S(5) babe Damen gefannt, bie butch ifr unbånbiges Romantefen fich um alle (3)es funoheit beb \&eibes uno ber Seele bracbten uno burch ifyr unbándiges Romanlefen ifre unfelige Siranflyeit ganz unbel(bar mad)ten. *)

Sić

*) Sţie dab mogliá fen, liebe Leferinn? D leren uno beberzigen Gie nur for= gente Stelle aus einer nufferf empfeh: lenzwerthen Edyrift, auf bie ich Sie ben biefer Gielegenbeit aufmerffam madber will, wenn Sie fie nod nid) temuen folts ten, unb bie jeşt mebr wie ic gelefen und beberzigt ju werden verbient; ibr Sitel ifr: Ueber den werth oer Empfinofampeit befonders in Zitclifiot auf oie Rom ane 2c. Salle I786. Sie jeigt auf die treffendfe,

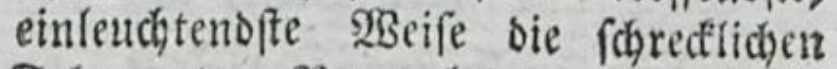
Folgen ber Somanleferen auf Şerz, Geift uno Seben. Sh bebe bier nue folgende Stelle aub, oie idb bier anfühs ren wirbe, wem fie aud nod) wenis gex bieber gebirte. So etros fann jent 
Sie weinten und flagten ben Sage und bey গad)t, ofyne zu wiffeu, worifber? Seine Frenbe fam melgr in ifyr $\mathcal{S}_{2} \mathrm{er}_{3}$ unb feitt

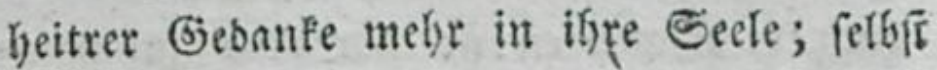
bie ganze grofe Natur in ifyrer fdosuffen Serrlidgeit gatte feinen Zroft fur fie, nur

शaks

jest nidit oft genug gefagt werben,

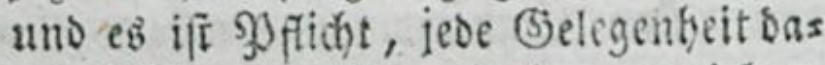
ju felbfi gewaltfam berben zu zieben.

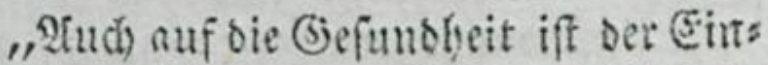

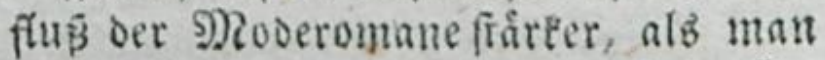

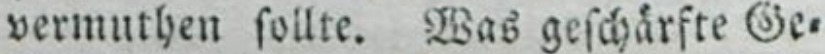
(d) lecht tempfindungen uno \&eidenf(haf:

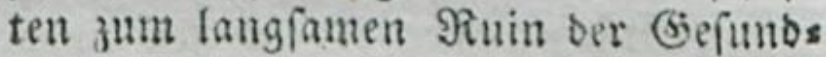
beit beytragen, iff gar zul bekannt, alb

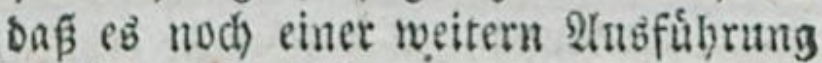
bedirfte, 2luffallenter und ooch zhgleid weniger bemertet find einige anbere ${ }^{2} \mathrm{r}=$ fcheinungen, bie fich unter unfern ro= mantifden f̧elden und Fetsmmen ereiga nten.

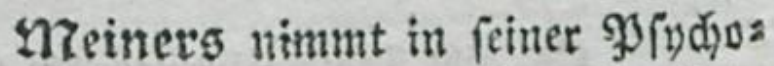
Togie fúr bie Bafiß ber Smagination, Sinnlideseit, Empfindlid)teit, Empfinds fameeit ant. Jam wiberfę̧t fich 3lwar Cidemann mit verfáiebenen Bruins ben, uns behauptet, bafi er viel jt

(5) 4 WES 


\section{4}

Nabrung für Sdywernuth unb Thránen. Snmmer angfflid, immer mutblos, immer verzagt, ofne die fleinfie Urfach angeben zu formen, fdauterten fie fdredtid) zufummen bey bem Rauffenen jebes S3aums, bey bem Sifpeln jebeb 2 lattä; jeber soogel ber un,

\section{ers}

wenig fage. 2lber genug! S'n bem ers fien Empfinsungobermogen licgt ooch ber erfie, wefentlichite Urftof ber Ein= biloungsfraft, uno bas suffere $5 \mathrm{~m}=$ pfindungsvermogen nus ilyr bie erften

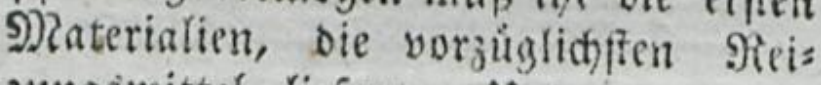
zungentittel liefern. Ituo wemn alfo

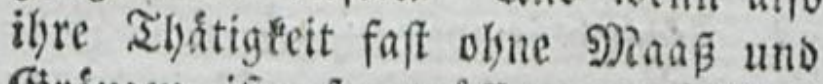
Granjen iff; fo muffen natirlidyer SBeife bie Perven ourch bie ftete $21 \mathrm{n}=$ ftrengung, in weldber fie erbalten mers Den, zu einem Grade you Feinbsit uno Edbwidhe berabinten (oder foll id) fins gen, erbjbet merben?) weldie fafi feinem einzigen áuffern cinbrud 20 berftans leiften fann.

EB gibt Frautenzimmer, sie foron von शatur kufferft leid)t zu Shrånen be: megt werber fonnen. Man onrf aud

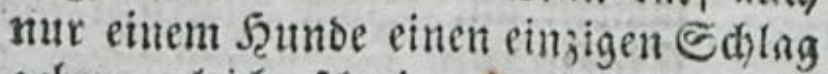
geben, gleid fobmimmt ibr 2 luge in รbrånen. Ein untrugglider SBeweis 
erivartet yor ifnen anffiog, warf fie in Dhus madbt; bey jedem bitren Saum und ben jeben verwelften Slimçen frúrgten ifnnert Thránen aus ben 2lugen uno laute filagen yun ben Sippen; jeber fiarte Tritt auf ben Buben, jebes Bufdlagen oer Thur, jebes ลu=

ber anfferfen शervenfeinbeiten uno Berbåltuiffe! DBem num oiefer ఇatur: febler nod) ourd) bie Simf oer গios manbidter erbofht wiro, fo miffen noth: wendig alle oie Rrantbeiten entfeben, meldhe in oer Sdbwadde uno Reizbars Peit oer פierven ifren. Sruno baben. -

Whie (đ)wåd)lid) ift nicbt ber (3e= funtheitรzวuftand einer empfinbelnben

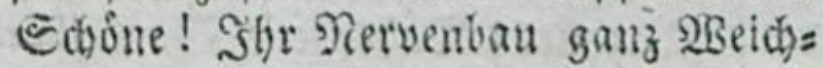
beit und (Empfindlidject für die Eins orudce ber 2 luffentwelt! Illes, fogar bie Seranderungen ber Iuft uno Des Rlima wirten auf fie, uno die Reaction ifgrer Empfinbungsorgane frebt mit biefer

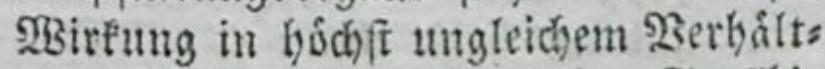
niß. Nidbt feltent gránzt ihre Senpibi= litat an bie Senjibilität mand)er Thierars ten, welde die sierainderungen ber \&uft Innge vorempfinden, unb, wenn fie unter einen anbern f̧immel den, langfam oabin fomad)ten unofierben

(5) $5 \quad$ In 
3ufaumenflirten ber Deller uno jesez 3us fammentlingen ber sbáfer verurfad)te ifnen-

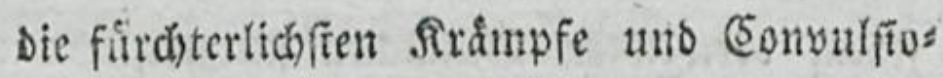

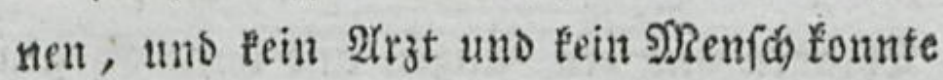
ijnen berfen. - Ben Gott! id tibertreibe

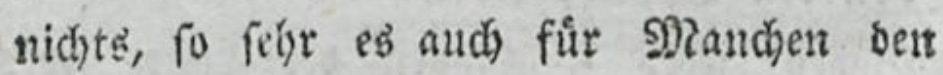
Eḑein baben mag. -

\section{Uno}

- In iebem Romente ifrea Rebent fúblt fie oie Dispofition ilyres Sírperb.

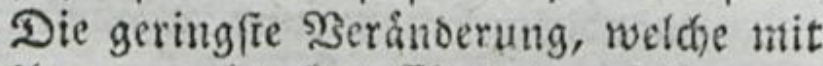
ihm vorgeht, hat Einflus auf ibr gans

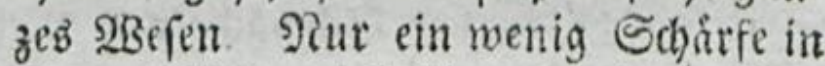

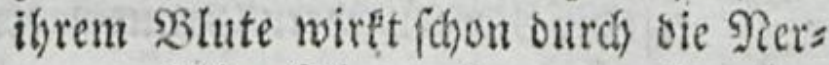
yen auf ifgre Sobantafie, uno gibt allent Bilbern berfelben cinen tribuen, metans dolifhen -2nftrich. Daber alfo iene

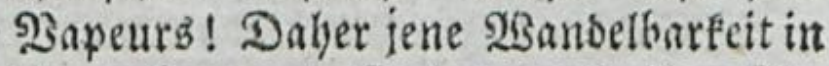
ibren Launen! Dalser aud ihre Fies berhaftigeteit ! Denn bie thierifde શatur befrrebt fidh allemal, biefe Schårfe, ober was eร̉ auch fúr eine frembe uno (ch)ads bafte Materie fey, wegzutreiben uno bas beist eben: es entifeben $F^{2}$ ies ber. Gelingt es ifyr aber in oiefem Seftreben nicht, fo werben bie Rerven immer f́chwádher uno reizbarer, unto $\mathrm{e}$ exfolgt Dab, was bie 2lerzte ben Fie: bercharafter nenment. - Eben oiefer ho=

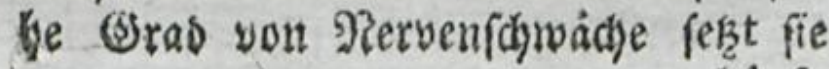
ได่uf์ 
Und sas atles wax folge iftert unbinsigen unferigen Riomanlepe: rey! - SBBer Sbren bat zu bóren, Der Góre!

\section{SBillfte}

biaffigen $D$ bn maditen aus. SRs= gen immerbin biefe, Dlymadoten zuwci= Ien aud blope Derftellung fonn! Der Siegel nads grimoen fie fids auf ibre Rórperbifpofition, weidbe jum Ţbeit

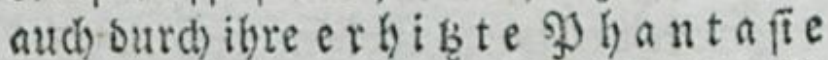
befimut wird. Der nidbtbeseutenofre Lumftand, welcher Beziebung auf ihr Eelbft unb auf ibre Irage hat, ift ibr auffalleno, uno treibt oft ibre झुbantas

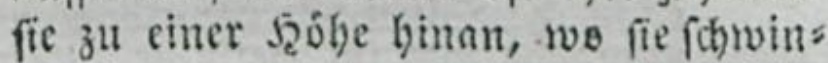
Deln, wo fie $\partial a b$ Giteichgerwid)t ihrer

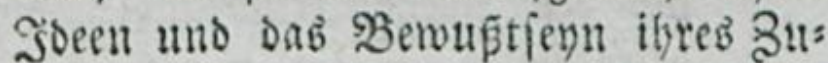
ftanbes verlieren mus. Sie if bald yor Freube, bald yor Setrubní, bald yor Sdirecten auffer fidi); uno wenn nat fie nidht nad) uno nad) zu Diefen (5m: pfinoniffen vorbereitet, fonbern fie wie mit einem Sdlage in \&ufrulyr bringt, fo fann felbft ber Too die Folge das yon (eyn. ") - 2ud fogar im Sd) (a=

*) Sn einer anfefunlichen Stabt, wo man= d)e Tisbter Romane uno Sd)auppiele lefen, rábrend baß die Natter fpins 


\section{8}

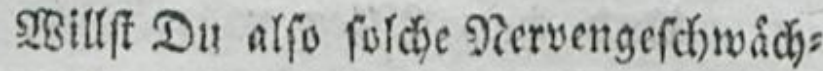
te nicht rettungslos unglicflich twachen, fo

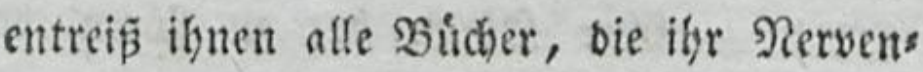
fiffem nod melyr f(b) ad ben, noch feiner, ans fpreacenoer, reizbarer madyen.

IBola

fe mad)t bie geheimfte fórperliăe (F) pfinoung ibre 5jbantafic rege, uno biefe malt alb̉baum zur Dantbarteit die Em= pfintung mebr als zulfelgr aus, uno reibet lange ofolgen von anbern Joeen uno Empfinoungen an fie an. Selten

ge:

nen ober sie Rliche beforgen, wutbe yot jroey) Monaten ein Jranenzimmer bes Morgens todt im Bette gefunton, bas fich bes slbends frifch und gefund nies gelegt batte. Eie hatte benfelben grbens ein rublendes Schaufpiel auffubren fes ben, unb zwar th Gefellfchaft ibres SBråutigamb, ben fie nicht leiben fonns te, aber ju beffen Fertath fie Tiemand zwang; uno furz zuror batte fie an zroo झुerfonen, die ifr tieb waren, in foldhen Iltsorud en gefdrieben, weldhe ibre bisherige \&ecture febr beutlid ver: rietben. S. Deutfobe Jeitung 1784. St. 40. Uleberhaupr lefe man $\mathbb{C}$ if o t Wert von den Ireventrantbei= teil vornebmex und reider Per= forten. 
SBollen fie und miffen fie surchans le: fen, fo gib ihnen ben Don Cutrote, Des rcgrine Pidel, Eiegfries von Linien. berg, (5efdicte der walobeime, Le Eage's uno Ecarron's Liomane, bu= Sibtas, 23Iumauev's, 2leneide, Langs beino Gesidhte unb oiefen ábnlidge Sd)rif= tert. geniefit fie einen ganz traumlofen Sdylaf. Der boch allein von wabrer Etíreung if ; uno im Tranme wird fie von eben Denfefben Reidenfó)nften befrurmt, be= nen fie in wadienten Suftanbe fo yier Semalt über fich cinrdumt. Tut im Beitmañe find ibre Sråmme youn wirt"= lider Siaferey unterfdieben. Hno bie= fer Sinferey nabert fie fich ourd) Dab 2lles mit vollen Sdritten.

Dod) es ift wol ưberflufing meiter inb Detail ju gehen! - Echun Die Ein: formigteit, oie 9)?onotunie in allen $23 \mathrm{er}$ richtungen Des romantifchen Echwár= mers fogeint feine (s) funobeit mad) unto nad zu untergraben. Sft verbreitet fid) liber feinen ganjen Buftano cine trautige Unbebaglid)teit, obue bap er ibre Urfach, ibren eigentlichen Sis bes fimt angeben fúmte. Hno nid)t fels tent mogt' er lieber biefe Hnbebaglichs teit 
ten. Seß̧e ihnen nux furje Termine, it benen fie lefen Dúrfen; lap fie wo mỏglids

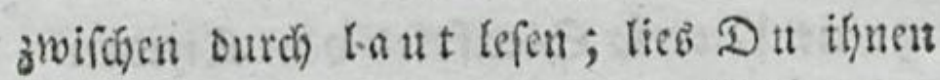
Sfterer yor, uno läp babey fein 9) Mittel nn= verfuct), was auf fie roirfen uno igneneine beitere Stimmung geben tann.

$23 e s$

Feit mit einer wirffidyen Sirantheit, ja wol gar mit bem Iube verwedfelt fe= ben. So unangenefym if fie! 2lber rober fommt's? Dic Drgane feiner Sुgantafie uno Empfinoungstrnft wer: sen burch Die ununterbrodine 23 irffam: feit, in oer or fie erbalt, allmablich abs: genub̧t uno aufgerieben. Indo biefes ziebt alboann umbermerft ben limfurs fetuer ganjen Mafdine nadh fich. *)

*) Nelgr liber sie SBirfungen ber cin: biloungstraft auf oen Rorper f. . $\mathfrak{Z}$. ber Tieoenann Luteriuch. Liber bent

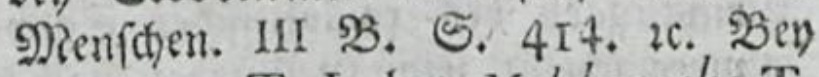
Montagne T. I. bey Malebranche T. $I$ liv. 2. P. I-3. in bem Sanobuche fiiv Leute, oie Eetne 2levite find, Th. 1. Hbfon. 6. in 3onnets 2lna= Iytifityem Derfud tiber ote See lentriffte. Souptff. XIV. XX. XXII. u. a. $m$. 
33efadsfitige fic fo viel alb moglid), bring fie in f stperfiche Tyatigfeit, bey

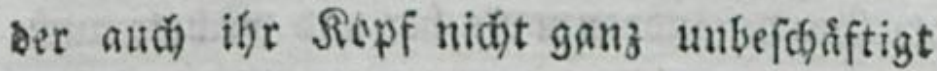
bteibt. Ueberlafi thnen nicht immer die SBabl, fondern trag' D u ifnen oft ein (jes

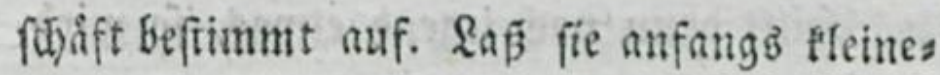
$r$, weniger anftrengende ? 2 rbeiten verrid)ten; Ia je fie immer babey einen gemiffen befrimms ten Broéc yor 2lugen baben; trag bant uns yermertet arfes bazu ben, baß fie ihnen ges lingen miffen - Das medt ihnen ummert= Iicb) Frettbe unb शuth - geh sann alfs mäbliá ju fínwerern fort $2 c$.

Fubre fie oft ins freye Felo, laff fie Berge erflimmen, oic nid)t ju anftrengens fur fie zu erfégen fins. SBanbere mit illen oft ju Freunben ůber Feld, ober nach andern Srten, wo fie nod einenbefiumten Sebens zrvect erreichen, ben fie inb aluge faffen ển nen. 2ring fie liberbaupt oft unter beitere Freunbe, bie fie lieben uns bie son ifnen geliebt merben. Bedfe ifgre tráumende Sees Ie ourds bie glles belebenden פZittel -

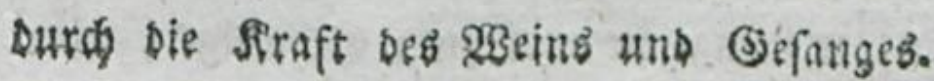
$\mathfrak{E a f}$ 


\section{II2}

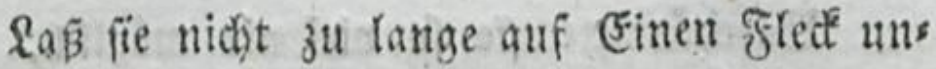
thatig fiscen; verwedsfole oft die Ecene uns. die (Seginftande um fie ber uno wechste aud immer in Doiner Manier ab, mit ber Du fie behandelf́t.

filft bann nod) irgens etwas, fo wirs bieb, unter Şottes uno eines vernunftigen 2(rzteb SEeyftano, noch bey ifnen berfen.

$$
26 .
$$

Menfdyen, die zu Leiben nur affectis

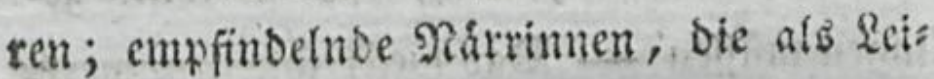
bende mehr intereffiren wollen, unb barum bie Rolle frommer Dulderinnen आI fpielen fudjen - Soldie verdienen nur Bleidgails. tigreit, Spott uno Nerachtung, Uno fie pflegen aud gar balb von ibrer 2tbernbeit gebeilt zu merben, fo bald man mur gar teine Rotiz von ifren fogenaunten leioen nimmt, gar nicht auf ibre Rlagen bort, gar nicht nuf ibre Thratuen fiebt, in ibre Senf: zer nicht ftognt und liberhaupt gar nicht fie bemertst.

Spott wirts im Orunbe nur felten auf fie; er bringt fie wol jum Sdyweigen, aber beilt 
Seift fit niăt. Eie betrachten fid oaben sod) nod) immer als ein (b)egenftand ber 2luf= merfiamfeit - uno wusten fie vorber felbfi nid)t, worúber fie eigentlid Elagen, uno wa= rum fie in fós onen frillen Thranen fanft aers fiefien wollten - fo gibt ibnen iener Spott falter fiibllofer Seeten, die feinen Simm uns fein (E) füb! Gaben fúr ein 5 gerz, wie bas ihrige, bie befte G̈elegenlyeit; Sie ber trad)ten fich nun alb verfaunt, alb georidit von barten Nenfiden, fehnen fid nun um Defito beiffer nach einer verfhrwifterten Seele; flieben mit Inoiguation jene bohnladselnben Menfden von Eifen unb Stabl, merfen fid Derfuipen Einfamfeit in bie traufidyen 2rme, um on ungefiort fich fatt feufzen uho fatt weinen zu finmen et caetera et caetera.

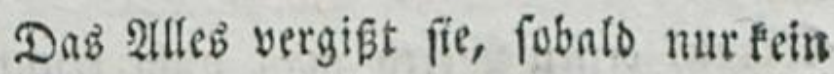
Menfid fie bemerft - tein Nenfich fie bes rádbelt nods bemitleibet, fobalo fie's lebhaft fublt, baj fie foblechteroings fein şefen auf Der ganzen weiten Erbe mit ifrem weinerlis dyen Brengeficht uno mit ihren barmbergis gen Senfzern interefïrt. Sie erreidgt igrent 


\section{IIf}

Broed nicht, uno fo wirft fie atid bas nichtomurige श्रittel freymillig von fich.

27.

Nimm Did) bes Iluterbricten, beb unc fidulbig Bevad)teten, Des :Berleumbeten," be verfannten Siedlicken, bes ungefannten oder niedergeorudten Seroienfeb an. Deffue ihm willig Deine İyur uno sein Szerz; Gỏre mits reibig feine Silagen und richte ifn liebreica auf mit beinem stoft. (siebrautb beinen

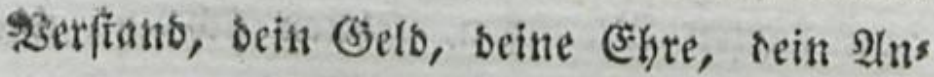
feln, beine gerbinoungen, beine Macbt sas ou, ibm Beredtigfeit zu veridyaffen uno peis nen 2 Berth ans Sidjt zu bringen. Drưfe ben Unterorictitcit, fráné ben unfchuldoig Seracbteten uno నerlenmbeten, erbittere ben Serfannten uno folage ben verdienfivollen Manu, beffen Berth man nicbt fennt oder nidyt fennen will, ourdis beine Şårte, burch

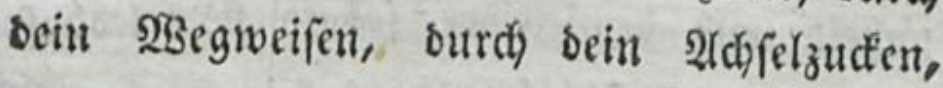
burch beine Ulntbatigfeit, ourdi beinen \&eid)ts finn, surch bein meniges S̃ntereffe fúr ihn, nidgt noch mebr nieber. Schame Dich nicht,

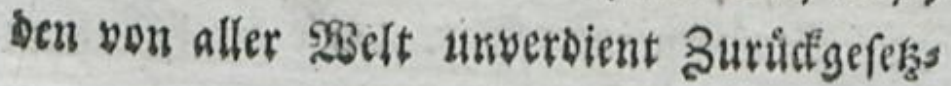




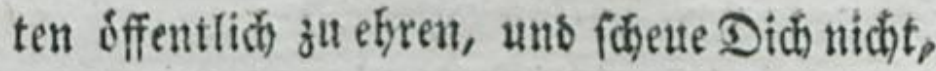
ben guten शamen bes siserleumbeten felbf

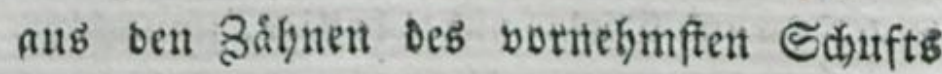

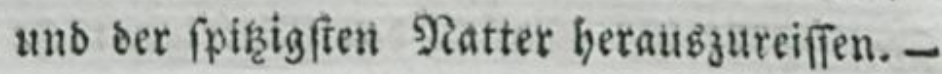
Id) - Du funtuft oft burch (Ein guteś Fort, ju ted)ter Beit gefproden, ourd) (Fine

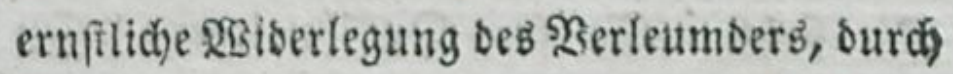
Fin warmes \&ob, burd) Fine (Fmpfehlung

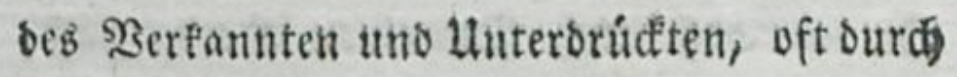

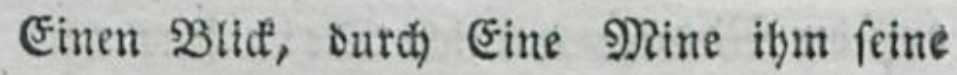

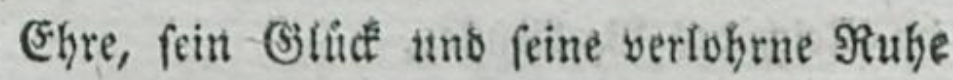
wicber geben. Du fannft oft burch einc Sleinigfeit, ourds eine Fúrbitte, ourd) eine Empfehlung, ourds eine Drobung, Durd (F:

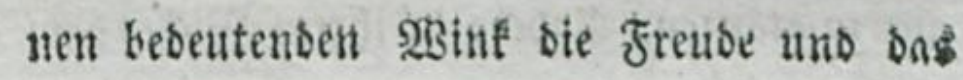
(5) lưf feiner ganzen Familie grủnoen. -

Bergif bas nie, wo Du eร verniagf́bene' Didh immer in bie Stelle bes Reident sen, fuble Dich immer in feine Empfinouns gen binein, uno bente' oft an baร 2Bort Эes fub: ,2:Sabrlid)! was ibr getban babt einemt unter biefen meinen geringften Brubern, basૈ

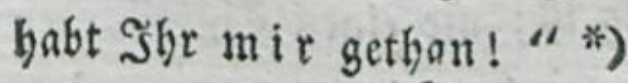

गृ. 2

28.

*) Natth. 25,40 . 
28.

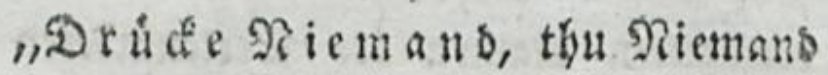
weh, wenu Dir's nicht beilige, unnad) thif

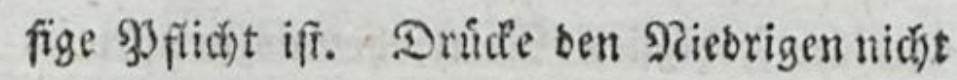
mit Deiner Mad. So wenig wie

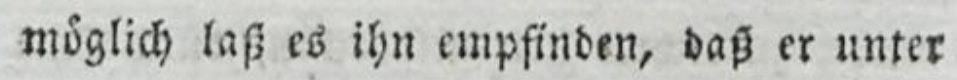
Dir fregt. Eo frey Du ifn irgend laffen burffi, Iás ifn. Dente, oafs er MRenín wie Du - onf aud) Du einen Szerrn bafr; Daf ber शiebrige vielleicht D e in feerr wirs im Reich oes :̧erbienftes.

Drưcfe গiemano mit De inem $\mathfrak{B}_{\text {er }}$ fano (5ib mur fo viel, alb Seber braudben fann, und jeige nie melyr. Gib nie blop

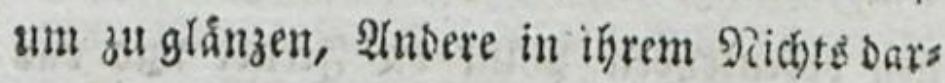
suftellen. Selebre, crleudate, madje mobt mit Deinem $\mathfrak{B e r}_{\mathrm{f}}$ tande, ober verbirg ifu in Dit.

Druide Fiemans mit Deinem 23 is, womit fo leidet uno fo bart ou sruden if. Rie erfaube Dir einen Einfall, ocr bem S/n, bern verbåditig madyt, wab ibm licb uns soerth uno beilig ift; nie einen Einfall, ore bem 2hoern web thun foll, ober nur wels

thitin 


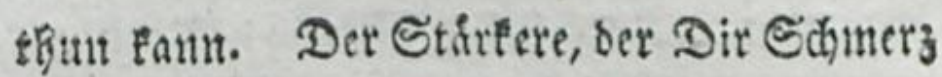
madjt mit feiner RórperÉraft, ift nidbt un menifblicher als Du.

Drutde aud Niemand mit De inem Ileberfu B. Beig' ibn nie auf eine 2trt,

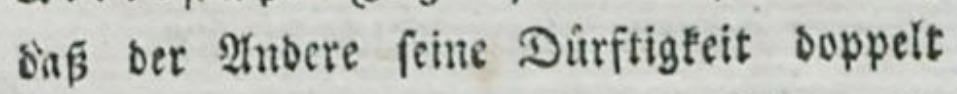
cmpfindet - Eine unerfannte $\mathcal{F}_{2}$ arte, dic (id) auds gute Renfchen crlauben, unb bic ooch wabrlid fein guteb $\mathcal{F}_{2}$ er yerrath.

İm meiften Gute Diđ Den zu orú f́en, ser am Leid)teften 3 oructen ift, ober Den Du sm Ieid)teften orticten Fannft. Sdjone der Sdwadh heit bes ভdjwas

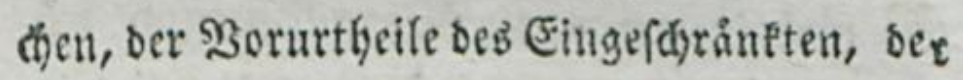

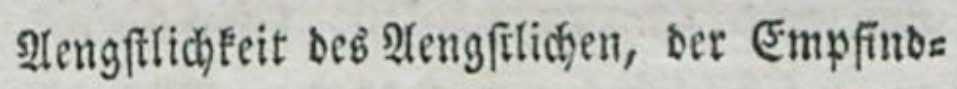

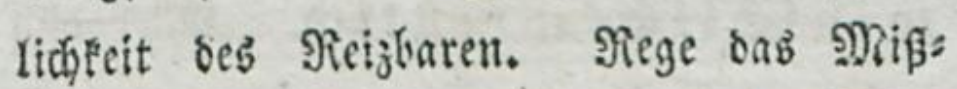

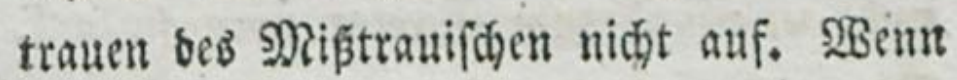
Du nur irgento abnen famnft, baß́ eir Senich georúdt, cinem Neniden wely ges madht werbe, fo balte zutudid, uno wenn

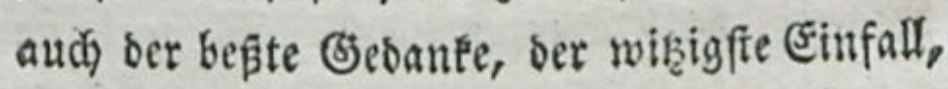
bas wabrfte $2 B o r t$ wáre - wenn Dir ane

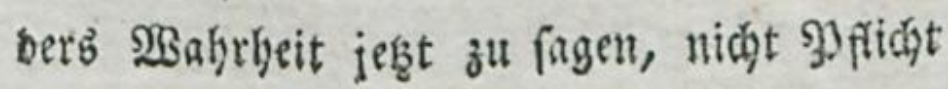
ift.

ก 3

attot 
II 8

Unt - D lieber Nenfd)! 2Benn gar Dein $\mathfrak{B}_{0}$ ort, Dein Befell, Dein Tadel etwas gilt; wenn etwa Thberer Sdjicts fat von Dir abbangt; wenn man nach $D e$ is nem (Seficht fiebt, auf Dein 280 rt borcht: sanu befonders nimm Did in 2 (c)t, bas Du nicht oriceffit. Ie gröfer Du bifr; ies mebr von Deiner 2) ter taunf Du weh thun bem Riebrigen, Der Dif bienen mus. *)

\section{9.}

Zebandele sie Unglúchlichen, die die Gdulo ifrer Thorbeiten, ibrev Derirrungen uns Dergebungen bi: fen und fobwer fuiblen, folsonend,

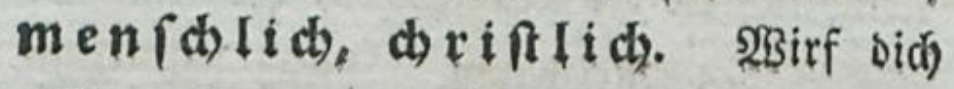
nicht unbefugt zum Richter liber fie auf. Sache Did) nidt breit gegen fie mit beiner Tugenb, bie Du vielleid)t auf ber Seite, wo sene fie beflectten, nothsurftig rein ex: bielteff, weil Temperament, N2angel an

నer,

*) S. Letralds Presigten viber raturtepte. 28 beft. bannover, 1790. 5. 55 . 
Derfút)rung, Bẹtegentheit, Utmftånbe ic. Dir güfrig, fo wie J̦enen ju gefägrlid waren, bie aber gewis auf mandyer andern Seite cben fo, wo nicht nod årger, bemafelt ift albื bic Tugent Desjenigen, gegen Den Du

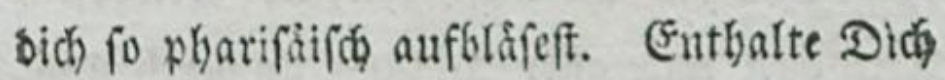
alles Moralifirens gegen fie, wem Du nid)t bejondern Seruf bazu baft; uno auch bann bebent, baf wiel Rlugheit, Menf(t)lidfect, Siebe uno yor allen Dingen aud Raá̃ uno Ştel baju gehoirt, wenn baв \$oralifiren in folden, wie in allen, Fallen belfen foll.

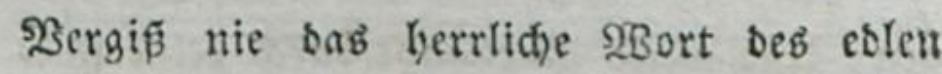
menfकliden paulus: "Eo ein Menfdy "etwa von einem Fefler úbereilt wúrbe, fo ,helft ifm wieber zuredot mit fanftmithi: "gem Beiff. Itno fieke auf Didf felbit, "Dan Du nidgt audb verfucht werbeft." -

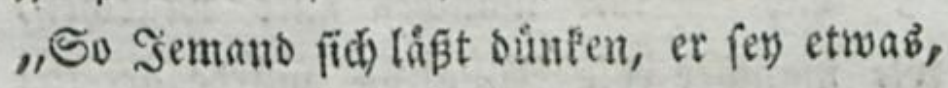
"fo er ood) शidetb iff, ber betruget fid felbft." "(Ein Seglider priffe fein Selbftwerf." *)

Szalte und behandele fie nicht gleidgats (b) fedte, vermorfene Nenfden, uns oride

$$
\mathrm{H}_{4} \text { Der }
$$

*) (5at. 6 , I. 3.4 . 


\section{20}

Den, ber outē bie Folgen feiner soerges bung obnebin fobon tief georidet ift, nidjt

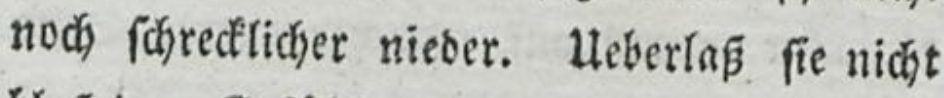
Gloź dem Gefühl der Schande, fondern fuche iknen, wenn fie nidgt wirflid) verworfene Senfben unb alfer $2(d)$ tung unwerth finb, Durd) 3 crveife beines SRitleios und beiner 2(d)tung wieder Butrauen zu fid felbfi eins z̆ffisen. Bib ilnen Mittel uns Bolegens beit, wie uno wo Du eb nur irgent, ver: magf, fich aแs ber Schanbe beraubzuarbei ten nub ifyre হugendent nus sid)t ju brins gen; Und wede iberbaupt burch beine fdyos nente, theilneismende menfoliche Befando. rung Muth uno Eifer in ibnen, einer foldgen Bebanolung burd) ifr túnftiges Setraget werth ju rerben.

2(ă) - wie mandjes gute Måbchen, wie mandber edle Jüungling wúrbe gerettet uno zu ben bejten Menfchen fich berauf gearbeitet baben - waircn fie nach einem unglidelichen Jebltritt, nach einer unbefons

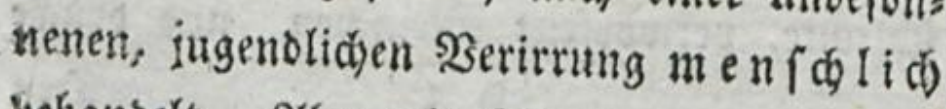
bebandelt. Sher mit Edyaubern murben fie 


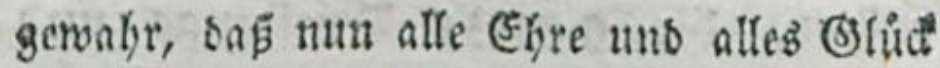
bes \&ebens auf immer für fie bahin fey, bap

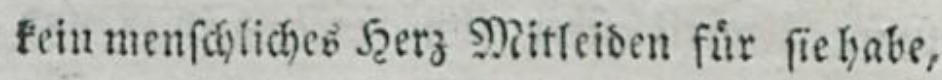
Ino feinc froffmung fúr fie librig blcibe, bie Sd)mad) je ju tilgen, je 2ldtung uno \&iebe wieber zu finton; man entrif ihnen alle 2(d)= tung wno nlles Butrauen zu fid) felbft, uno fo flolyen fie bann woll serjweifelung bem

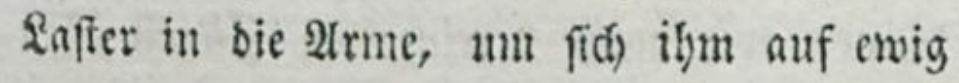
zu ergeben, uno ben ibm fich Sdyablob̆hal= tung ju fuden für bie ummenfd)lidbe llnges red)tigfeit, bie fie nidit verbienten uno gegen Die fie nirgents Shung fanden.

$$
30 .
$$

Etofi tiberbaupt nie Den mit barte suruce, dev fich irgeno einet verivung fajuloig gemadyt bat; ents balte oid 2lles EpIitterrid)tens, alles 2lburtiolno und 2lbfprectens uno al. Ies lieblofen Derbammens.

*) Das 5 an $3 \mathfrak{e}$, oer 3 an 3 echas

$$
\mathrm{H}_{5} \text { taks }
$$

*) Diefe folgende Stolle ift aub einem meiner 2 riefe genommen, bie icb im Iabibudae babe aboruden Inffen. 
ratter, die ganze Dentungs = uns

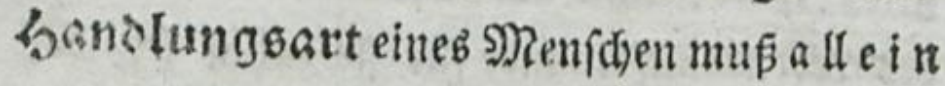
unfer Urtheif luber ifn beftimmen; uno o a rnach fann audb nur allein fein 2Berth ober Uluwertly entfbieben werben. Einzelne Rineamente obne গiudficht aufs (banje, machen sin menfd)lidbes (s)efidyt wes ber fdion nodh báplich, uno eben fo menig fonmen wir nach vereinzelten ăgen über irgene eine moralifbe Sobyfiognomie urtheis len. SBollen wir bas Leben jebes Men. folen zerftúd"eln uno zerfersen, unb baun nach Gutbünfen aนs einzefnen Fliffen mieder ein

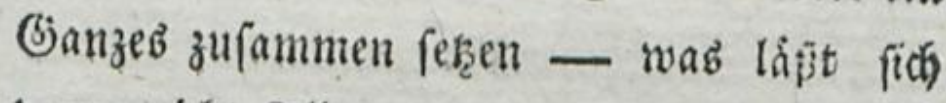
baun nidbt 2llles aus uns madjen! Dann fónnen wir nidjt felten ben sbifemidgt zum feiligen erkeben, unb ben edren Manu zum nichtbmurbigen $\$$ Buben erniebrigen.

Un's allen, obne Iubinabme, Flebt die Menfdibeit an, uno Seber, auch ber Sefste von unb, hat feine fobrocben unb fobimpf=

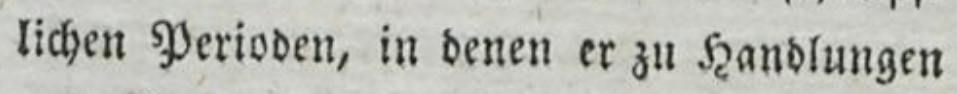
Ino Serirrungen fortgeriffen wiro, bie er sie redtfertigen fann und will - Seil Dem, 
Der folder goriobent in feinem Seben am roenigften jablt, uno am fonclliter berr úber fie wito - Er ifit ber E:s

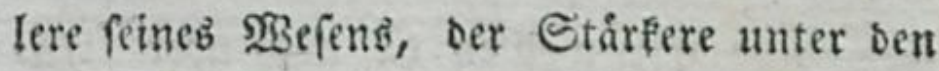
Ed)waden, Der 2tuserwálylte Der Zugens unto Der Siegercrone am nåd)fen?

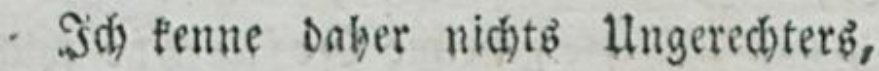

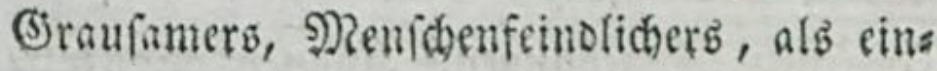
zelne Sdwad)en cinzelne \$erirrungen aub bem feben eines Nenfdien, ber gut zu fery frrebt, wenn er's gleid) nicht immer ift, aufzugreifen, unb fie z̆m aubzeidunenden Stempel feines (Sbarafters zu machen, um ifln bamit vor aller $28 e l t$ ju brandmareen,

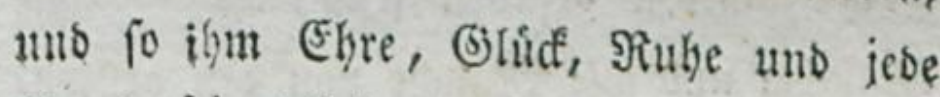
Frente feines febens mit faltem Şerzen zूम morben.

Sigie wenig und wie felten find wir boc riberbaupt im Etande, áber einzelne frembe Şandlungen ridgtig za urtheilen! Segreifen wir's pud oft faum, wie eई móglich war, baś pirfelbft uns biefer ober jener Hebereilung, \$ergeffentheit uns rer Selbft, Leidtfinnigteit, \$̧eritrung bes

Sieje 


\section{4}

- Geiftes odet Secrzento 2e. fóbuldig macjek unb Şandfungen begeken fonnten, sie allen unfern Birmofathen fo ourdjaus entgegen finb, uno wir wollten oa nod ůber 9 !ns bere riditen unb aburtbeln uno verbammen?

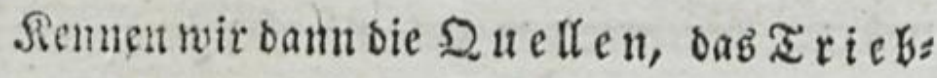
wert unto sie gebeime (befdi d) te foldher cinzelnen Sergehungen? Sơnnen wir'ş wif= fen, ob ser Biefallene der Berfifirer voet

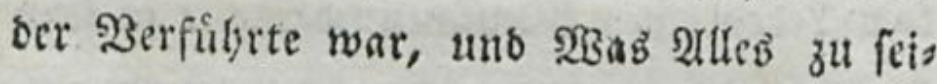
nem fall wirfte? Ilno find wir's fo gewifi, onf wir in glei doer lage, unter gle i d e en Ulmfrånden, mit gleichem Scerzen zc. nicht wirben gefallen feyn? $s$ - wie Mandier uno wie Mandje yon ben geftren gen Serren uno Damen ware wol noch tiefer gefunfen! Şeser bete bod) táglid babี: fůlve tuns nicht in verfuchung mit wabrer ferzengandadyt uno mit tiefenr (O) fübr reiner Selbft! -

Bebdd)ten wir's sodh: wie oft Rage,

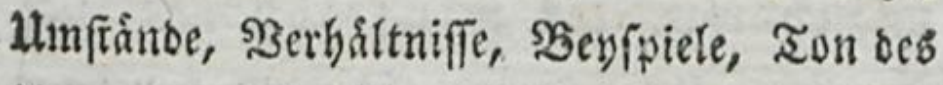
llingangs, Sectitre, lod"ente Bielegenbeit, Beifteşbetabung, f̧erzenşverftimmung, ges reizs 
reizte Reibenforaft, forglofe Hubcfangentycit, Uubefanntfdaft mit ber (befabr, bey ber wir oft ben erften Eđritt thun, ohne จaß wir's wiffen uns wollen, uns sem ban ber jleyte,

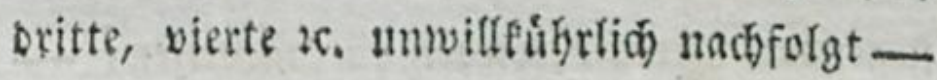
furz! bedaditen wir's, onf oft eine tau= fendfårtige, faft unwiberfeglidge Eoncurrenz von innern uns átfern Reijen, Antrieben anb Berfibrungen fo mandien guten - fo mandien eblen Nenfकen allmád)tig Ju Ner= irrungen fortreifen, die er von jeber ver: abfö)tuete, bie ibu benm profilichen Erma: den in ber Tiefe mit Sdrecfin mo Ent: ferzen erfutlen, unb bie nadjer oft feit

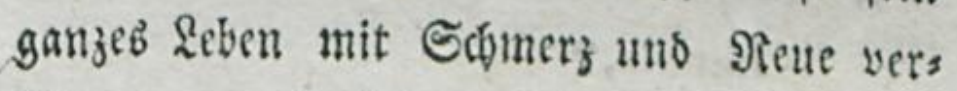
bittern; - beosidsten wir's: wie oft Mans der lange zeit mit månnlidsem Soutb uno mit månnlidger Staft jedem imern uns

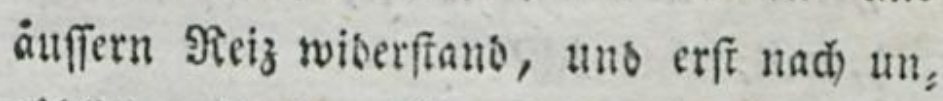
Jáblichen barten Simpfen uno múb/am crs rungenen Siegen (bie शiemano bemertt und शiemand in 2(nfolag bringt) ouleģt benno(n)

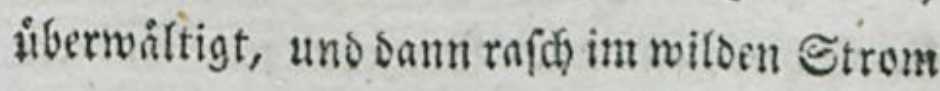
Def Reibenfdaft immer weiter uno findfer 


\section{6}

tho unauffaltbarer fortgetrieben wurbe, of: ne gleich Sraft uno פath uno Befonmens beit zu baben, gegen bie ftirmende Fluth anzufchwimmen uns fid aแt ferne Ufer mit fiegender Bewalt wieser binjuarbeiten; -

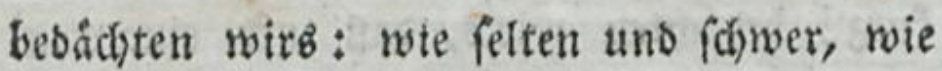
faft unmáglich auch bey Den bef́ten (Soruno: fäzen, bey dem reblidffen sistlen, bey beut talteften Temperament und bey bem gefers tefien Mana eine unter a If en Umfínden unerfatutterlitbe $S$ e ft $i g$ t'e it des Cbas ratters iff, uno wie oft aud ber befite )(en(d) borajens

"Ducimur ut nervis alienis tnobile liznutn, "

ober bie Rlage ङinebs ber ebelften Menfchen: "Das, Gute, das idh will, thu' id' "nid)t, uno $\partial a s 23 \delta$ fe, oas ta nicht "will, thu' ich"

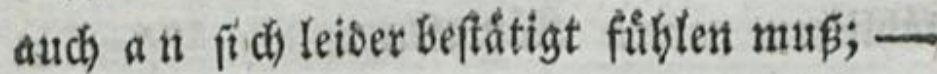
beoádbten wir's enslich: wie áufferft fd)wer eళ auffersem jebent Menf(d)en roiro, ůber

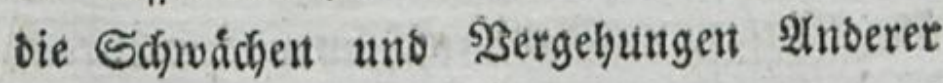
yollig unbefangen zu urtgeilen; wie

reid̆t 
Ificht unfre Eisenliebe (bie fid) fo gern vers foblen und obne bas wir'b fellft wiffen, vom fremben lintraut nábrt) oder ungün fitge Sorurtheile gegen ben Feblenben, গुar thenlidereit gegen feine feinbe voer gegen Die, die un feine Fegler erjăblen, die viels

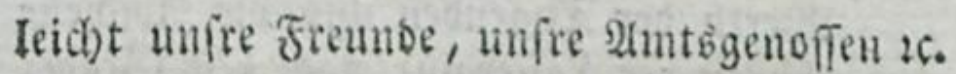
firto, oder gegen Die wir igreb Jangeb, ib=

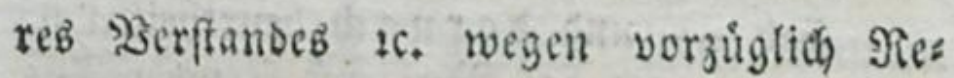
fpect baben - Eoflifion unfers Sntereffe nit bem feinigen, menn fie aud) nur funfs tig miglich fegn fómute - Berf̧̧icbenheit feiner Denfungsart, feiner (S)runbfăbe zc. von ben unfrigen - natưrliche פif̧billi: gung ber That, bie, losgeriffent aub aller Serbinoung, obne Rtifficht auf bie Hm: franbe, Huter benen fie gefobh, ober auf Den, ber fie beging, utb oft nod) weit frafbarer zu fenn fdeint, alb fie ift Betradtung ber Folgen, bie fie fúr unb ober Anbere bat, ober baben fónn= te - Felbftgenuigfames Befubl, wie wes uig wir einer folchen serirrung fábig fins 


\section{8}

find *) Furg! wie reicht fich tallieno Dinge ini unfer Uttheit mifchen, sie feine Febler vergróperm, feim (S)utes yerbunfeln und uns den ganjen Mienfisen ganj anders zeis

*) Die meifren Mrenfohen clafifificiten ben SBertly ber Tugenden und oic 2tbfheu= fid)ecit ber Saffer mur had bem (state, inbem fie berfertocn fáligig voce unfäblig fino - mo on r nach beurtbeifen fie

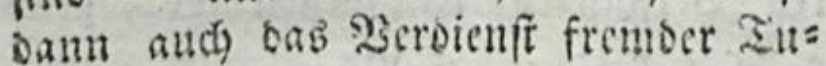
genten ober ben (3rab ber \$erounms lide Eeit frembar Bergefungen, uno nad

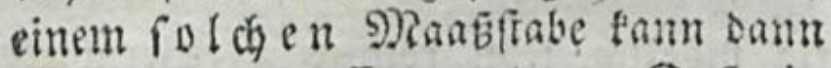
nie ribtig gemefien werben. Dás ein शRemich oas Seinige jull Rathe hált uno nicht jedem 2 ettler jumwirft fagt ber (seizige yon r.inem Eollegenverarge ich ihm liddst, aber bas er's bariun verfehen, Die Einbe auf feimen Şals getaben bat, bas ift abs feneulich - ift ihm gar nidst ju yers zeiben! - Eimmal mit einem blibs:

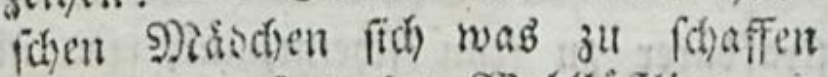

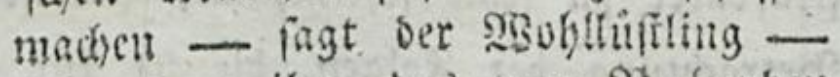
2Ber fanm ifym bab zum Strbrechent madjen? aber ids begreife nicht, wie

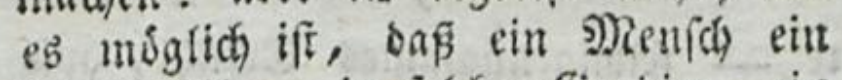
folder §ilz, eill jolder brobian, ein jolder et extera fenn taum, wie Der! u. F. w. So artbeilen uns berecintur Sie Mreiften! 
seigen, als ex if - bebåchten wir bug 2tles, unb griffe Jeoer flevisig in feinen

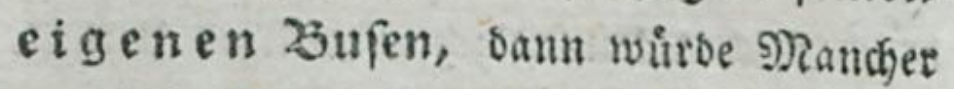
wot befutfanter, oulbenter, menfóliợer in feinen Hrtheilen lifer 2 ndere werben, iffen elyrlichen गamen feltener veridandfecten, eis nen Grettel haben an allen moralifden Tobte fohlägen, und drifflider ben Feblenden bes banben. - 2aber frenlid)! unfere beiligen

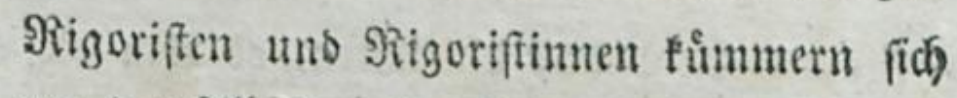

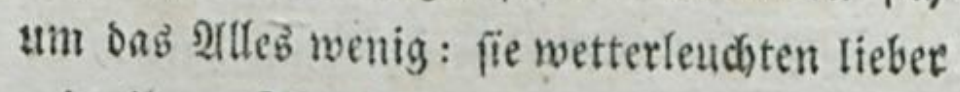
unit ihrer Theatertugeno, Gưllen fiá) mit

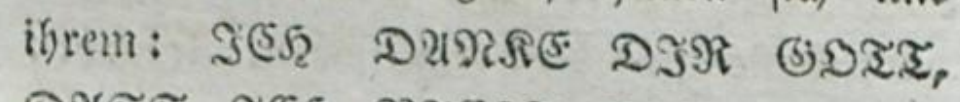

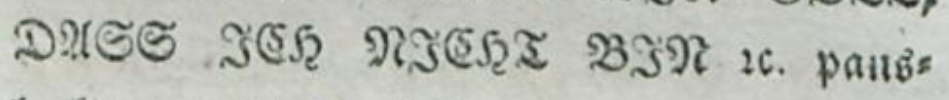
buctiy und felbftgenugfam in ibren গুy jarifáera Mantel, und foleubern Flud uns Serbers ben ouf alle fowwadbe, fünbhafte Erbenfins Der berab.

"Nictbts ift ungeredter - fagt ber eble (Ewalo *) uno शididt madt ungerechter,

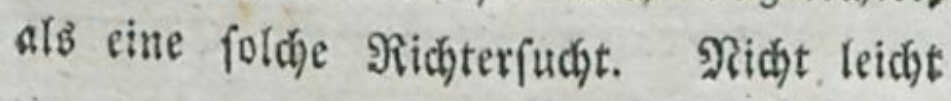

fann

*) ธ. Dresigten ůber Raturterte. 4 s. SReft. S. 35. u. $f_{+}$

ร 


\section{0}

taum man - gerabe von ben besiten, gros=

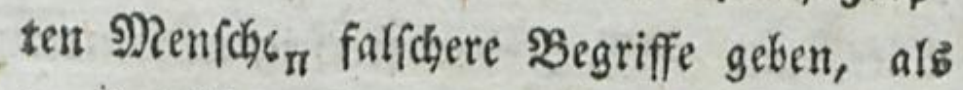
surch) foldes Esinfeitigez, voreiliges Utrtheil. 2Senn Semand fagte: da war ein Rosnig,

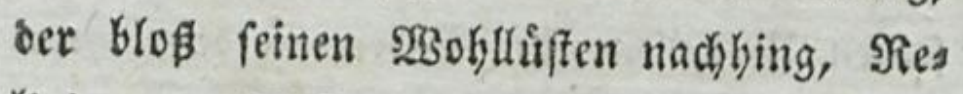
Yigion zu গRichts als politif(chen 2lbfichten

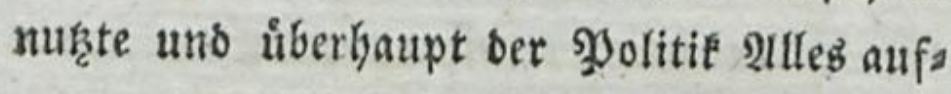
opferte. Itm Sinig zu werben, fegwur er feine Religion ab und wurbe patbolifo. (5x Yiefs einen Marfónall binrichten, ob ihm bie= fer gleidh fein Serbrechen befaunt batte. Eer jeugte adot Sinber mit feinen Raitreffer. - Ereylich fino Diefe Thatfachen alle rabar: aber wer erfennt in siefer Cinfeitigen $\mathfrak{b e s}$ forteibung Den feglervolfen aber esten bein=

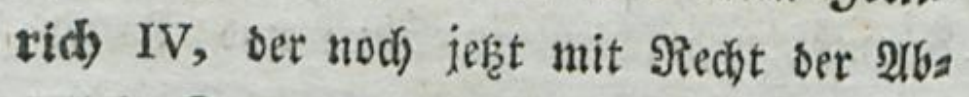
gott der ₹ranzofen ift? - $\mathfrak{B}$ enn man fagte:

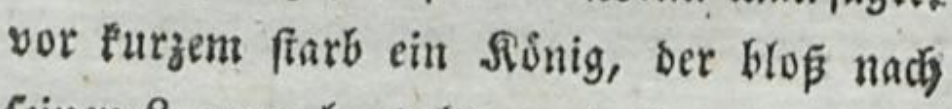
feinen Launen Gandelte, uns efer bie forreys enofte Uingerechtigfeit beging, er' er seffec ben wollte, oafi er geirrt habe. Sin liffiz

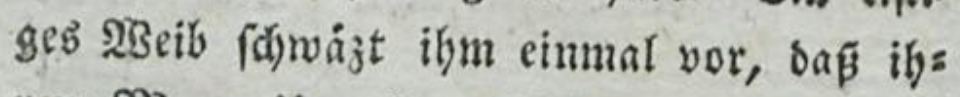
rem Mann Ulurecht gefcheben fey. Er birt

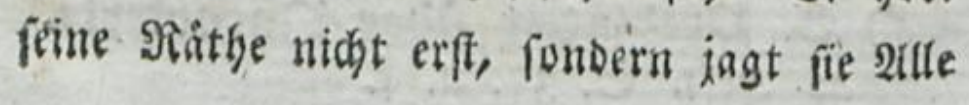
fort, 
fort, uno felbft feinen Grofíanzler, der von ber ganjen Sacje ?idits gefehen hat uns श्रidits gefeken haben fonnte. So if freys (ich) sie Erzåblung budffăblich wabr: aber wer erfennt in bem silde den, freylid) fes ften, felbft, wem man will, mand)mal aus Grundfâken eigenfinmigen, gered)ten, von fo viclen Seiten Eimaigen friebrits? - $\mathfrak{B e n t}$ Jemano fagte: ein gewiffer Sann, aแb bem viel $2 B$ efenb gemacht wird, mus felbft geftes ben, onf er geftoblen, fid eine Maitreffe ges balten, uno feime Sinder ins Jindelhaub ges forift babe: fo if aud $D$ a bieber wabr. 2lber ift biefe Erzablung alfein genug, uแn richtig über 2ioufieau zu urtheilen? - Itno wer erfennt noch ben guten, barmlofen, Doer wol gar eblen grofen Sam, went Du ein SBort, Fine Şandlung aub feinem Leben beraubhebf, obne zu fragen, warum er Dab 2 Bort fprac, bie ફ̧anblung vornabm; wenn Du Ein જुaar Febler neben einande ftellft, als bab' er fonft গiddts getban? Ilno oa fage Reiner: 2llles ift. Doch wabr, wab ich fage! - Dft ift's aน屯 Das nicht; ऽ2. $\mathrm{ft}$ 
oft if bie בhatfacbe verfellt ober ábertries ben! Sober we hn n's aud wafk iff, fo fint's barum bie úbereiften Edgluffe nicht, bie Du

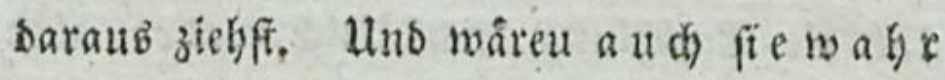
- wafrrició! Du bättefe nióst fo viel Jreus be, bayon zut reben, es losite nidjt fo fidjto bar Dein Rieblingegefprách, wenn Du $n$ ur SBablyeit fagen uno 3euge oer 2 Babrbeit feyn wollteff.

Schredtlid ift ber Edabe, ber-oft burch Dab Sichten gefiftet wirb, unb Sieiner iff Einen Iag fitger, of er nicht butch Fitt Raffermort cinem Nenffhen gefchadet babe. IBte oft wiro ber gute siuf eines barmlos fen Màbchens in foldyer Lâfergefellfchaft vers giftet und ihr ganzeb Błlưd zerffort! SBie pft roar ein Şügling feiner şerforgung nabe, uns sin abjprechentoes Hrtheil luber

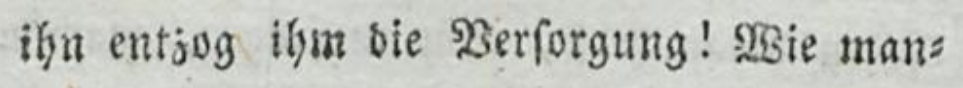

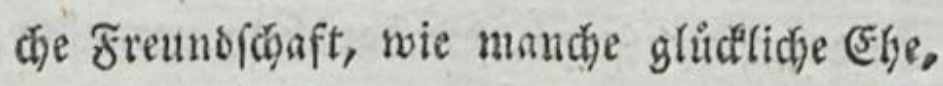
wie mandher Şausfriebe warb fonon ourch \&as

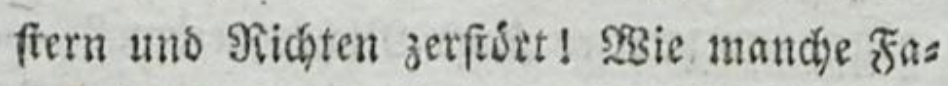
milie fâmachtet in Ulnglidé, weil úber ben Sgatbyater - vielleid) jum Beitvertreib - 
fo eit ridstenbes Şorr gepprodjen wart. Berabideuen follten wir uns felbfi, wenn nir fäbig fino, Nenfohenunglü zu unter graben aus Sangerweile. WBir verabfchenen ben Iytannen, ber mit Menfकenfreykeit uno Nienfidenleben fpielt; es if ben Ems pintofamen unfer Beit forrectich), wein sie

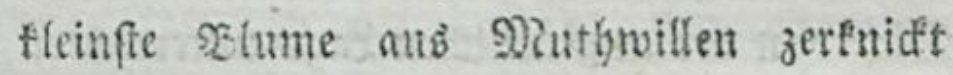
oder zerfluct wirb: aber wabrlid)! zehnmal

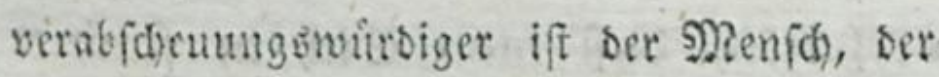

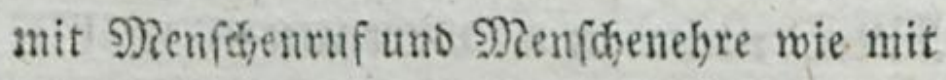
weften Shumen fpielt; ber bie Iugens, bas

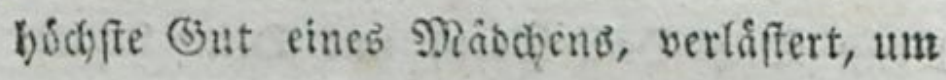
cime leere balbe Stunse zu füllen; ser einen Denfoten, eine Familie vielleid)t langfam

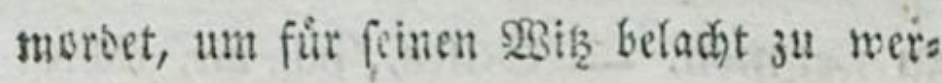
ben. D beß menfácnliebenben Beitalters, wo bies fo oft gefdieft!

\section{3 r.}

9) Rifde Did) nidt in Familienzwifte uns Galte Did̄) fo viel als miglich von allen Streis tigfeiten lunglucflidber Ebsegatten entfernt; geb' mit ibnen um, als riffeft und bemerteft Du gauz uno gar nidgtb von bem trauris

\$ 3 gen 


\section{4}

gen SBetbdttnif, in bem fie gegeneinandet felhen. Spabe nicht att, hordhe nirgents Gin, frage nicht, beutele nicht, winfe nicht,

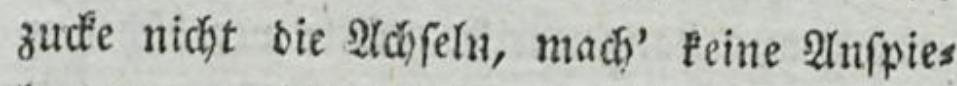

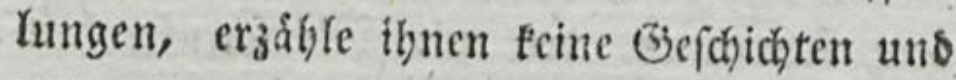

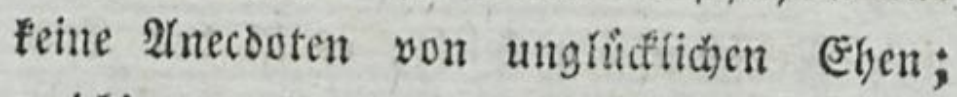
weich' auts, fo lange als miglid, wenn man Did) von ber unglưfflichen \&age unterrichten will - pucke es, fo viel Du famft, 3u verbuten, safí man Didb in foldben fallen nid)t 3um vertrauten roßble, unb - vor allen Dingen bite Did, wenn Didy Dein Beruf und Deine \$fficht nidyt allsoruaclich brzu zwingt - in fors d)en unangenebmen făllen $p$ art $t$ bie. 3u nelimen. - Eelten, faft nie, riçteff Du etwab aub, unb faft immer bift $D u$ ant Ende Der, ůber ben bende \$Jartheyen Gerfallen, um Didh mit Ulnwillen yon bans nen zu treiben.

$2(m$ besten banbelten anch immer fols che unglideliche Bjatten, wenn fie ifre traus rige Eage yor stllen, auth yor iffen treue: ffen Frreunoen verbeimlidsten, uno igr \&eiben mit 


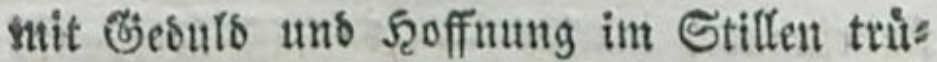
gen. Der Funfe elyelider. Dis̆barmonie leis bet felten, fehr felten \&uft, und wiro er uns vorfichtig verwalyrlofet, fo follagt er balb

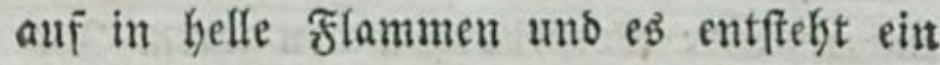

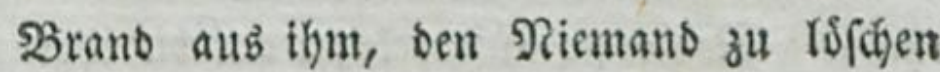
vermag.

Iber oft ift freylid bas \&eiben bes bes Dautrenbertgen (jatten ju grop und nieber=

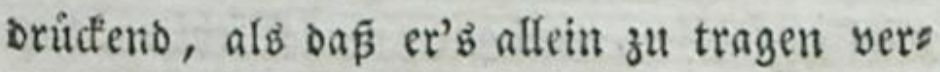
mag - unb graufam wåre es ba, ihm Theils nebmung unb tro̊ftenbeb Nitleib zu verfagen, wenn er's von uns forbern unb erwarten famr. - $\mathfrak{L a f}_{\text {a }}$ ihn bann feine filagen yoe Dir altifhitten; bebaure, rathe unb troffe ilyn, wie Du ifgn nady feiner $\mathfrak{l a g g e}$ nach feio net Lumftanben, nad feinem syerhalten 2 . rathen uno trofien famft uns barff; aber banoele vorfidtig wno mit weifer lles

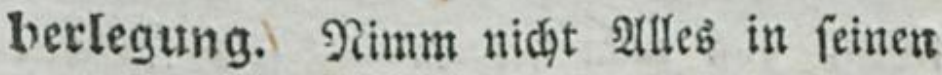
Slagen für treine ungemifhte $\mathfrak{B a b h t h e i t ~ a u f , ~}$ wie Dir's vorgeffellt wirb; felbft baร be巨́te 2Bseib und ber befte Manm, Libertteiben faft immer, wenn fie leiben, ifre şorwurfe, bie

$$
\text { \$ } 4 \text { fie }
$$




\section{6}

fie einanber matien - bas if liberbaupe bie SEeife jebes Seidenden. *) Szute Didd,

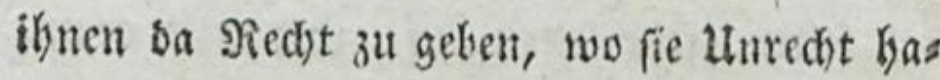
bent ; iberzenge fie viefmegr surch fanfte aber einfeucbtenbe \$orffellungen son ibrem Srr. thum. Mache fie aufmerffam auf bie श̧ers anlaffungen uno Sielegenheiten, bie fie viels leid) zur Disharmonie gegeben baben; zeig' esihnen ats igrem 3erbalten, dap fie felb ft wirflich felfiten, wol gar zue rff feflten, Daf fie fich bey bem Fehlen bes Batten meniga ftens nidjt fo benahmen, als eb filugheit uno Rebe ifgnen geboten. Sebr - pebr fels ten bat mur Ein Theil geedylt, fegr felten licgt bie ganje తdjuld nur auf Einer Seite; unb wenn ber Flagenbe autb) in ber Şaupt= fache Recht bat, fo hat er's ood gemeiniga lic) in to viefen Nebenbingen mis auf fo

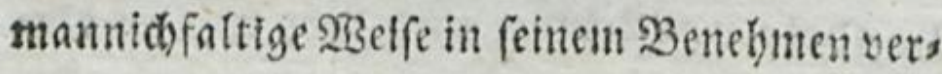
Reben, Dä́ man Jeinen ganz frey fprecten und Seinen ganz verbammen faun. Utebers fieflet

*) Animo dolenti nihil oportet credere, etiam innocentes cogit mentiri dolor. seneca in prov. 
frelgt bieb ber Troffret unb Rathgeber, will er ben antlagenden Theil ganz frey (preden, woer ifu fo entfdurbigch, als fonme fein 2erfefen gat nid)t in פechnung, - will er ihn mur bebatern uno troffen, unb ifh in feinet Selfitrechtfertigung beffâtech : fo mad)t er ben leibenten Batten, ober bab leibenbe

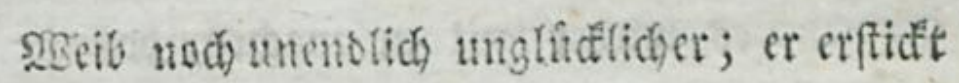
Daburch soltend jebes Finflben Riebe, wab nod) gegen ben 2(nbern tief in frerzen vers

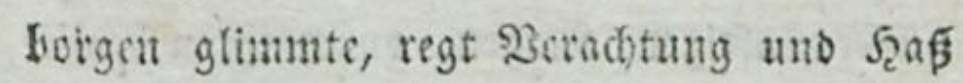
Dagegen in feiner ganjen Stirfe auf, vetieis tet feid)t zu taufens unbefonnenen, beteibis genben, frînfenten, unfeligen f̧anblungen, trennt beybe oft auf immer von einander, uno' wiro fo zum farecfliçen Eheteufel. Beffarte pie batyer anf leine sseife in ifys ren $30 r u r t b e i l e n$, bie fie gegen ben 2(nbern baben. Heberjenge fie uach beiner Mens fdomfenntnif, Dap ber Anbere wentiger, viels

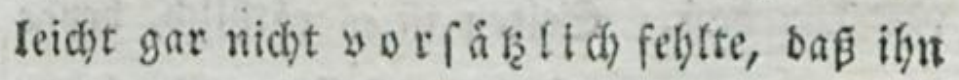
vielfeid)t ungrefutlidjes aupammentreffen ber Ulmfiảnoe, Berfimmmung, Eaune, Mistingen irgend eines જु\{and, Zemperament, Hebereis 35 fung 


\section{I38}

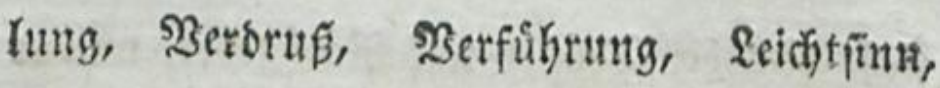
Ed)waiche 2c. 2c. zu feiner \$etirrung oder, su feinem firafbaren betragen fortrifs; daßs et viefleicht gar nidist baran bachte, fie baburch zu befcibigen ober ju franfen; ons er viets Yeicht feinen Fethler voer fein Shetragen fouon längit berettete, uno fajon im Shegriff gewes fen fey, es wieber gut zu machen, dáp fie aber butch ibre Empfindlichfeit, ourdi ibre Saunerey, burdy iffre Borwuirfe, ourd ifhre unvernuuftigen Silagen, ourdh ihren bewice renen Sangel an aller Edyounng unb an aller Delicateffe, Itlle wieder veroorben uno ihn oaburd zu bem 2etragen gereizt hastene bas er nachber gegen fie fortgefeşt babe. -

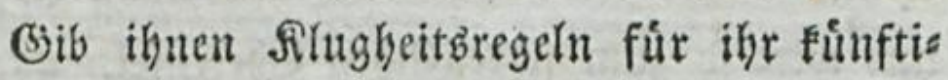
ges Setragen, fo wie \&age uno Umftánde Dir's rathen. Hebereile Didh in teiner Saa de; Ueberlege attes erfi wohl und nimm

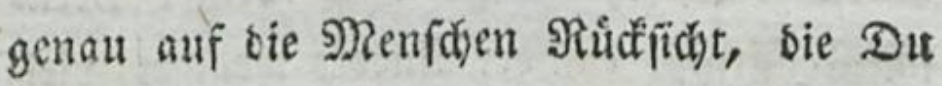
yor Dir haff. Selten if es ráthlitich, beybe Syartbeyen gegeneinder zuffellen tho gleidg= fam ङericht úber fie zu Galten; uno wer eb

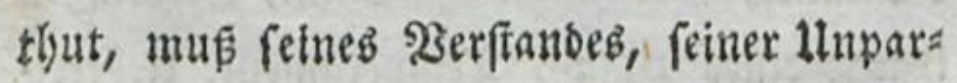
theis 


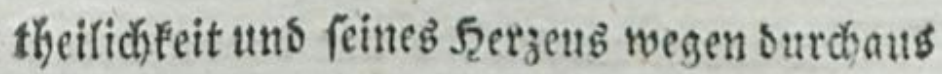
sie unbesingtefte slutoritât über Sende baben. Beffer ift es gemeiniglich, dap oer anbere

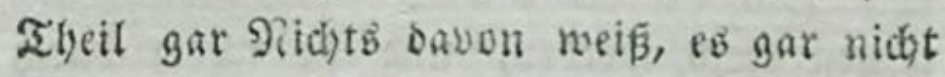
ahliet, man fey in oiefer Sache 23ertrauter uno Rathgeber: Ebelidbe S3mifigfeiten fins foft immer für Den, ber fie, verifoulbet ober unverfdyrloet, leibet, fo bemuthigeno, fo bers abferzeno, fdimpflich), uno es liegt - wes nigftens nad) meinem (5efübl - jo etwas unbef(hreiblich Nieoriges, Linwurbigeb, Entehs renoes sarimu, bas folde Necferenen uno Streitigfeiten I a $u t$, unb ourch frembe Nitwirfung gefdididtet werben - oaj ez ges wif felten ein Menf⿰亻 von einiger Delicas teffe extragen faun - er wiro fid baburd) felbfit verád)tfich, uno bies unerträglide Ges fubl rectunet er nun leidht dem (Siegeutbeil an, ser $\mathfrak{e b}$ veranlafite — mifd) menigftens feid)t sinige Tropfen Bitterfeit in feine veriofnte Qiebe, bie bann nadber bey Der fleinften \$Ber= anlaffung zu neuem Pigmuth oocr Unwils len aufgålyt uno bann weit arger aubfpru= belt alb vorber.

Dop: 
Doppert ungliceflich uns soppelt besaits rensiverth if inmer bab $23 \mathrm{e}$ ib, wem bie

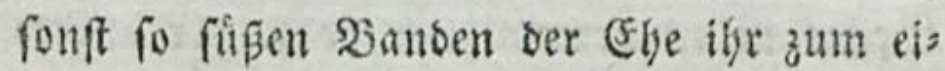
fernen Sod) werben, und fie weoer burch

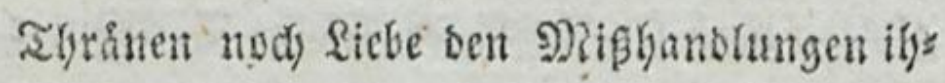
reb Du)rannen atsweichen woer feine \%ubs folweifungen bindern fann. $D$ - Güre ifre Silagen, füble igre Thrånen, babe Maite reib uno Erbarmen mit igr, unb verláp bie 2(rme niobt, went fie fonft finen frems, Feine Greunbinn bat, die ifre Serje fenn, auf bie fie fich melye vorlaffen fonnte uns mogte. Pathe ifre, trófte fie, hiff igr, fie,

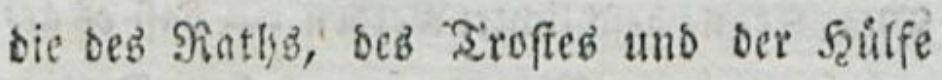
mekr, wie irgenb ein leibenber Menfd, bes borf. Etarte fie in ber Salfftmuth, in ber Giebutb, in ser froffnung und im Glauben an Gott. SBecle uno berebe in ifr bie lle:

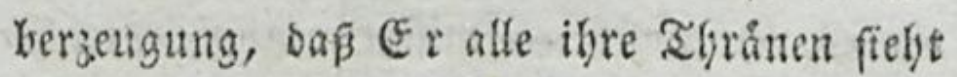

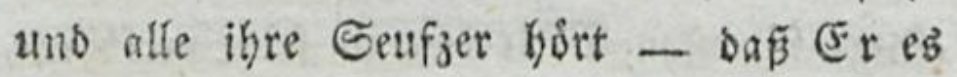
2tleb wei f, was fie leibet - bas er helfent fis nue unb helfen wolle, unb bafi bey

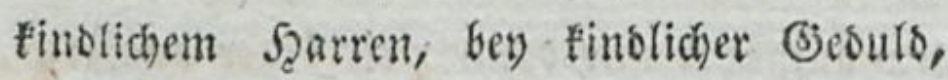
ber) finslicher Suverfict ganz gem i в aud fiir 
fât fie bic Stunbe ber Errbstung und Errets tung erfodeinen werbe, exfocinen muffe -

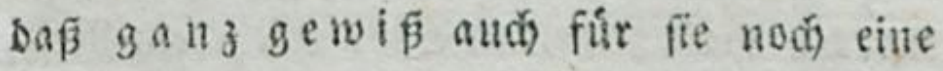
Beit tumme, wo fie Gott mit Frenbentgris nen preifen uno banken werse. Madhe fie aufmerpiam auf Das, mab Bott ief̧t butch ibe hartes Edjicffar mit ifyr will - fdárfe

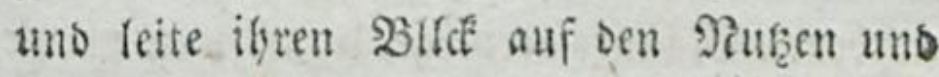

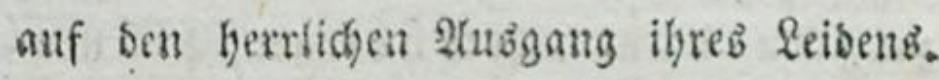

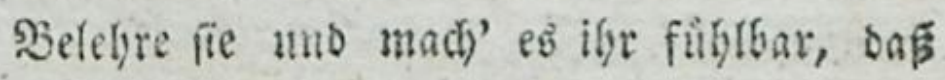
fie melge Siraft bat, iht \&eiben zu tragen, nls fie fid) fe!bfr zutrautet; bas bie weibfiçe Stárfe im Dutben ferbfi die mannuliche weit

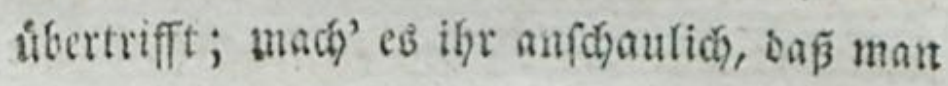
mit Neuth uno Feffigfetit im Setzen uno mit

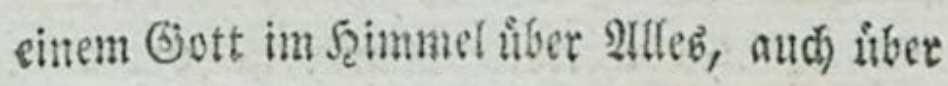
baв Cóntectlidifte, fiegen fanm.

Ermuntere fie und rathe ify, ferbfic ben unbandigfen Mann nod) mit fo yiel Sdjo: sung unb äufferer 2tchtung als móglid zu Gehandefn. SBarne fie, daf fie ihm nie zur Anzeit; voer gar in Gegenwart eines Drito ten Şorwúrfe madge, uno baß fie ifh nidgt fmmer mit Silagen unt నhrónen verfolge.

(3)i 


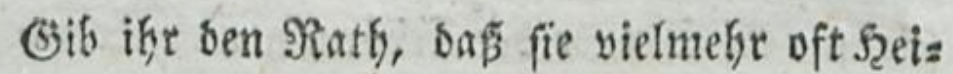
terfeit uus Sithe ertunftele, ibm oft ein freunos

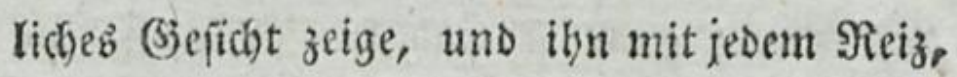
ben bie siebe ibr gibt, ifn an fich zu feffeln fuche. Sag' es ingr, dafi cin gutez, liebevola

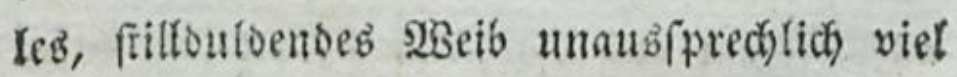

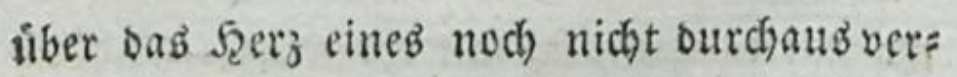
borbenen গ) 2anneb vermige, menn fie nur ničst

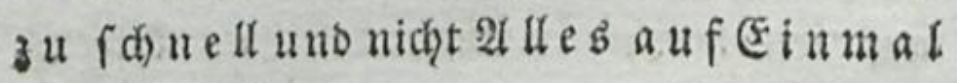
wirten wolle.

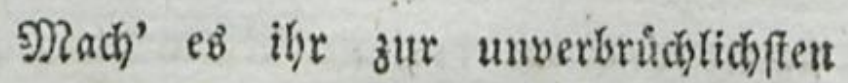
গুगficht, gegen jeden antern mit ifren Silas gen zu fdweigen - uber alle ifgre Seufzer uno über alle ibre STinen zu wacben, Damit fie nid)t bas (Befprich ber Stadt und ber Låfermåter werbe. SSBarne fie vor allen reofeligen, hiberoienfffertigen, leisigen, jis fchelnben Frau Gevatterinuen, Frau Sెa

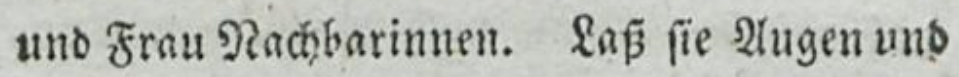
Shren vor ifnen verfdyliefen, wenn fie fid In ibre băubliçen Gobeimniffe einbringen uno bie qeiben ifgres frerzens ify abfhroigen uno abfliftern wollen; árgere, bobhaftere, giftigere, foleidjendere unb gefabrlihere Ebes

tell $=$ 
teufel faum ifyr felbfi Satan aus ber J̧\$lls

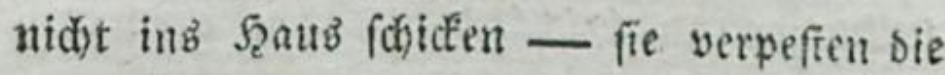
suft, in ber fie atbmen, unb baben fie mur Eimmal sjebor gefunben, bann ift an feit Entrimen mebr zu denten, - Sott feg Dem Mant uno ber fraul gnådig, in beren

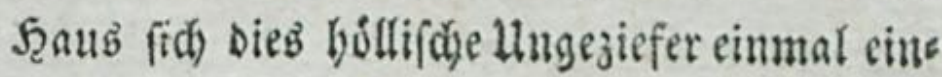
gentffet hat!

32.

3ebandele und priege den ti $x$ an Ien mit Iiebevoller Easmung uno unermioeter Gorgfalt.

feite bich, ihn, befonders wenn er feis ne Sirantbeit furd)tet, immer naळ feinem 3uftano zu fragen und ifn immer vou neus en weitläuftige $\mathfrak{E} e f(b)$ reibungen feiner Rrants beit madjen zu laffen; fie nuken zu शiid)ts uno fchaben oft unbefdreiblid): ") man macht ifu baburd) nur aufmerefam auf feinen leis Denben Suftant, man gewobnt ifn peine glidfe immer mur auf fich zu ridoten, peis

ne

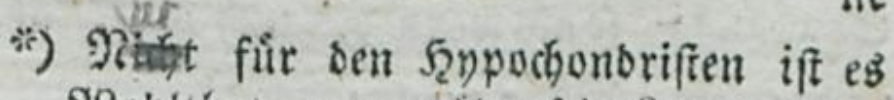
SBoblthat wenu er uber fein \&eioen plau= Dern tam, und if an mus man willig ein geouloiges Dhr leyben, weil ihm Das po viet Troff und Erfeidterung giot. 


\section{4}

ne Ceele immer nut mit traurigen 200 s frellungen zu befoliftigen, ben geringfen Umfino, die geringfe Soranderung in reis ner Sirantheit fir bida) fr bescutend ju bals ten uns er alsnet gar reidgt gewiffe csefabe in bem erwigen Fragen, uns wirs angfitid, mutblos tino verzagt.

Enthalte dich diberhaupt fo viel als mogs Iich) alles Fragens. Nichts quîlt uns árgert oft mebr, als bab etvige, unaufburliche, Inertråglidbe Jragen nad) Dingen, bie ein J゙es ser mit ein wenig Gefügl unb 2lufmerfams feit yon felbft miffen mup. Sen aufmetes fam nuf alle feine SBesurfmiffe, fucte ifm ro moglicy alle feime SBunfibe abutratben, uns bam frage nickt, fontern thu, was ou follf. grber melbe allen llngefum, alles Geråufch, alle Sibbeley, uno - vor allen grnbern dile 2lffectation uno alleb imponireno פ̧ers

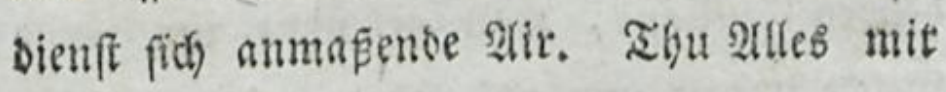
Seichtigfeit, ohne ben geringften Echein vou

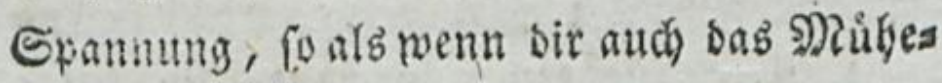
bollfie teine s)rube madjte.

Etmoiere im Stillen barauf, was uns mie igm भlles am woblfen madd, aber bam 
thu es mit einer Fatîrliø)ftit, als wents gar niđhtb 2lubers, alb bieb, und als menn's auf gar feine anbere SBeife getlyan werben foimte.

Semerff Du Hubequentidfeiten, mus etwa cin Siffen zurecht gelegt, sin Sুorbang an fonfer nicbergelaff n werbon 2 . fo ers warte nid)t erif feine Bitte, fondern biff ihuen auf der Stelle $a b$; aber fob:wake nidht erft viel oabey: ,Du liegf unbequem, Daв „bemeret aud) Niemano - id) will Dir bas „Niffen zured)t legen — Eo!! mu liegft „ou beffer!" "Die Eomme fobeint bir inb "(befidt, id will ben \$orbang nieserlaffen;" "die Jliegen incommobiren bich, ith will fie

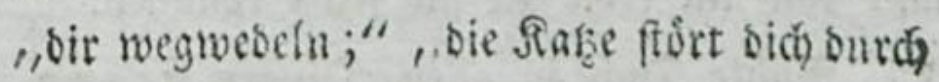

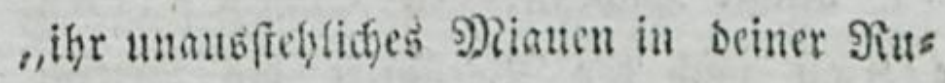
"the, ids will fie fortiagen 2c. 250 zit bab "id) will - id) will"? J్lif oem Llebel

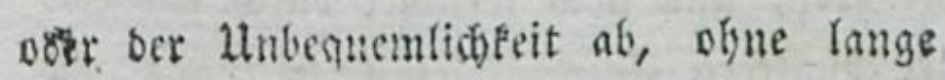
bariber zu fraben; unb vigne ifn ourch, oein

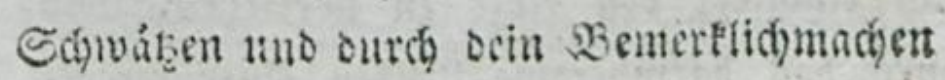
gleid)fam zum Dant nufzufordern.

\& shes 


\section{6}

Thut Nidits meft, als was gethan wers Sen mußs, was bem Rranlen mirllich anges nelym, ooer was für ifg wirflich wohlthås tig ift. Een nidit in ewiger Berwegung; zupfe nidst immer hier am Settuch, balt

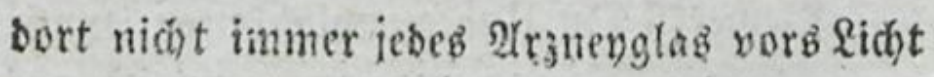
uno beriech nicht immer lebes \$utver; exa= minite vicht alle 2utgenblict feinen গুulb; Inuf niक)t alle funf Mrimuten zur Uhr, um zu fethen, ob es Beit zum (Einnelymen if ; frame unt fohleppe nidgt immer von einem Tifh zum anbern ober aus einem simmer ing anbere, uno tripple nicht immer uno ewig um fein fager ber. Die unnuflórliche

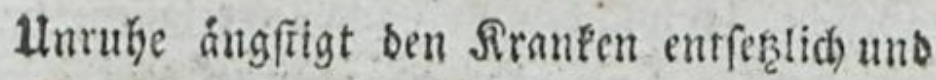
Idfift ifn nie recht zu fich) ferbft fommen.

Richte Dids in allen Dingen ftreng genau nach ber \$orfdrift bes 2(rzteB, uno láf Didh burch feime Sophifrereyen beg Siranfen, uno nod) weniger burd) bnz (s)e genfchnattern einer mebicinifden (3nnz, bie (iid) einbilbet ben 2 trzt zul úberfehen, noch burd) bas barmberzige $3 i t t e n$ einer unvera

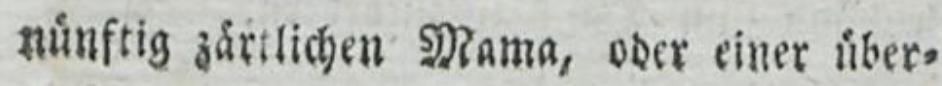

weidis: 


\section{7}

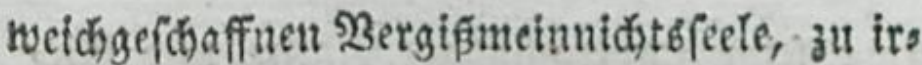
genb einer Abweidung verleiten. WBie fann ber $\mathfrak{A}_{\mathfrak{r}} \mathrm{t}$ einem Rranten linderung und (j) nefung verid)affen, wie fam er ridfige Beobe

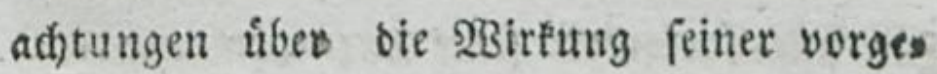
fáriebenen $\mathfrak{A r z}_{3}$ eyen madben, ben Gang ber Sranflbeit beuttbeilen und sarnady peine fers nere Bebanolung zwectuáfig einrid)ten, wenn

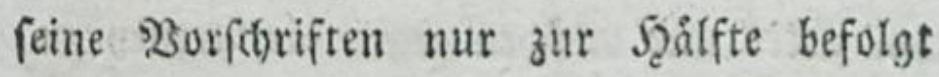
werden uno jeoer privilegirte oder unprivis Iegirte $\mathfrak{B a r t p u ß z e r ~ u n o ~ j e b e s ~ q u a d f ́ a l b e r n o e ~}$

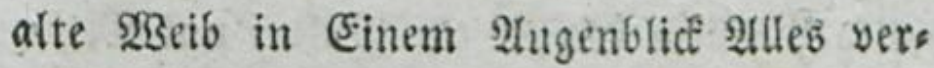
oirbt, woran er Tage uno 230 dénlang mit allem Sdarffinn uno mit aller Runft gears beitet hatte? Entwéber mus man bem $\mathfrak{2}_{3}$ t, bem der Sirnme fich einmal voll Zutranen bingibt, in Illem pit uctlid folgen, ober man muв auf allen glúdfldsen Erfolg feiner

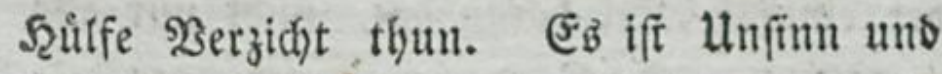
Tollgeit, einem red)tfd)affituen, weiren $2\left(r_{z} t\right.$ nur balb zu folgen, uno nebenbey nod jeder Sybille ins Maul ju bóren uno ibre abers wiß̨igen Einfalle für Drafel zu balten; felbf̣t bes gefabriofefte firanfe muß; wenn nidt \& 2 etrua 


\section{8}

etwa feine unvermifthare Natur mit Ries fenfraft bagegen auarbeitet - ohne Rets tung Daz ungliucliche Spfer bavon werben. Reich' iģm pinctlid) zur befinmten Zeit unaufgeforbert feine $\mathfrak{A} \mathfrak{r}_{j}$ neyen und ver= fúfe ibm ihren unangenthnen Gerd)mad burd) freundiche blide uno aufmunternbe

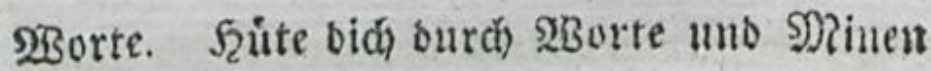

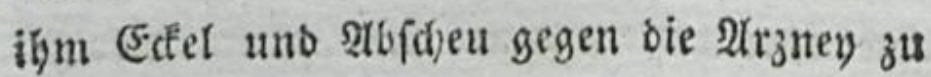
erregen voer, wentr er ifgn idjon empfinoct, iGn noch ju vermelyren; riffre ijm nidgt mit

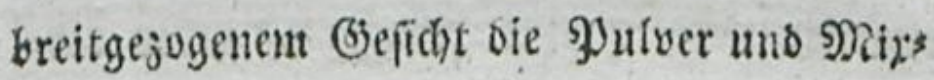
turen vor feiner $\mathfrak{N a f e}$ ein, uno bereite ifm teinen Trant', gegen ben er cinigen gBiber $^{-}$ willen fúblt, vor feinen 2tugen - ber Siran. Pe. mus folde $2 \mathfrak{r}_{j}$ menen nidjt eber fehen uns riechen, bis er fie rafd), obne langeb 3 befins nen, cinnehmen fann; fie verliert fủr Den, ber einmaf Edfel baran bat, nid)t nur alle. moblthátige Sraft, fonbern wirft aud oft graoe dab (segentlyeil, uno wiro ju tsstli, dem (jift.

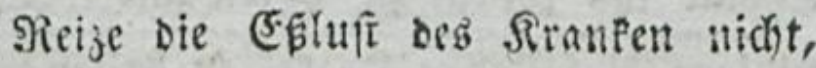
am wenigften zu Epeifon, bie ex nicht effen Darf 
barf, unto oring ify Nichts auf, was er burdaats nidgt effen mag. (5) fann in wol

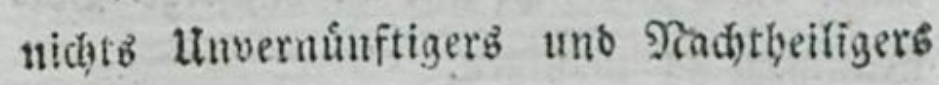
gedadt merben, als bab? Ino bod wiro fo mandie Rrantenpfiegerinn ourch diefe luns vermunft, laut Bengni aller 2lerjte, oft aus bartnifáigem Eigenium uno nod ofterer aus unmeifer liebe wein nicbt immer zur

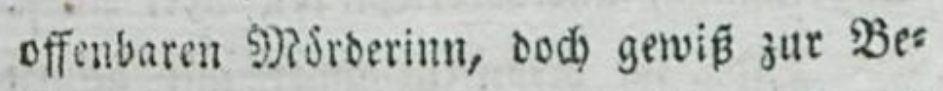
forderim langanlyaltender unbeilbarer firant: beitcu. Jd) feune eine Frau, Die ifgren tranten Mamu auf foldge SBeife mit einer niçt vom $\mathfrak{A r}_{3}$ t verorbneten Şaberfuppe ohne Erbarmen zu Tode årgerte. - Sit es nid̆t

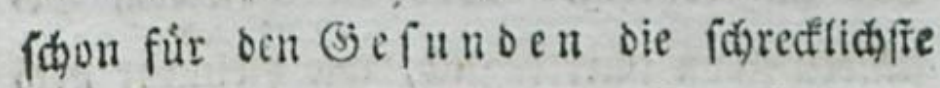
Duanl, mit fúrdterliden Complimenten uno unviserfieblidsen Heberrebungen jum Gemū von Speifen gejmungen zu werben,

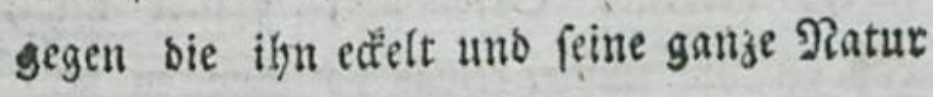
(iid) ftráubt? -

Feat Der Sranfe Neigung ober Begierbe etwas zu effen, ober zu trinten, yon bem ou nid)t meifit, of er ifgm oientich iff -

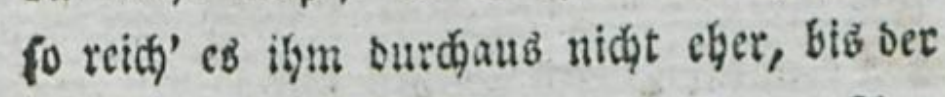

$$
\text { S } 3 \text { शrat }
$$


150

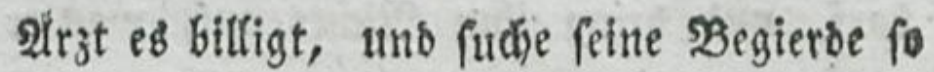
lange ourch liebreiche uno vernuuftige \$ors ftellungen zu mäßigen.

距 ben Rranfen nie zmeymal etroas fagen, nie zwermal um etwas bitten, शid)ts sweymal furbern - bie 2 Bieberbolung ber. felben Eactie foftet ibn oft unglaublid) vieb

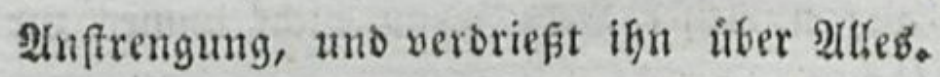

ฐapfe in Srantenzimmer mit Deinen

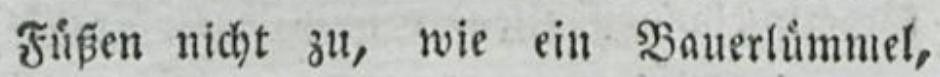
pchrene nicht, wie ein Marftidrener, fdarre niđt mit ben ๔tủblen, mirf uno poltere

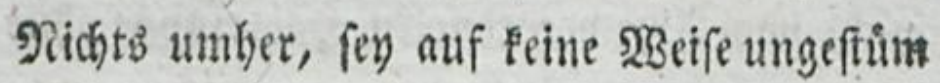
und ungebebroig. Das $\mathfrak{A}$ lles ift bem bies funben ficon unertriglich, wie vielmelsr nods bem Sranfen!

Martere unb langweile ifn nidjt surds fabes, albernez (sefd)wåţ; glaube nicht, oав ou ifm immer etwas vorf(h)maden mús

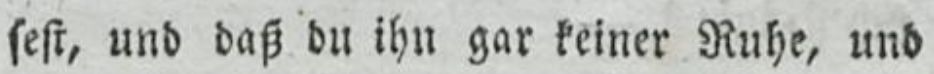
nie fich felbft uberlaffen birfeft. - §ube nuo Stille ift oft unausfpred)(ich wobltbatig

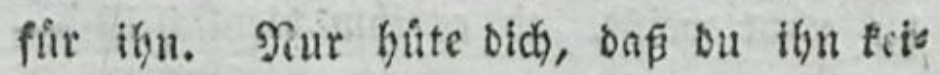
uen beunrubigenben Sorgen unb teinen Gerzs quณ์ 


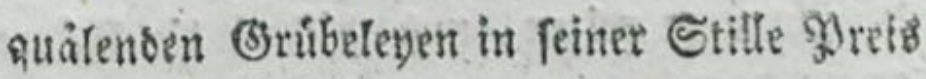
gibft.

Bermeibe alle Itnterhaltung, bie ibn auf irgento eine siseife angreift, bie beftige Qeidenfidsaften erregen voer feinen Ropf zum anlyaitenden gracboenten fpamen fonnte.

Ertrage feine Launen, feinen Sigenfinn, feine (brameley, fein Zanten und Edymálen mit friller Sanfımuth uno fdonenoer Siedulo; erbittere ifn nicht ourd 23ioerfprud); wolle nid) 2 (lle beffer wiffen, als Er; merbe nie mude im radugeben, nie bitter ley feinen Slagen, nie mifmuthig bey finem Iabel, nie auffatirent bey feiner j̧eftigfeit ; verfeß̨' bid immer in feine ange, uno benf' immer bar= an, Daß̄ bey einem leibenden ESrper autá. bie Seele lriber, und mit einent franfent Yuge aud) Dié Seele Shaudjes anbers peben mus, als ou mit beinem gefunden Stuge fies heft. Eigenfinn, Banffucht uno Rechtlyabe? rey. finb febr baufigge Febler Der Sranfen; - redine Daber foldse figegler nidit feinem J̧ers zen fonbern frinem Reiden an; vermeibe fo vial als moglidy alle (s)tegentgeit, peine $\mathrm{Si}$ शifis 
9) Riflane zu reizen, uno milore die Saure feines şefens burch fanfte Edyoumg uno liebcuolle Yjflege.

Entferne von feinem sortte nlfe gute Sath: gever, alle weife. Frnu Sonajen uns alle Suacfalbernoen 2 Beiber. Sic bringen ifu-

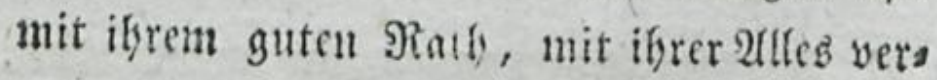

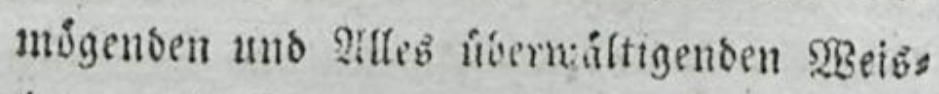
beit, mit ifyrem Siccemiren uno Meiftern Der 2orfariften bes 2 rrztes uno mit bem 2h= preifen uns $\mathscr{A}$ fuforingen ifrer Şaubmittel uns ifrer beiflofen Suadfulbereyen fidserlid

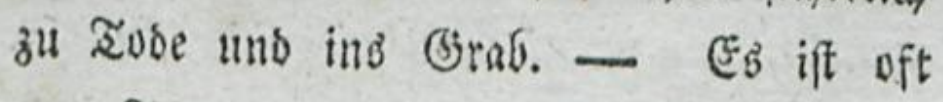
zum Tubtladisen oder zum Tobtírgern, wem

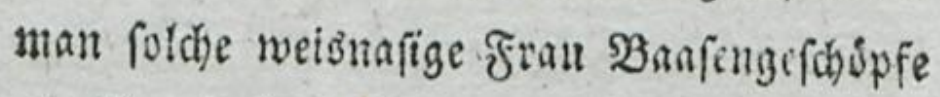
am Rranfenbett bort und fiebt. " शRun wie

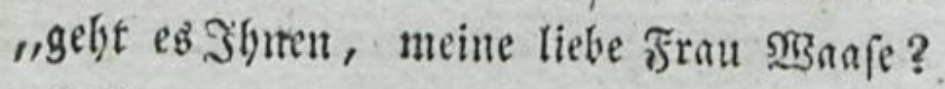
"IBill es nod) nidgt beffer mit Sie werben? "(5) bes bauert ia auch erichrecflich, merhort, „abfáculich 2c. lange! - $28 a b$ baben Sie "Denn fü'n Docter? - Den?!! Э wie "fommen Sie aud an ben? פR e i ne $n$ Docs "ter follten Eie nehmen, Der curirt \$̧nen "gewif in zwen, orey Dagen" - "গRee, Fran 
"Sraut Bovatterinn" - fällt sant sine 2 (nbere ein - "wenn id Eie rathen „,foll, fo Inffen Sie Docter Docter feyn!

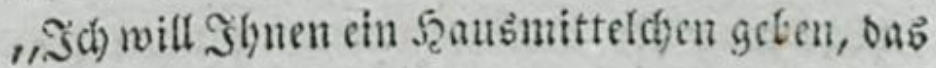
"Sic beffer uno gefáminter belfen foll als "alie Socterb mit allen ifyen Sु) "Siarrenpoffen; Damit bab id) f(b)un fo 9lans "clien gejund gemedit 2 . (uno mun folgt cin raifonirtes Stegifter aller ifyrer (Suren.) "2Bab̆ haben Sie denn da für Medicin?- frägt bie Erfte wieber - laffen Eie bod mal fes „hen! (Sie wirb herben geholt, befwmet, befduittelt, berodien, bef(hmedte, ) "S Ferr

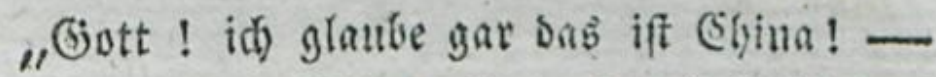

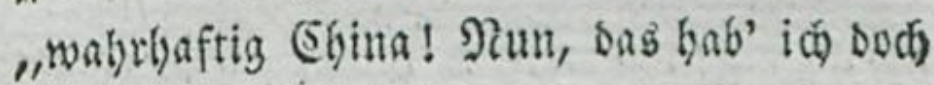

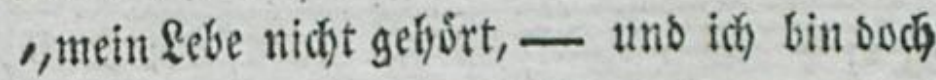
"furmabr nifgt von Gieftern ber - baß

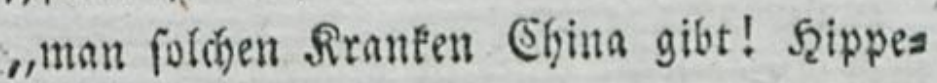
„fafana múffen Sie nelymen, bab ifi bab „eikigige গlittel für Sie, wemn Sie wieder "gefuno werden wollen zc. uns danu miffen "Sie ein gut SSlab rotly BBein trinfen" "I, Aber den Gat mir der Doctor verboten!"

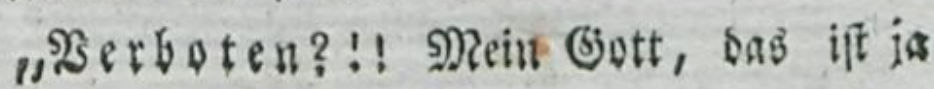
\& 5 1100B 


\section{I54}

„oab arterbefte, was Shnen bient - id "Gatte mal eben ben aufall, uno juft roth "SBzein war's, ber mich allein wieber half! "SBab mag ber Docter wol mit folden ein „fáltigen ßeroronungen wollen? Nein --

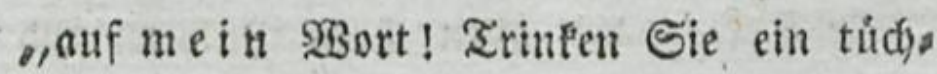
„tiges (jlą voll - fagen Sie bem Doctor „i ch) bătt' es gefagt" u. f. w. Daburch wirb mun oft felbft Der vernimftigfte Sirante to angftidi), fo sweifetns, fo mistrauifo gegen feinen tretten 2 trzt, fo muthlos in פefolgung feiner bellfainfen Borforiften, baf, wenn er auch wirltich alfe Eybillens rathfdalage verwirft, ber $2\left(r_{j} t\right.$ bod ummógs Iidg fo auf ifn wirfen fann, wie er ges than baben wurbe, wenn jenes Duadfăs ber = uno medicinifhe sgeisheitspad yon feia nem Bette wåre entfernt gethalten. *)

Ento

*) Nichtb if unerträglicher fúr ben $\mathfrak{A} \mathfrak{r}_{z} t$ fagt Ztmmetmonn - alb \&eute um die Franten zu baben, bie in ber $2 \mathfrak{A r}^{3}=$ nevfunft biz auf zween ober brey ber ges meinften sjegriffe ganz unwiffent fino uno biefen armerligen Edyatten von 23if: 
Entferme diberbaupt alle âberlaftigen Bes fudjer uno alle zubringliden 2 ijitemmacher

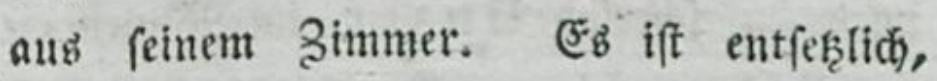

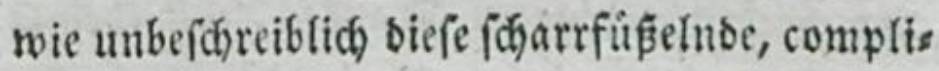
mentirende, fabe, edfellyafte, fonnadffüdtige gienfdienclaffe ben armen beonurenswerthen

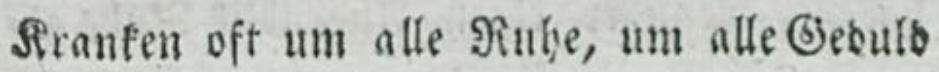
und um allen \$erftand bringt!

"Ëin

2Biffenfdaft für ben ganzen Umfang einer fo weitláuftigen uno fefte verwie= Felten Sunft balten, einer Sunft, int welder ber gróste unb geńbtefte (jeift am Enve der glorrcidgfen \&aufbahti feine Unwiffenbeit vóllig erfemnt, went er Dab, wab er weî́, mit Demje: nigen vergleid)t, wab er niđgt weî́ ; einer Siunf, von beren 2 tubs úbung mir einft ber Sianfert. erffe \&eib= arzt, Fren\% van Gwieten, to fimmreid als wabt geforieben hat, fie fer) Das ficherfie (Begengift Des Stolzes. Sn ber Elaffe biefer armfeligen \&eute baben alle ungefirten Xsseiber ben Rang.

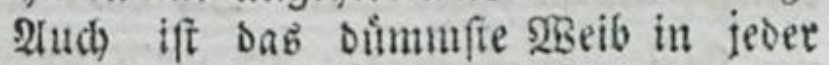
Stadt Dab fraupt ber Medicin von biefer Stadt.

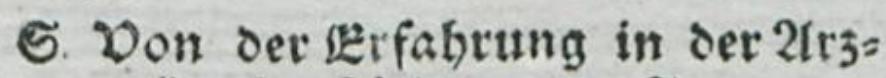
neptunft, Särid 1787 . S. 343 . 


\section{6}

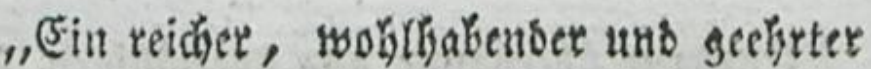
Nami, der mit ciner Sirantbeit befalten roirb - fdreibt Un $\mathfrak{z} \mathfrak{c}$ im Pamen cis nes folden geplagten Siranten, nit eben io

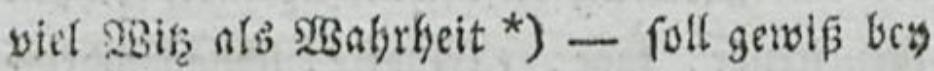
oer gegenwartigen \$erfaffung viel ju thun Gaben, wenn er einen 2lugenblie Rube ges niesent mill, um fich mit (5ott utmo mit fich fetbfit zu befpreden. Eie birten fid) nur ber bey) uแล fo fohr cingeriffenen Gerwohnteit ber Sirantenvefucise erimern, um ju errathen, twas iŏh eigentlich lagen will. Ein anfelgn=

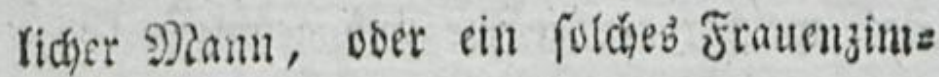
mer birffen $e^{b}$ nur eimmal befannt werben iaffen, bafis fie fith nicht wohlbefinden, fo

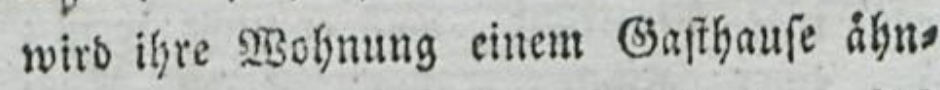

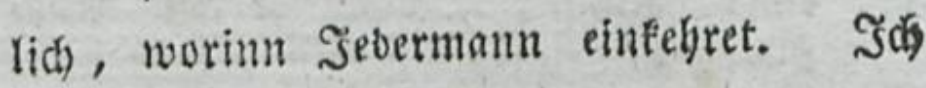
babe felbft Erfagrung gievon uno weis am beften, wab ich babcy ausgeftanben habe.

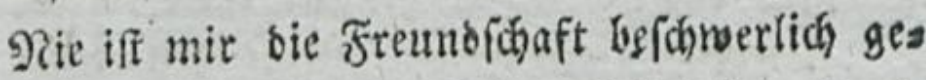
wejen, aib̧ zu biefer Zeit. Șd babe oft eis nen Streis yon Freunben um mein Siranfen bett herumgefoben, der aus zelg, funfzegn unt

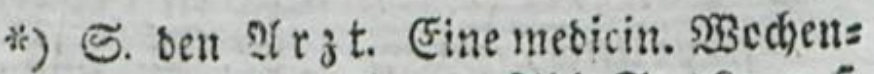
int. Fenmb I760, 2r. Thl. S, 380 II. F. 
unto jhanjig \$erfonen beftanben bat, uno wovon ein Şeber bie geringen Baben, welche er bejâ, anwenbete, um mirben 211 theil 3 befdreiben, ben et an meinem feiben nabme. Unterbeffen verniffelte fid) bie grope (5) efellfd)aft in weitläftige mir entweder febr gleidggultige, oder gar cefelhnfte Befpråche, rooran ids, weigen 2angel Der Taubbeit,

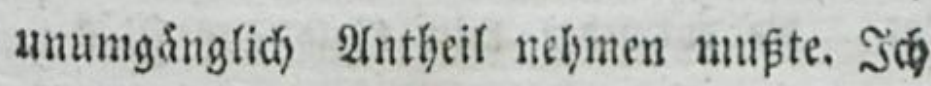
fann \$then unmogltch befdreiben, wie zers fireut und unubig mein Simutb wurbe, went ich bais eill netes Seyleid, balb ben $34=$ ftano unb ber Sndjen in Sreffen, in Echleficn, in গুगreแpen, am Rigeine, in Englano mo in Simeriea, bald bie SBegebengetten bec Stadt, balo die SBunfide eines Freundes, Der fich empfabl, balo die Jquffungen einç Pinbern, Der noch ?uft ju fig̨en batte, baid andre Dinge vernebmen muste, bie mid) frets anb einer rgelt in bie anbere ver: fetsen, ofne baß id in meinem Jemufe -tmo Dette mein eigner feerr uno Freuno wers ben fonnte. - - - Sagen Sie nur,

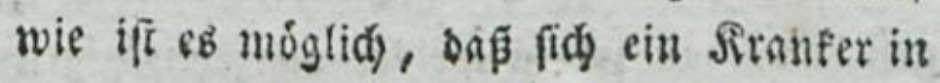
einex. 


\section{58}

einer foldhen Caffeefdente betubigen, unb fein (B)muith zu Dem erbeben fanu, ber ibn

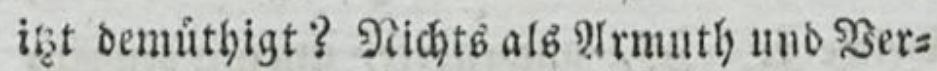
adstung fann einen franfen Renfden vor biefer (5hgre befduş̨en. Denn fo liebreid) fid die $23 e 1 t$ gegen Siranfe in ibren Befucben ermeifet, fo fiegt fie boch wol zu, baf fie ibre Begenwart nicht an \&euten verfd)wena Det, Die entweber fein Gielo auf Sinfen, oder feine Ebrenftellen zu belleiden, ober feinen Titel babèn, ber fie betleibet. Sach babe zll meinem Ungfúd ein Sapital von vierzigtaufeno shalern und einen Titel, ben

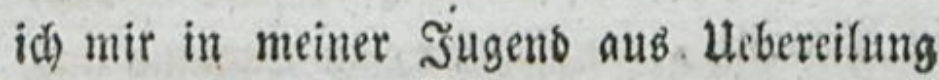

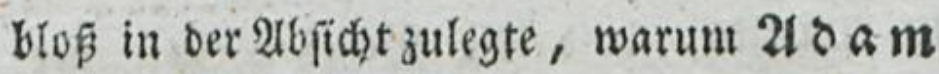
im Sarabiefe ein Feigenblatt yor fich nabm. Diefe unglúdtidge शarrbeit unb. oer efyrs wúroige SGarafter meiner vierzigtaufend Thas rer baben mir ein fordbes allgemeines Mits leib erworben, bas man rafenb werben wolls te, mich bald wieberbergeftellt zu febn, uns

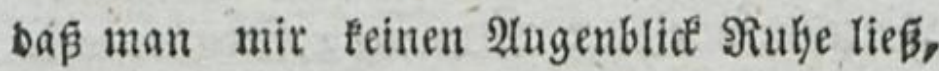

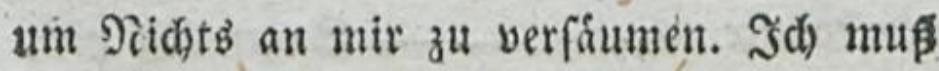
gefrebei, baß ich in meiner vierzebntágigen Siranflyeit in eben bem Buftanbe gewefen bin, 


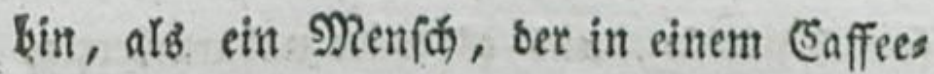
baufe bie zeitungen lefen uno beurtbeilen Gort. Sod babe weber an meinen eignen, nvd) an ben Suftand meines $\mathfrak{S}_{2} a$ fes benten fornen. Reine গুbantafieen waren nidgts 2nberz, als Die Giefpenfter ber aim Tage

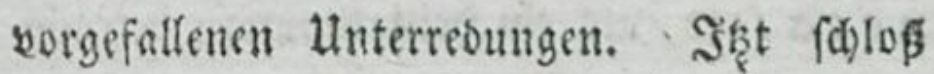
ich einen bonorablen Frieden fur Englano;

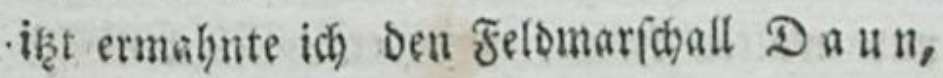
Dresben zu erhalten; i̧̨t nóthigte ich bie

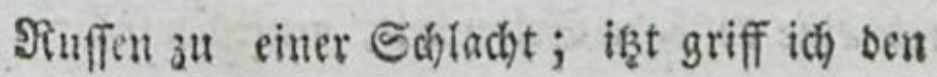
Rounig yon झreusen in feinem verfhanjten Eager an; id́) nabm Quebecf ein; erlegte ben Beneral $2 B$ olf; und half ben Englänern it Lonbon Sictoria rufen, ba unterbeffen oer भrrebiger, ber neben mir faf, erwartete,

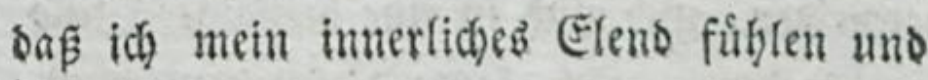

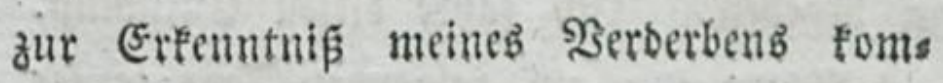

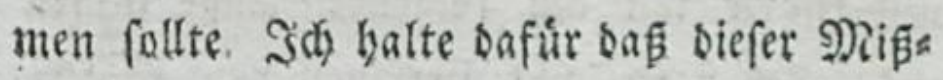
braud) mit ben Siranfentefucten, bie nidgt verlangt werben, ein wabreb 2 artyrero.

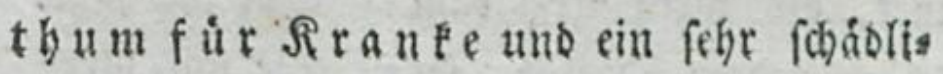
der Fehler in ber \&ebengart fen. (F⿱ ift waht, ein Sranter bat zumeilen Troft uno Zua

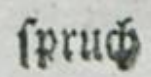




\section{6.}

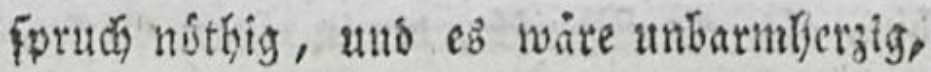
ifym benfetben zu verfagen. Atllein es if umfinig, auf biefe ङefabr grabezu zu gebu, ihm feinen Dienft auffubringen, Dber $\mathrm{C} b$ iiber zu nefmen, wenn er ifm nbleght. 'Ein Elenber ift aud in biefer Ilbjicht, wie $\sigma_{\mathrm{e}}$ neca fagt, alb ein freilizthum zu betrachs ten, bá̄ nid)t Sebermann mit ijm fonn!ten uns walten fann, wie er will) fonbern są̧ man ihn fagonen, uno feinen ltingang, wie eine tofbare Seltenheit, genief̧en foll.Mein 2(rzt entoefte mir nach. Serlauf einis

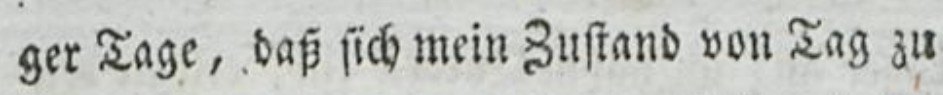
Tage verfálimmere, uno idh nithig batte, sum meiner Bienefung willen, allen sumult

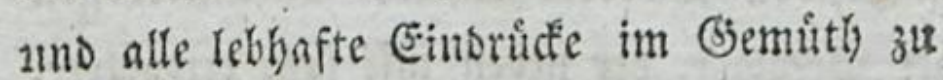
wermeiben, uno midh in cine heilfame Stille 3u begeben. Sal fapte biefen Entfoblus, uni fithrte ihn am folgenben Tage tapfer

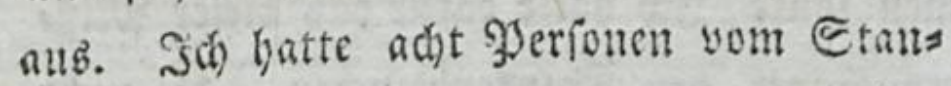
se, eine SBartfrall unb einen Эुrediger bey mir, als mein $\mathfrak{g t r}_{3}$ fam. Sut - fagte ich - Feerr Doctor, fetzen Cie fich bier; Feerr भुaffor, Sie bleiben bey mir; unb Ske 


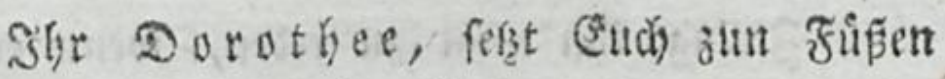

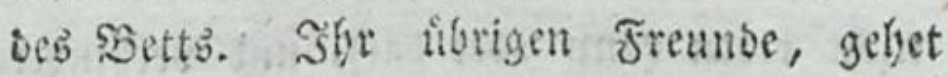
bin, uno betet firr mich; fo will id (5udb nack chigen Jagen fagen laffert, ob mir Dies mebr bifft, als Eure (Siegenwart. Disb war grobs.genug. arllein idj tam Gierauf in sie Stille, uns genas. Es: follte mir lieb fenu, wenn mein מeypiel 2nbere zu cben ber Dreifitigfeit ermunterte, bamit sie: fe fo befonertiche als ungereinte Nobe abges

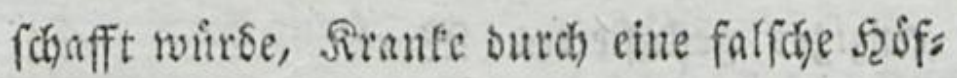
(id)feit on uerfolimment."

Cdyrake beu Siranfen Ridits vor vout

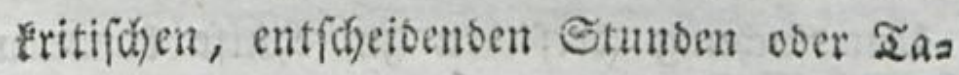

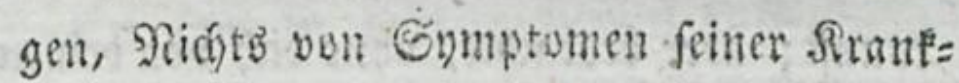
beit oder von sibirfungen Der \$rzmen, die erfolgen múffen, wem er gerettet mers ben fulle. Dab if grade dab ficherffe Shite tel, foldge glublidhe Symptome 4110233 it: fungen Der strzneyen zu verbinoern, uns ficon bie blofe soprifelfung bie er fich macbt,

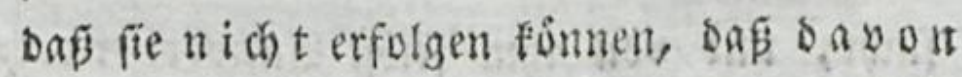
fein Reben voer Tob abbånge, tann allein ficon tobtlidy fur ign fern.

$$
\mathfrak{\text { Swing }}
$$




\section{2}

Sawing illu nid)t zu reben, wenn er nicht reben fann ober barf, unb verbindere es auf jebent Fall, baß̄ er nie beftig uno anlyalteno fpreche.

Fliffere nidit uno rese nicht beimlidy im Sirantenjimmer. Der Siranfe ift args

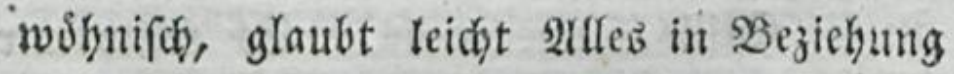
auf fich gefagt, mennt inmer, man rebe you

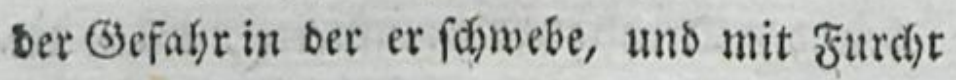
uno Schrecten erfüllt ihn oft auch mur ein eimzigeß gefluffertea 230 ort.

Sey) aufmerffam auf alle Symptome feiner Siraufleit, oamit Du bem $2\left(r_{z} t\right.$ bey Unterfud)ung berfelben zu frulffe fommen famnf; aber beobachte, obne bid yon beinen

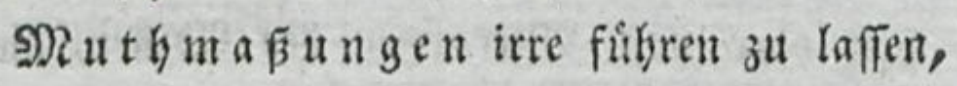
beobachte riditig und bemerte nut Dab, wab wirtlid zu bemerten iff; if ber $\mathfrak{S}_{\mathfrak{r} z} \mathrm{t}$ felbfi gegenwártig, fo unterbrid) ighn nidgt mit taufeno Sleinigfeiten und Rflbernheiten, wenu er ben 3uftand beb firanten unters fucht, und won ifm felbit 2(ntwort erwats tet.

(E)en 
(5ืben ba id) biejen 3 ogen in bie Drus deren fobitell will, fallt mir ber vortreffils che Untertid) für Kiranfenwàrter, *)

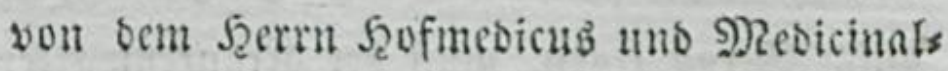

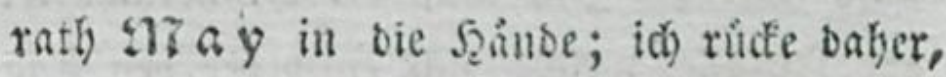
Do fo fegr vid bieranf antommt, feine 2 ha weifutg, wie der Lianfenwátter die Jufille der Zivantloeit beobad)ten, uno oiefe mit oen bemettiten 216 sin= derungen sem 2 trit befdreiben foll, bier :uch ein.)

„IIn Eeinen STanflyeiten ift bab fleigiz ge Bevbad)ten bes Siranfenwárter no nthiger, nls in jenen, weldse ifyer शature nach ben

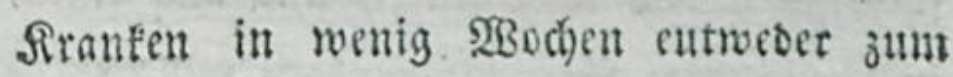
(Strabe ober zur erwinfobten $23 i e b e r g e n e j u n g$ fingren."

"Iีก diefe Reitge Sranflyeiten fann man alle fogenannte bişige Siranflyeiten fef̧en; ว. S. Giķige Bruffetantheiten, Entzinoung

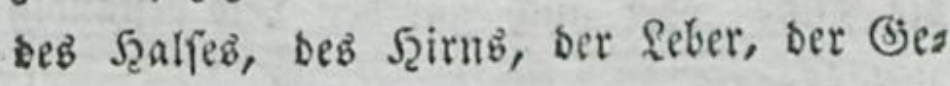
Dírme, ber Bebålyrmutter, ber શieren U. f.m.

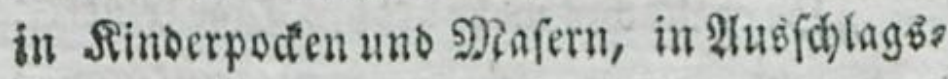

$$
\text { \& } 2
$$
fies

*) Nantugeim 1782 . 


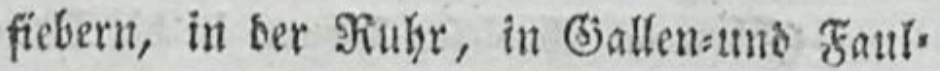
fiebern, weld)e sfters mefyrere $280 d$ en forts Dauern, uno ben firanten fo ganz langfain aufs Stroh Yegen, mup oer Siranfenwirter alle Sufälle, welche fich entweder bpy Tag

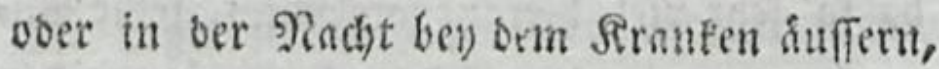
gennu beobaditen, unb sem 2 razt forgfältig Ginterbringen."

"Die in biefen Sitanfheiten zu keobad)= tenbe Erfdriningen unb aufälle fine forgenbe: , SOb ber Sirante ili ber Sacht rubig fiegen bleibt, fid nidgt im Bette bin unb bermallat, wimmert, wehelagt, uno mit ভeluns fudbt nach ben నag feufjt, unb aus einem in Dab anbere SBett Kinwanbern will?"

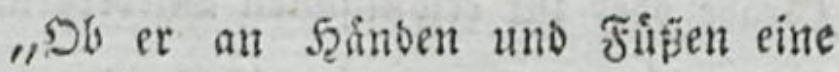
trocktre bremmenbe friģe bat, weiche beyn Infüblen cinem glihgenden Stein bernabe

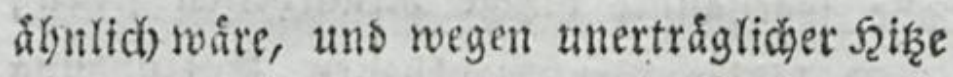
bie Funpe, oder aud) gar ben feib beftănbig entblofet?"

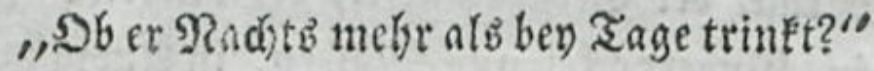
"\$b oer Siratfe im Salaf zulammen făbrt, bie 2fugen verbrehet, mit bem Namb allet: 
alferfand Betwegungen macht, of ifm ber Bart wackelt, ob er mit sent Bálynen gits ret? Biel unbentlide Dinge untereinander fdikitęt?"

,\$ob er mit ben Fingern beftanbig frabs belt, Hach eingebildeton Mituden jagt? Db im Edinf sie Selhnen der Finger aufipringen? Db oer Sirante wadbento afferley thoridgtes Selig (frid)t, nach ocm Trinfgefdirt zitterns reid)t, Darneben wie ein Blinoer greift, uno Daffetbe neben ben פiund ober an ben $3 a r t$ jum trinten anfergt?"

"Sb ex ben Szarn ind bett Stubigang unvermertt inb bett laufen láp̧t? Soburás Erbrechen voer ben Etnblgang $2 B$ uhrmer todt ober lebendig algehen?"

"sb er obne Sdanunbaftigeteit bie na, tirlidgen Gilieber enthropt?".

"Sb er mit einer troffnen burrten Bun= ge fein Trinfen begelyrt?"

,\$6 im lnterfeibe ein befiandige (5) ten unt Fumpeln gebort wirb?"

,Ob er befransig auf ben \&eibfungl bes gefret, aber weber Hurath wod Sarm you fich gibt?"

$$
\text { \& } \quad \text { n\$b }
$$




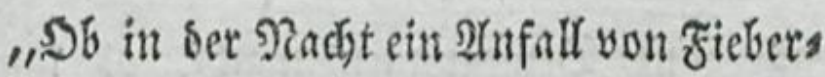
froft jugegen war?"

"\$b auf ein in ber Nandit erfolgtes शnfenbfuten, Enxiren wber auf einen liber Den gauzen Reib gehabten Edyweis der Sran= fe rubiger geworben ift, und einige Beit fauft gefchlafen bat?"

„Der Rrmfentorter mus ferner ges nall beubaditen uno betrachten, wab etwan Surch betl feufen, ourch bas erbrechen, ober sen Stuflgang, uno bey sibschnerimnen ober Sdywangern, sutch bie (seburtbtyeile abgebt - ben 2fusburf auffer sem Sirans fenzimmer aufbewabren, uno ouf Segehren sem $2 \mathfrak{r r}_{3} t$ vorjeigen. Eben fo fursfáltig mus ber nady Mitternacht gelaffene Ltrin auffer bem Sranfenzimmer in cinem reinen (B)โав alffewabret werben."

"Sullten bey Tang ober श(ac)t bey bem Rranten fich ganz unerwarte Sufülle, $; .25$.

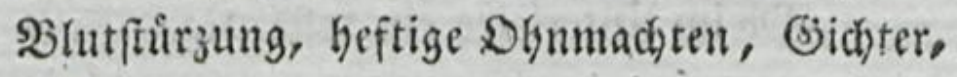
Starrfucht, blutiger Stulglgang, \&afhmung, auferoroentlide Geftige Schmerzen an einem

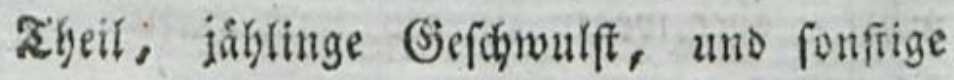
gallz 


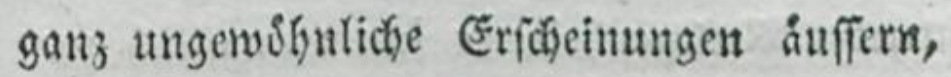

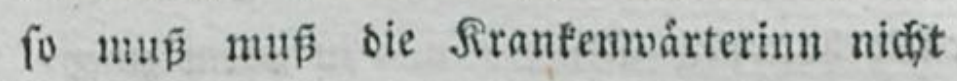
Den Zang abwarten, fonbern bem $2 r_{3} t$ foldge Bufulle fo gleid eroffunen Iaffen."

"Ileberhaupt mus ber Siranfenwarter Dab rebensige Tagebuch aller bey bem Sitan, fen cricheinenten Bufalle feyn; befonbers jene, weläje fid nádgtlicher $2 B$ eile siuffern, forgs fâlttig allzeigen, wenn er feinem Brewiffen (j) núge leiften will."

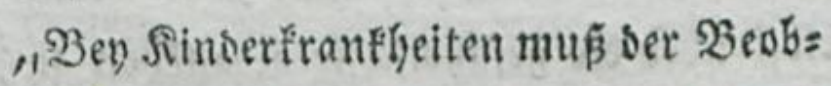
ad)tungsgef famer uno thatiger feyn, weil bie finber ihre. Siraufbeiten niđjt beutlich erfláren fơmen." - - -

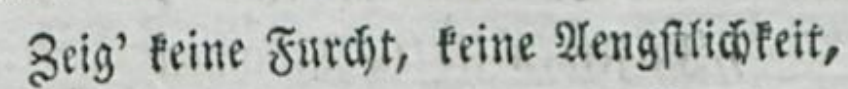
Feine Beforguif am Bette Dę Siranfen;

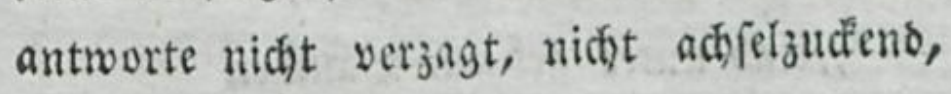

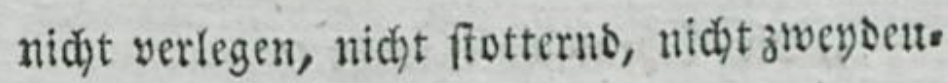
tig, wemn er Dida liber feine Siranţeit frágt, wenn er von Dir husen will, baje bu nodh

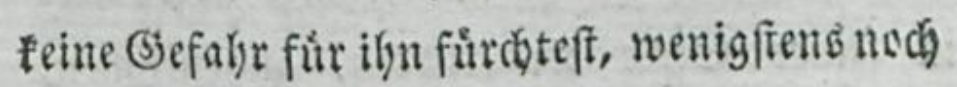

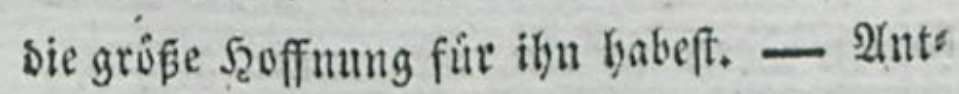
morte rafch uno fefr. Sin jebem Stottern, $£ 4$ in 


\section{68}

in jeber Şerlegentheit, in jebent angfflich jus ridfgebaltenen Senfier und in jeoem zweys Deutigen Befidst birt und fiebt ber Rranfe faft inmer fein gewiffes হobesurtheir, uno war feine Serantyeit vorbin nidyt tobrlich, fo miro fie's sam gewín.

Spridi ifin immer S?uth uns f̧off= mung ein, ourch 2Bort uno 23 ficf unb Giebelys: be. Rebendige Soffinung wirft oft unenorich mebr, als bie befte 2(rzuey - faun oft felbft ůker bie gefäbrlichfie Sirantheit fiegen;

"Sie ift $e \hat{b}$, die Dem Sirantem

"Die Tasesqualen ftillt;

1) Pit monnigen (jednnfelt

"ภ̊on Butunft ihn erfüllt;

"Sิ่n feinen leşten నräumen

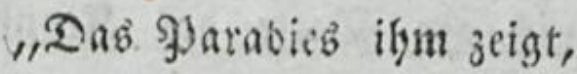

"Uluo unter grinen 3 åumen

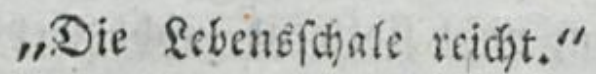

23 unger.

SBecte, belebe uns unterbalte ubberbaupt in feiner Seele ber leichten, angenelymen, beitern Soeen fo viel, als immer moglich; zerftreue alle Furcht und alle Bangigleit in

ibnt 
ifin, unb bebe ifin jut einem Snuth cmpor, ber alle befalgr aub bem. Ituge verliert. ")

2lber willft ou ifyn Nuth uno ßers trauen cinfpreden, willf su frimen silauben

a)t

*) NBie mefir die Secle des firanten bie

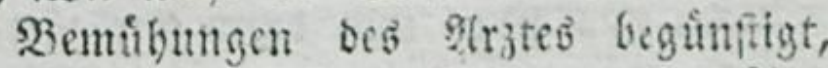

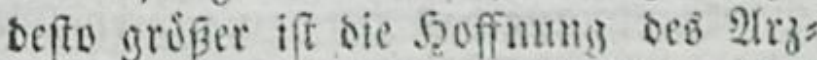
ter. 28 ie megr Einflup die gieden Des

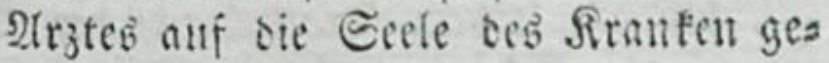
getvinuth, befto ridstiger foun mat

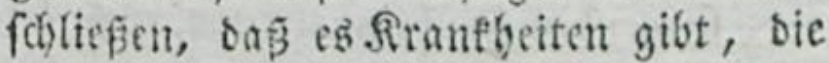
fiid) Durch sisorte linbern faffen. Dies for Edhlus ift anf bie Erfabrintig ges grimbet. Stan weiß, wie $23 i c t$ in Ges mútlyefrnebeiten auf einen 2 rrat ans $^{2}$ fommt, Der Futhe uns \$ergnilgen in allen 3eiten zum Dienfie mitheidswers ther Siranfen unterbricht; ber eb̂ fur

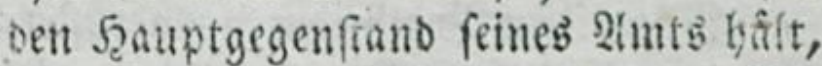
ifgre Leioen ju fúblen; ber bas Ge= muth bes Siranten nach allen feinen gBirfungen, uno biefe nad) allen ifren Lirfachen burdfortngt; ber biegfam ge= nug ift, immer nach ben llurftanteent mit Dem firaneen zll reben und jul bans Deln, feinem Elend fić ju unterwers fen, und fein fleimmithiges $2 \mathrm{Befen}$ zlt vertragen; Der es in feiner Bemalt

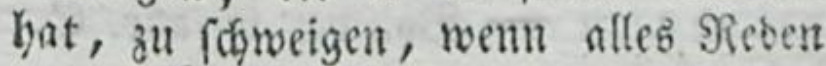
umfonft iff; burd) Sanftmuth ber (sies

mút 
nu Bott beleben unb erwirmen, - willfit sul ifn bey feinen Sdymerzen mit Geraffen= Yeit, Ergetung uno Sindlidéeit erfullen uno ifn mit gortlichem Sroft ftäten aus oer Ses

gent $=$

mifyt jut jwingen, wenn alle Etaitfe fraftlos ift; uno ourch eole zartliche

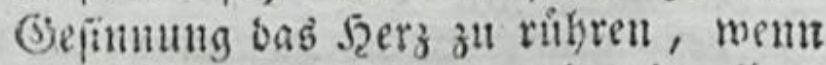
eb biefen Gefimmungen, wie bie allzu= lange mit beut Trouterfleide bes IBBin. terb-ambangte Erbe ber jungen auf, freigenden Friblingsblume, fidi) offnet; Der enstidt an Der SBrute ber g)iufen

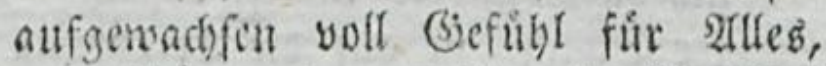
was fchún uno großs iff, im Notbfall feine QHufmunterungen Dutd) bie 9Rad)t einer eimelnmenben Dereofantét uns terftiben, unb burdb alfe Simfte einer fobnen Énbilbungseraft erheitern faum. ) Rit biefen Bortbeilen auggeriftet bifft Der 2lizt bem Siranten feimen Slórper iberwinben, uno er füllt feine Seele mit Sanftmuth, mit Jeffinung uno Unerfdrudenbeit. श्tle श्trmeyen fins in foldben Fitlen umfont, wenn ber

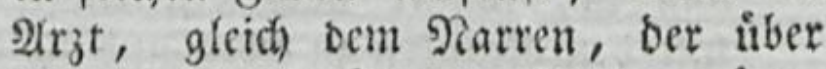
$2 B a b r b e i t$ und Tugeno fpotten mogte, jene nufzlluchen zu faul, ulto biefe

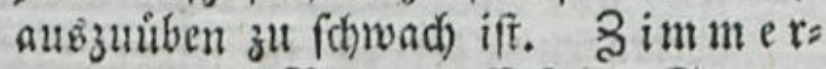
man $n$ Son Der Erfalir. S. 259. 260.

श्रî: 


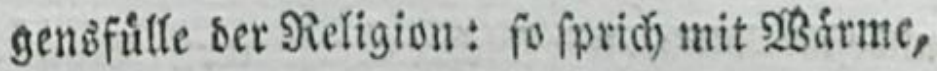
mit Heberjengung aub vollem úberftróment= Den 5 erzen, ober folweig ganz davon uno verfindige Did) mit feiner Sylbe. Es if

IIns

2iiemano fam you ber SiBabrbeit siefer Sermertung imniger niberzengt fer)n, uno siemanb fann fie mit fo viel 3tuerficht uno शadebrud empfehs. ren, als eben ber grofe, eble Siant, aus beffen unferblidem $\mathfrak{B B e r f e}$ id bie vorfethenbe Etelle entrelgut habe, weil genis शiemanto auf bie yon Shm bier empfoblue grofie, fogwere futuft fich befier verfecht, uno siemano báufigere und auffallendere Exfahrungen yon ih=

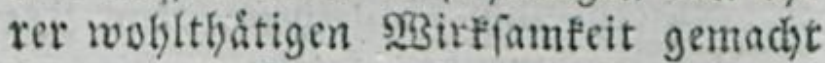
hat, alv Err. - Ewig unvergę̧lid) if es mir, wie id Shy einft oft am Ranenbette (Fines meiner liebfen FrennDe reben burte unb hanteln fab. Er fant zu Diefem zumerftemmal, alsihm

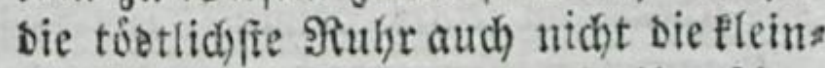

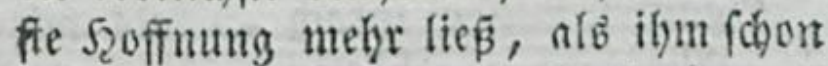
alle \{ebenzfraft ganlz abgefturben $z^{\text {it }}$ feyn fohien, als ibm bas fürdsterliăfe

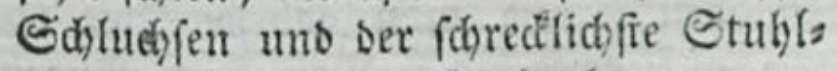
jwang, was fäon 2sodjentang umuns terbrodien fortgebautert batte, Feine Secunde lang Siulge lieff, als fdjon

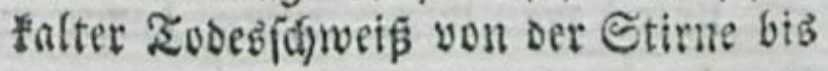


mbeforeiblich, wie forcher Troft jesen frrans fen, jeben Seibenten etquidt uns erhebt' nent ef alts einem roarmon, yollen, cblen Serzen mit funftofer פerebtfamfeit in bas

feinis

zu ben Fingen aus ifun orang, uns alb fein bisheriger febr gefolicter, fehr erfabrner, unermideter, trefflicher 2lrst relbfi alle joffuning oabin gab

In biefen für mich fo forectlichen mutblofen Stundell fam $3 \mathrm{imm}$ ex: mats n, uno o! wie waro nir, uno wic warb meinem ferbenben Freunde, fhon in, ais wit Sign nur vor unb faffen! - 2tlo wir bie unbefóreibliche Milde uno Sanftbeit, Dab $2 B$ arme, Sheifnelsmenoe, feerzolle, (Sutmutbige 3utranngebende, Butraunbinteiffende, Rebenvedende uns Rebenfiarfende, was 2llleb aus jeinen Augen voll $\mathrm{Se}_{\mathrm{er}}$ uns Seele, aแb jedein Eeiner 2 borte, aus jesem כon feiner Stimme, aus Eeinem ganjen Saben fprad) - alb wir bas Zuleb faben, borten, füblten! Saan mu B te S6m ¿eib uno Eele binger ben, uns man gab fie Şbm freubig auf emig. - Şon Dem 2ugenblid an febrte auch ber Sterbenbe inb $\mathfrak{L}_{e}=$ \& ben zurud. 2lle Furdit, alle Mutblo= fibleit war weg. SRan Daste meiter 
feinige bringt, went bas 2 gort จ

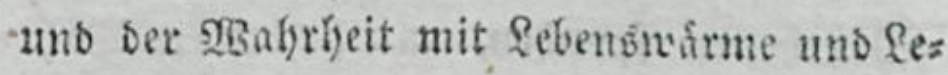
bensfraft frin ganjeb SBBefen crgreift; wie

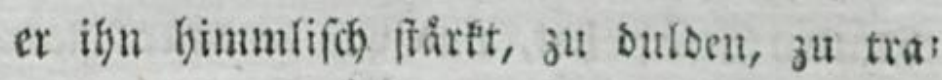
gen, ju beten, zu boffen, felienfeft zu trauen auf ben (Sott, ber ba bilft, ber felleft your Tube errettet. - 2 tber $e \mathfrak{b}$ if and unber fdreibfich, wie Richtswirtent, wie wibrig, wie empórend es ift, wenn cin falter, elents Der

an Feime Brefabr. 2Hber fo batt' th aud) nie Natth einfprechen horen, nIB Er eB that, uno nie babe ich folche 23Birfung bavon gefetyen, als ich bier fab. MRein guter, theurer gु ** um beffen Genefung ids felbf nidgt mebr zu beten wagte, weif piefo ganz unmiglid) (d)ien - waro gerettet, uns

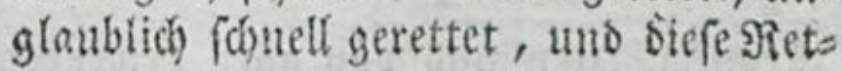

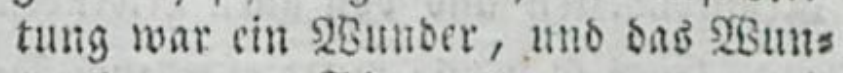
ber fonte mur 3 i m mer m a n $n$ mit Bott yerriden, weil Pliemans, fo wie Er, Menfogenterzen zu beleben, uns

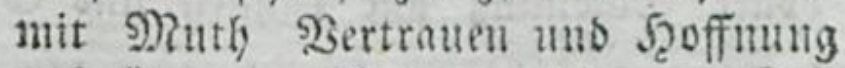
auf Gott zu frátfen weis. - Eeit

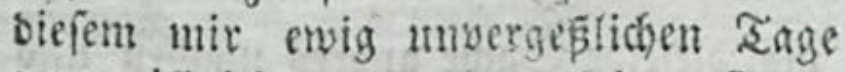
verzmeifle id) mun aแc) an feinen firan: fen melyr, fo lange idf noch $\sqrt{2}$ off $=$ nung uns 9$)$ ? 4 th ben ifgm rebenbig erlyalten fautu.

23. 
Ser, erbarmlider SRenfids von foldsen SBabrbeiten fprechen, mit foldye n 2 babr: beiten troffen will, für bie cr gar feinen

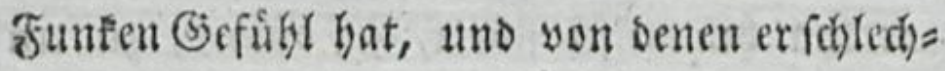
terbings nidst fprechen famn, nidet fpredyen foll, nidbt fprechen Darf. Snz Ingeficht băts te idi) oft foldben Sieifreblabmen, berzlofon, edelbaften Sudffabenmentchen ifyre Gaalbas ocreyen fpeyen migen, wenn idg's in jebem

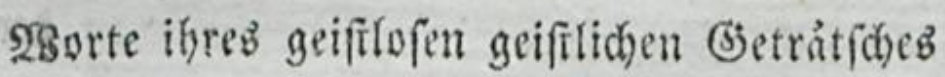
uno in jebem Ton ifrer bớtzernen Gtimme unettríglich füblen muste, onв fie ba blof

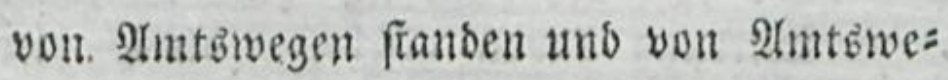
gen flofinten, uns baun bolblelig iffr 2lect. sens einfrichen uno rubig heimgingen Ent: ferslids! - XBeg mit Soldjen yon allen Sranfenbetten uns yon allen feibenben!

Eben fo wenig taugt aber aucb Der bey Siranfen, ber nicbt mit Giebuld uno liebe: volfer Schoming alle Launen uno Griflen. Des Siranten ertragen fann; ber nicht zelon mat biefelbe Sache anbers madien unb fich's sanu nicht auch zum eiffen mal noch. fagen

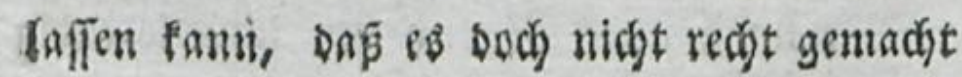
iff ; 
iff; ber nid)t Sanftheit uns Biegfamfeit genug bat, um fids ganj an ben Leibenbert

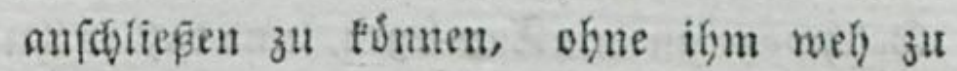
thun und obne fid von ifun unertråglid ge: brufft zu fúblen; ber feinen feinen Ginn bat, um ganz in bie Geele des Siranfen ein= zubringen, um alle feime Empfinoungen zu verfteken, und alle feime Blicte ju beuten, uno faft alle feine SBedurfuiffe ju erratlyen, ano unjåblidje Forberungen uno Errvare tungen ungeforbert zu befriebigen; bem $\mathrm{eb}$ an Delicateffe uno Befuhl fehlt, sas er nidyt Alles zu ber Beit, allf oie 2rtt, in oem Raafi, mit oem SEefen thun fann, wie eb Der Rranfe getfan wiffen will; Der fich bey ibm nicht gams vergeffen uno ifyn niäbs immer ganz in Shuge bebalten famn; Der

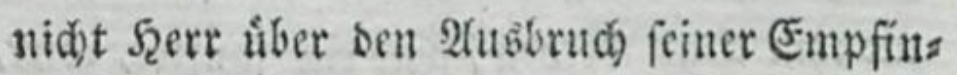
Dungen uno ûber feine SRinen iff; Der feine 3unge nidyt im 3aum balten mo feine S(i)nadfudyt niợt banbigen fann; ber ba meynt, Daf er feine Ibeilnabume an ben \&ei= ben bes Sranfen nidbt beffer uno nidst ans

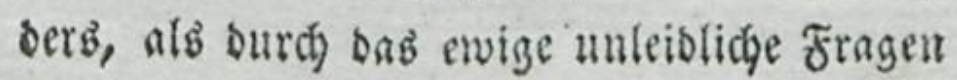
"twie 


\section{. $\quad 176$}

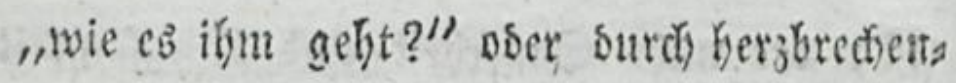

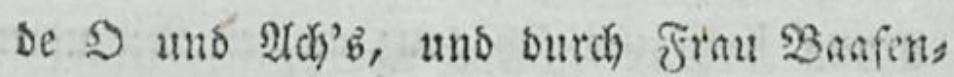

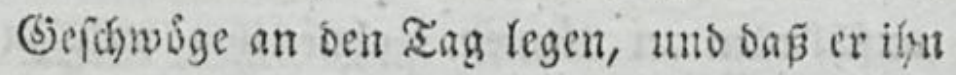
nicht beffer anfbeitern fómne, nls wenn et ifm you Morgen bib zum 2 beno uno yom श्tbeno biв zum Sourgen wab yorrabbelt; ber

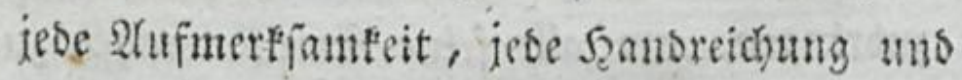
jebes Eleine Spfer, was er fenter (S) emáchfide

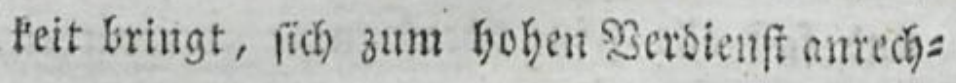

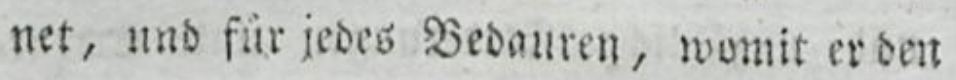
frranten quâlt, wieber bebauert fern will -

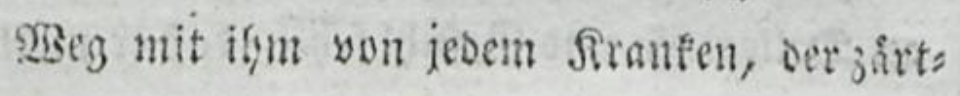
fider \$y frege uno liebeyoller Edyuming bes ourf; er wirb nie feine sdymerzen linbern, nie fein feisen igm erleidtern, nie ify zunt

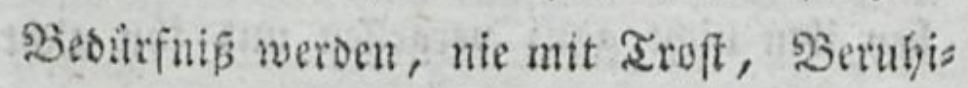
gung Erbeiterung uno Muth ign betcben, uno nie ihm Dab werben, wab eih fanfter, esler, theilnebmenoer, wohythátiger Siranfenpfieger Dem firanten if uno fenn mus ! -

\section{II.}




\section{II.}

Jtaftiffte $\mathfrak{B}$ eleftrungen und Erinneruns gen für Die, weldhe mit Eecidenden umgehen sollen.

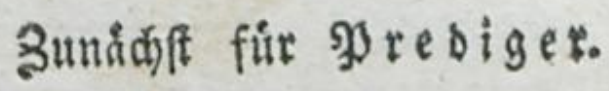

$$
* \text { * * }
$$

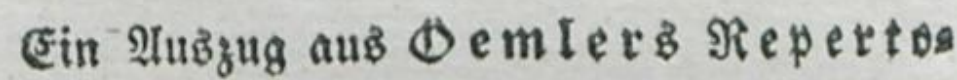
rium úber \$aftoraltheologie und Eafuiftil. Șena 1786a

1789 . 


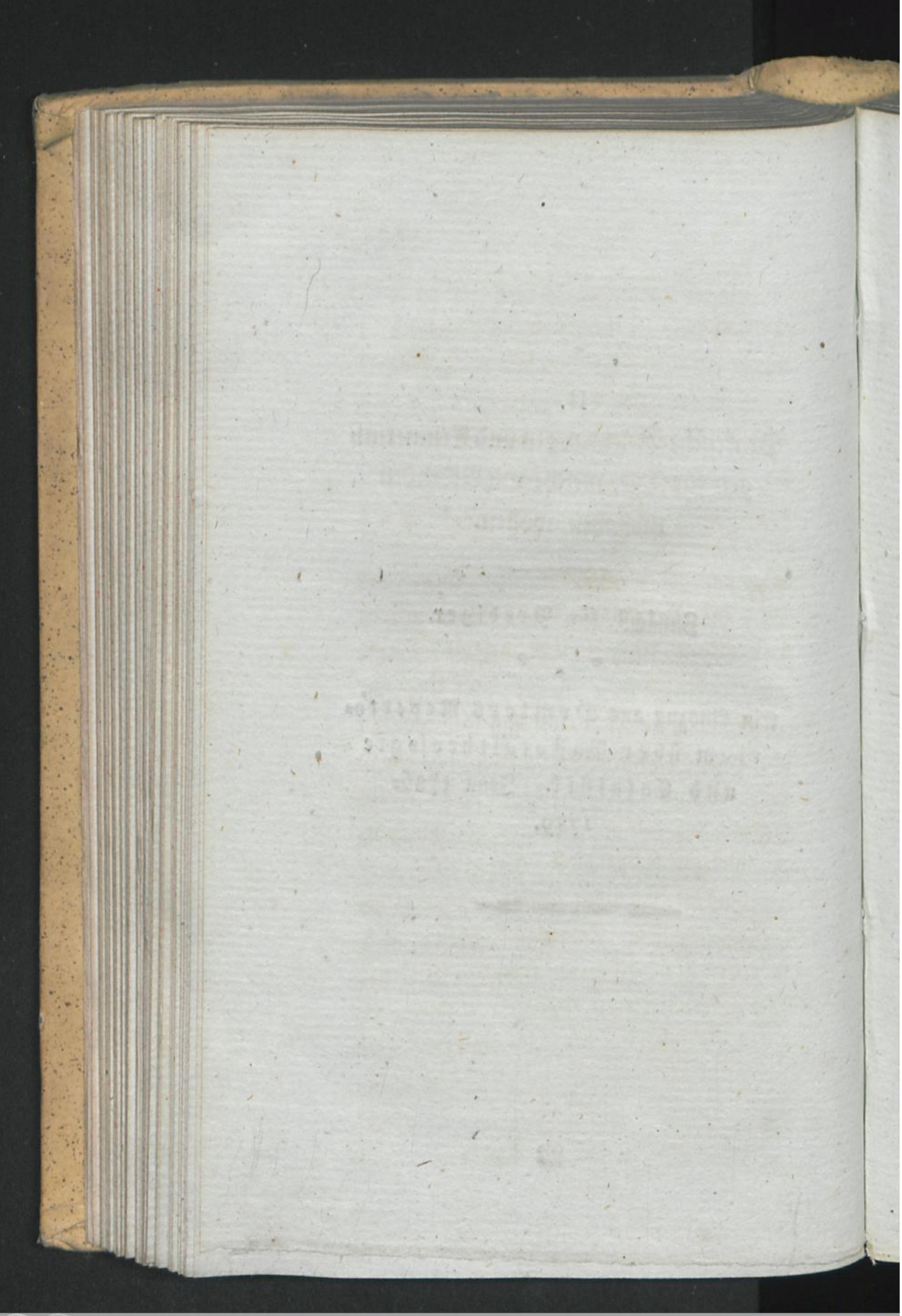


Oraftiche Sbelefrungen und Erinuerungett fur Die, welche mit Reibenden ums gehen wollen.

I.

Heber Slyeilnet)mung Des Serzens. *)

S) 1 idfts if einem भुorebiger in feimem 2 mte nútbiger, als ein freund frbaftlides, liebretches und theilnebmentes bet5. - - - Sidi bat die Erfahrung getebrt, dap es Dem, ber fich für mich) ins terefijren tann, unenblid leichter gelingt, etwa úber mein $\mathfrak{J}_{2}$ rz z̆ yermógen, alb einem 2noern, der falt uno feetentos vor mir felfy, wie viel bie Sunfi aud verfámente. Das ber muв es fich) jeber ßrebiger zum erfert (3rturogefers feines umgangs machen, fid mit den frò Den Zetruibten 3 weinen, uns bey bem erfent Anblice, bey ber erften Bufprade, eis then sBlid in fein J̧erz entweder yolf fress श 2

bers

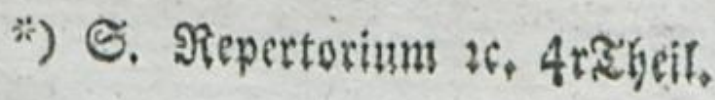


ben ober voll Mriteio zu thun, ie nadsem or feine 3ubjorer in einer gewiffen lage ans trifft.

Der Traurige uno Betriote foll eร auf meinem (selichte lefen, bap ich ifn bebauts re, und er fulf es Darum lefen, meil id es wirtid thue, weil id $\mathfrak{e b}^{-}$mir vorfagen will, wie erquideno es mir im gleidhen fals le fern wtive, wenn id ein Ferz, bas wit mir füblte, fånbe. Er poll eb merten, Dafí mir fcine Rlagen nidgt zur laft fino,

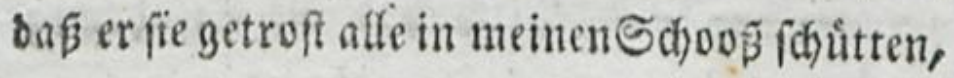
snid) mit den fleinfen Detail feiner Seiben unterhalten barf. (ङr foll Ruth) zu mir faffen, foll - wie ein gutez אino; oaz, fobalo ibu etwas ift, zu ber lieben Matter, eift, unb es ifir warm uno beif, wie es fuiblt, fagt - ald mir jebe গiegung ents

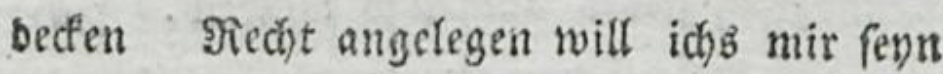
faffen, fo fanft, fo freunolid, fo voll siebe mit ifm umzugetgen - Das foll ber erfe 2:alfam feyn, ben idy an feinen $2 B$ unben ver

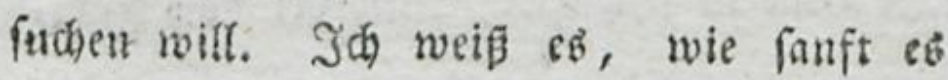
thut, wenn man vou stlen verlaffen zu

feyn 
fentt glaulit, unbeine eble Seele baun fommt

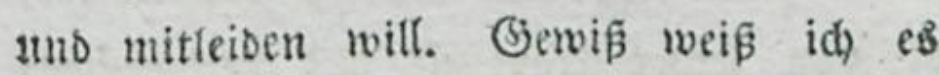
aucis), ber Eeibenbe, mit bem id fo umgehe, wirb Erteidterung finden, und ber Sdmerz, Den er vorber allein in feinem $\mathfrak{F e r z e n ~ v e r e ~}$ fosfiepen muste, uno ben or num mittlyeis len, attỏweinen fann, wirb fich minbern, uns bie geprefte 23ruft wirb \&uft befom. men. - Ján werbe ibm lieb werben, er ntro meinen lumgang fud)en, fid) nach mir folnen Die rasorte, Die id ifm dann im Namen meinez feerrn, ber audo, alg er auf Erben oie Shubfeligen 孔 fid rief und - bie Traurigen getrof feyn biefi, finge, werden ifm 3 Borte eines Engels fern. W3ab oarf id mir nidt won folder zufpradje verfprechen! Dies mein theilnebmendes Fets $_{3}$ flóft mir bie Eprade ber liebe ein, uno legre mir $\mathfrak{B}$ orte in ben gaunb, die bab trau=

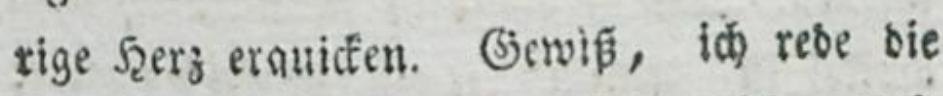
Spradye des Freundes, nad dem Sinn meis

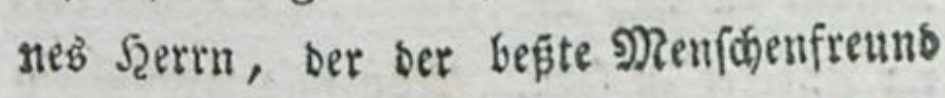
war. Fin siefe $\mathfrak{B a f b r b e i t}$ reset die Ers fabrung laut. Selbfi ber gefiagrlidfte Srants आR 3 fe 
Te empfindet bey sem 2lnblice bes $2 r_{3} t e z$, ber yon feiner Sranelyeit mit theilnebment= Dent $\mathfrak{S}_{2}$ rzen reset, einige Erfecichterung. IBBie notthig ift eb alfo, ons fich Seber, oer fith Dem भुoréigtamte wiomen will vorbereis te, um einft ein máchtiger Trúfter, ein willemmner Freunb ber $\mathfrak{B e t r u ́ b t e n ~} 3$ wer:

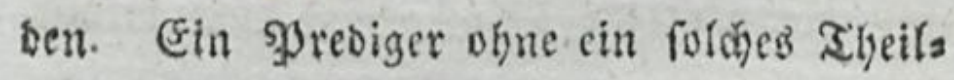
nehmenbes $\mathcal{S}_{2} \mathrm{er}_{3}$ ift fein wahrer evangefis face \$rediger. Llutb boch, wie viele unter นแถ Gaben harte, unempfindridge unb gefithts Ieere Seeten!

\section{Wie bereitet man fidi) aber baju}

\section{vor?}

3ิuerfi, mein Bruber, bu gewsinnef Dids fobalo als migfid daran, Dir ein an $21=$ Iem, was bie Menchibeit angelyt, theilnely, mendeg, mitempfindendes $\mathfrak{S}_{2} e r_{3}$ z̆ll ers werben. Dab if ohnebem fdjon ein $\sqrt{2}$ autpt= artifel in seinem menfacnberuf,

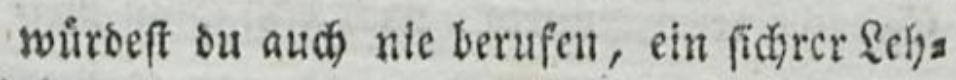

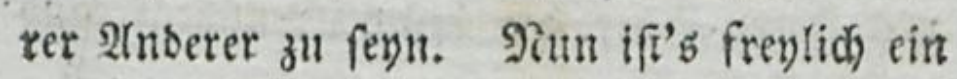
Foffibares (s)efhent bes guten uno weifen (Sots

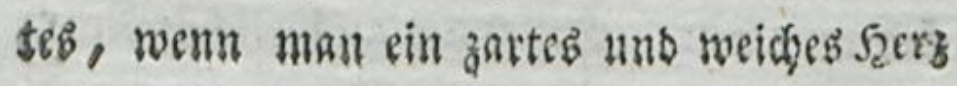

Gat, 
hat, uno er weip am besten, warum et aud Diefe Babe nidgt im gleiden Draage yertheift hat. - 2tber Dab fag' id boch

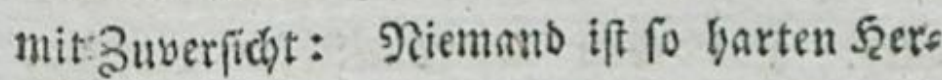

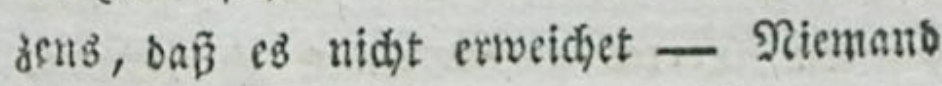
to in fich verfoloffen, to in fein eignes Sclbft verliebt, ber niđ̆t ber ausfiépenbfie, theifnebmentfie, allgemeinfe Nenidgenfreund twerden finnte.

Madje baher gerviffe Oedantelt, bie

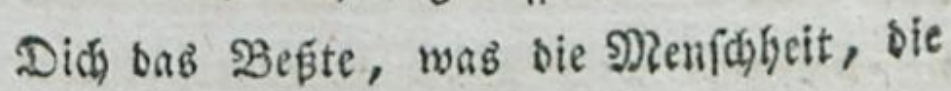

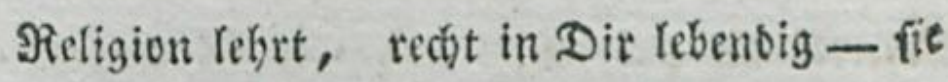
werben Dids zur Nenfohenliebe erweden. Sic nun oft liberoadbt, fie in einjelnen szorfallenbeiten angemendet, ba fie fich vou nettem vergegenwarrtigt, ba nad) feinem nod Falten Befüh! jugewendet. $D$ - wie wirs eß̉ erwármt, wie angefenert werben!

Im 2Infange 'migt' id frettige $\mathfrak{B}$ egeo benbeiten worfaflagen, an benen wir Theil nehmen lemen follten. Dab \$Bergnúgen iff weit in die Sinne fallenver, fid mit 2 ma

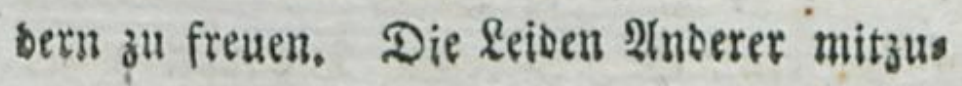

\section{श्य 4}

ents. 
empfinden, if fibon eine feinere gुobilluft, bie bab edle frerz zwar, bat eb fie crinmal

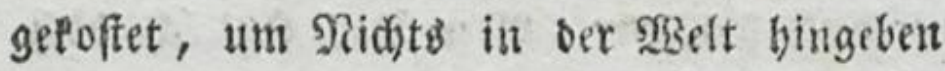
wirb. F̧at mait fich nun erf gewobnt, Freuben Anberer mitempfinten zu fonmen, nic)t uur Freuben unfrer Freunbe uno $\mathfrak{Z e r}=$ wanoten, aud Freuben unfrer Lubefannten, 2abwefenden, ober gar unfrer Feinbe: to wird es nad) und nad) f(b) dem Şerzen nicht blof mơglids, eह wirb ify unentbebr, lich merben, auth Sigeil an bem leiben 2lnoerer zu nekmen. 2rber eb if freylid) Sichts in ber SBeft leid)ter, als mit einer gemiffen Empfindfamteit groß zu thun,

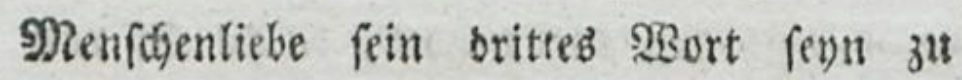
raffen, uno babey gleidgwohl fo unempfinds lid, , fo untbåtig als móglid) zu bleiben. -

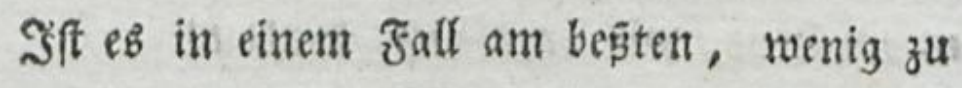
fagen uno befto Mefir zu thun, fo ift eb

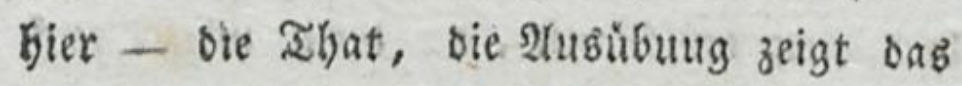
gute, theilnebmente f̧erz. (Berosfnulich) fprecken Die aur wenigften yon Menicken= Viebe, bie nicht gerne einen Tag borbey ge= 


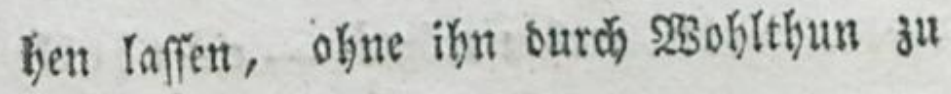
bezeidonen. "*)

Dies wáre bas Erfte, was ich Dem, ber fidg ju bent Ltmgang mit Reibenden yor=

\section{ఇ 5}

berei=

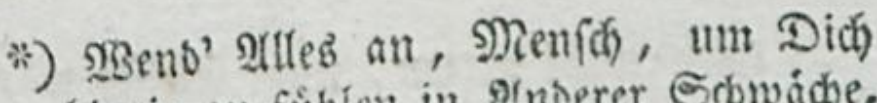
bincin ju fiblen in 2 Inderer Sdywadhe, Empfinolic)feit, Englumigteit, in

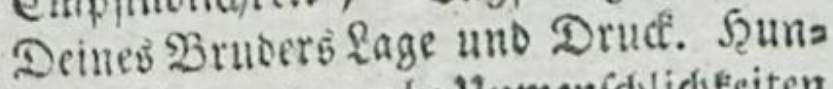
bert feine uno grobeltmmenfdyliclféten merben blö́ Debrwegen begangen, weil man fich in $b e b$ ?tnoern Lage nidgt

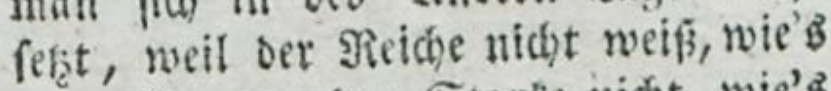
Dem 2trmen, ber Starfe nicht, wie's bem Edwachen, ber Gejunbe nidst, wie'b ben Sranfen, Der Hnempfindlicte, Linreigbare nid)t, wie's bem (smpfints

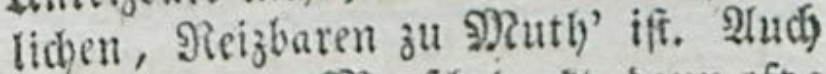
Ber fonft. gute Nerife dentt baun oft: wab if m nidt wely thue, mads' aud bem Indern nidet weh, was er nidit beourrfe, beourf' audd ein Inderer nidist; was if m nid) tbange made, i h m Ileis

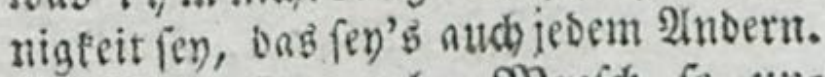
Drandem if mander Menich fo uns Deocutend, Dá̧. er gar nicht baran denft,

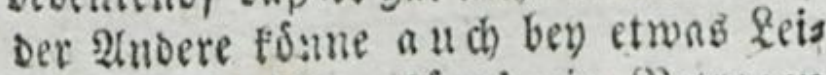
sen; aud ifim 'fonn' ein Sbetragen weh thun. Ilnd barum befjandelt er Sinter, Titebrige, şediente, alz 06 fie nichts frantte, als ob fie fein Nen= icherts 


\section{6}

Gereiten wollte, rathen mogte - ein the its mendes $\mathfrak{F}_{\mathrm{C}} \mathrm{r}$.

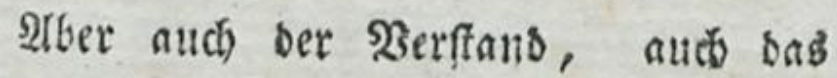
Radjoenfen will feine Befbdftigungen baben

baben,

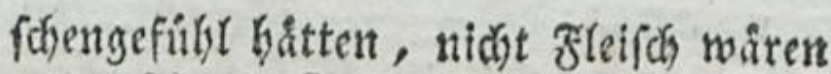
yon fcinem Fletión. - Berwabre Did, Menfd, yon biefer E i nfoitig, feit unb Selbft $u d t$, wenn Du menfd)lid) bleiben und menf(tid) wer: ben willf. So oft Du eannft, made

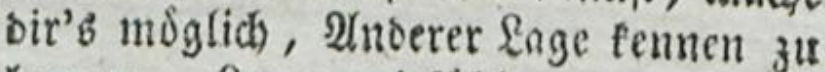
Yernen. Eerne mitfúblen den Drud bes Bebridéten, die kengftidifeteit bes Slengfilidon, bue Edomádhe oer Echmas den - Bleibe nicht inmer in Deinem

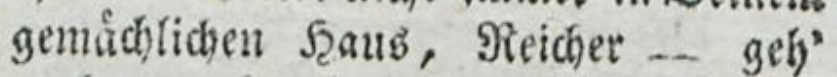
audh manditual in bes̉ Armen Scutte, Damit Du fiebft, wie er lebt. Eieb nidjt immer Bsefunoe um Did, geh" audi) cinmal ans Siranfenbett bes Rrane felt, Danit Du fúblft, wie er fédmadys tet, was if $m$ ein burdfeufater sag, $^{2}$

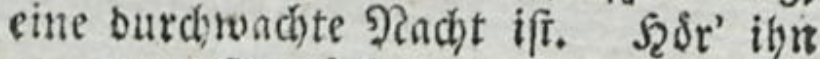
an, ben Georiditen, wenn er Dir finten Drud tlagt. Du wirft in not ein Slluge baben, bas bie Szabrbeit feiner feisen verfeft ; uno wann Du SBabrbeit in ihm fiebfit: práge Dirs" tief ein, wodurch er leibet, obgleich Du sicf)t babey leibeft; $\mathfrak{w a b}$ ibn

oricts 
Saben, uno fie vereinigen fith alle in bem Einen, Gtubium des menfolidyen berzens. So wenig man ofne dab ein guter Rebner werden fann, eben fo renig,

brificte, ob c Lerne burdh Humgang ben Edymadhen verffefn in feiner Schwadbeit, Det 2lengfrididen in feiner Furd)t. (Gib 2(d)t, wie leiỏ)t Diefer oocr jener MRenich ges sricét, gefrånt, befeisigt werben fant. Denfe, wie Dir's fenn wirto, wemi

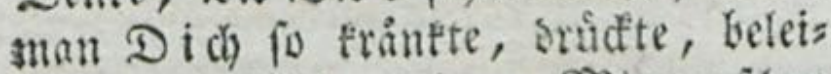
sigte - fuble beinen Mitmeniden alb Dein Flleifo.

Tisu' aber auch 2tlles, 4 m totits Ieioen uno Ditgefơb 3 u erbalten in Deinem ber3en. - एङ ift leiber

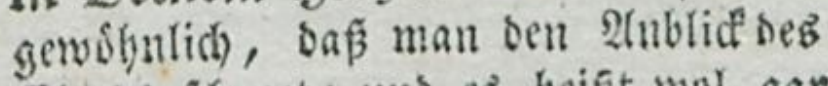
Elento fosent; und es beist wol gat feineb, tiefes Gefáty - Empfindfams feit, wern man ifhn fieft. - Ihu Du bas nidt, lieber Menfa! \&a官 Elenb jeder 2Irt Dir labe fommen.

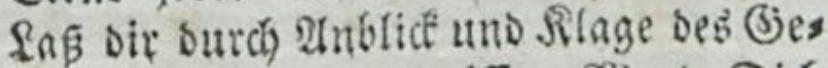
Drúdten bas feerz zerreiffen. TSEenbe Dich - nidjt weg, ent

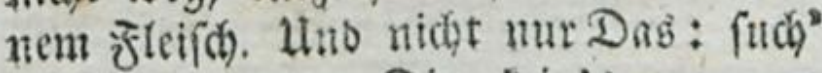
ez nuf, wenu Dir tein's entgeger fosmmt. (jeb' зu Dem Lager des Rrants Fen, zu bem Sdywadien, इroftufert bitt. 
ja nod) vicl weniger, wito utant ein guter

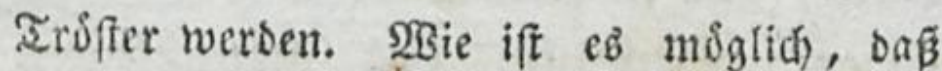
ich Eingang in bie Seerzen finbe, wenn idy bie $23 e g e$ nicht fenne? SBSie ifto miglich), baf

bin. Sefuche ben Armen, ber faum Sirobt hat fir fich und feine Sinder nidt um Deines Srubers, fondern um Deines Seerzenb willen. Utnd ničbt blos, wenn Du belfen tannft! $D$ Dann ift's Frente binzugehu zu bem 21 rmen, dem אiranfen, hiddfe Mens

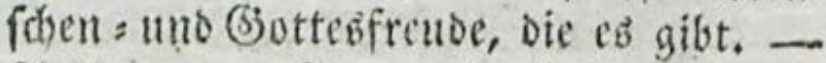
Ind) wern Du nidb t belfent tannfr, meide den (Elenten nid)t! Diefer Drang in Deiner Bruft, Diefe 2ertegenbeit, Diefer Edhmerz liber eigene Dhmundbt ift, wie mandser Edbmerz beym Sint, Seichen bes 2 Bachsthums, 2 Bedjel auf bobere Macht - SBgie vortheilhaft fint \&eiben von biefer Seite! wie ers weidsen fie, vermenfditichen fie unfer 5ers! Echwerlich gibts einen recht grofen, auch) von Seiten Deb F̧erzenz groß̧en Menfden, ber es nidht burch Qeiben worben wáre! - Dod) nicbt blof Equen, fonbern aud $f \mathfrak{r}$ с u o en Deines פrubers fúble mit. Und nicht blo Freuben (sinerley2lrt, was etwa such Dir Freube ware. Sief', wie'z bem \&nnbs matn bey finter Silthe nad 2trbeit, wie's 


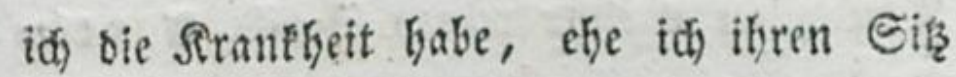

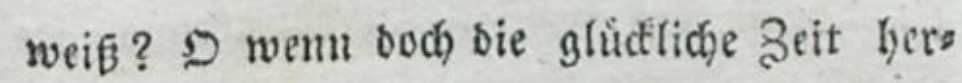
einbradge, wo oie Menfden mehr anfingell, liber ben Menfdien zu benlen! 2Beld Sidht, weldye Etrablen von allen Seiten! weldye Erleudstung in taujeno Morfallentheiten! Jun wiro erfi ber fefrer wiffen, wie bie 23 unben bhten, bie er beilen foll, uno fein meni(d)enfreundfidgez liebevolles̉ Şerz wiro ben gróşten Theil an ben feiben ber Briber nebs men. Die Semeinde ifi ju betlagen, web de teinen Rebrer mit einem theilnefymenoen Szerzen bat, beun ein Soldher fajbipft feinen Troft wie's ben Sintern ber ifrem Epiel, Dem Seinfinnigen bey Naturgenuß, bem Ebefn nach einer guten That, Dem $23 i \beta^{2}$. begierigen nach Erfernung einer netlen Sumft, bem Liebevolten, wenn it Freube madite, bem (Sottegiverehrer nach Gefúbl Der शakhe Gottes fo woht ift.

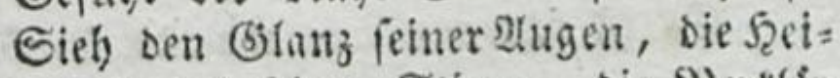
terfeit auf feiner Stime, die șertlá= rumg auf feinem 2(ntlif, bas neue les

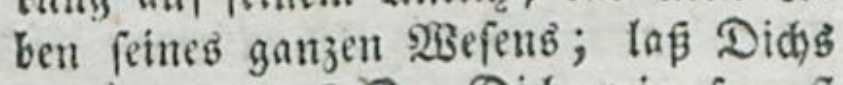
entzünoen, dáp Dut Did) rein freueft Der Frente bes 2 Anbern, ber Fleifch if yon Deinem Ffeifá.

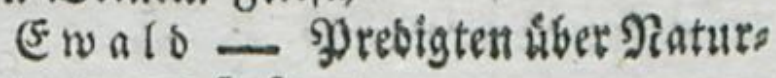
texte. 28 J 5 eft. 
Troft nie ats einer reinen Suelle. 2tus bis: fer Suclle bes wabren Egriffenthums ges fchopft! Diefer Troft frâtêt, er betébt, et

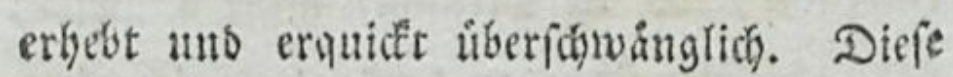
Qutle wiro nie auggefdipft, quillt bin= fiber in envige Scben, hat Labung fur alle 2rten yon Reibenben! \$lein junger \$ruber! entziche Did ben Sgutteu ber Etenden, der (Gebrifiten uidgt! befuctie fie oft ! befuche fie gern, und gewólye Didf von Deiner Sus geno, Theil an ifrem Elenoe zu nebmen! So wirft Du cin walyrer evangelifocer \&ebrex

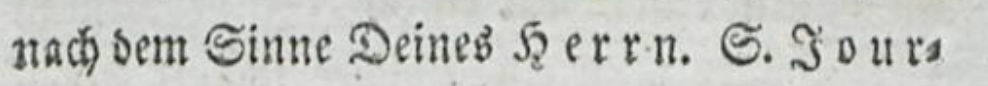

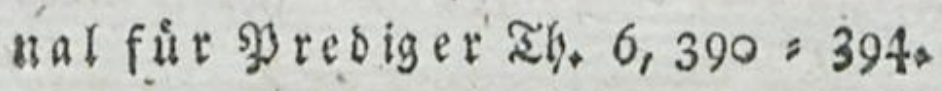

\section{2.}

Heber Den 1lmigang mit Betrubten.*)

(5) iff fojwerer, als man benfen follte, Sbetrúbte yon allen 9 trten wirelid ju berus rigen. Uub boch - wie oft wirb oer gुjez Diger zu Diefer Iु)flid)t aufgeforbert! Dems

*) S. গiepcrtoriut It $\mathfrak{5}$ 
โอ wits feine bemeitide zu finben fern, wo nid)t bięen ober jenem Subjorer mandjerley Leisen uno Beftummerniffe vorfielen. Stber wie oft fann fich ber unerfabrne গুoresiger vergeben, bajer troffet, wo er bod nidjt troffen foll. Sft greift or biefe Sache vera fegrt an. Es ift raḩr, ber gुrediger mus

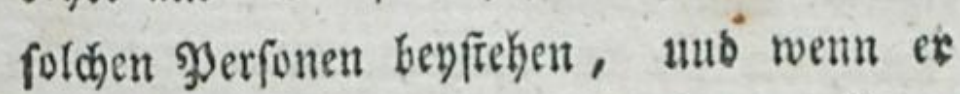
igre Serzen berubigt, fo erzeigt er ifinen

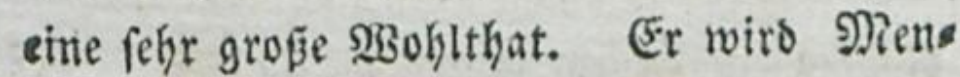
(c)enfreund und gleidfam ibr Erretter. Es sommt alfo \{lles; barauf an.

\section{I.}

Der presiger muf es genau uns terfuchen, ob diefe Zetrubnif uns Crauriglet etwo folge uno wirtung

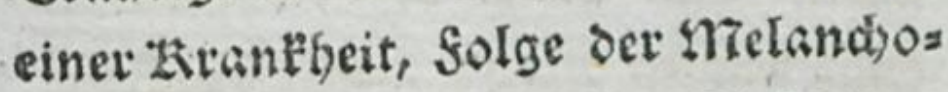
lie oder bypodsonorie fey. Diefes faum man febr leidit aus ber blaffen Farbe uno aus ben aufgefchwallnen 2rbern rehen. Sif Diefes sie Urfach ber Setrúbnif, fo mirs ber গुrebiger burd alle feine $\mathfrak{B} 0$ rffellungent

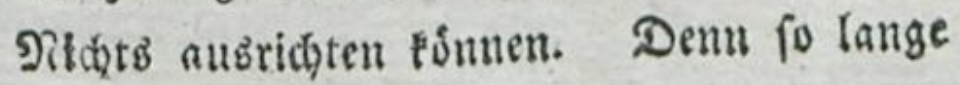
sie Frantheit nod nidt gehoben iff, fo lange bleibt 


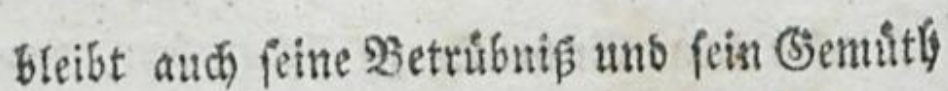
traurig uno unrubig. bier mufs ber 20 ; sas Beffte thun, die Siranfleit des feis bes heben, bie Safte verbeffern, die Stots fungen bes Geblitts aufbetien, ferkiges vers Sưmen, baß der Ltmlauf beffelben mieberum frewer werbe. Sobals fich sie Srantheit bebt, verliert fids aud bie Rraurigfeit uno

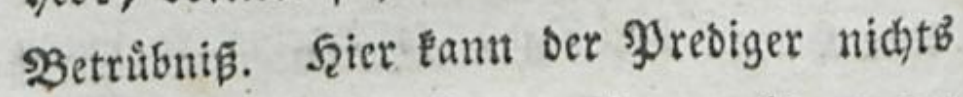
2nbers bey forden Reuten feyn, als anges nebmer uno zeitvertreibender Gefello fil)after, ser burch feine Erzäblungen dab (semuth Diefer Sranfen su erbeitern fucht, Der ifun feine Bebanfen you bem Begens ftande ableitet und fie anf etmas 2nders ridbtet. Mit (5inem $2 B$ orte: Joer fich alsె theilnekmender Freuno gegen ifn beweif't. f̧ier rebe iđ̆ aแb meiner vielfåltigen (5rfahs= rung. So lange wie ber $\mathfrak{A r}_{3} \mathrm{t}$ die Srants beit nidjt yob, fo lange fonnte ich an fols dyen Seuten शidits aubrichten, uno oft, wern biefe Sirantheit fo tief eingenurzelt roar, frarben fie in ber grósten হraurigleit 
uns ifre Fergen Fonnten nidgt Fernbigt wer= Dell.

2.

结 muf fich bemúben, Saf ex sen Reidenden uno Traurigen $g$ es n 6 u wendig. Denn nur wabre befelyrte Sinber Botteg find Des Siroftes fábig. Daker muf er genau unterfucben, ob ber Setrubte eirt befebrter ober nod sin unbelebrter ) ) (enf(í) (ey). Sift er ein unbefebrter, rober uno it Cunben dabin lebender श) (en(d), fo mus ef

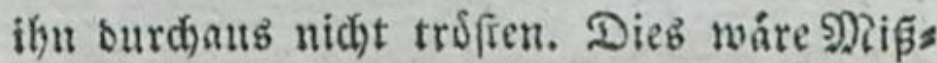
braud feineb Troftant.*) গein! er muß erft an ifm arbeiten, baper biefe ibm zus ges

*) 2lber - lieber Bruder - Dá bu auch for cise Reibende menfonenfreunbs lich behandeln, ons Du fie mit Riebe, Sanftmuth uns Sobomung allmăblicb auf fich felbfi jurücfifubren muß - Daß Du nicht wild mit bem Domer bes

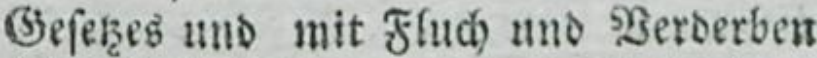
uber fie berfabren und Dadurd ifgr $\mathrm{S}_{2} \mathrm{~T}_{3}$ nuf immer gegen Dich verfdliesen, auf immer yon Dir abwenden Durfeff bas wirb Did) Deine Religion und Dein Sers ofue mein Erimuern lebren. 23. श) 


\section{4}

gefingten Seiben alb eine fehr frarle yaitertis d)e Ermahnung feines (5jotteg zu feiner $\mathfrak{b e s}$ Fefirung, Befferung und Errettung anwens be. Diefe jugefuigtp \&eiben fins oie frano bes 23aters, mit merder er ifn will ju fir zichen allb lauter Bsite, wie ihn aแb bem' Edjlaf der Sidgerbeit erruecten uno fein wabrez̉ Grlúă befórbern. $2 B e m n$ er on fulgt und ons Reiben biefer 3eit fo ans fiest uno betrad)tet; wenn er aufmerffam auf fein gefilbrteb \&eben wirb; uno fich entfhliegt, unter der Gnabe Gots teb̆ fich wabribaftig zu beffern: wenn er fols the untruggliche Siennzeiden bavon angibt, alsbann ift er erft beb Zruftes fäbig. Slbce

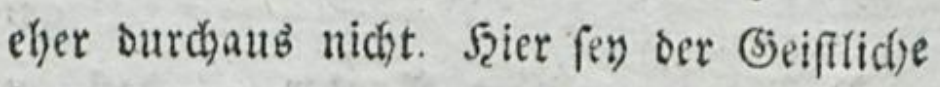
ia nicht ju voreilig, bandele nidgt unbegutfan, fonbern er reinige erff bie 2 Sunbe atts beur (brimbe beraub; bernad) tann er fie erft verbinben unb beilen, beilet er fie ju friths zeitig, fo fript ber Schaben unter fich uno wird oft unbeitbar.

Coictọe Seute fethen oft in bem gefábs liden Morurtbeile, als robite jeoes Lecis 
Sen siefer Zeit zeiden ser Fnade und des zeyfalls Gottes, ein fidtes niertmal des Gnadenfandes. 2tuch

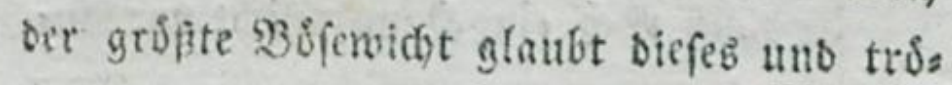

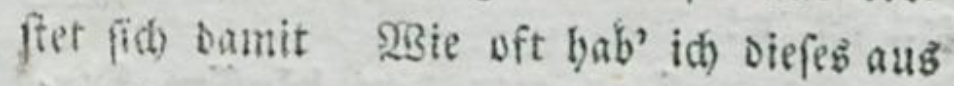
bem Munde offenbarer Sunber gefort : J J ficbe gewif redt bey Gott in Gnas: orn, weil er midh fo febr 3tidtiget.

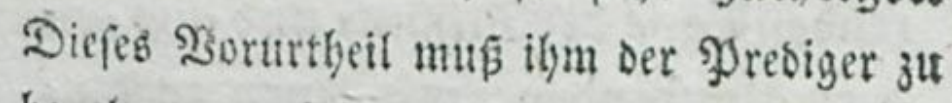
benelgmen und zu wiberlegen fuchen; er mus

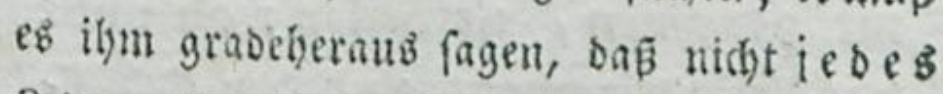
leiben ein semmzeidjen ber Bnabe biotteg (ei), fondern mur $b a b$, weldes (5ott wab)

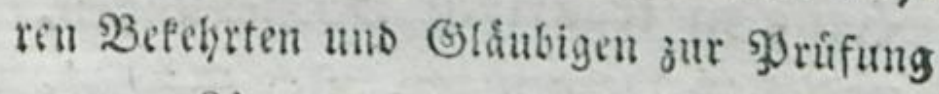
unb jur Råterung jufdjict. Daber merte fiid ber 3 rediger Die Scauptpflicht:

侻inige 3 trofien - Viele $3 u$ marnen, zu ermabnen uno in ein beilfames Edrecten $3 u$ fesen 2 tlle aber 3 untetridten, wie fie fidh in iben gegenwartigen $\mathrm{Lm}^{2}$

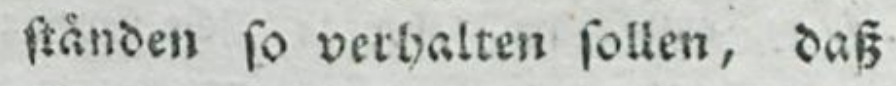
Gott, Der liebe uno der gutige Water, feinen Enojroct an ibnen 
erreide. - Darinn befteht sab Saupto

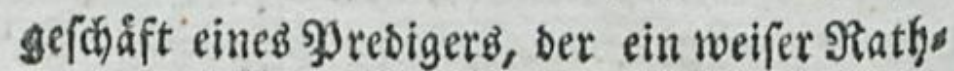
geber feyn will.

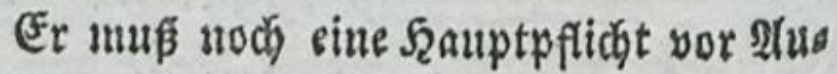
gen baben!

Dzr muf es aus ser blaffen fars be ses Gefichts, aus sem Ianfamen Gdilage des pulfes, aus sem fillei. fenden Cone ber Etimme und aus ans sern Umftinoen, mit jenen 3ufamo mengenomen, uttbeilen: $o b$ nidst sas Temperament und ser frênt. Iidue Juftans seo Kótpers, den bang 3u trautigen vorftellunger, und sas Dergnugen, fid Satrects siffe su extrciumen, verurfache.

Da mun Diefer fo oft gefdiebt, io sun ex siåtetifche vorfdaläge thu, unb iknen vernuำtige Gefellf daften, freunb. (chaftliḑen Umgang mit guten Nenfchen,

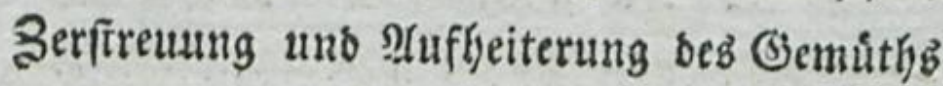
2. f. to. empfeblen, aber ia nidbt (3)otteg

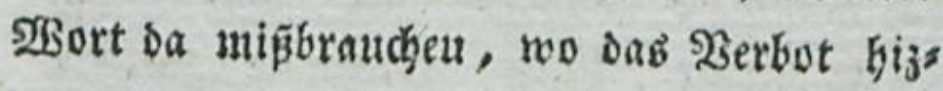

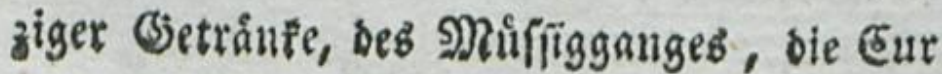




\section{7}

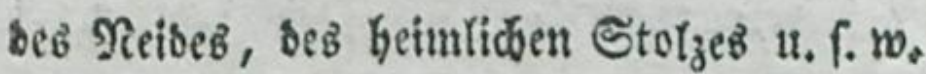
ain notbigften wåre. S. IIZOsbeims ฆroraI Th. 5. ธ. 448.

Interfudunge genau, wore ů ber siefe 23etrúbnif entitebt. Sie entfteht entweber liber åufferlide, Doer úber blop geiftide llebel, oder über beyse zugleid. Ilnter ienen verffeht man Durftigfeit, fdymerzhafte ober Iangwierige Sirantheiten, ungeredite Ses oridungen 2 nberer, Szerzeleio liber bie

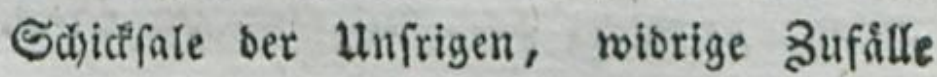
u. f. w. Geiftlicbe Leisen fino bie traus rigen (Empfinbungen über ben (d)limmen uno gefáhrlichen Buftand unfrer Seele, bie $\mathfrak{B}$ es angftigung wegen unfrer Sunden uno wes gen ihrer theils gegenwartigen theilb funfo tigen betrubten Folgen. Die Traurigfeit und setrubnif aber felbft ift entmeber eine un: vernúnftigeuno gegrúnbe te ober nus gegrủubete. - פ̧eruนnftig iff fie, wenn ber 3 ufall, ber uns nieber/chlágt, ein maks res, nidjt aber nur ein eingebilbetes uns er

2. 3 tråน 
traiuntes Hebet uns wenn fie sem Brabe beffelben fo angemeffen ift, das fie weber

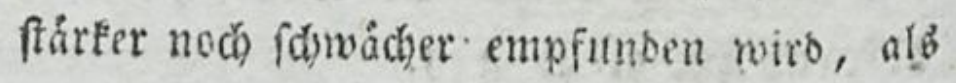
es unfer 3uftand erforbert, und wenn wir unb -nid)t forwobt un obs Hethels uno bes nåd)ften Binbruct'b millen, ben ç auf unb madbt, betriften, alb vidmelgr wegen bes fohtimmen (sinfluffer, ben eह auf unfre malys se şablfaht jef̧t uno funftig haben famm.

Es if wahr, ber sुorebiger if folten ium Stanbe, die nåchffe Urfach aufferticher Seiben ju heben. Er muß alfo mur babiut arbeiten, Onß er oie allzuleb bafte Dory fellung oavon fold w d be und vet: beffexe uno die Leisendon untet" weife, mie fie fich vertbalten muifien, Damit bie Dorfeloung an ibnen ien 3 weck exreide.

Wober fan er aber die mabre uno

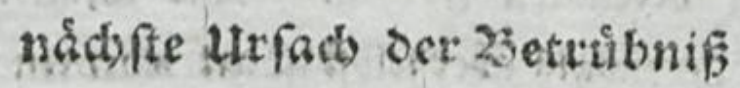
eqfativen?

Entweder you bem Betríbten felbft Dober yon 2rinbern. Daker mus er fíd gleid im 24ufang mit bem Betribten in eine fretulo: 
freunofdonftlidge und liebreidie llnterresung cinlaffen, mo fid) entweber yon ihm die Uliach feituer Befiummerniffe entbecten Inf: feu; ober ilym allerley Mrten yon traurigen Bufálen auf eine verfectite \$rt nemen, um aub feinem Mande babey zu ertennen, wels des baun fein Antiegen fin. Uno diejes thu' ev liebreitis uno rect treuno= (it)aftlid); im Tone deb theilnelgmenden Treunbes, bamit ber গुatient gegen ify aufrichtig werde, unb bafi or ifm alsbann

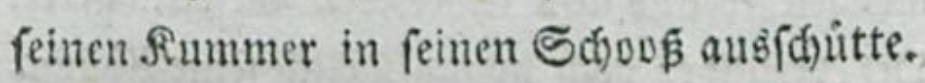

Merft or aber, onß̧ berfelbe die Ulrach feine Summers gern verbecît miffen wolle; fo fann or mit weiterm Padbforfden inne balten, uno er fann zufrieben feyn, wenn er nur úberbaupt die c faffe der Sirantheit recif.

Wie aber an wenn 2llles vor dem preoiger foll gefeim gebalten werben?

Si \{eh' the nicht ein, was er bey dem Sotienten nuşen fơne. Denn wie fann ber Arzt bie Rranfheit beyen, wenn fie 24 nicht 


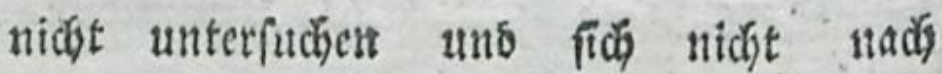
allen Umftanoen erfunbigen foll? - Dods bringe ber Syrebiger nid)t in ben Syatienten; benn fonfi migte er bas 3utrauen verlieren uno ibn nidjt gerne mebr fehen. श्tbbant ware ifm ser sugang zu feinem f̧erzen vers

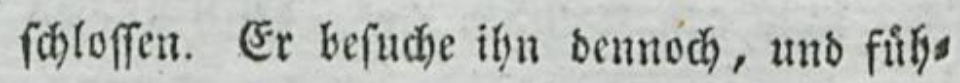
re einet alfgemein erbaulichen Dišcourb mit

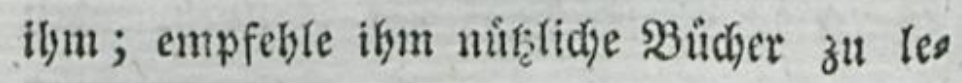
fen, uns zeige fich ing als einen theilnehs menben freunb und liebreidjen (stefellfarsafter. Sft erreidst er boch noch gluctich feine 2roficd.

4.

Der Presiger muf zuverIaffig wificn, ob die Zetrubuif von einem aufrodenden bofen Gewiffen, ober von einem 3 kirtlicben engen ober irti= gen Gewiffen Gerrulbre. DaB Erfte ents fpringt aus wirflich begegangenen groben Sinden, Laftern und Sergebungen; $D a \mathfrak{B}$ \&eģtere aber entftebt aus ber, \$orfeflung vermennter Sunden und von ber Einbilsung, als wenn uns getwiffe, aber febr unrichtige, Derfmale oes. Guadenfanbez man gelten.

รin 


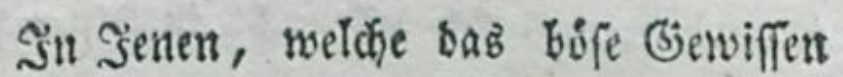
ángfriget, mú er einen feffr boben Eirno Der Traurigfeit burd) güttlide Scrwegungse grinbe hervorzubringen bemiket fenn ; einen

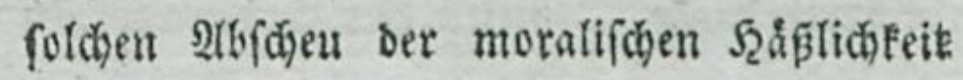
iffer Sinden, ber ftart genug fey, fie finfo

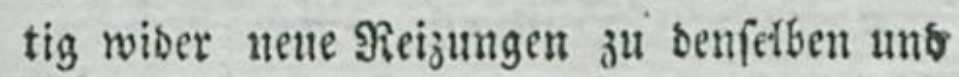
zu ifyrer beimlichen \$egebung zu verwahren. 2tber ex barf surchaus nidjt gleid) troffent. Diefes wåre fehr uniberlegt gehanbelt. ")

Rein!

*) Daв mirft auch bey fol cb en \&eibenbent nidt immer; oft wollen fie viel= melyr fich nidft gefdout roiffen, fie wolle n bab Sdirectlidje uno Eábimpfs lide ifrer \$ergehungen ganz libefehent tuto ganz empfinben, un fich ganz Der Berfnirichung zu überiaffen; eher bringt fein Troft in ihre Seele

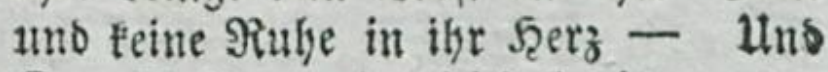
Der iff innen ein leidtinniger ober fdimacher, leibiger, umwirffamer Trós

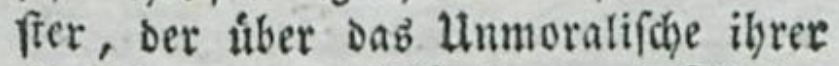
Szandlungen wegid)lupft, es mit Sdgein. grůnben entidultsigen, uno fie bariber feid)t berubigan will. Man büte fíd, savor. Solde Mienfoch miffenubers faupt mit viefer Silugbeit unb Mens

foreno 


\section{2}

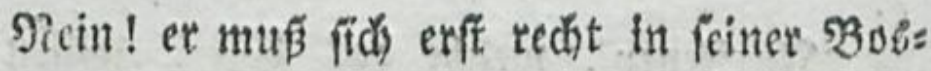
beit éennen uno die Siefalyr einfelyen, die er fiid) zugezogen bat, mit Einem 230 orte:

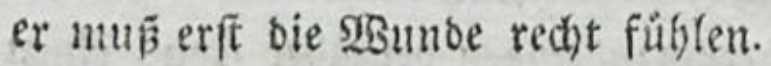

Doie aber - muf der prediger cin unffånelides Befenntnif ifer be= gangenen Ginsen ablotten?

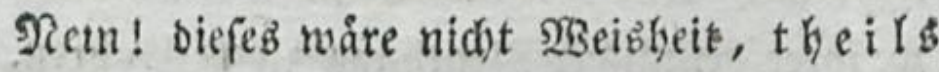
um nicht, wenn fie ber 2rffect wieber vers Iaffen bat, ifgr Sertrauen uno ifire liebe ou verlieren, uno theilb, um fie grabe nur mit oer innern moralifchen Şerabfcheu, sungomulrbigteit ihrer Seele ju befchäftigen, tbeil s audh, weil baburch die (Siemutber oft um oic Echambaftigfett gebrad)t wer Den.

\section{5.}

Ter previger laffe fid aber sud nidit outch ifse oft etfolgte 23erubi gung betrigen; benn eb ift oft nur ein fleis ner Etilffano. Die Traurigleit, die Gott wirtt

fdenfenntnis behanbelt merben, wenn man nicht ibr Butrauen verlieren, reis nen suect nidbe yerfehlen uno ganz yer, Eelgrt auf fie wirfen will.

23. 
wirft, beffert unt beilet bie Seele, aber fie tistet fie nicht; verleitet aud nidt zur Mes Iancholie; ift folglid) den SBufefertigen an

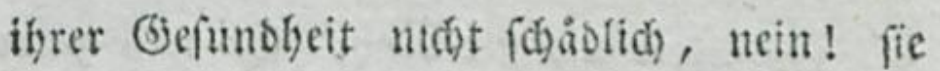
madbt fie alfmólich ber erbabenfen uno bautbrafteften Siube fahig. Daker bringe fie boch ia ber Syrebiger burd) feine voreilige 2fbpolution uno Rroffung nid)t um bie 2Boblo thaten einer langfamen und aub Dem Oruns be beilenben Cur. Er überlege bod biejes mohl: Sas sie Ziedtfertigung nux eine Gnabe fur bie fey, die in Cbrifto Jefu find. Siber feiner if mit $\mathbb{C b} \mathrm{r}$ : fo elyer vereinigt, alb bis cr lede Sinde allfo aufferfte baffet.

Die Berubigung erfolgt oft relyr langs fan; denn theilg bat Gott peine weifen

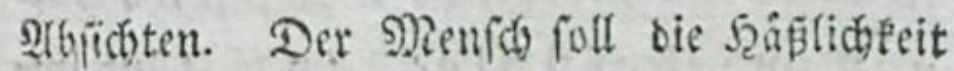

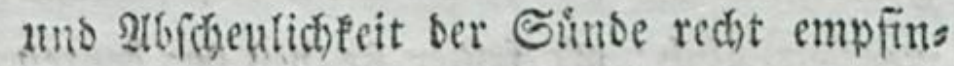
ben uno ibre Bitterfeit fdmeden, um fie yor funftigen Sinben vaterlich zu bewabs ren. Dft aber fectit nod eine gewiffe Sucle in ber Seele, bie ber Nenf(b) erft rectit eins feben uns verabfibeuen foll. Iheifs aber aud) liegt oft bie Schuld an bem 2 Rendiden felbit 
204

felbft, weil fich ser Syatient nod niạt red)t aufrichtig entfoliefst, alfen feinen ges beimen Sunden wabrhaftigzll entfagen. Das

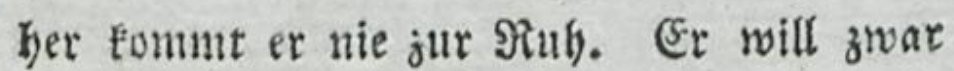
Der 2 ing gern los werben. Sft liegt ber Grutus in Deme inuern Sampfe iffer eigenen wibrigen Seidenfchaften. Soldhe fino feines Troftes fábig, bis oẩ fie fich aufridgtig untet bie J̧and (jottes bemútbigen.

6.

Der Prediger futtre fie auf bas einige richtige uno gerviffe Mieremal Des Jnadentandes: Die find in Shrifto S̈efu, bie nicht nach bem Fleifdje wanbeln, fonbern nadb bem (jeifte. (Rsm. 8, r.) 2lber

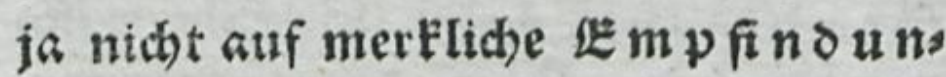

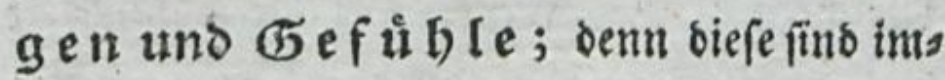
mer betruglid); oft fehlen fie bey ben befsten Gyriffen. Davon fosmen es viele naturlis dhe Utractben fenn. Mit Einem 230 orte! Er

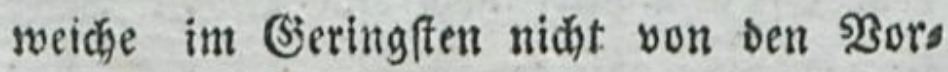
fóniften bes Shriftenthumb ab; verfalle nidate

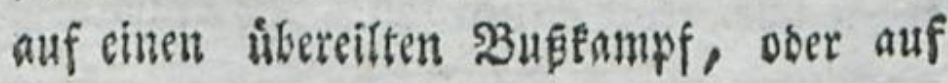

มุด 
פorffellungen, bie myfriod und $\mathrm{gan}_{3}$ fiuns lid) finb. Daburch verdirbt ex mebr, alb er must.

\section{7. \\ Der Prediger gebe genau auf ibs} re LEv3âblungen 2ldt, wenn fie ibm die Urfachen iber Zetrubnif entoes, fen. Er laffe feine \$ermunberung, ober cine vielbebeutenbe Nine felyen; Denn fon

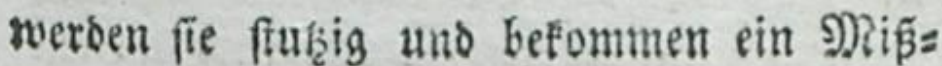
tratten gegen ifh, als wáre ifgm bic Sache 3u fdjwer. Sonbern er bơre fie gelaffen an; bemerke fid bie widtigften $\mathcal{S}_{2}$ auptumftanbe

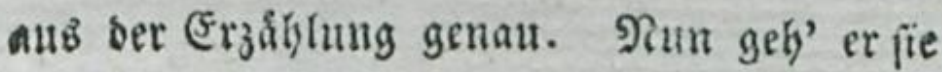
Stictweife burd), unb gebe ifnen auf jeden

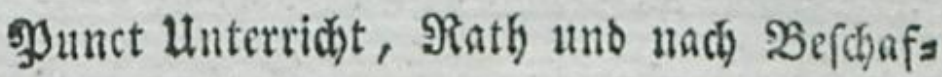
fenbeit aud Troft. शerfidire er fie nur,

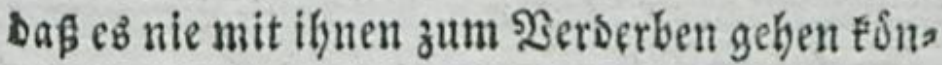
ne, Denn ein weifer (Jott unb liebreider $\mathfrak{S}_{\text {ater }}$ ber Nenfáen regiere alle Sdjidfale feiner Senficen, und lente fie endidi alle zuifrem

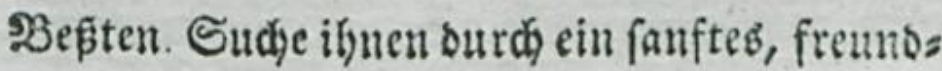
fonaftliches S3ureben einen guten Muth eimzufprecten. Den Unbefegrten gebe er stn Let: 
leitung, oafip fie befebrt, uno ben פeFely:s ten, das fie in ber Gnade Gottẹ uno in Dem Eifer Der Tugeno geftarft wetoen.

S.

Ev benuse ja jesen 2lugenblict, in weldyem einen feiner 3uborer fein, surds itgeno cine innerlid)e ober sup fevtiche Uifach in Z3ewcgung gejesteg uns erweidites ber3 offinet, mit al= Iev nur mogfid)fen Trewe uno ZXlug;: beit. - Da ift bas Jerz offen, um ben beşten Sanmen einzunefmen; Da ift er in bex

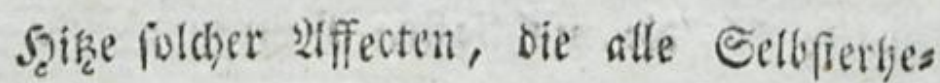
bung uno Gellffitåte nieberfolagen. Da fam er ein 2 Sort 3 แl feiner Beit mit gua tem erfolg reden. Benust er nber biefe. Slugenblife nid)t, fo fommen fic ify viels leidt nie wicoer. Miosheims moral ఇ\%. 9. S. $488=490$. Dem I ex s pres oiger bey oen 3 ettibten und 2unges fodtenen.

9.

vorzinglids aber gebe er genau 2lit, rab ste inverwanden, oder Sreuns 
Steunse, bser wothter suth the Linvorfidtigfeit nidht ras Gute bin= bern, das er befortern will. - Evls we Qeute triffen oft zur Llnzeit, bemn fie verfertyen es nicht; reben gleid vom lieben Rrenze, als bas গNerfmal ber Rinber Giots tes. Diefe bindern die Bnabe Buttes in ifren SBitentugen. Soer fie führen fie nod tiefer in sie \$Betrůbniß binein, frellen ibs

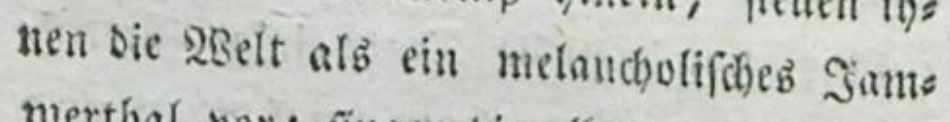
mertbal yor; fingen bie allertraurigften fies

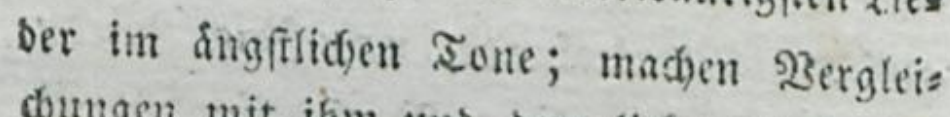
d) ungen mit ifjm uno bem lieben Seilante, n. f. w. Szier mus oer gy rebiger baralf febell, dafis fie verfandige gute tente befus Gen, uno das Die, bie um ifynen fint,

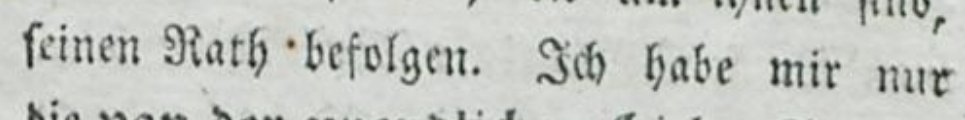
bie von ber unemolidsen Liebe Gottes, von der theurer Relofung Iefu (b)t = fit, aud oft LOb = uno Dantlieder ges wählt, nachoem es bie Hunftande forberten. 3. (5. bey unbefelyten empfabl id bab \&ieb: Jefu, der ou meine Geele :c. oder ein aliberb aub einem neuen Giefangbuch, 
bas feinem Buffand angemeffen wat. SBes Betefrtent bas fajonesied: Gollt id meinem Gott nidst fingen ac. oder Gey Lob uno LEby bem bodd)ften Gut $2 t$. Ind bamit fie ifnen Nichts aus ser foges nannten Sieuzlchule sorlepen surftem fo enepalyl id ibnen felbft bie Edariftert und zeichnete bie Setrachtungen, Dic fie ihy nen yorlefen $\mathrm{mu}$ pten.

\$sir baben in unfern Iagen einige fúrtteffs (idi)e ङdyiften. 2fnoadten im Leiden uno auf dem Gterbebette, von Iac. Friedr.

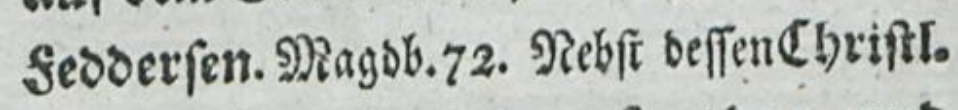
Dorfuriften bey sen freuben uno wiberwärtigzeiten siefes Lebens.

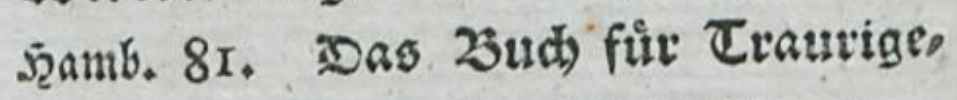

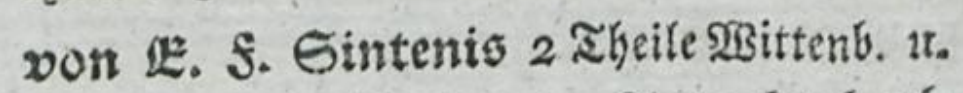
Zeroff. 8I. (2 thir. 8gr.) 2tnoad)tobuts fir Krante aus allen Gtånsen, vors J. G. St. Utrich). \&eipz. 79. (2 thlte $4 \mathrm{gr}$.$) G. 位. Walsau 2undadten firi$ Leibende, Zitante und Gterbende. 3 Th\% গরirnb. 79. ( 18 gr.) Sorziglich aber: Croftichriften 3ur 2fufridbtung fuit Leis: 
Retsense, sie tiber Sen Tod thres Gatten, Einser und Geliebten ttaute rein. Lin Suc für Samitien. Fralle 83. (10 gr.) Diefe Sajrift if fergr ju entpfeglett.

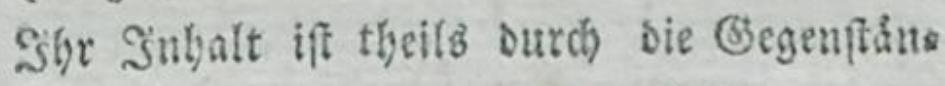
be uno verfófiedene Frten yon feiden, wore nuf fie fich bezieget, mannioffaltig - theils burch Den Ton, ber bals mefyr belegrento sund aufeltrend, bals mebt trófieno und zus redend if , vetichiebent. Wettentampfo Troftgrunde bey den traurigen Gabict: Falen ber menrath. - philotac. Ein Derfuch 3tw 3ertibigung uno 23eleba rung fur Leibenoe und freunbe dec Letoenden. 2 Th. Leip\}. 783 , u. 85 .

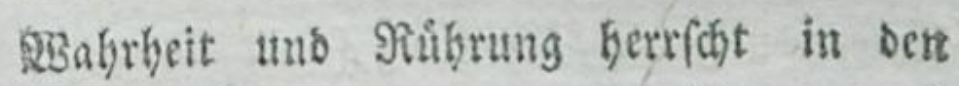
Troftgrinden bey den Gtibern unjs rer Gelicbten von Mi. Srieot. Traug. Wettengel. Gireiz 791. - Nod meht 2Birfung wúroen fie thum, went ber \$ortrag mebr abgefurta und weniger gefdumucf wåre. ")

Dิ

*) Dhne mein Letwas fint Criutende beym ToDe ibrer Liebent 5rans

D nuves 
Da biefe Eadie febe widjtig ift uno folyr biafig vorfonment, fo will id nod einto se Sragen aufwerfen und fie beantworten.

\section{I.}

2Bie grtwinnt Der Prebiget Dab Bus trauen eines fold)en Watienten, Det folyt betiubt ift?

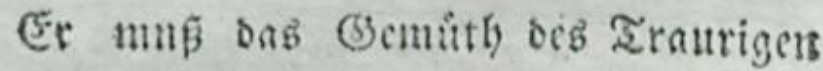
ourch eine indifferente (E) räblung uno Unterverung in bell Etand bes (sleid)gea

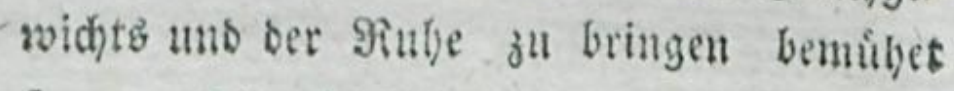
feyn. Şiernád)/ mus ex fid pein walya ses ober cingebildeteb \&eiben nufmerffom ers żdG fen laffen. Damu theilb surd) angenthme Alnterresungen unb Renfung feiner 2 tufmerta famécit auf angenebme Dbjecte z. ङ. feine Sugentiabre, Neifen, Finder u. ケ. w. bent Soetrifoten jerfitenen, theifo nber burd) bes zeis

nover 786 zu ben obenaugefüforten treffa lid)en Ed)riften zablen ju wollen, glalta be id boch ofye Unbefdeibenlyeit ben zite l befes meinez erfen fitternria fa)en פzerfucbs bier anfúbren zu bürfen, Da er von mebrern Siccenfenten febr gus Itg beurtbeilt uno sffentlid) empfoticu i] ${ }^{2}$. 
zeigtes aufrtątiges Noitteiben feine Siebe gegen ifun erwedert.

Der Wrebiger bente body niagt, als wenn ex mit folden perfonen ridots aโด Iauter Zieligonewabtheiten reden Dirrfe. Nein! er faun uns mus oft in sifferente Sadjen mit ifhnen reben, bie ifyr (Semuth) erbeitern und ibre Gebanfen yout Dem Leiben ablenten. Demn fino jène Htu terrebungen alle ermfthaft, fo gibt ex iffert

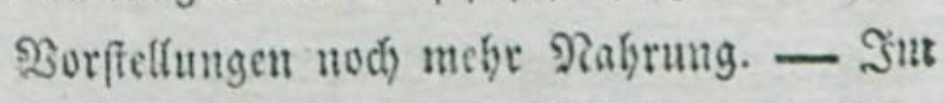

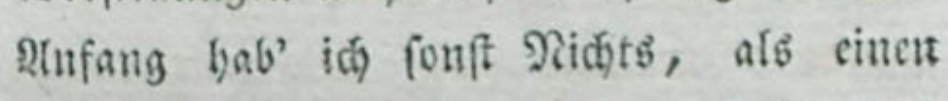
theiInelymenden Steuno vorgeffeilt, uns bybe inmer unter meine Utnterrebungen biea fe uno iene widtige Peligionstwabrheit mit

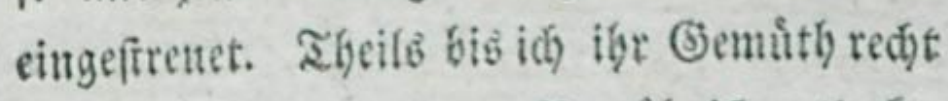
exforfdot, bie Atriad) oer \$etrúbnif entbecf́t,

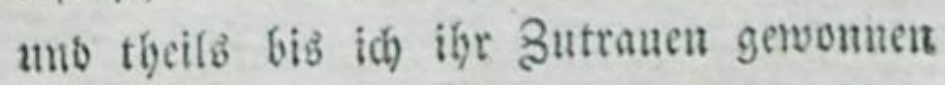

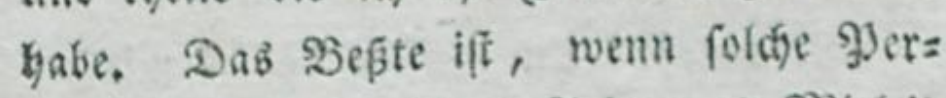
fonten an dem \$rresiger Riebe uno Nitteis erbliden, wem fie fethen, er bleibt felbit nicht ba obue (sefuht und Empfintuig, fon= Dern er if felbfi geriffet unt friblt igh \&eis 27 Dea 
wen mit. Deffo megre gerwinnent fie ifnt tieb, fehen ifn gern uns baben ątrauten zu igm.

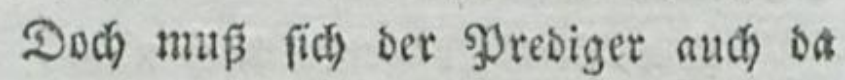
worfichtig verhalter. Sft formen folde şers punen aud) bie inbifferenteffen Sadjen nidge feiden, fondern fie wollen fonft Nidjtb, als geiftlide Sadben bơren, fonfi werben fie sers Drifstict. Da tiberzeug' er fie erft, Dás bie 2 Beft fein Sammerthat fey, uno Daf́ wir in ber 28 Belt nicht immer ernffhafte Eacjen reben miffen. Naein! wir misten aแd) bab Gemuith auf biefe uno jene erlaubs te 2 trt fuchen zu exheitern. Sleiben fie aber eigenfinnig - gut! fo hat er in ber \$i= bel fo viele vortreffliche (befthichten uno $\mathfrak{B}$ es

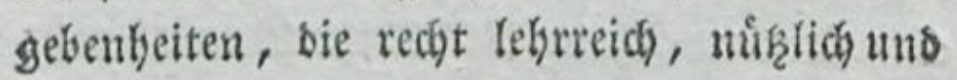

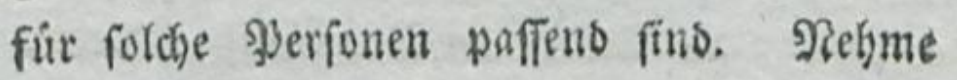
er biefe!

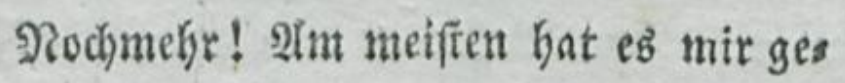
glicăt, wem ing foldie soerfonen auf die IEvadblung itytes Lebenslaufs gebradist Habe. Dft war er burchmebt mit fo man= stidfaltiger Sirte Botteb und feiner befons Dern 
betn Borfefyng. 2luf biefe madite id fie aufmerffam. Da bab' id oft mit ifnen viele Stunden gereset. Szernach erzaghte id itgnen eine Gefojiçte eineb feibenben, bie fehr merfwurbig war, u. f. w. Daburd zog idh ifyre Stebe auf mid̄, uno fie battent gegen mid Butrauen. Nit Einem 2Bort: Der weife Presiger nuß 3 ufelen, was bier an oienlidiften ift. Er muf felleft einen guten \$eobachtungsgeift baben, uno

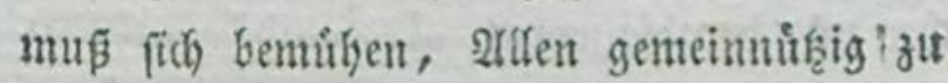
suerben. Se fiebreicher, is fanfter, ie freuntos fogaftlidger er mit folden Reuten ungeht, deftomelyr gewinnen fie ifgut lieb und babent

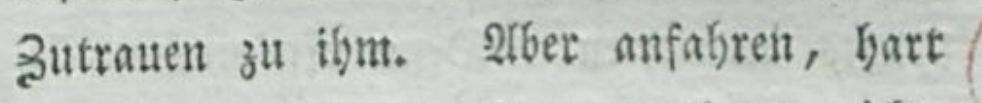
mit ifynen reben, barf er surdjaub nicht,

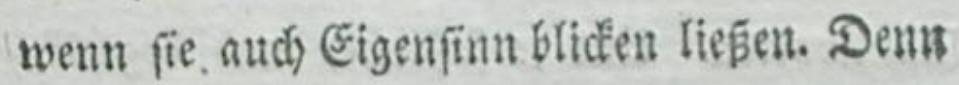
fonft verliert er igre \&iebe gleidy.

\section{II.}

2rie muf et feine ltntertebungent sinvid)ten?

Uteberbaupt muffen fie alle Iidjtwoll, seutlich, fâflich uno wabr feyn. Itber

D 3 fie 


\section{4}

fie muffen befonbers bie frorm nno bie Stâts fe ciner cated)etifden Inouction baben, fo, oaß ber Betrúbte die Berubigungsgrims De auch in ber 2tbmerentyeit bes গুJrebigers

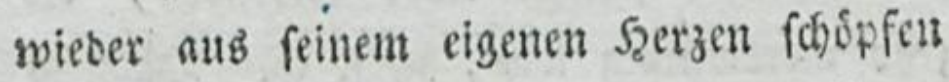
und fie fids felbft wieber vorgalten fonne.

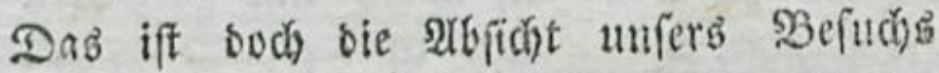
uns unfrer Unterrebung mit folden गुerfo: nen, unb ba verfeblen wir nie bes rechten Swecti. Je licbrvoller, ie beutlid)er, faflis d)er uno berablaffenoer, aber nud je ríbrens ber bier unfer Sortrag iff, beftumebr gea fillt ex foldten Derionen. Şat ber DुJebiger

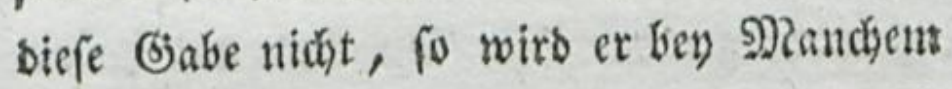
গididţ ausridten. Denn oft machen fie 3ૈweifel. Der झुrebiger muß fie glei (t) beantworten. Ino je faflidjer uno begreif, licher sie 2lntwort ifĩ, befto leichter uno faflidber verfebu fie felbige. Slop patbes

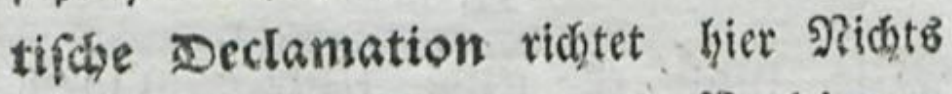
aus. Lange Zieben und Preoigten fino vergebens, Sie ermuben ben Bsift ber Reibenden unb fie werben verbriflia). Sie ponmen aber auch aแb fold)em weitläuftiges

Lin 
Itnterrialt gar Nichts faffen; ber fluge uns woriditige 3ुjediger met oft abbrechen, uno ben Letoenden reben laffen; ilym wicber

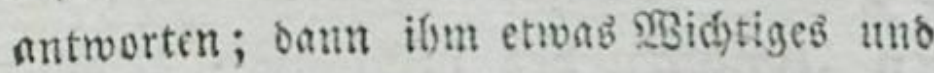
auf feinen elenden Buffand (iid) Syaffentes erzählen. Uno alfo immer bie Seele ber Seibenoen in Nhfmerfameet ju crbalten fus chen. Dft aber wiro dab Beten mit folo

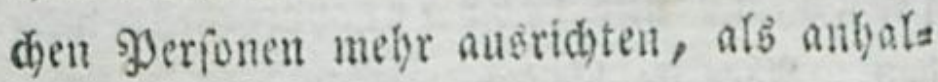
tendeb Bureven. Selbfi der Mgrebiger muF barauf fetgen, weldhe $\mathfrak{A}$ it bey sicfer voerbey jener भुerfon bie fobidflidfice ift. Daber muß er 2tltes zu erforidjen fuden, bamit er fich nicht felbft fein 2lmt erfichere. Denn sent einen झुatienten gefállt biefés, bem 21noern

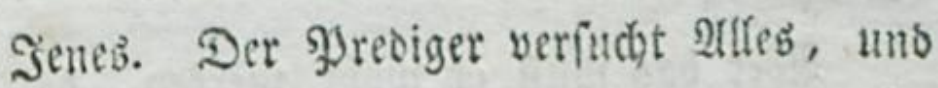
alboam bandeft er nack ber beften und bea quemften 2(rt.

\section{III.}

DrBie miffen feine Sroftgrimbe befd)afs fen (el)n?

Sie find theils allgemeine, theils befondere. Zu jenen gehoren die War fellungen von (5ott, feinendeigenI

14 ten 
216

ten uns feiner Dorfebung; won bee $23 e$ faffenbeit und $23 e$ ftimmung ses gegenwårtigen Lebeno; von den weis fen abfidten bes beftandigen wed

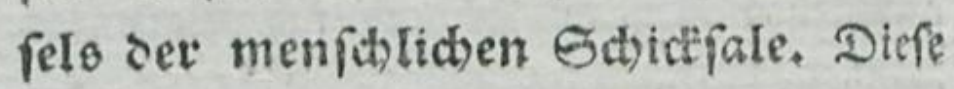
muifen foldoen झlerfonen mit ber materifdyen Befdidflidfeit eines Geneca fehr an= (d) a uend uhe rebbaft vorgeffellt wers sen. 2tber fie bleiben bod immer nur शies

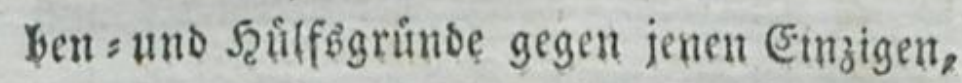
Der in ber bofinung jener fuinftigen Geligfeit für alle Sinder Botteb liegt.

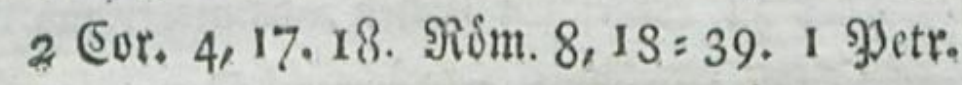
I, 6. 5, I0, 5̧ebr, II, I, 25. I2, I, 2. 7. II. 22 .

2tber bав mus ex woht merfen, Daf Siefer lef̧te Troftgrund nur bey $w$ hrbafs tig Gläubigen muffe gebraudds werben; senn Deren J̧offnung bes erwigen Lebens if allem gegrunbet und $\mathfrak{2 B a b r b e i t}$. 2(noerk nun er's aber fagen: baf Fott aud gegen susgeartete tiinder guitige uno beilfame Gefinnungen bege. 2hp. Befd\%. I7. 
77, 27. Bepunsers sas 3eyfpiel ded verlobmen Sobnes fuc. I5.

Die befondern Trofigrimbe miffen Had ben einzelnen 2trten ser Leioen, lo

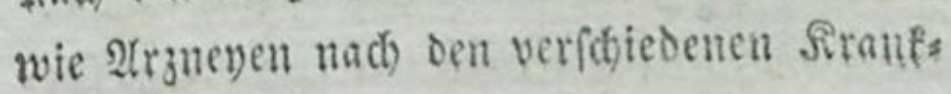
beiten, eingeriátet werben. - IV.

OrBic aber - 23 emin folcte fcibende fertr aufgebradis auf (D) fino, bie th), nen biefer foriden rius thoorfichtigfent, QDer aus Seinofeligecit perurfacb)t baben?

Diefe muf ber भुrebiger gaแ⿰ yon beณ श्attefurfact)en ab, und bingegtn allein arf

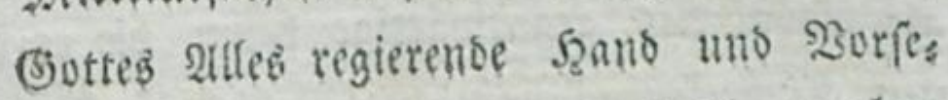
fung bintenfen, baf fie es einfeben, obne Gotteb weifer Regierung Psnnte ifnen aud bas (jeringfe und STeinfe nic)t wieberfahren.

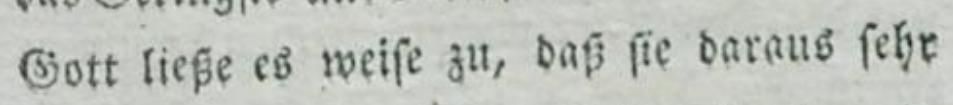
piet Butes fernen follen. Soh. 18, II, 19, 11, Jac. I, 2. अf. 77 , II. II6, I3. S. p. Mrillers Lebrbuch ser nTotal 1. $16 \mathrm{I}$.
25
V. 


\section{V.}

2Bie Eam Der Dresiger am fiet)erfen Das Sgers cines \&etoenden berthinen?

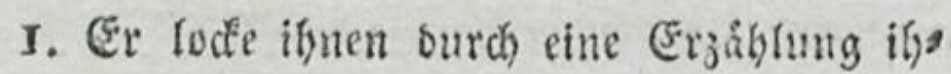
rer \&ebenegefdichte felbft das freywillige (jes

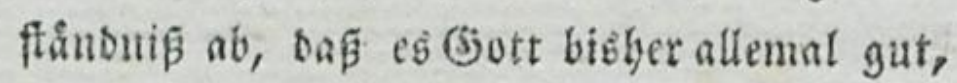
und wie $\mathrm{c} b$ bie (5ur ihrer jebesmaligen (je)= multhsart erforbert batte, mit ibnen babe machen woffen, uno laffe fie felffit bieraub sie Folge berteiten: Gljo muifien aud biefe der Sinnlichteit fo bittre Suth, rung oie weiffite und vaterlidbfe feyn; wenigfens in Berbinbung mit ber fluftis gen, unabfeblidgen Dauer jenez̉ \&ebena, atz wovon bab gegentwartige faum bie er: ften Sinderjafye nusmadte. (Sott wirbe auch) aแb Diefer Finfernif Daz \&icht bervor, rufen, uno ein fecheinbares liebel zu foinem uno Des \$ु ublicum wabren Beften anjuments

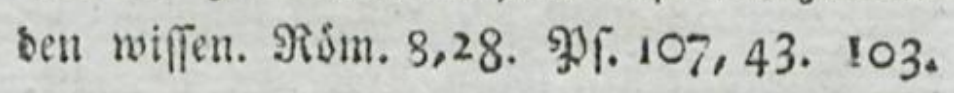
2. 3. 4 . 39, I I.

2. (3) th richte jebes Seiben unfern geifflid)en Deourrfnifen und benen von \$gm ertheilten Sir\&f 
Siriften gemíf eirt. I Cor. IO, I3. 2 Cor. 12, 9. भुर. 73,23 .

3. Sie Iernten Gott, die Religion, fičs, ber Huterfajico wabrer uno verganglidact Giter gamz anbers, als vorber, erfenneu. Hno biefes ift febr nüşlid uno beilfam.

4. Sie beţamen Durch Die llebung feftere Eimfichten und nene Sidfte. Sióm. 5,4. 2 Eor. $4,16$.

5. Sie lernten Gerglid uno inbrinftig beten, uns gewoignten fich an ben freten Hungang mit (3)tt. গु०ศ. I8, 7 .

6. Jefus unb fein SBerbienft unb bie fich barauf allein grunbende Errettung wirbe ifnen immer theurer unb unf disbarer; forg.

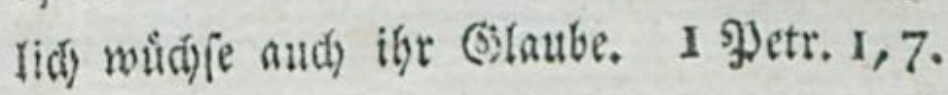
4, I2. I4. 12 Eor. $1,3=5$.

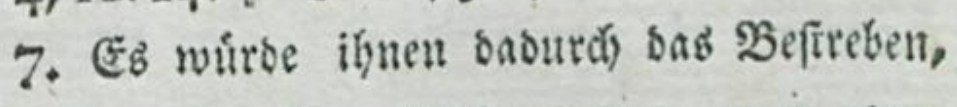
inmer Iefu gleidgefinnter ju werben, surch bie aufferliche (Bleiôformigfeit mit

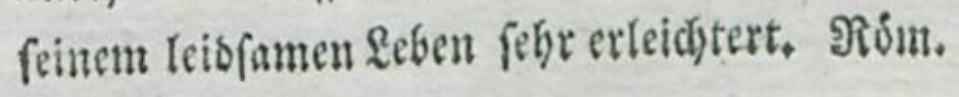
$8,9.29$.

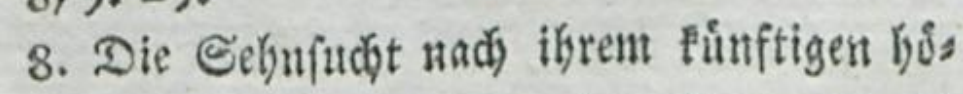
bern und beffern Reben wirde inmer feuris 
ger und thatiger. 2 อor. 5, 24. Róm. 8, 22. 23.

9. Heberbaupt liefen fid) viele Tugenten ofy, ne Leiben gar nicht aubiben, uno gerwiffe grofe chriftiche, febr erbabene (jefinmuns gen obne fie folechterdings nidit lernen oder amehmen. S. Millerg Znleit. 3ur weis fen u. gervifientyaft. Derwalt. Seg evangel. Leliramts. 6. 92. S. 169, I 7 . Riofermulterg 2InIeitung firt ans gebente Geiftiobe. 5. 54. 5. 66.67. Jacobi's 23eytrag sur paftoraltheos logie ז\%. I. ธ. $218=240$.

\section{3. \\ Heber Die befte und nisfictiffe Ant 3u itipfen.*)}

\section{r.}

Der, weldher Letoenoe tróften will, foll nidst blofs sie 2 bfficht baben, fie 3 berubigen, fondern auch, fie

*) S. Repertorium 4r ฉ็. 
fie 3 beffern. Sুiete भुrediget geben fid zwar Mille, biefe \&eibenbe zu berubigen; aber Das laffen fie gewsignlid folden Elent

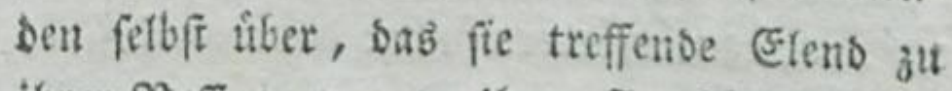
ifser Seffertung, ju ifyren Fortfigritten in alfen driffidident altgenben, zur mely= rern Rebensigmadyung ber beiligen uno götts lichiten Empfindungen, ju benen bie Reelts giou erwecte anzuwenten. IBsenn wir ifs

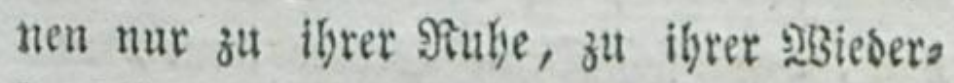

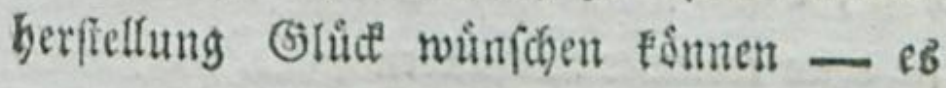
auch) zu ihrem $\mathfrak{R}$ us 2Siberwårtigfeit gehabt baben, thun, 28 em

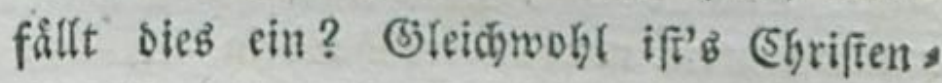
iff's Menf(d)enpfidid, fich fortzulbelfen, fich weiter zu bringen, fid volffommer zumas den, fich anzufaffen uno bem erbabenen aiét

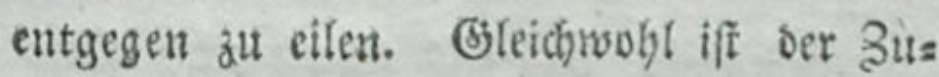
ftano ber Reibenbett recht eigentlid) bazu ges macht, zuzunefmen im Guten. $2 B e r$ diefe

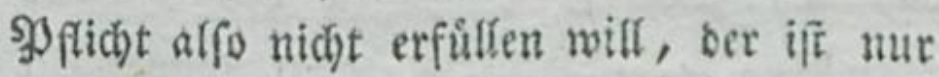
balb jum besten lumgang mit Betrúbten aufgelegt. Sein Troft ift mur Malliatio? (Eur, nicht aber f̧ebung deb Hebels. Der

leis 
Leibende mus es nach uns nach einfelyen, baif

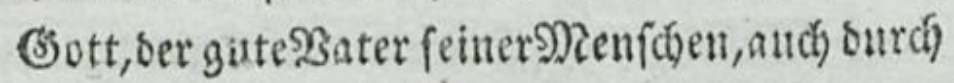

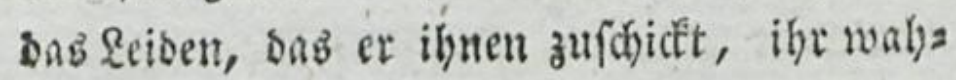

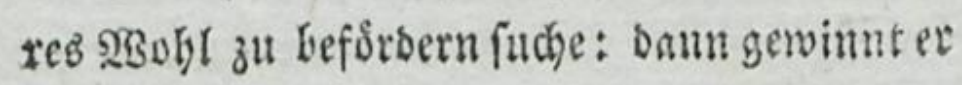
siefen guten $\mathfrak{B a t e e r}$ lieb, unto er ringt nad) feinem 2 enfall uno nach feiner Liebe. So frebt er nady Sofleommentyeit, uno Das burch fommt er bem Biele inmer nåber. -

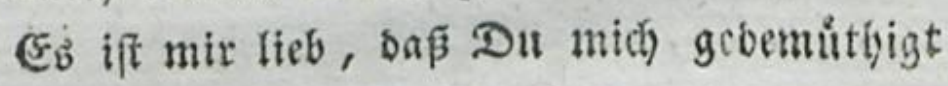
bafr, Damit id Deine Gebote lerne, uno fie befto reslicher aubzuiben fuche. Sprache Davios - Spradie ser Erfafrung. Gott fan al anberz beneen, nicht anders Ganbefn. Fremin! weiche nidjt aub, Damit Gott an Dir biefe gute 2lbficht erreidye !

2.

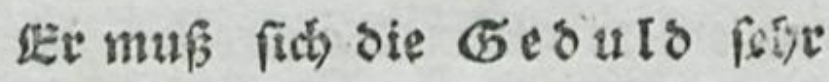
emproblen feyn Iofien. Es ift fefre leidst,

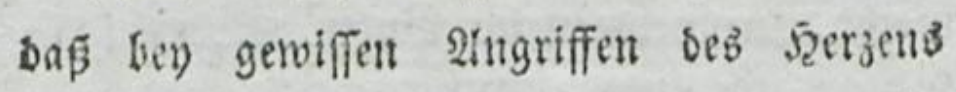
felbft Die, yon senen wir fonft immer cint fich gleidjbleibentebs, rubigeb şerbalten ges wohnt waren, anfangen, mit ber SBelt uns zufriebener zu. werben, unb fict) mandiestus= Drince 
sride im 2!ffect 3u erlauben, sie fie bey einer beitern Foffung ibrer Gecie fid nid) yerzeifen wurben. Eben fo leiăt if es auch, Das Der, ber mit ifnen umgebt, babures

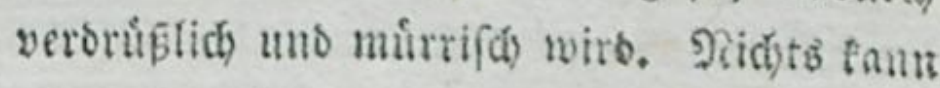
aber nieberfálagenter fúr einen betrúbten fern. Er madt fid felbft Sorwurfe, une sugleid) frånft ign bie Unfilligfeit, mit ber man ifjm begegnet. - Eolt idj cinem \&eis senden nod mefyr feibrn madien, ba id ifm soch gern Erteichterung füjaffen wollte? Nein! (c) will miđ̆ bemúben, immer mit Siube, mit Sanftmuth uno berjlicher siebe 3u ibm zu reben, meine Gebuto foll unermis set feyn. Sich will es mir felbft prebigen,

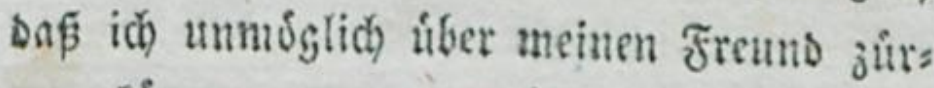
nen forne, wenn er liber feinen Siorper

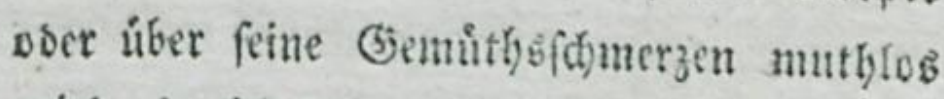
swirb, ba id felfer, der id gefuns bin, in

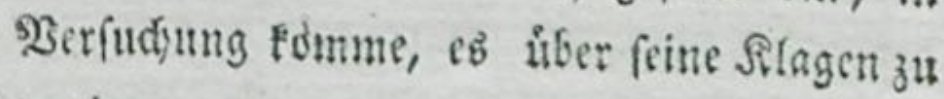
twerben.

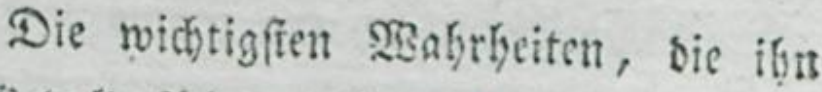
zur Gebuld futhren, liegen in ber gropen,

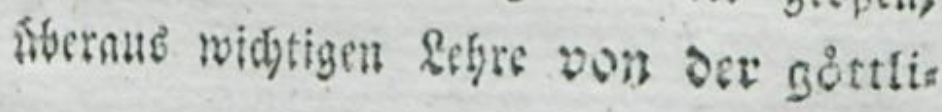
6):3 
then Dorfehung uno weifen Regtertng. 2Ber liber biefes Enpittel nidjt berest fenth Fann, ber iftzun Irofter verborben. IBse leid) wersen bricfenbe $\mathfrak{L} a$ fren Dem, ben man'в in Der ganzen Stärfe faun empfinben laffent eo ift cine Dorfebung! (Sott, bas befte,

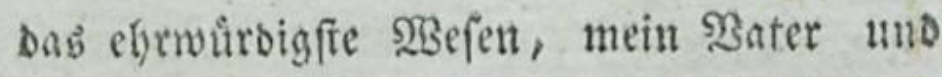
Srerr, feunt mid - faunte mid, ef' id wat, fall alle Bage, eb' fie waren, Jafhlte jebe Stunde; wog mein Reiden und mein (j) hid: Da if teine Thråne, bie ba flefipt, welde Er nicht falle! ba if feint Senfaer, ber flagt, ben Ex nicht vernábme. SBBie follt' (Er mid) aแb feiner 2tufficht laffen, obne beffen sisilfen bet sogel nidjt yom Dade fillt? Sin td) nidht melye als ber \$ogel?

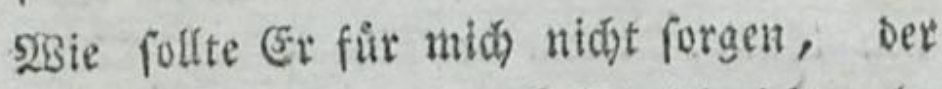
firt bie (stabblumen forgt? SBin id) nidft mehr als fie? SBie follte Er mid nidjt abten, Der mir alle meine Şanre auf bem Seaupte

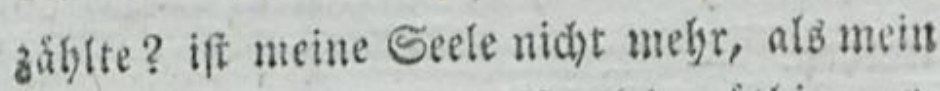
5zaar? IBie fosmt' idy alfo fleinmutbis wats Den uno zagen? 


\section{3}

3.

Let muf gleich im 2lnfang oghitis arbeiten, daf fie flbren boillen dem gus ten uno beiligen Willen Бottes finds lid geboriam unterwerfert. Diefe Interwerfung if ein Suftand ber $\Re$ tube, int welchem man mit Alleent, wab einem begegs net, zufrieben iff, - ber nidit, aus

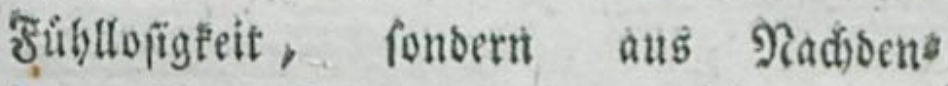

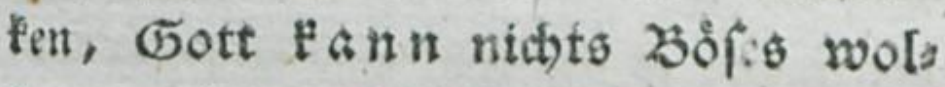
Ien, entffeft. (Er bebt dic Reiben nicht auf, âber bie 2 nngft, Das Empóren gegen (Bott, Die und)riflid) Unrube ift niebergefdlagen, bie Stútme ber \&eibenf(haften, unter benen oie Itnzufriebentheit gewis keine ber geringfen ifto fäweigen: Der, weldher fidh nicht ibe unters werfen will, fondern wiber bie góttlidge Şorfehung murrt, fommt mir wie ein Rratts fer yor, ber in ber Jieberbişe muttet, jebeß

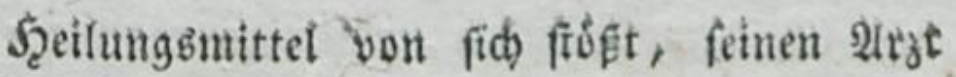
ba fat, oie f̧anb, bie ibn beilent will, yort

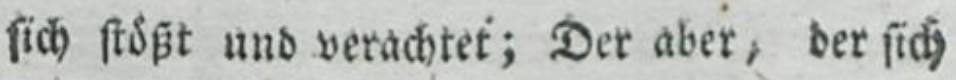
unterwirft, ift wie ein Sermunbeter, at bem tein Brited whe Sdumerzen ift, bet abet volltig zufrieden sarúber feine 2 Bumbent 
Ginreid)t, nud bie beilfarften Shittel ans

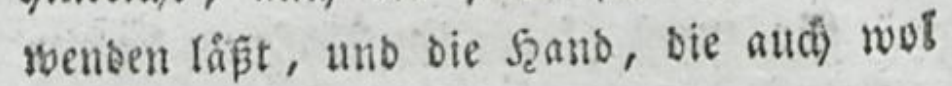
(b)lieber abfducibet, fuffen fann.

Wie bringt man aber diefe Unterwers. fung bervor?

शBenn ber গुgrebiger gleid) im 2lnfange, elye bie \&eiben nuf einen holyen Grab feigett, ธab Şerz von ber gropen Edhnáche unferer (Einfic)t, zumal in bie gittlidhen NBege, 34 liberzeugen, eร ifsm recht anfdhauenb zuma

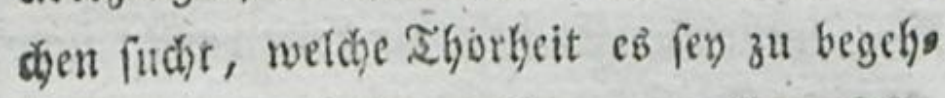
ren, uberall ficht feben zu wollen. Diea eร faun man befonbers aแ bem 23 แd b to b (weld)es ufberbaupt in biefer গiudeffid)t ein fegr merfmutroigeb Etide des sllten Zeftas mentb if ) abfernen, wo (sott eben bab SRittel braucht, um ben פRenichen uns terwerfung zu lebren - Eben fo mus

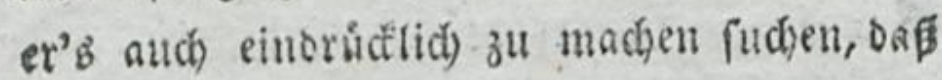
woir bey bem 2Benigen, wab wir aแb berss ficher bringender \&iebe zu ifm, aแb innigem Dant für feine zu uns tragende \$zatergüte, thun, ihm fein befferb Dxfer bringen tón. nen, als renigfens um feinetwillen aus Uแต 
Gatertwerfung, aub (Befiorfatm gegen ifgt feiben, und bab 2lufgelegte tragen. Denn er hat eş ia nie bơfe mit uns ges meent, und uns immer fo gut und fo fanfe gefulfyt: ro for es bod wol cinmal unfre 3ylicht, ibm fitll zujufitgen, ifm geoulbig nadjufolgen, nut feine \&eitungen nicht zut sergeffen, nur nicht von ihm jumeichen, und Brumnen beb Trofteb zu fuchen, die fein SEaffer traben.

4.

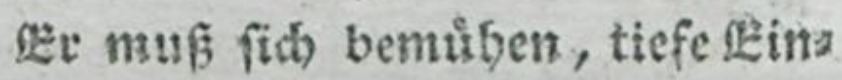
Sriocse in oas Gemulth oer Leioenoens volt $8 \mathrm{~cm}$ ₹ $4 \mathrm{at} \mathrm{e}$ in feiner Leiden 3 at

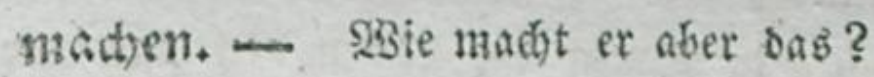

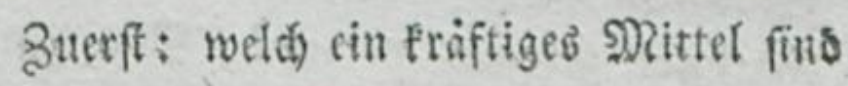
oie Seiben, unfer Gemith ju einer gewifa fen ifum fo anferorsentlid heilfamen Stille,

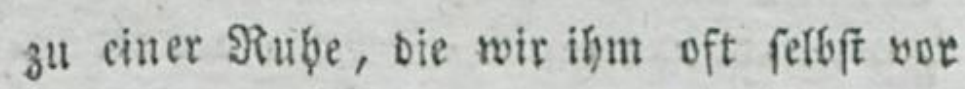
fagen, zu bringen! - $30 n$ biefem Bea Danfen whirbe idf) befonderb in ben ltmgang mit Eolden (bebrand maden, de yon dem grofipa

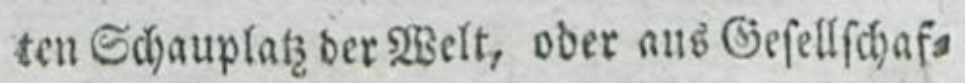

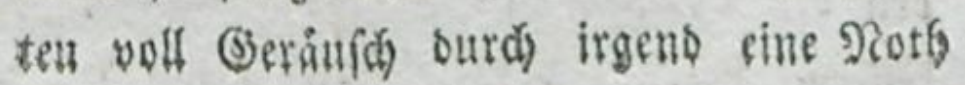
2 . bers 


\section{8}

Eetattgeriffen, fich in bie Einfameet atip eimmal you ben ihnen zur Gemblybeit ges worbenen Berftreutugen bes (Bemiths vers Iaffen fehn. Da befummt bas verirrte Szerz Beit nadjubenten, ba wiro ber taumelinoe

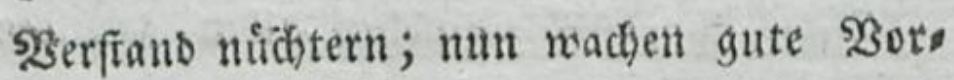

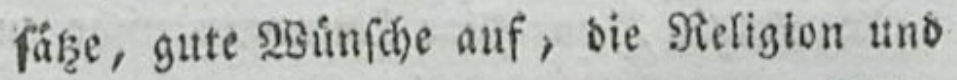
Tugeno hat Feoffunng ibren verirten Eohn wieber in igre mutterlident Afrme cilen ju feryn. - Eelige Tage ber Reiben, wenk fie biefe Ablid)t erreldyen!

F⿻ernach - SBeldie Feiben yon Tus genten, zu benent wir in ben Tagen bes Giluct's gar Écinen ober wenig 2tnlä haben, fosnnen it Ragen ser feiben vou uns jut Fêrtigfeit gebracht werben! Man entwerfe

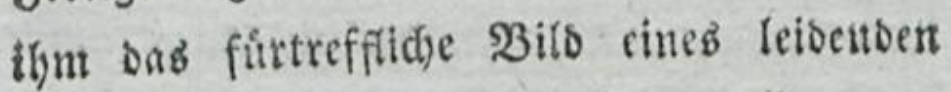
(Egrifiten, uno burch)webe diefe Betrad)tungen thit $\mathfrak{B}$ eyfpiefen aus ber $\mathfrak{B}$ ibel, cinco $2 \mathfrak{l b r a s}$ bams, Jofephe, biobo, Davios, paulus. -

Ferner zeige man ibnent wie bie \&eis ben ber SGriffen fie ber Şoffinung eines beffern debeng vergewifferm pśnuen. - 
Sin frot, baß Du Seibenber Deinen fofn ni申t bahin baft, fondern dafi Du ihn nod erruarteft! Sey froh, , Das Du einen (Jrumb mely baft, Deine Gemighbeit ienes Lebent zu boffen, ber Denen mangelt, weldhe in biefem \&eben nie geweint, uno bier fobon Iauter (5)utes die Fülle genoffen baben.

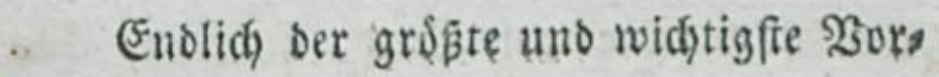
theil aub ben \&eiben biefes Rebeng ift bie Iebenbige Erfahrung ber Straft unb Deß

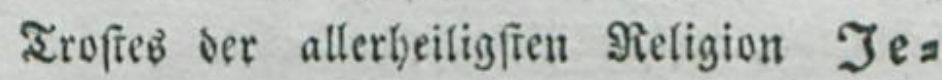

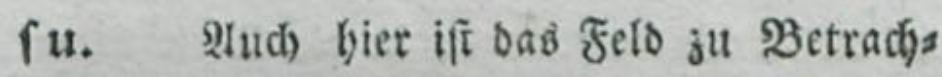
tungen unermeflich. SBBeld)e Ebre, ju leis ben, um (b)rifto agnlich zu werben! -

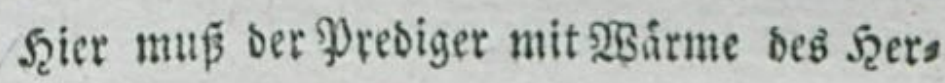
zenb yon biefer Sagahrbeit reben, unb fidh Des grofen Evangeliumb yon Efrifto nid)t fohamen; oenn feine Bebanten find fo felyr fúr Reibenbe gemadjt, alb̈ eben biefe. 2 Benn Der \$resiger oaber ben Betrübten feinen (s.s lifer yor bie Augen malen, ibn befonders in feinen Reiben ihnen vorftellen, wenn er alle feine liebe, diefés grofe Thema unfrer sobgefänge auf Erben, unb cinft im Ş Simmel,

$$
\text { भร } 3 \text { oft }
$$




\section{0}

oft warm empfinben, uno biefe warme Eras

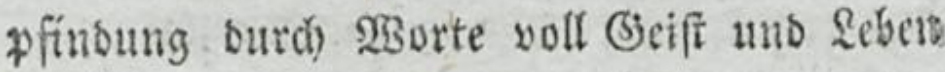

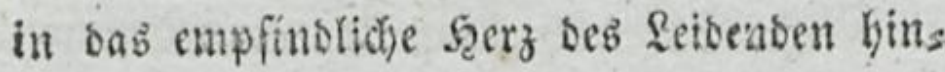
ifterftromen - wenn ev ibn in feiner 2 lngfts. in feiner Setrúbnip bis zum నobe, in feis ser alle \$ermunft úberfteigenden S) Jenfchenlies be, in feiner fómerzenswollen Duaal, its feiner ßerlaffung vou Cbott, in feiner నos Debangft bem 24uge bes boffenden (3) lnubent: abmaten esmite - 0 ! welcher Segen, wels d)e Rube, weldbe bimmlifbe Zroffung wirb. ans Siefen $\mathfrak{B e t r a d j t u n g e n ~ i n ~ b i e ~ S e e l e ~ f o n t ~}$ men, wie wirs fie erquidt, gefteret, wie awo bem Staube empor gehoben merben!

$$
5 .
$$

Les gibt aud gemiñe Leiden, vor senen in diefem Leben Feine LevIôfung ईu bofien ift: Gold)e Leibende muf. ef auf die 23 etrad)tung eines Pinftia gen befiern Lebeng firbren. Hebers Gaupt bleibt es gewipa, baßs in teinem 3ns fanbe unfers Lebens ber Gebanlff an eine felige Unfterblidb Feit fordje liberf(b)wangs liche Berubigung fogenfen fam, alo zur. Seit 
Beit ber Seiben. Sate bas bitre Erotreida yon ber feiţe verbrannt ben milden Regen, wie bie Blume im Norgenthau ben freunbs lichen Somenffrabl begieriger trinft: fo burdjoringt ber fajpe Trof ber Ervigfeit wie ein lieblicher Salfam befo erquidfenter bie

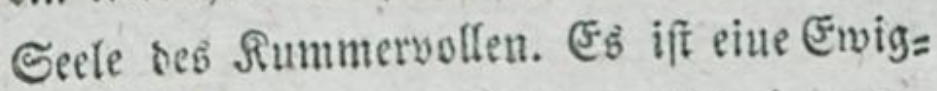

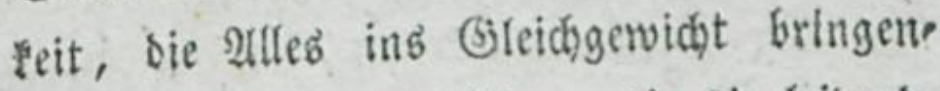
bie alle żweifel enthúllen, bie bie leibente Tugeno fdablos balten, bie bas triumphis renoe fafter bemúthigen - eine Ewigeit,

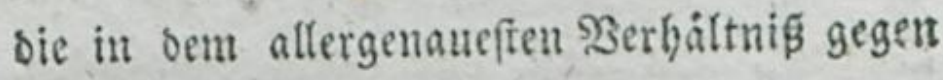
Dab, wab unb auf Eroen begegnet, frefgen wirb.

Der triffende Yresiger fpreçe mit bem Eeibenten bauptfád)(id) aus cinem vierfaçen (Befichtêpunct von ber Llnfterblidfeet. Dars aub follte or zuerfi bie füpe froffinung f́dipfen, bie Dumfeltheit ber $2 B$ ege, welde bie gottliche 2 orfelfung mit ifm gegangen ifĩ, beffer verffelgen zul lernen. Szoffe ges suldig, erwarte ben gewiffen leffrer, ben Ioo, uns bete Bjott an! Şier ift গachts bort Ridit, Gier Stúlfwerf, burt \$ollfom

$$
\text { शु } 4 \text { mess }
$$


menbeit - aे weyten s if bie Erfeşmg Alles bes Hebels, bem unfer irrbirches feben

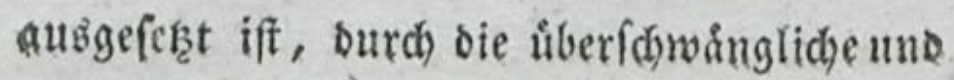

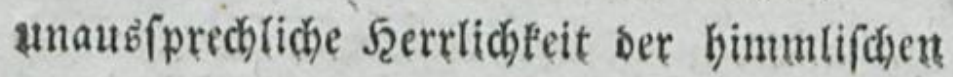
Frenbe! Dein rauker $\mathfrak{W B g}_{\mathrm{B}}$ bat gewis ein Enoe; eb fommt eine 3eit, menn $D u$ in Deinem SaterIande angefommen bift, ba Du aller Sefdywerlidbeiten, unter denent Du feufzeft, hberboben, úber alle Szinders niffe, die Du auf bem $\mathscr{B}$ sege gefunben, bite filer bift. Sn beinem \$aterlande find teine शुúften, Du wirfi nicht mebr foumachten.

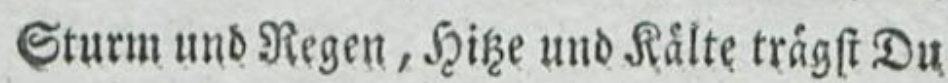
bijer - bort aber feine Stúrme, fein uns genitter mebr. - Dritten s: bie Şbrift serfidsert unb an bielen Srten, baz Rraap. Ser fúrftigen Bjlúffeligteit werbe mit bent bier erbulbeten \&eiben im genauen \$erbåt nif̧ fteken. Die mit Thrámen fäen, were ben mit Freuben ernbten. WSeinent wane

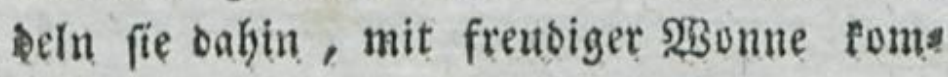
men fie wieder! - Biętenb: Der Zroft ber Emigfeit verfpridbt uns eine fols de Erlofung von ben Hebeln biefer \{ebenz, weldie 
metche mit unferm Dafenn gleidjonuernb if (5ine Errofiung, Die uns allex Jurdt yor fưluftigen Sdbmerzen úberbebt, uno die bellefre

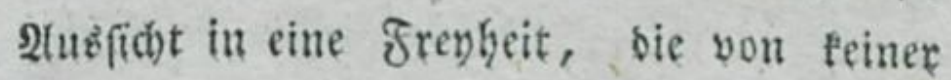
शotb weí, gemólht. Sch faun ben trufts rofent Eltern gewís einen Tag verfpredgen, ba fie alle ibre Sinber wieber befį̧̈е wer= Den, bie fie 2 flle viefleid)t ju frúh, in ibrer unverborbuen Hnifunto vertobren, bie fie auf emig mieber baben. Iक fann bem Sirans ten, ber unter ben hårteften2tngriffen feines Rórpers vielmals ben besten Theil feines \&ebenb burdjeufzen mußs, eine seit ber Ers quifung verfpredgen, wo ihn Gott oon als tem llebel erlofet. Sh. fann bem Teibenden Tugenohaften ein Seben verfprechen, wo bie

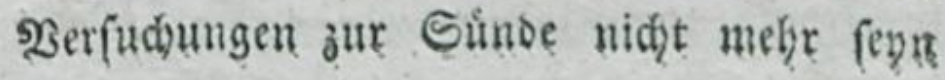
persent.

6.

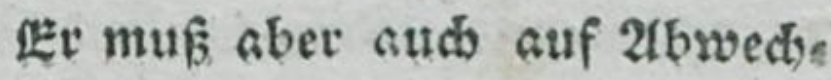
feltung felben. Daher wito er es felye empfeblen, bald surd Sortefen frarter in bie Naterie fohlagenber Stellen guter Sd)rifs

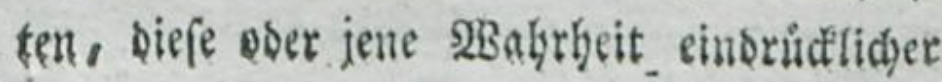

शु 5

แน 


\section{4}

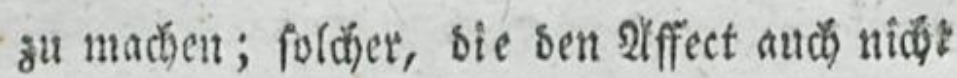
zu farte erregen, Den Siranten zu frarf ers S4hittern, fonoern, wie bie Edyriften eines Toblexs, milo bahin fleepen, fich eins

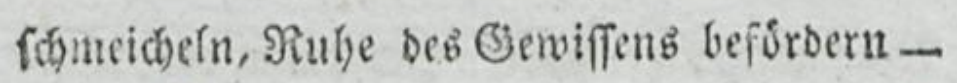
folde, beren es freylidh eine gto (ze Nenge gibt, whiroe ich fir die be feten zu diefem aweet balten. \$3ald burd bs e bet h, biefes frille Erkeben bes

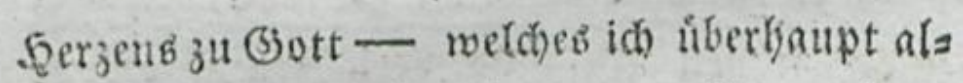
fen feiberiben redt finte anpreifen mirbe, Da man fich faum ein fréftigers 2 erubigungss mittel senem fann - Bald ourd) Ge fang. NBer fenut nidft bie Nacht ber Siefigion, beglcitet von oer gemenhten Nhe fie uno yon des gुfalmb beiligem frluge! Des

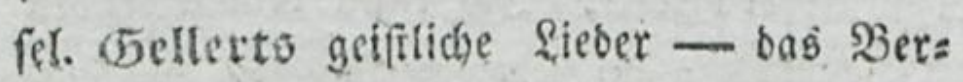
(inifale शeute (siefangbuch. - S. Jout? nal fuir presiger ah. 6. $385-432$. - virill e o moraliche Edildocunger Ih. 3,646:848. Deffetben MTosbeis mi个due driftidte Eittentelyre. Th. 6, 524. forg. 


\section{4. \\ Heber Den Srantenbefud).}

Srante Freunbe in einer guten 2 thofids

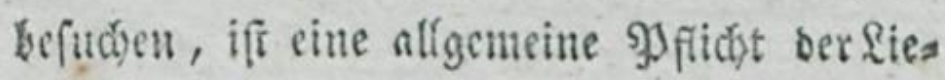

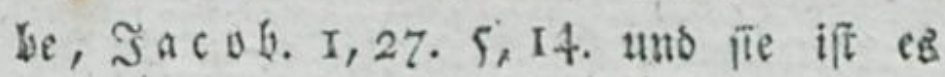
nod) mebr fiur ben \$ुreoiger. So wobr bie

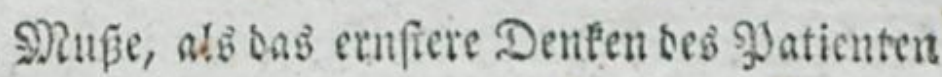
gibt bem જुresiger eine vortheillyafte Gelegens Geit, forglid find fie ifm nud eine Ant ber (sins labing und 2tufforberung, bie 2fufmerffamecit unb. bab $\mathfrak{z}_{2} \mathrm{r}_{3}$ beffertben in biefer guten Rage, mit einer angelegentlicken $\mathfrak{B}$ etraçtung uno

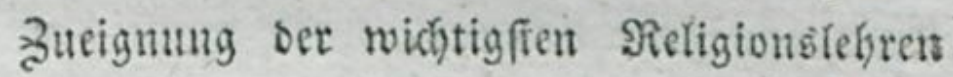
zu befdaftigen. Daher follte ein Reyrer ficty ano feinen ltungang mit Qfinbern fo anges nelym madjen, daf fie von forloft in foldten cinfamen unb fitlent Stumben feinen Befuch erwarteten und verlangten, ober bod) wenigz fens biefen framb aud ungemfen nidge 4ungern fâben. 2foer eben besmegen, damit man nicht tiber ifhn, afs cinen 2logeoronez

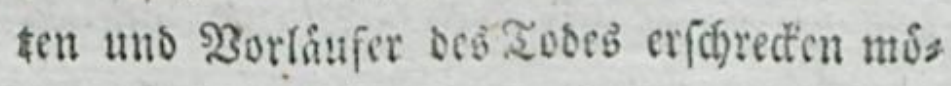
ge, wirt er, fratt bes Ceremoniels eines 2tmts 


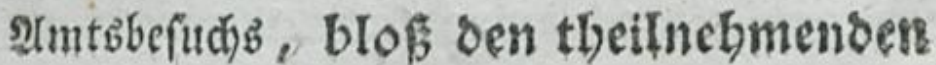
freund madien!

Eine Reget, bie jungen গুyredigern nidbt genug zu empfeblen ift! Daber mus man nicht gleid yom Tobe roben. Er mü die Sselegenteit abwarten, bis er in eill fols (b)es cruftbaftes (sefprách fich cinlaffen faun. Der Sुatient nü erf̃ ein Bntrauen zu iøm befommen. Er mus oafúr forgen, oáz ber Siranfenbefud) nidst nur un(d)dolid), fons bern aud) fo nikglid) unb beilfam, alb nut moglid, feinen Suboirern werde. Daher mus er fie in Sffentlichen Sortrågen uns. Eate, difationen ofterb belebren, woju bergleidgen 20cfuche bienlich find ; aber er mus fie auch vor cinigen gefäbrlicben \$orurtbeilen warnen. Diefe Borurtheile befteben vornebmlid barins nen, baf gar \$iele fich einbilden, WBer nur auf feinem Sranfenbette ben 3urpruch peis neb Seerforgers anodicbtig anbire, fleifig bete, uns bas beilige 2lbendmabl nod eimmal empfange, an deffen Seligfeit fen nidht zal zomeifeln, uen Egriften frebe, ber obne geiflichen 3ufprud 


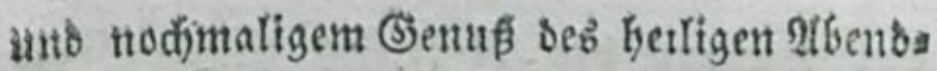

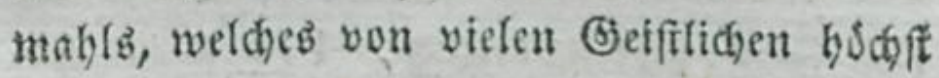
un(d)ifflich uno unrid)tig Der lę̧te Bebrufen nig genannt wiro, verfdiesen. Daber faunt er nidht oft uno nadorufflid) genung oen irs rigen (3)ebanfen rwiberlegen, als ob 5ott in sem lesten 2lugenblict des Lebens mit angfilicher 21 bbitte uno zietse ver. fóbnt werse. Sielmelyr mus er jeigen, onfe ein d)riftides feben bie befite uno eis gentlicbfe Aubereitung zum Tode fen -

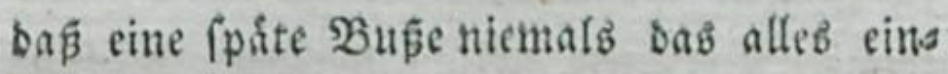
bringe unb erfeţe, wab eine $f r$ úbere ge= nuşt baben wirbe, uno bẩ bie Rranfen febr felten walgre zuverlápige Sennzeichen oet Sefefrung von fid) gábent. - Siebe Tob=

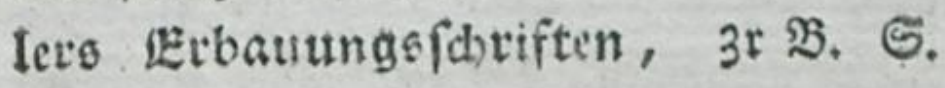
I56. fig. 2 r 5 . S. 225 . fg.

I.

Soll ex fich abet aud wot $\delta a$ p wo er nicht germe gefeben meroen mógte, obne ziuf auforingen?

शur in ben Fallen fann auf eine gute 2(rt ber \$efuch gerwagt werben, wenn

Der 


\section{8}

Der Lebrer sie zistung cincer Gect: fir feir notbwensig, bey dent $23 e$ wustpeyn feiner eignen Gefartatids: Feit aber, Gemütber 3u geroinnen, fir walsercheinlich batt. Sonft breibtz Dabey, Richts mus freyer feyn, alb Reli= gion, uns शidgts Ginbert bie Frudbt einer nod) fo beilfamen Sorffellung fo febr, alb bie शthgeneigtheit gegen sen शiebenben. - 2tud)

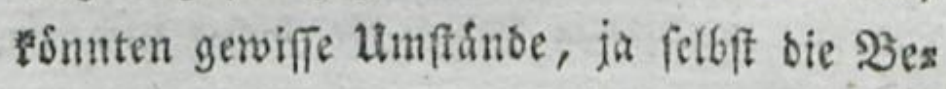
fonaffenbeit der Siranebeit, bie Entfermung frember গु)erfouen nothig macken. SBentigs. frens begefte ein Syresiger ben einer fordert Uinzugangliçpeit feine linterlaffungsfinbe.

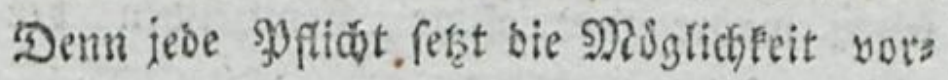
nub. Sn biefem Falle fann fich ber trene Sehrer berubigen, baj ber Sirante Den Ratlf Gottes von feiner Selfigelt oft gentig if:

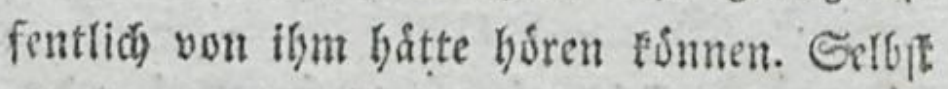
Die Ifpufter haben fid nie 2tnbern wiber if)=

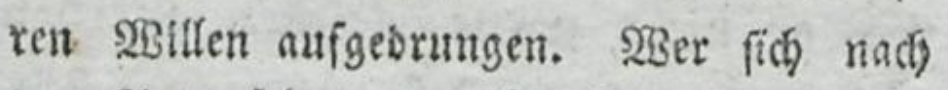
bem $2 r_{3}$ te fehnt, ber lífft ifn rufen. Der Eechtfonaffene Mann sringt fich Zieinems a.uf. 
2.

Wie verboilt fict bev Presiger in ser Zirantenftube?

Srbentlicher solife find firante als Sraurige uno ourch ibre @age uno sngfiticie Erwartungen Bcunrubigte auf Das fanftefte aufzuridten. Daber tritt or als fanfter 9) Renfdenfreuno in sie Stube. ") Diefe 23 cmifung mup barum bie erfe fenn, wit fonfe oas aerfereute (Semuth ben geiffichen

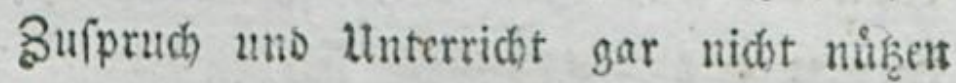
fann; Dann nber aud) Denjenigen Defto lies ber als einen theilncbmenten Jreunb anbšs ren wiro, ocm er feine शiuhe zu veroanfen bat. Uno bis bieber mus er fich vollig nach feinem Syatienten, finer Denfungs, uno Gemutboart, uno nol felbif bisweilen nad) feinem feltfamen (sefdomact, fowobl was Das MRaterielle als Formelle ber criten uhbaltungen betrifft, boquemon, uno foiga

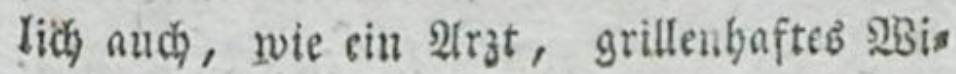

bers

*) Nutr vermeibe or yor allen Dingen alle 2 ffectation uno aud allem Sdein berfelber.

23. 
Deriprectien gedulbig ertragen. Ert wito beth

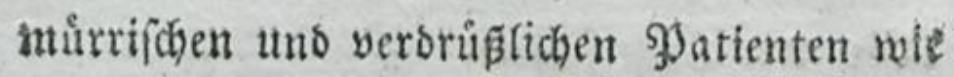
ein verjátelteb Sind bebandeln, oder ifint nachgeben, bamit er felbft balo folge turo gebordye. Dent gltes fommt auf bab gute 3̧utrauten, als auf bie braupt fache ber (Eut, an. Siefle D. Mitlers 2fnleitung. \$.94. S. i72. folg.

$\therefore 3$.

Goll ber Dresiger Diejeniget, weld)e wegen des 2 fusgangs ibver zitants beit gat su ficher fino, ourd eine warnung, oser wol gat trautige 21 s fuindigung oer bedentlitien uns ges fâtuthen Unftande ibrer Litrantbeit geflifferdich tunubig macten?

Illtes wobl erwogen, bin id ber Merys nung nid)t. Denn nid)t zu gevenfen, ons er Daburch bie Zutueigung bes̉ Jatienten, ber Eeçtere aber felber bie, jum ernften Denfent

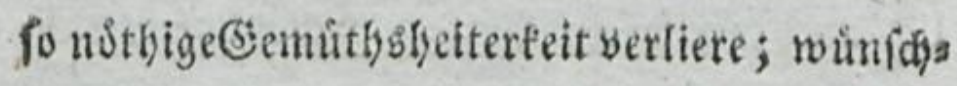

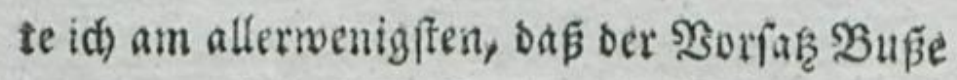
fot thun, eine Woittung der Todesangft feyn mogte! Sif es nidt unenofid beffer, Diefe 


\section{$24 \pi$}

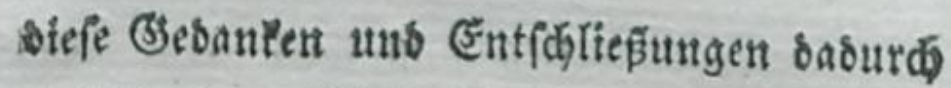
auf bie freymilligfte 2 lrt zu errvecten, bafs man fie überzettge, bie Sambe zu baffen uno bie Tugeno lieb ju gewimnen, uno ber $\mathfrak{B e y s}$ fall unb oie (Snabe Gottes mit eignem $\mathfrak{B}$ es wuft fenn zu befirzen, oab fen ber erfeভdfritt zu

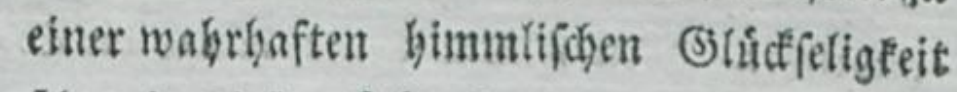
fora in siefem \&eben? -

शach meiner Erfabrung arbeite ida

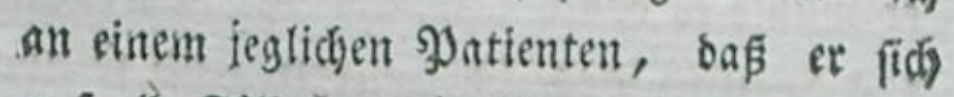
auf alle Falle vernutnftig vorbereiten, unb alfo biefe Atuffordrung Bottes fo weife bes

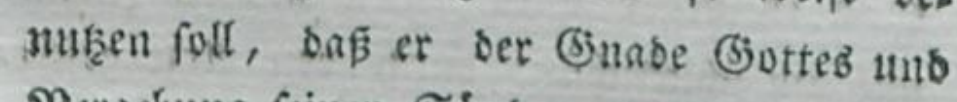
Bergefung feiner Sinden verfichert werbe. Dann follte er ben 2lubgang uno bie 2tba fidgt feiner Siranflyeit bem ङott liberiaffer, ber fie beffer fábe! Sdankte er ihm fein seben,

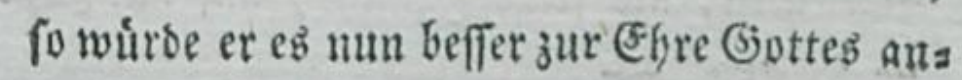

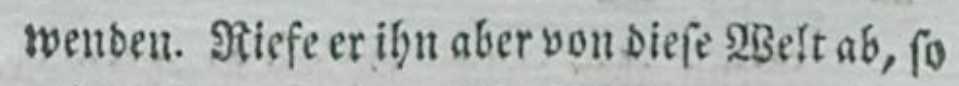
wurbe ihn alsbann biefer Schnitt nie gereuent. Everfdrecle id feinen ourd) bie 2Intúnbigung

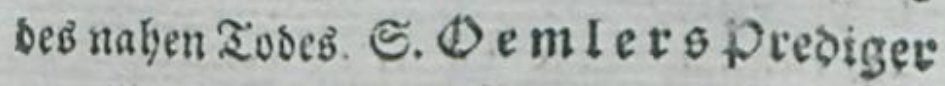
am zitantenbette. Theil I. D. Millers 21nleitung. 9. 64. Seite 173, fg. 政 


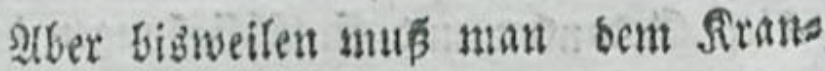
fen fein wafres Enbe antundigen, banit er

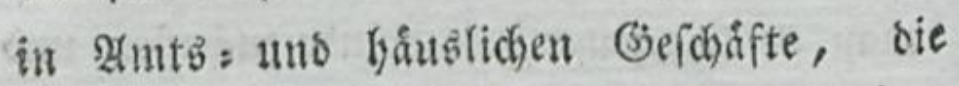
mod) nstrigen 2 erfugungen treffen migeDiefer Grruno beneif't viel, mur nidst, baf ber Iुrebiger ben unangenebmen 2tuftrag

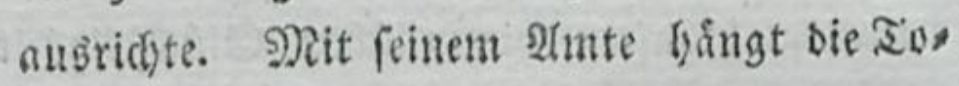
besbotfdaft von feiner Seite zufammen, uns er mípte es baher hidgfents als vectraus tet Sreund des Datienten uno feiner familie thun. 2allein eben besmegen taun es beffer burd einen andern Freunb unb am fobidlidjfen burdh ben 2 razt gefches

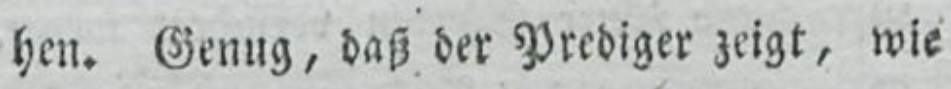
man am ficherfiten in biefer uno jener 23 elt

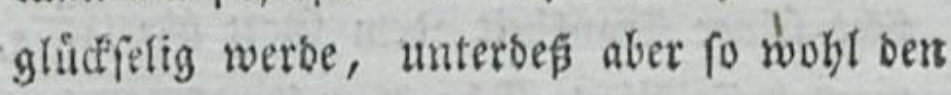
Jatienten, alb feine Jamilie, auf alle Jălle vorbereitet. -

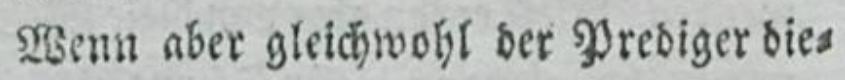

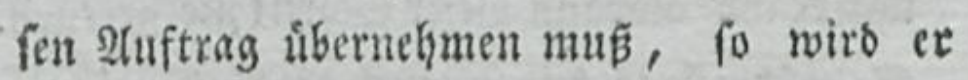

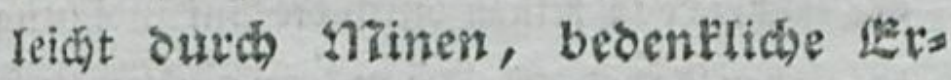

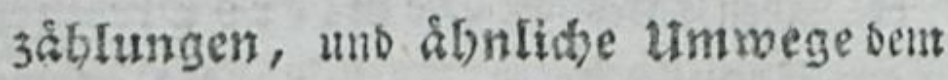
stranten ober feine Battinn fo anfmerpian

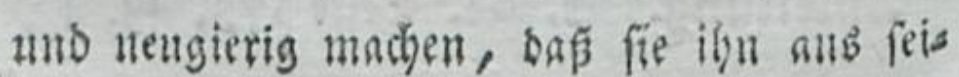
nest 
nest Cebanfen, yon dem ลuftanbe bes गुatien. ten fragen, uno oann fiebt ihm freylica

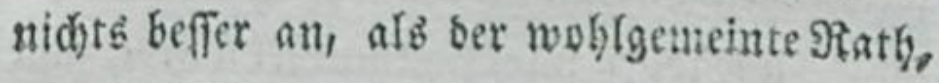
Dab Sidjerfte zu wáblen.

4.

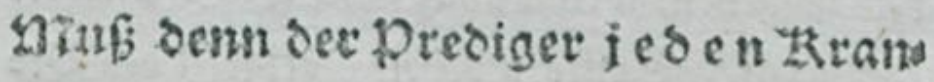

sen, 3 su sem ev getufen wird, befutien?

f̧ier fint wobl einige 2ubnabmen zमs madjen, wenn 3. (. Der Datiente, in einer bigigen Ziranzheit, oen Gebrauch pe: net Einne vertobven bat. Denu bier ift feine (jegentwart offenbar vergeblich, uno er felbit hegiebt fich ofne Noth in (B)efabr. Fur mufi er auf cine fanftmitbige att und mit 2fnfubrung feiner Grinse sen ver: Iangten 23ertch ablethnen, weld ifym aud woht nidht ůbel nefmen wirb; wents

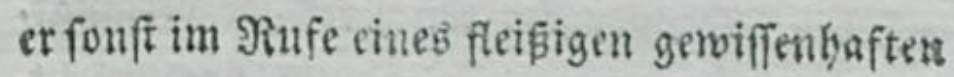
Seelforgers bey Der Eemeinbe fielyt.

Mian verlangt von itm aud of saker oem Gterbenden nod einjegnen foll. Slber bat er feinen şerffano mebr, fo ift es Aberglaube, ben aber ber જुrebiger จurbaus nid) befordern mus.

\& 2

हซ 


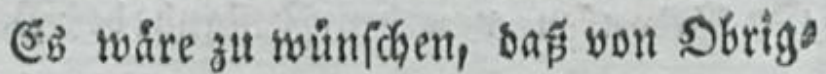

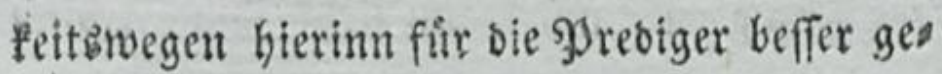
forgt, und ibnen nicht zugemuthet whirbe, zum Unglicte ifrer Familien zu anfectenden Sirans fen jul geben, weldje ganz ohne Shålfe 2 Inberer ba liegen. Sollte nicht die Sbrigfeit bes Dr= teb to viele \&iebe gegen bie গystebiger betveifen, Daß fie bafur forgte, Daß gereiniget, unb bie Stube geraductert wirbe? Ifber leiber! wie unbefummert if man an manden Srten, bes fonoerb in Sthoten, umbie armen গুorediger!

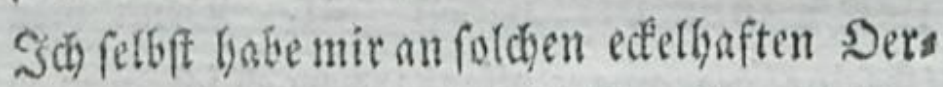
tern etlidyemalgr die gefăbrlidffen Sirantheiten ölgezogen. Nan follte bod) wirflich aufmerf famer anf oie \$ु) rebiger werben! Menfchenlies

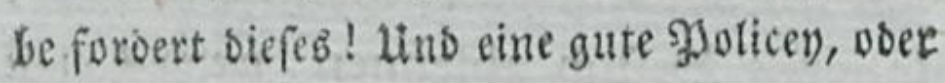
eseroentende Sbrigfeit, wiro biefe झुflid) ems ¥finden. Demn wenn ber פyrediger verlangt, tho nidgt gallz unwiber(pred) fich yorberfiebt,

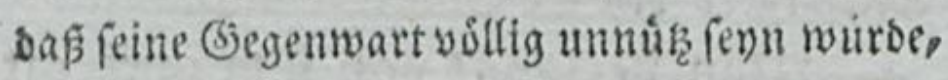
fo if erverbunben zu fommen; felbf zu গुefizeis

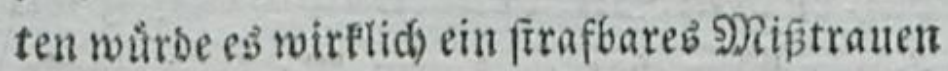
gegen die \$orfelyung fenn, wenn er vielleid)t

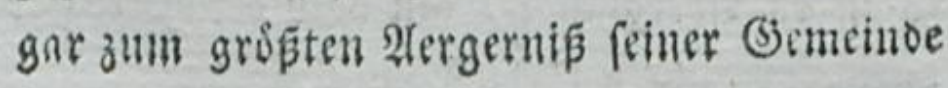
flichs 
flicatig werben molfte; aher es iff nud গুo ficht ber Sbrigteit, für bie Erthaltung Des Rebenb oer פyrebiger zu forgen, zumahl on ibre armen 2Bittmen uno $2 B$ ay fen fo wentge Lnterfinbung und şerforgung fintor. Shl fegne das fons unb biejenigen Stábte, wo Sbrigfeiten bie

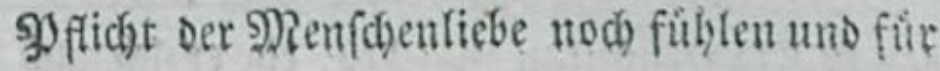

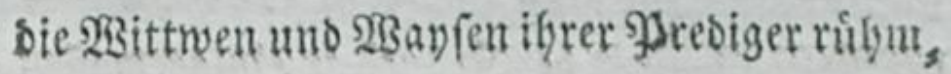
Iid) forgen! - 2tber manther fand uno man=

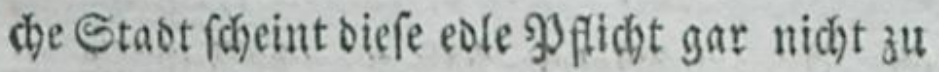

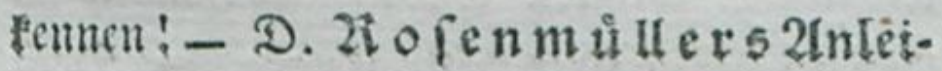
tung fir angebende (5eiftide. $\delta$. IIS.

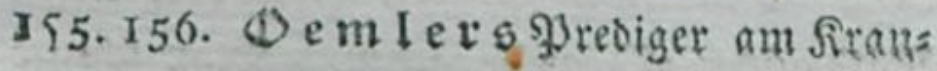
Fenbette, Iter इlycil.

$$
5 .
$$

Denu man fid vor sem thranteatbes (iid) ectelt?

1. 283 er fid bem geiftidgen Stanbe gewiba met bat, mus fich notbwenoig erf vorber gez prifft baben, ob er feinen unberminstid)en Eclel empfinbe, in allerbyand Siranfenftuben ju geben. Er mache daber alb Eandibat \$ro= ben, uno lerne fich in Diefen Studfe fentur.

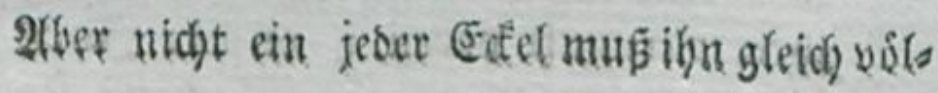

$$
\text { Q. } 3
$$

〔ig 


\section{6}

lig abfareden ; er fann ifn vielleicit nad) uno mach) Durch (jetwobnheit verminoern. Sollte er fich aber, nach allen gemadjten $3 e r f u c h e n$, fdilechteroings zu biefem (sefchåft unvermós gento finton, fo fudbe er lieber ein Sdyulant.

2. 2luch Der Jerzbafte fen nidst fo verwes gen in firanfenfuben zu gehen, ehe er fich nach

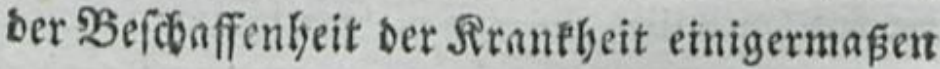
erfundiget hat. Die betrúbten Folgen fols đer Unvorfichtigfeit- Gat manche Familie zu ilyrem grópten शachtbeil erfabren. - Dock fann iø meinen jungen 23 ruoetn biefen

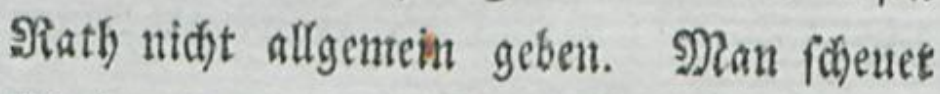
fid) immer vor einer Sirantlyeit mebr alb vor ber anbern. Daber iff ę oftmalb beffer, wents man oie Art ber Siranflyeit niø̄t fo genau weip.

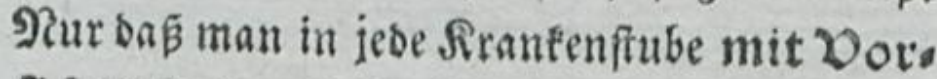
fichtigfeit und 23ebutfamfeit tritt. Bey bişigen fiebern mus ber \$resiger mit bem Satienten nie allein gelaffen werben. *)

3. Ney

\#) Das fidberfte Sennzeidten bey forchen Siranfen, onf́ fie feinen recbten Gebraud ibrer sermunft baben, if biefes: Thee atugen fegenftare vor fid bin; fie

bIeis 


\section{$247:$}

3. Bey gefiffrliden Befudient laffe er worker ráudern, wem! moglich if ; er vers forlucte feinen Speidgel; halte fid hernad) ets wab langer in frewer \&uft auf, uno wedfele

\section{4}

zu

bleiben aud nidht Iange bey einer Gade, fonsern reden gleids etwas anbers; ober fie fitweigen ganzftill, man musibnen die 2fntwort ab. Iocken; fie seigen aud eine gemif fe (5leich)gultigleit gegen ras beil. abenomals and fommen oft aus ifrem 23eidtformular beraus. Solden habe ich bas heil. Hbenomabl nidat gereicht, uno bie Erfabrung bat midh and in meinem $\mathfrak{X}_{3}$ abue beftatiget. Sino foldje syerionen mun wieber gefund mor: Den, fo baben fie es̊ nid, eimmal gemust, Dafi idh fie befud)t bube. Feier febre fid) ber Syrediger an bas bitten ber Ulmftehendent gar nidht, folden Merfonen bas h. 2lbent= mabl ju reiden. Denn fonft befidut er

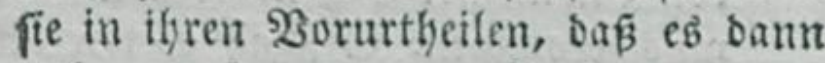
recht gut fey, wenn oer झुatient nur ons beil. 2(benomabl erbalten baitte. Jch babe siefer 2 itten uno Fleben nidbt gead)tet,

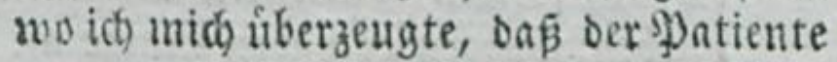
sen recbten freyen (Gebraud) feines $\mathfrak{B e r}_{1}$ ftanbes niät batte. Sie haben es endlid' felbit eingefeben, on B eb umfonft genefen

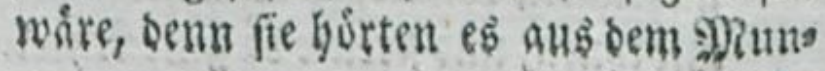

of 


\section{8}

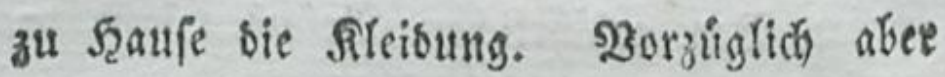

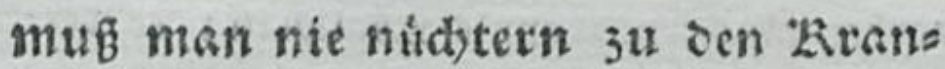
Ien geben; ben Shutb vorker mit Wseinef. fig ausfpulten uno bann uns wam cin Glas Ibiat,

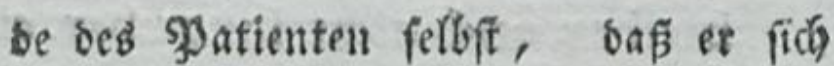
nidbt erinnern fonte, baf idb thu bes fubst batte.

פBey foldsen झुatienten mus aber der S3rebiger oie grofiste $20 r$ ficht und 2 ehut= famfeit anwenben, benn er ift bey Soldgen in groper (5efabr. Sir fino Dergleichen Bevfpiele befannt, wo biefer mo jener Drediger burch 2llteration und Erfáred"s nif fich feinen నos zugezogen bat. $233 \mathrm{ent}$ Derগु) oftploficlid) undumernuthet, f fabrenauf,

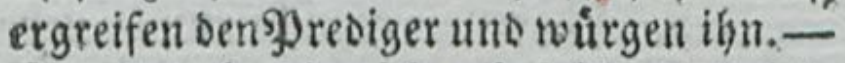
3uerft babe id mich auf folche Iluftritte

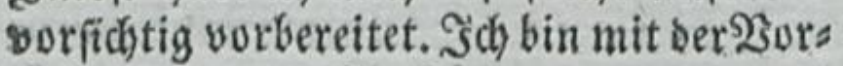
ftellung zu sem \$ुatienten gegangen, ex Fónte in meiner Ssegenwart ben Waroxis:

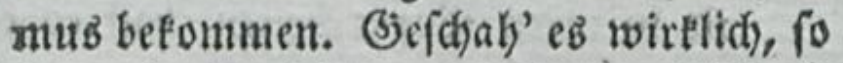
tam es mir nid)t unerwartet, unb ich als terirte mid) nicht bariber. Seernach bin idi) bey folchen Watienten nie allein ges blieben, fonoern es haben sinige an bem Sette bes. Sranten ftehen miffen, bie auf

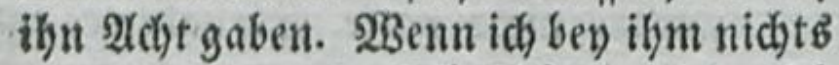
ausirid)ten fonnte, fo befd)afrigte id) mich mit ignen, und ftreute ba gute \&ebren aub.

รัต 
Rlyeinwein mit 20 నropfen હitronenfaft 孔

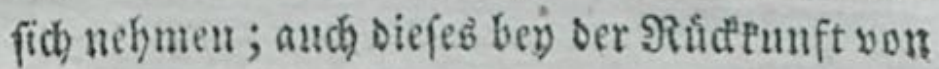
einem foldsen Siranten tbun. Siefes Práfervas tiv hat mir ein gefdifter $2 r_{3}$ t empfoblen, uns

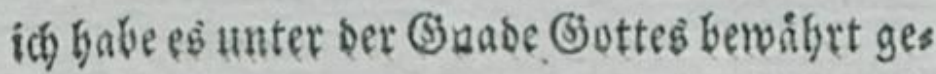

funs

Sin orefyete aber atch sen Stuht, ben fie mir gabell, um miđí) oarauf zu ferzen, mit

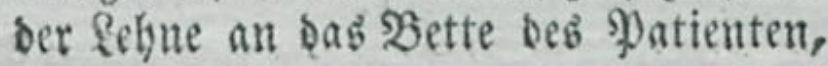
ferte mids nie nieber, (onbern id flanto vor sem Stuble und legte meine $\mathfrak{r}_{2}$ and auf bie \&ebue, gleidfom als ferzte id) mich

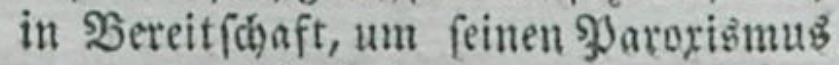
yon mir abzubatten. - - Was madten wir aber bey foidben $D G$ tienten? Ës iftwahr, meine $\mathfrak{B}$ ritser, wir fónnen zwar oft ba gar nichts auşs ricten, und boc miffen wir fommen, went wir gerufen werben. SBir muifien bier ber Sdemadbeit ber Menfien nad)s geben. 280 ollen wir nid)t fommen, fo vers fielen wir in verfdyiedene ung nadbtheilis gellrtheile, bie unfrer2tmteffúbrung in ber Sufunft fódblid) werben f'sunten. Sơns nen wir uns zlwat oft mit bem Yatienten. nicht befdaftigen, fo find Doch andere Giez funde ba, mut benen wir ein23 ort zu feinec Beit reben fósnuen ; fo ift bod) urferbe fuch nid) umfonft. Mit Einem SBorte! 28 ic ergreifen jebe Bielegenbeit mit \$ergnuia gen, und frreuen guten Saamen auf 520 ffa

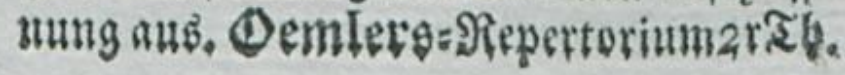


250

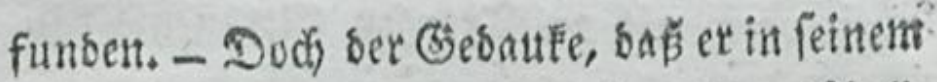

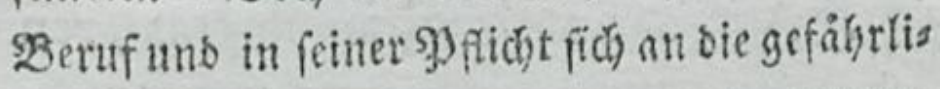
chenDrte begâbe und 3 ertrauten auf oie gittliche Borfoctung, wiro fare genug fern, ifyn vor angfridjen Beforgniffen zu beroblen.

I. Oie s ll gemeinenziegeln bey Sem zirankenbefutbe find folgende.

\section{I.}

Der presiger jieke vor allen Dint gen oie zeforffenbeit oer leiblidjen Zirantheit in Fluge Rerwagung. Siegt ler Matient im Gikgigen Sicber, ober in grofien Echmerzen, fo fann unmoglich auf benBortrag

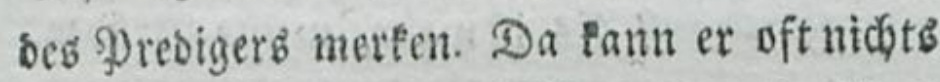
mehr thum, alb daß̧ er in Gegenwart ber $14 m=$ fichenben mit iflu betet. - Nande find jwar

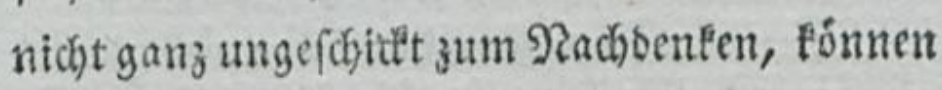

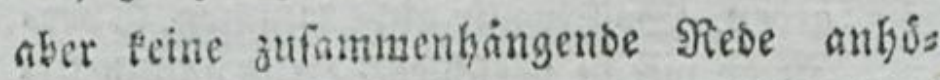
ren, am allerwenigfen, wemn ber \$rebiger ft arf rebet, welciges obnebin in LAran, fenftuben nidjt gefweben follte. - Da (iic) zufanmenbaungenbe Seben gar nidyt ans Siranfenbete fífiden, fo mus oer \$yretiger zatu'ze Detfe uns Sptudide anbringen, 4t16 
ano fich oariber mit bem STranfen, nts Freuns, fatadjetifd befpredjen. - IEáre ber Siranfe bereitb sem Iode febr nabe, fo fann er unt furs ze গুerfe und Sprád)e mit miglidfter Ipplicas

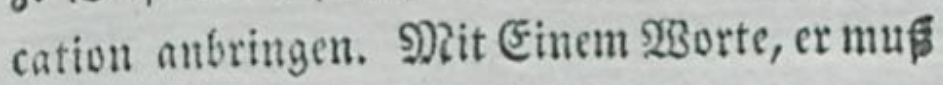
(id), fo viel ilym nur immer mogltad) ift, nads bem Yatienten ridgten, uno bie Uuftånde ber Sirantheit beobaditer.

\section{2.}

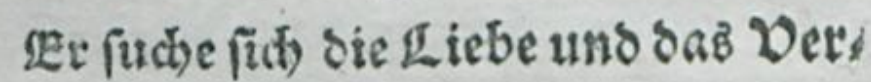
rtauen des patienten 3 erwerben. Demn fona if fein 2efud) fruchtlos uno verges

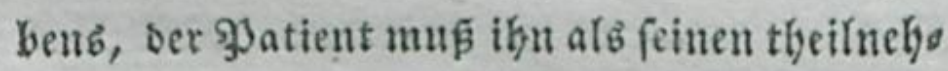
menoen freumb achten, nid)t aber als ben $B_{0}$

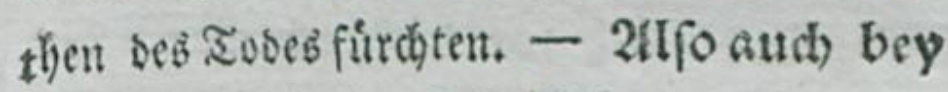
Eolden, die in beimliden, oser gat offenbabeen Laftern bisber gelebt bas ben? Allerbings ! ১enn die unvorfid rigenpoltererundertwafpresigerfdyasen sem patienten an Leib uno EecIe. -

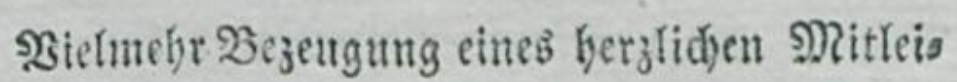

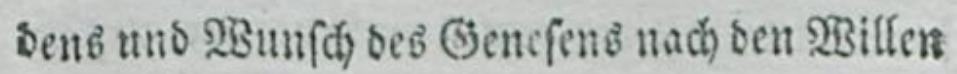
Giottez, wirb ber Snlyalt ber crften Unterrebuns gen feyn miffen, nit was furłperfunen wan aud 


\section{2}

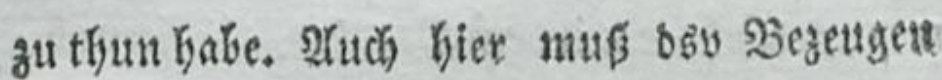

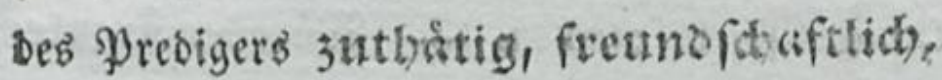
theilnebmens feyn. So fieft und hist matt ihn úberall gern.

\section{3. ,}

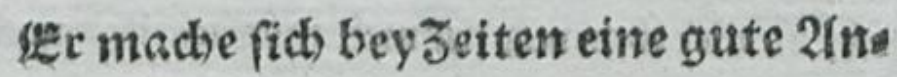
$3 a b l$ von wict)tigen Epruichen und Lies dern bekannt, oie fidy auf allethante

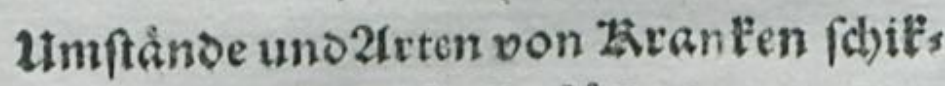
ken, oamit er auf alle Sallle beteit pey, uno nux nicht in bie frothwendigfeit fich) verferst febe, entweder ein Gebet? buch zur band 3 nebmen, oder fich

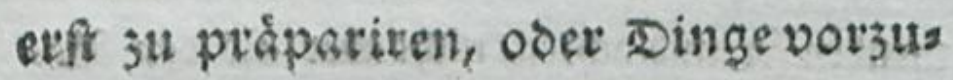
bringen, sie fids gar ntht fibicten. Ein gुrebiger, ber Gier Hnwiffen beit verråth, futbitet

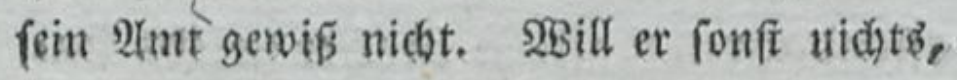
als ignr \&ieber sorlefen, und gebrutte Biębete, fo braudjt ber \$atient ihn gar nidjt! Dent Diefes fơnnen beร গুatienten Freunoe thut. \$on folden unerfabrnen şrebigern fommt

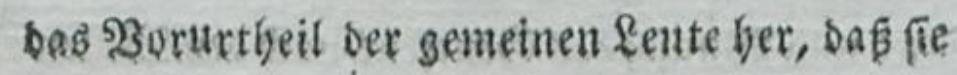
zu bem \$rebiger fagen! ter foll 3 ul oem siranten fommen um ein bişchen mit ibm 3 beten.

4. 


\section{3}

4.

Lange 3ufammentiangense 2iesers muf matr nie 3unåa) für oen Kiran= fen balten, es fey dann, oaf man es 3 th Lerbautung uno Rithrung oer $2 \mathrm{f} n$ wes fenoen fưr nottrig balte. *) Sit ber

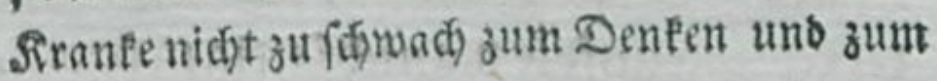
Sieben nid)t unaufgelegt, fo bilt man eine 2 (rt Der Lnterrebumg mit ifm, mit untermifden Sprídyen und 23 serfen ats erbauliçen \&icbern,

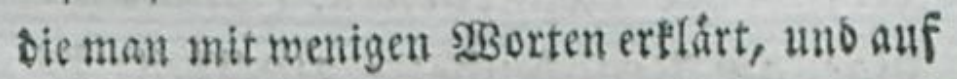
feinen Suftant anwenbet. S. D emlers Dreoiger am ziranfenbette, 2 ter bis $5 \mathrm{r}$. Theil. Der Befdinf bey einem jeben Befudje

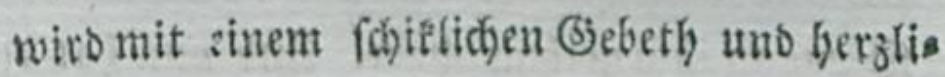
Gen 2 sumfde des Seelforgers gemadt.

4.

*) ItGer nuch für Diefe, dínft midh, fino lange juf folde en Srt faft immer úbel angebracht

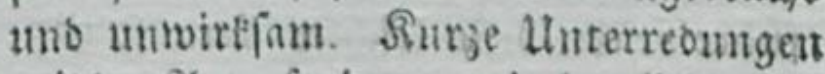
mit ben2tnmejenden - ein herjlides (Ers mahnumgs = Ermunterungs $=$ ober Trofts wort, bem ein Epruch ber sibel Siraft,

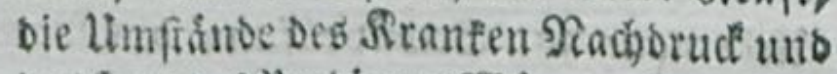
Dณธ่รุer gemes immer frudibarer, als bie besten zufammenbángenden Pieden.

23. 
5.

Damit ev Sen tzuanen 3 um 2 tescn buinge, uno feinen moraliften $\mathbb{C b a r a}$ : ter fennen lerne, weldhes 3 u einer beils

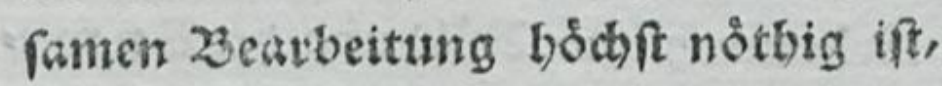
fo fübre er ibn gleich bey der erftenlins tertesung anfoie manich faltigen Wob is thaten, die ibm Gott von feines tainds beit an etroiefen, uno aufoie beilfame 2ubfid)t ser zitrentheiten. (5. Ser pres siger am Kirantenbette 2 ter Theil) Dies

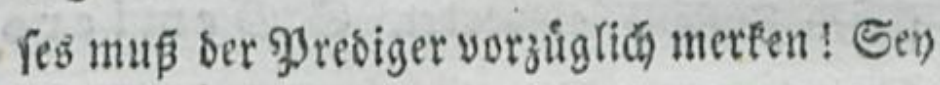
nun ber Patiente, wer er nur immer fer), fo

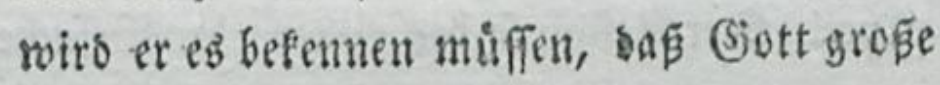
Dinge an ifm gethan habe. Hno man hat er Daterie gemug, forvohl zur Unterrebung, als zur Selbftpriffung.

(jeferet, er glaubte, oaf fein \&eben ein felyr muibfeliges und elendes gewefen fen, fo fúgre er ibn defio leichter barauf, baj biefę

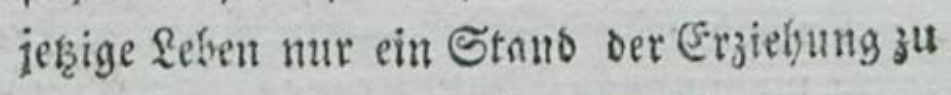
einem beffern alffande fey. - Eo fann ber Ulebergang z!!r Selbfiprififnng beb \$atientent auf eine leid)te und ungezloungene 2lat gemacht werden.

Die 


\section{5}

\section{Die Ectbftprifung muF bem}

Zutanfen auf alle nur moglibe 2trt ers Ieichtert recren. Es iff ein groser Fefler, wenn mandbe \$rediger jeben \$atientent auf eis nerley 2 trt bearbeiten. Denn wie verfohiebenints

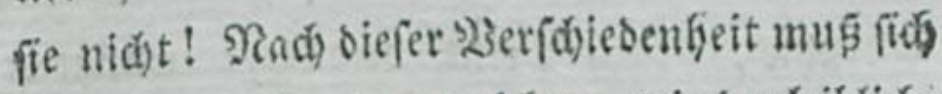
DerDrebiger ebenfo gut ridten, wie ber leiblidge

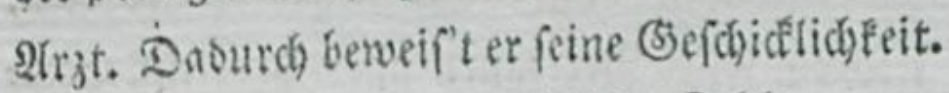

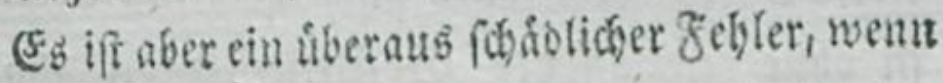
mancheyrebiger alle ifre Patienten ohnelluters f(d) aufoas Deroienftebrifti bimveifen. Dief find, wie beit Eobler fagt, sie rifjets beraufitenoen 21rzeneyen der Eeete. Diefe $2 \mathfrak{A r z}_{3}$ eneyen finb jiemlich angeneffm uns Inbern bie Sd)merzen eine Betitang; ber \$as lient bleibt aber im Grunbe fo frante alb vors Her. - Die befre Arzenen ift, die einen wabren bấ der Sunde und eine 2tn. nebmung des ganzen fEvongeliums, mit einem fid felbft ridtig Eennenoen berzen, erbeifiot. Die $\mathfrak{2}(r 3 e n e y)$ if fur bie Meifen wibrig uno mad) vieffeidt anfangs sie Schmerzen nod) beftiger; fie heitt aus bem Brunde. Durch bicfe Selbifprufung folf ber

Sirama 


\section{6}

Sranle verwabrt werben, baß er fich nidjt yot falfoben Brifungen taufchen laffe. (E) muв ifgm baber gefagt werben, Daf meber áuffere

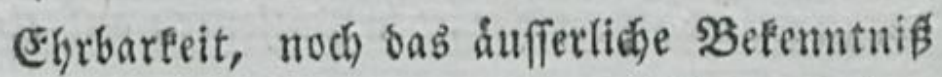
zur wabren Sieligion, nods geviffe angenebme Befible, nodi cine befondere emfigetet Seobachtung áfferticher SInbad)tb̊tbungen,

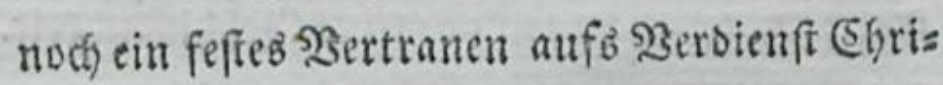
fit ohne gebefferte Gefinnnumgen ifh grundtid berubigen burfen. - Einjeber rechts

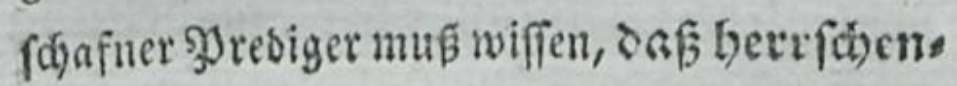
seliebe 3 (5)t und gendbafter beftandiger Steif sas $23 \delta$ fe 3umeioen und ourbgangig rebt 3 u. thun oas einige 3uverlifbige tiennzeis d)en cines wabren Elaubens uno folg: Iid) auch bes Gnabenftandes if:. -

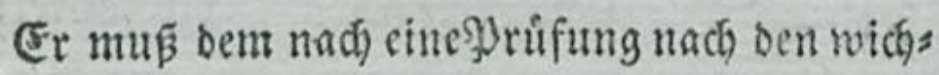
tigften $\gg$ flichten auffellen, wobey er fich bauph

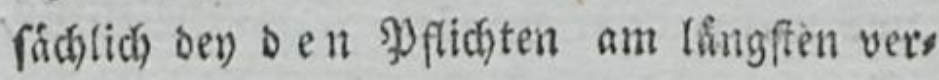
weilen mus, bie ber Syatient entweder am meis ften zu libertreten Belegenbeit gehabt, oder

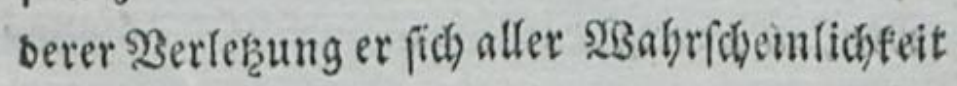
nad) wirelidy fobuloig gemadyt bat. S. Pves oiget 


\section{7}

Siget am Lrantenbette. Theil $2=5$. D. Zo fenm th llers 2Inleitung S. II9.

7.

Da bem

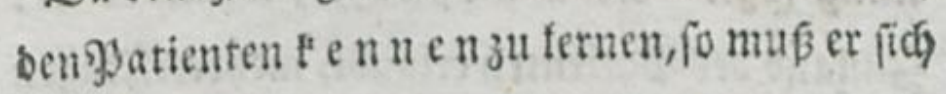
bemútyen, ifm ein freywilligeb $\mathfrak{B}$ efentnif abs zutocten, ob er fidi getraue felig zu werbent ober nicht? *) worauf fich feine be

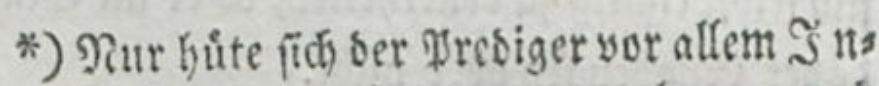
quifitorwefen; er verlange uno bringe nie auf ein fpecificirtes Eunbens betenntnis; felbfi bann for bere er bab namentlicbe Sinbenbelenntnis nicht,

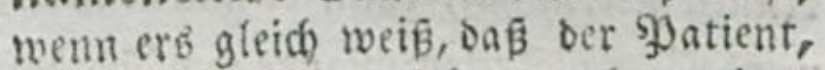
burfil diefe ober jewe Sunbe, fich verging.

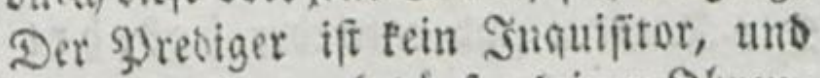
wir \$ु roteftanten bebirfen teiner Shrens

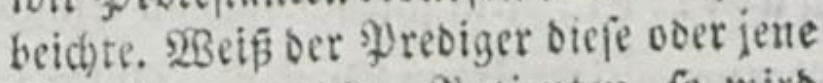
Bergefyng von Dem Yatienten, fo wiro

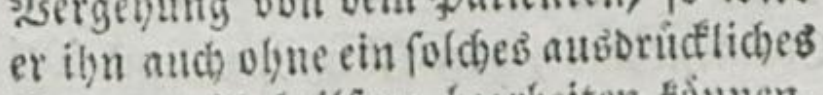
Defenntnif́ beilfau bearbeiten fơmen, uno - vermutbet er fie mur, fo rid)= te er mit 2 SBeişbeit uno liebe feine $\mathfrak{B}_{\mathrm{B}}$ bandung barnacb ein, liberlaffe aber íbs rigen sıllles Dem, ber ba red)t ridetet.

23.

\section{$\Re$}


be? oder woran er zweifele? Jreylith erfiffrt man ba nid)t allemal ben $\sqrt{2}$ erzenछzuftand bes Sुatienten, wenn er gleich feine Soezzensmeys sung fagt. NRan muв daber, wenn eв ber

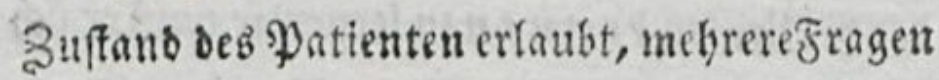
an ify ergehen laffen, fo bas cine Art ber Sate= difation entfelyt, wobey ber Drediger feinen Siranfen immer in fein Şerz fübrt, uno св ifgm fo leid)t mad)t, alb es immer molich ift, iīb fetbit rid)tig zu beurtbeilen, ob er im Stan= be ber Ginaden fey oder nicht.

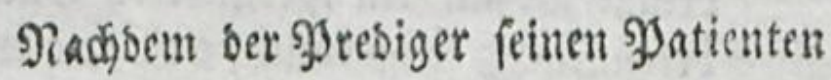
zennen gelernt bat, fann er mun benfelben fei= nembefundern Scelenzแftande gemåв behandeln. Die Siraneen find entmeder befebrt ober ut nbefebrt; jene find (chwach an (Finficten,

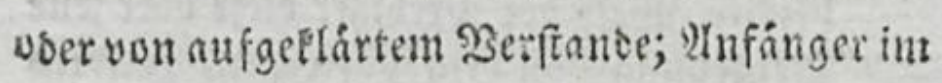
Ebriffentbume, ober Singlinge uno Mánuer in (5brifto. Unbefebrte aber baben fid bisher entweber offenbaren \&aftern ergeben, who fint entweber ficher, ober erwectet, ober fie waren Sgenchler, Eine icoeङsattung biefer\&ente forbert

eine 


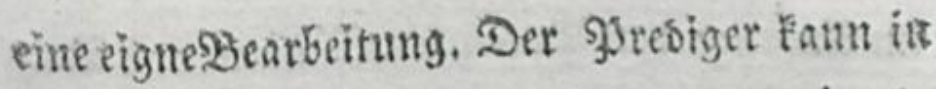
feinen(sewiffen rubigfenn, wenn er nur mit rebs

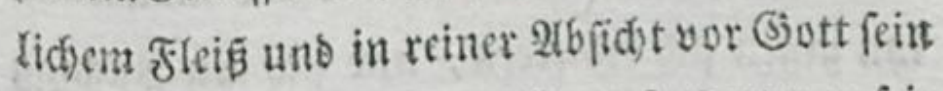
S) Xoglidfres thut. Dem @ott forbert yon feis nenDienern bie đreue S. Prediger am Zirantenbette. Daher fino bem গुresiger, II. Solgenoefsecie lle Ziegeln notbig.

I.

Unbetebrte Erinoer, von roeldee 2irt uno 3 efichafenbeit fie aud immet reyn mo̊gen, muffen vor allen Dinger

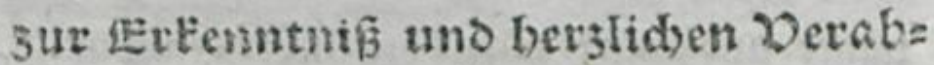
fiscuung ibres bisberigen Lebeno uns ibrer berefósenden bo fen Gefinnungen gebrobt mersen. Diefes ift inft fur bett Brebiger bie fd werfe Ecene. Demn ber Borz nelgme furwobl; als ber Taglofner, ber STBeife fo" wohl, alb der Einfâttige, weís taufenb Shus fuichte uno Entfoutbigungen, bie er mefren theifb fur fid bebalt unb wooură er alle gute 2tbfic)ten feineb Eeelforgerb vereitelt. (5: 3

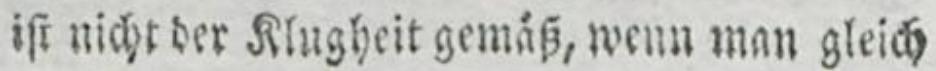
श 2 ints 


\section{0}

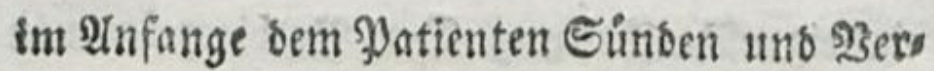
gefhungen yorbalten will. Demn man fann ifin gegen fidc erbittern, uno fich oen zutgang

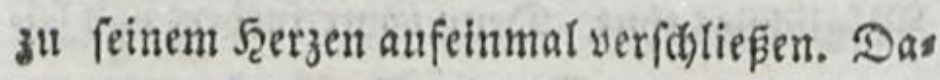
Ger muß man im2tnfange nur bey dem 2fllges meinen frehen bleiben, uno mit webmuthävols Ten Empfinbungen bie grofe (jefabre einer bers fohenten $\mathfrak{a}$ afferbaftigfeit nach bem \&eben faits oern. Nan ubergibt ibm bie gemachten 30 rs frellungen unb Serwiffentrigen jut ungehett. deften Ucberlegung vor Gott; tritt feinem Şerjen bey einem fernern $\mathfrak{S}_{\text {efuch }}$ noch etwas náber uno áuffert etwas deutlid)er ben2B แnifh, zu wiffen, wie fein Serz befduffen fey. - $\mathbb{Z B B}_{3}$ re audi) biefer frudt)tlos, fo ift bann fein anderer Rath, alb bấ man eine oder die ansere Guinse, die offenbar bis liteler bey ifm bertfach gewefen ift, bey ibren redten rabmen nemmen uno it) oann mit webmithiger (Empfinsung uno liebrcis d)er Ganftmutl) die vaterlidie Z3efor, gung um fein eroiges wobl 3u ertan

men 


\section{$26 \mathrm{r}$}

nen gebe, wofern er nicht in fid) getgen wiribe.

)ir iff es ba felgr oft geglictt, wenn ids theils oaso menfdidide berz, in feis nen vergelungen gefdilbert; sie mandberley Gefabren sex Deriundo gungen reutlith vorgeftellt, uno im plutali mit ifsm gereot babe! etwa auf folgende 2 (rt : $2(d)$ ! wir arme श) Renid)en! rie oft thun wir nid)t red)t: (Sjott wem bis

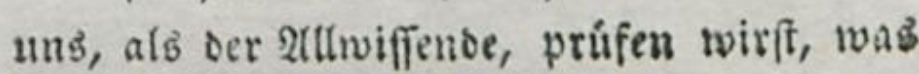
wirft ou aแ ung zu tabeln finben! u. โ. w. Bald werben wir ju biejer, bals zu jener Simbe verfuifrt! -- Daburd habe id ben

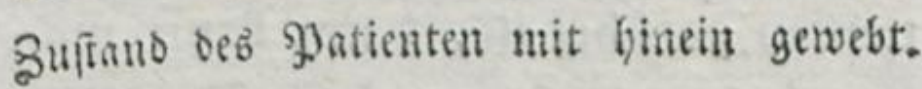
इ⿻一, habe ibn getroffen, uno er fonnte nidit bife werben!

Berlangen, Daß̋ er getwiffe einjelne Sunben auf bem Tobbette befennen foll, wirbe in ben mebrften fallen eine fegr wia

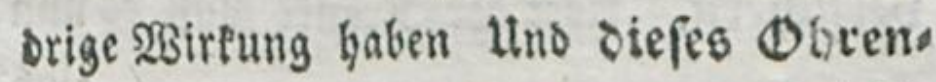
भ 3 beidsts 


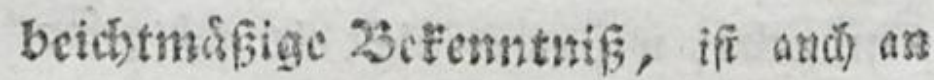
fid) nidgt nitbig zur Beremung oer Sinbe. Ев if genug, wewn ber Sirnte nur hibers

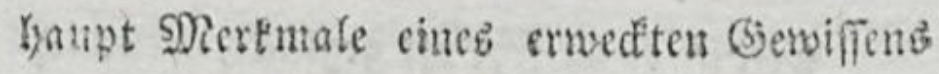

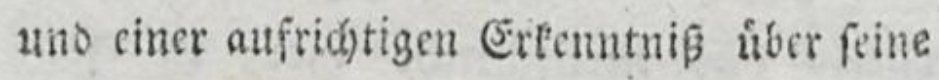
Einben an ben Iag legt. Man muß aud uicht yon bem Patienten zu viel forbern! (5)mug! menn ifm der Jreoiger зum auf= ridftigen Befenntuis feiner Sunben vor (5) o t ermuntert uno ifjn vor aller Feudjes (ey) macboruditich warnt.

2.

If man aus 3uverlafigen ntert, malen gewiß̄, saf das bevz oes Das tienten gevibet iff, Dosn foun mon ifn Gud getroft, auf sie 3 armbers 3 igfect (5ottes uns sas Dcroienfo Cheis fit binypeifen, uns ilsn, im fall einer ber3lichen ungebendselten zietu uิber fein bisheriges Leben, dis Gnade Gottes uns vergebung ocr Eunden

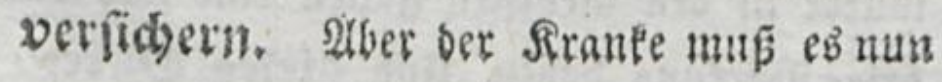

aucic 
aud in wirffichen Proben, fo weit cs mur fein gegentrártiger Zufrand erlanbt, beweis feil, bas eine wabre 2lenbrung feiner (s)efits= nungen yorgegangen (en, unb nod vorgehe. Dafjer mus er alles bas wieber erfeşen unb gut madjen, was fich nur immes gut mas

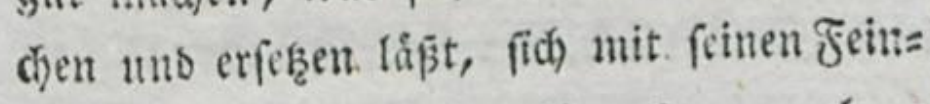
oc aแรึง uns bigher befalteme but refituiren fo weit eb in feinem \$sermogen fteht, bie von ifm

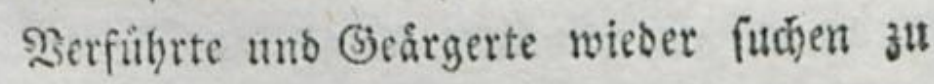
erbaten, uno fo weiter; bod) mus man stlles bem eigenen Gerwiffen bes Errvectten

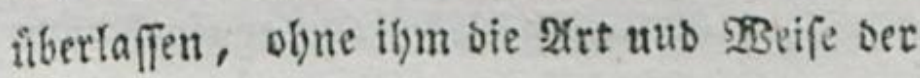
Sieftitutionspflicht zu beftimmen, unb vorju= forreiben. S. Drodiger am Zirantens bette. - Sisie felten aber bie $\mathfrak{B}$ upe auf Dem Iosbette aufriditig fey, ift gants uns Yeugbar. Daber mun fie ber atreoiger be= fonbers sur 2tufrictiglett ermaknen, uns yor Szendetey marnet. S. D. Ro fens

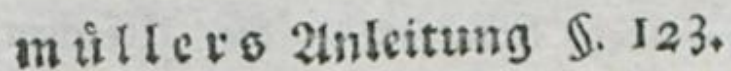

$$
\Re 4 \div \text {. }
$$


264

3.

(1) gleid aud wabte ธIaubige auf die feblex uno Gdwrobbetten ib reo Lebens aufmertiom múfien ge= madbt wersen, fo Kann riefes sod mit weniger limfindnolid)teit gefdues ben, fonderlid, os fie felof ibre mian= gel fublen. Man erfulle tubrigens ibr Serlangen; veriage ifuen Sufprud) uno Şorbeten nidt, wenn fie ç begebren; fileis

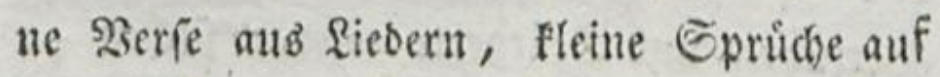
ifren Buftans applicirt, abgebrodbue (s)ebete uno Seufzer, fdicfen fich am beften, uno werben auch bem Rranten am angenelymfent fenn! Uebrigenz laffe man Die, oie ridbtig vor fid) gewandelt baben, obne (bernufd in

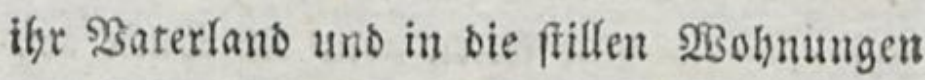
des Friebens von binnen geben!

Sey ber Beurtheiúung ber vielen $\mathfrak{B e c}$ angftigungen, bie man oft erbliffet, fey ber Şrebiger immer befutfam! bie Urfachen liegen meiffentbeils alle in ber Sirautgeit, poer 
ober in bem Rórper. Sie bem fatanifochen Berfucfungen Jufdseiben wollen, if ládgers Iid) und verrath Unverfano und Huwiffen Keit in Seetenerfahrungen. S. D. Mil Ierg 2inleitung \$. 96. 177. 1so. D. Zio fenm it llerg 2 fnlcitung $\$ .125$. Der preoiger am tirantenbette. Th. 2. \$erurfact) aber vielfeidt bie Erimerung einer Sugendfünoe bie Beångftigung, fo bitte ifu ber \$ुrebiger, ifgu bie alweifel feines (S)nabenfanbes aufrid̨tiz zu entbefen. Der

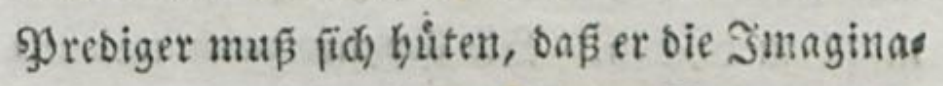

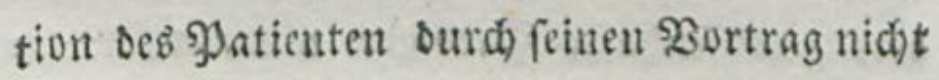

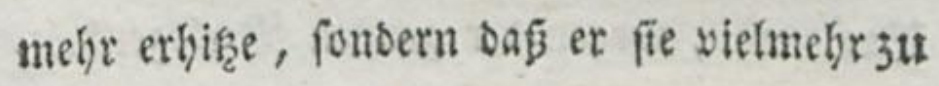
befinftigen fuche.

Dev 3uftrampf ift leere Cbimåre, bern bie 2 bibet weís nichtz bavon. . Er if Narter ber Giewiffen - Edjefe Tiidtung Des Ehriffenţumb - Nange! ber Exfeunto niß̧ in Seclenerfahrungen!

$$
4 .
$$

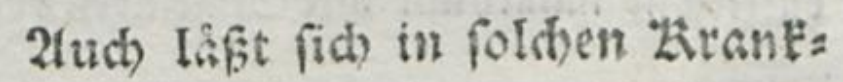
Geiten, oie fïh) plóslid) cutjuinden, ^5 แกเ 
und it einen friben Eos cufo Iofen, oser auch bey febr fomers bafs ten uno สuffert befकroerliden כufât= Ian nidbt oronunge, uno pIanmábig vevfabren. 2Bab faun ba ber gुrediger mebr thun, als furz uno fräftig liber ben গु) ten unb feine freunde anweifu, was fie ibm, wenu er rubigere 2tugenblife bat, vorfagen, ooer vorlefen follen. (Ẻ if betribt, daß́ ba ber झुrediger nufgeforbert wiro, fuldyen গुattenten fogleid) oas abenbs mabl zu reidien. Sob bedaute mich uno an= Dere gerviffentafte \$ुosiger unter meinen Zutoewn, wegen ber gropen నerfegenbeit, in meldbe wir unter manḑenllmfianden beşfarร fommen máffen. Sey Gilabigen uno bey Denen, Die richtig vor fich gewandelt haben, faum man ify $23 e r l a n g e n$ mit sergningen erfiflen. 2rber was follen wir bey Denen thun, bie offenbar gottlos gelelst haben? Itm fich nuffer Serantwortung zu Fegen, Durften fie immer nur igre Giemeinoen von ber eis 
eigentlichen SBefodjaffenbeit uns sem wabren

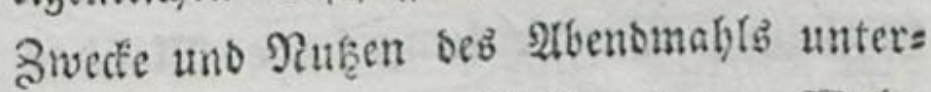
richten, uno ignen liberbaupt ben $\mathbb{R B a b n}$ von oer firaft einer menfolichen 2lbjofution binlanglich benelynen, ifnen aber iberbaupt die fpate uno fdunelle $\mathfrak{B u f e}$ yerdád)tig maa chen. Sabuen fie bab oft unb grumblids ge= mug gethan, umb ber Siranfe uno Die Treuns be beffelben befteben doch nuf bie Eommunion; fo Eann er es, went ser patient nod) Derfano bat, nid)t verfagen, meil ex nut als Werfieug und Dies net ber Firblichen Societit banden, und (id) mur nod feinet Inftenction in ber thirobenordnung rid)ten muf. ङ与 wåre aber langfi notbig gervefen, bafi bie Sonfiftorien uber biefen bebenflicben

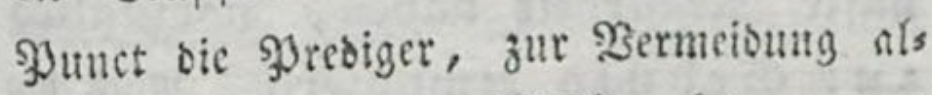

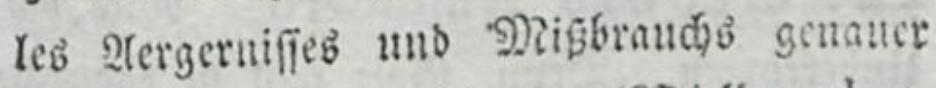
informirt bâtten, ธ. D. ni illetlc. ธ. I79 $=18 \mathrm{I}$. Ueberbanpt iff ben foldjen Rranten, bie in ifrem Reben bie Retigion nidjt getiebt baben, Unterriøt, \$Eetérung แแก 


\section{8}

unb 2 effrafung zu fpat, nuv nicts eine mitlei ige dempfe lung feines felendes an (5ott, surth eine Fuftige fubbitte, und fann man fie gleich uid)t beffern, fo muf ood) oer prebiger biefe Belegentyeit bas z̆ benuzen, baß̧ er yor ben IInmefenden die Ebre ber Religion rette, uno fie baburd) ers

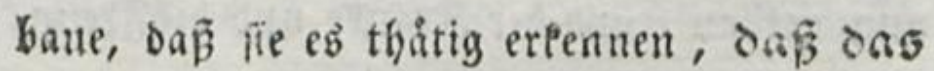
ganze Leben cine would ge Worberes, tung auf jenes erbabenete feyn milfie. 2 Petr. 3.13. S. Dresiger am Latan= fenbette. Theil 1. D. 2iofenm นํ. Ier 1. c. S. I26.

5.

Let muf alle Die, sie eine folmerz: bafte Erantheit baben, vor3iglid 3 เ 5 e o 10 ermuntern. 2(ud) de Unbefebrten. Denn (Sontt will fie oaburds erweden uno aufmerffam machen! Daber gebe $e^{\circ}$ nie mit ilynen jum Sserberten, fons bern zu ifrem Blicfe. Sie follen Gjott nut nidjt auștweidgen, fonbern feine våterliçen 
Ibfiçten i̊berbenfen! - Bey ben Crás bigen aber muffen fid sie Ermalynungen zur (B)oulb befonberb anf ben Gerrlicbeu 2fuвgang alles besjentigen Reibenz, welches Ehrifen unter ber 2lufficht uno Regierung ibres guten (sottes begegnet, grumben. Naan

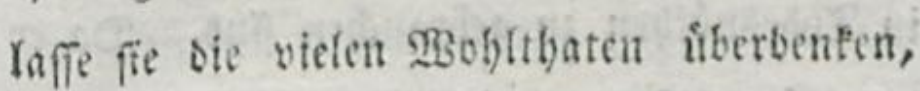
sie fie empfangeu baben! STan erimere fie an bie (Sjebulo ber Jzeiligen, vorzistich)

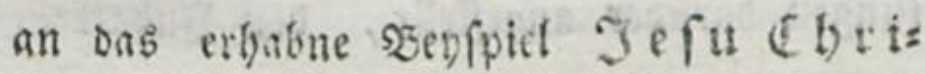
fi. - SRan mus aber and ibre Frcuns De zur (S)oulo ermuntern, ifgre Edwwad)= beiten fanft zu crtragen.

\section{6.}

23cy langwierigen Zirantieiten Sen 2 sefuch balbe Tabte uns vielletht now) !onger fortferen, ift 3 war feine leid)te Eathe; inseß sarf er rod) fols de perfonen nie verlaffen, oem fols d)e Lelende feben oen presiger als ibren wabten freumb an. S.

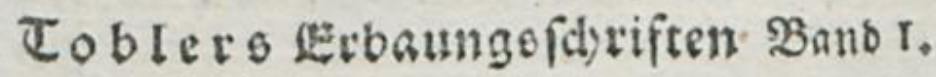

Seis 
Seite 32\%. forg. 2tufmunterung ait perfonen, de bey Jabr uno Eag Erantitić uno mit Gdmersen bebafs tet find. Der \$resiger thut wohl, wemi

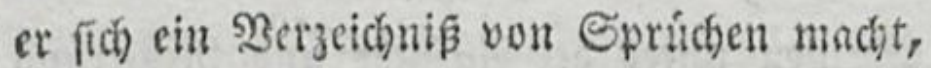
bie beym 2nfang, bey ber Sirntyeit uns in Tobesnithen zu gebraudben fint. Da er bam bey oft zu wieberfyolenben Sifuctien, auffer ben Unterresungen, furze Setrad)= tungen bariber angefellt, ben Silbalt in ein Bebet zufammengefaft, uno mit \$erfen aus \{iebern, bie fich ouf ben Buftano bes Siranfen fojiclen, yerbinbet.

7.

Line Dorbereitung und Etmats nung 3 ur mutbigen Uebernebmung einer former3baften dirurgifenen Opes ration, if ein fo woidstiger freunos fas aftsoienft, saf ein prediger es der v7itle werth adten foll, fich felbf sarzu gefthidtt 3 u madben. - Er muß

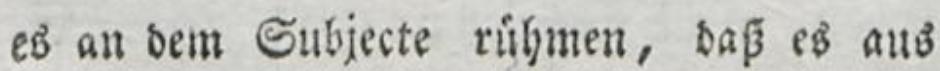
(jess 
(6)borfan gegen Botteg Soronumg, uno aub fouldiger Borforge fir fein Leben, fur

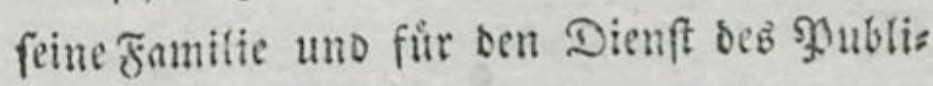
fumb, biefen Selbenmuth Gabe, uno man muf

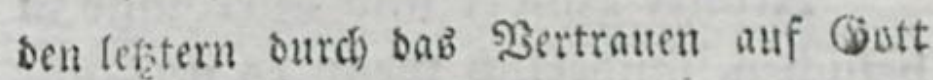

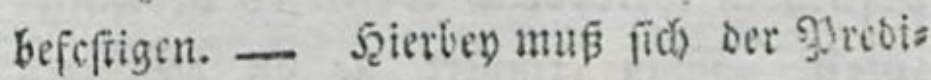
ger felbft yoll guten 9)?utbs zeigen, uno fir ne frente liber bie baldige villige smicst: berfellung febre lebhaft bezcigen, juglcidi aber feine Grunde mit Exempeln yon Sete ben uno Cheiften, welde it ábulidjen llus ftänoen fid) fihr tapfer berwicfen batten, bes leben. Den Seibenden aher am meiffor Durch Das 2 ezeigen Jefu, ber fick in feis nen fósmerzlióen Sciben gánjlich sem \$2Btls Gottes unterworfen, beberzt madien. Lin = ter ber Speration fam ber Bufprud) bes \$rcisigers teinen fonberliçen Einorud auf ben 3yaticnten machen, wohl aber mur ber 23 unos ârate ifger. Der \$ुrebiger fanm fid alfo wo reno bem Edynitte ganz, ober bod wenigs ftens in ein Tetonjimmer entfernen. $S$. Prediger am Karantenbette. Th. I. S. 
I30. S. $680=685$ und vitller 24na leitung S. 183, น. f.

8.

tEvanle Lૈinoer fino allerbings $3^{4}$ befuchen, fonderlich wenn es verIangt witt. Man crinnert fie an oiejenigen auf ibren Buftand fich fajidende febren, bie fie im Eatedibamo geternt baben, wie aud̆ an leidte, won ihnen erlernte Spruide uns 3erfe aแร @iebern, mit befándiger 2lpplicas tion, gibt ifnen ald eine furze 2inneifung, wie fie relbft aus bent Serzen beten uno Sott ifre Noth vortragen follen. - Bey zarten Rinoern, bie noch nid)tz yon (Egris ffenthum gefaßpt baben und gar leines aus fprudb fähig fins, fann man weiter গiäts thutu, alb dafí man fie oer Barmberzigleit (Botteb empfieblt uno bie betrúbten E్ftern tróftet. S. presiger am zirantenbette Th. I. S. I32. S. $690=695$. D. 2100 fenmนำเ 1. c, \$. 129. S. 175 . 
9.

Wirs es verlangt, fo mag des Presiger beym Eterben eines Zirans fen gegenwastig feyn; bod niăt eigents (i⿱亠凶禸 um bes Sterbenben, fonoern vielmebe 3ur um ber febenben willen. So lange ber Sterbende nod Rennjeiden yom ßerfanbe uno Benustfenn von fid gibt, fo betet man für ihn, onj Gjott feinen Tobegfampf verturzen, uno ifm eine felige Aluflofung im frieden verleiken wolle.

Die Finfegnung gefófebt in einem Gerg= liden Dante fur allez, bem Sterbenben in feinem ganzen \&eben, an Seel' uno \&eib er" wiefene Bute, uno bey frommen Ebrio ffell in einer froblichen $\mathfrak{B}$ ezeugung ber guten Joffung bey feinem $2(b$ fodiede.

ఇadobem er verfibiebett, fann, nebft spieberbolung bes bemútbigen Danfeb̉ gegen Gott fúr ben \$erftorbenen, Diefe wichtige 
33egebenbeit auci für sie Hebertebenben wiă,

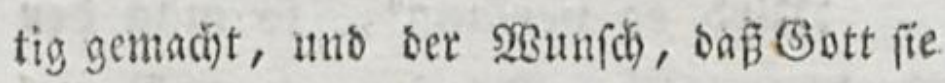
Iflle jult einem feligen ๔terben bereit machen wolle, bengefigt werben. 2fuch sarp man nicht yergiefyen, bie Scinterbliebenen, wo eb nistgig iff, ju triften. S. Preoiger am Lixanfenbette. I\%. I. 6. 123. S. 637" 647. S. I21. S. $047=650$. D. $210=$ fen 23eytrige zut paftoraltbeologie. I, Eap. I.

$$
10 .
$$

Stan fot bemertet, daßs bey Der= forcu, die liber ro Jabr sit find, auf 3lucy Drittbeile oer Ziranrbeiten die Gethefung, uno nut ein Drittlocil ser Tos erfolge. Damit mun ber Jirans Fenbefuch nic)t blop cine Sorbercitung zum Eterben, fonbern auch cine InTeitung uns Ermunterung ju cinem beiligen \&eben wers sen mige; fo mus nach ber senefung beb

Sirans 


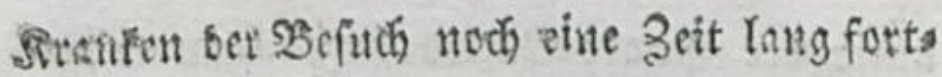
geferst werben. Nan bitte ben Benefenert sie 2lbididt (jottes bey diefer Rrantheit woht zu beberzigen; mit rechtem Ernft woht jut erwigen, wie fein Sd)iffal wirbe gemefert fenn, wenn biefe Rranélyeit die lef̧zte gea wefen wáre, uno was für Entichliepanngen er auf feinem Siranfenbette gefä̈t babe. Man crmalgne ifh, jeben wäbreno feimer

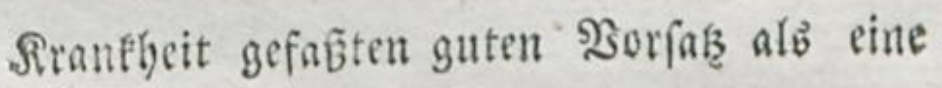
2rt eineb feyerlichen (jelíbseb zu betrad)ten, bey beffer 2(ufric)tigteit eb bauptfichlich auf

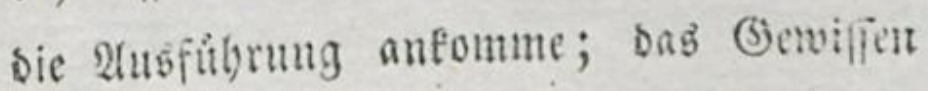

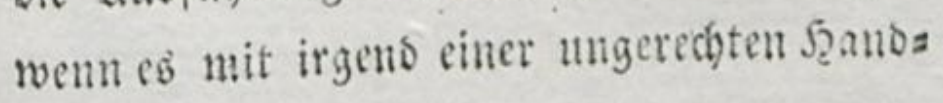
fung befdwert ware, mtunetyr los ju mas chen uns oie 2BBiebererffattung ungeffumt flt bewerffelligen, auch fich gern bor ben Ber: fillyungen zur Sinse, bey feinem nettert Eintritt in sie 28 ett, ourd Sebeth uno

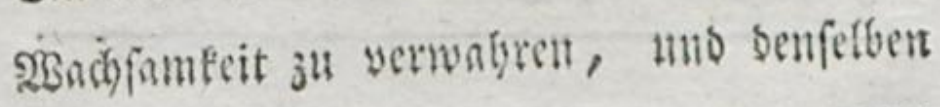
unter 
276

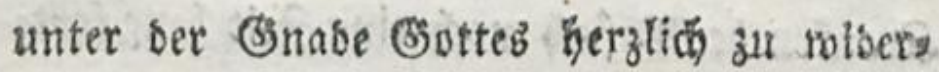

ftehen. S. Presiger an Evatenb. T\%.

I. ๑. I33. D. 2iofenmuluer 1. c.

\$. $I_{33}$.

Enbe bes exfen ふุheils.

fุanกดทer,

georndet bey . T. $\mathfrak{L}$ a mminger. 


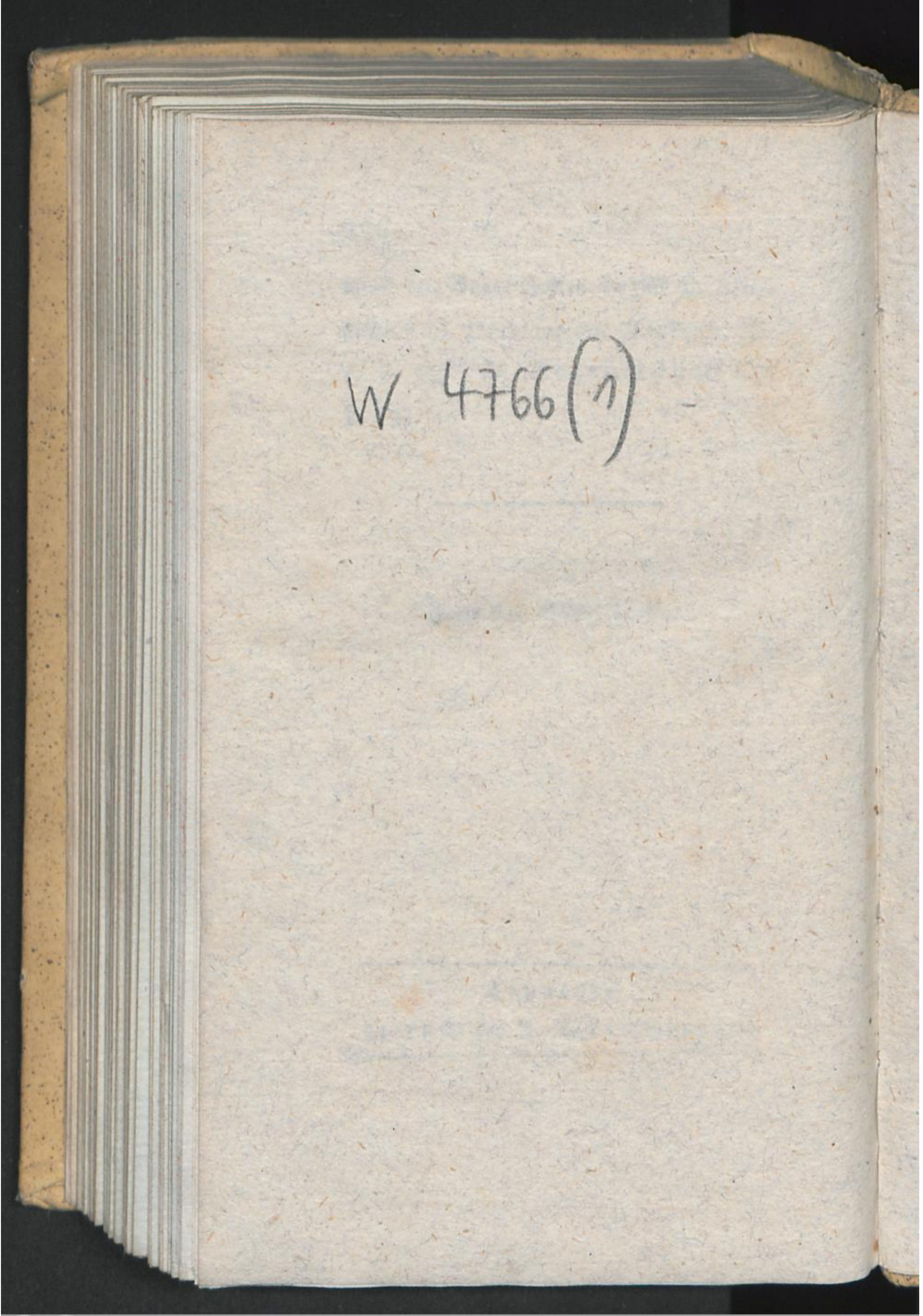




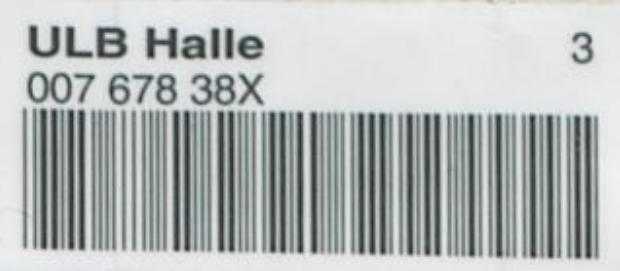

$$
1978
$$




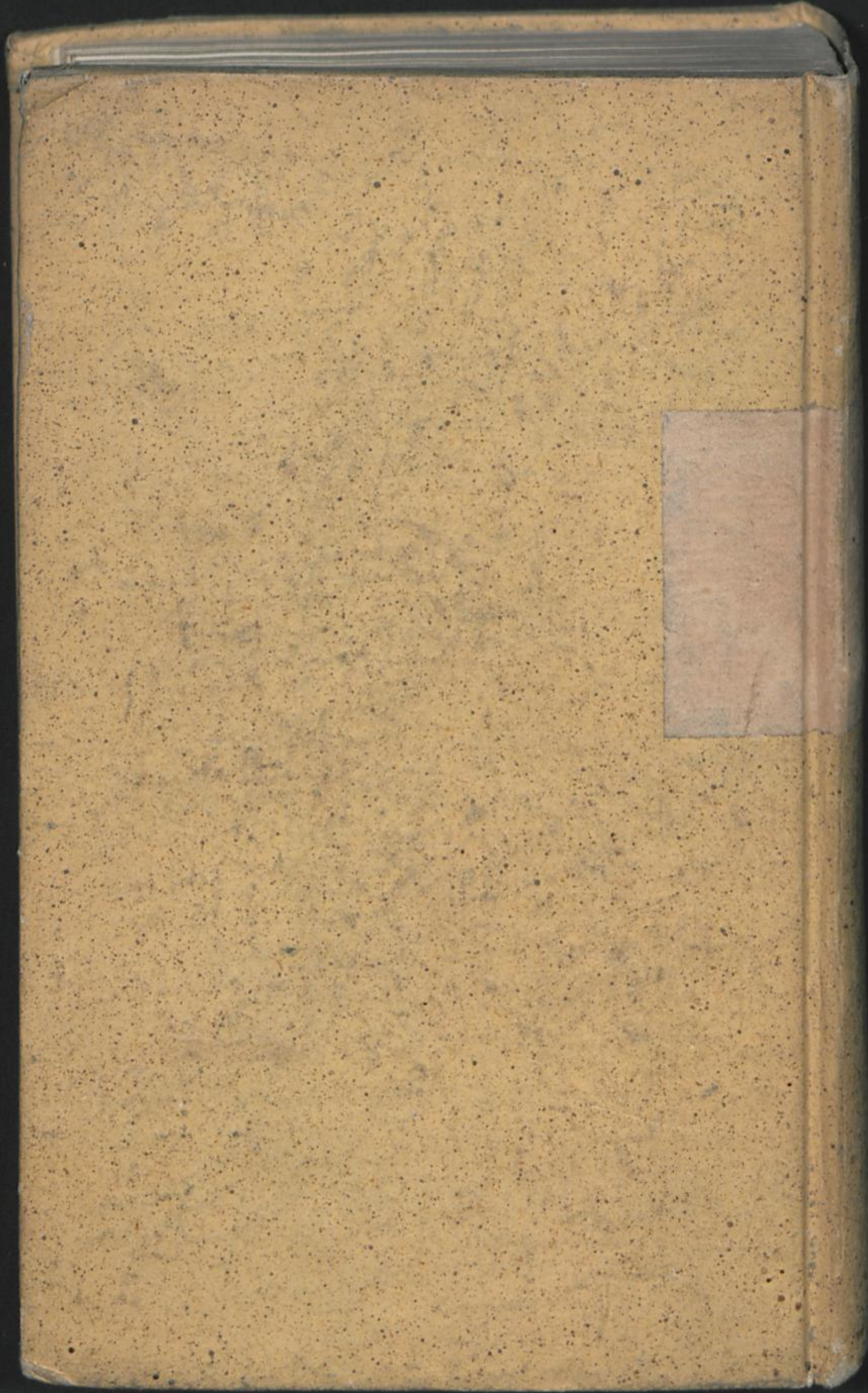

